Bernd Tesch

\title{
Sinnkonstruktion im Fremdsprachenunterricht
}

Rekonstruktive Fremdsprachenforschung mit der Dokumentarischen Methode 


\section{Bernd Tesch}

\section{Sinnkonstruktion im Fremdsprachenunterricht}

Das Buch führt in die rekonstruktive Fremdsprachenforschung mit Hilfe der Dokumentarischen Methode ein. Dabei betrachtet der Autor den Fremdsprachenunterricht praxeologisch, d.h. nicht wie er idealerweise sein sollte oder könnte, sondern im Hinblick darauf, welche Sinnkonstruktionen in der Alltagspraxis stattfinden. Es werden dazu Unterrichtsbeobachtungen in natürlicher Lernumgebung, aber auch Gruppengespräche mit Lehrenden und Lernenden sowie Einzelinterviews genutzt. Im Ergebnis stellt sich der Fremdsprachenunterricht als Konstruktionsprozess auf verschiedenen Ebenen dar.
Der Autor illustriert das Verfahren an konkreten Beispielen aus der, Fallwerkstatt', so dass das Buch auch als methodischmethodologisches Lehr- und Arbeitsbuch verwendet werden kann.

\section{Der Autor}

Bernd Tesch ist Professor für Fremdsprachenlehr- und -lernforschung mit dem Schwerpunkt Didaktik des Französischen und Spanischen an der Universität Kassel. Er arbeitete an Schulen im In- und Ausland und war am Berliner Institut zur Qualitätsentwicklung im Bildungswesen tätig. 
Sinnkonstruktion im Fremdsprachenunterrich1 
Bernd Tesch - 978-3-631-69542-5

Downloaded from PubFactory at 01/11/2019 10:14:38AM

via free access 
Bernd Tesch

\section{Sinnkonstruktion im Fremdsprachenunterricht}

Rekonstruktive Fremdsprachenforschung mit der Dokumentarischen Methode

\section{PETER LANG}




\title{
Bibliografische Information der Deutschen Nationalbibliothek
}

Die Deutsche Nationalbibliothek verzeichnet diese Publikation in der Deutschen Nationalbibliografie; detaillierte bibliografische Daten sind im Internet über http://dnb.d-nb.de abrufbar.

\author{
ISBN 978-3-631-67556-4 (Print) \\ E-ISBN 978-3-653-07083-5 (E-PDF) \\ E-ISBN 978-3-631-69542-5 (EPUB) \\ E-ISBN 978-3-631-69543-2 (MOBI) \\ DOI 10.3726/978-3-653-07083-5
}

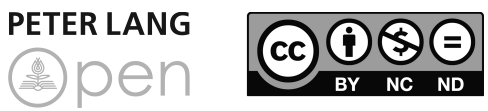

Open Access: Dieses Werk ist lizensiert unter der Creative Commons Lizenz Namensnennung - Nicht kommerziell - Keine Bearbeitungen 4.0 International (CC BY-NC-ND 4.0). Den vollständigen Lizenztext finden Sie unter: https://creativecommons.org/licenses/by-nc-nd/4.0/deed.de

Diese Publikation wurde begutachtet.

\author{
(C) Bernd Tesch, 2018 \\ Peter Lang GmbH \\ Internationaler Verlag der Wissenschaften \\ Frankfurt am Main 2016 \\ Peter Lang - Frankfurt am Main · Bern · Bruxelles · \\ New York · Oxford · Warszawa · Wien \\ www.peterlang.com
}


Diese Schrift ist meinen Studierenden gewidmet. 
Bernd Tesch - 978-3-631-69542-5

Downloaded from PubFactory at 01/11/2019 10:14:38AM

via free access 


\section{Vorwort}

Diese Schrift ist als Einführung in die rekonstruktive Fremdsprachenforschung mit der Dokumentarischen Methode konzipiert. Sie entstand in den Jahren 2015-2016 auf Grundlage der Auswertung eines kontinuierlich durchgeführten Forschungsseminars zur rekonstruktiven Fremdsprachenforschung an der Universität Kassel und unter Rückbezug auf frühere Forschungen mit der Dokumentarischen Methode im Fremdsprachenbereich (Bonnet 2004, 2009; Tesch 2010). Die Weiterentwicklung meiner 2010 veröffentlichten Arbeit umfasst insbesondere eine intensive Reflexion der Rolle der Lernersprachen und der Sprachbewusstheit im Rahmen der unterrichtlichen Interaktion sowie die Einbeziehung des narrativen Interviews (Nohl 2005, 2012). Auch die dokumentarische Analyse von Texten im Hinblick auf ihre Sinngehalte wurde berücksichtigt, um die Anwendungsmöglichkeiten der Dokumentarischen Methode im Bereich der Fremdsprachenforschung möglichst vollständig abzubilden.

Die Besonderheit des Fremdsprachenunterrichts gegenüber muttersprachlichem Unterricht besteht in der partiellen oder vollständigen Unterrichtskommunikation in einer anderen Sprache. Dieser Umstand beeinflusst die Diskursentwicklung, die Typenbildung sowie die für den Fremdsprachenunterricht charakteristische Relation von Sozialität und Individualität in entscheidendem Maße. Er macht folgerichtig die Einbeziehung des lernersprachlichen Sinns und der Sprachbewusstheit in die Analysen zwingend erforderlich.

Das narrative Interview kann eigenständig für die Forschung zu Sprachenund Berufsbiografien und zu professionellen Haltungen und Einstellungen von Fremdsprachenlehrerinnen- und -lehrern eingesetzt werden und wertvolle Einsichten in biografische Formungen liefern. Es kann in ähnlicher Weise auch für die Lernendenforschung genutzt werden. Und schließlich kann es - auch in seiner verkürzten Variante - als Triangulationsinstrument für die Unterrichtsforschung eingesetzt werden, um begleitende Informationen zu bestimmten Berufs- oder Lernabschnitten oder zu Klassensituationen punktuell beizusteuern.

Die Verständigung durch Texte schließlich und die Analyse dieser Verständigung bildet traditionell den Kernbestand des Fremdsprachenunterrichts und ist als solcher bereits sehr umfassend in der fachdidaktischen Forschung repräsentiert. Allerdings kann auch hier die Dokumentarische Methode nutzbringend vor allem im Hinblick auf die Bildanalyse sowie die Analyse mehrmodaler Texte (Film, Comic, etc.) angewendet werden. 
Die Übereinstimmungen der rekonstruktiven Fremdsprachenforschung mit der rekonstruktiven Sozialforschung (Bohnsack 2014), aus der sie hervorging, liegen auf der Hand. Ebenso evident ist jedoch die Notwendigkeit, die besonderen fremdsprachenunterrichtlichen Transformationen zu beschreiben, die dazu führten, die Dokumentarische Methode für die rekonstruktive Fremdsprachenforschung weiterzuentwickeln und ihr allgemeines heuristisches Potential auszuschöpfen. Diesem Ziel ist die vorliegende Schrift verpflichtet.

Die gemeinsame Abbildung der drei genannten und sehr unterschiedlichen Foki - Unterrichtsforschung, narratives Interview und Text- und Bildanalyse in einem einzigen und zudem sehr komprimierten Band stellt zweifellos ein Wagnis dar, zumal das hier vorgestellte Verfahren der Text- und Bildanalyse auch in der Literatur- und in der Medienwissenschaft gängig ist. Ich bin allerdings der festen Überzeugung, dass die gemeinsame Klammer der Differenzierung und Rekonstruktion von Sinnebenen sowie die Illustration der ihr inhärenten Arbeitsschritte das Vorhaben rechtfertigt.

Um diese gemeinsame Klammer der verschiedenen Foki nachvollziehen zu können, empfiehlt es sich, den Band durchgehend zu lesen. Die vier Hauptkapitel bauen vom Allgemeinen zum Spezifischen hin aufeinander auf. Das erste Kapitel bildet eine allgemeine Einführung in die rekonstruktive Fremdsprachenforschung mit der Dokumentarischen Methode. Im zweiten Kapitel werden das elementare Begriffsinventar sowie die zentralen Sinnebenen, die den Gegenstand der rekonstruktiven Fremdsprachenforschung bilden, eingeführt und erläutert. Im dritten Kapitel werden die Arbeitschritte der Dokumentarischen Methode vorgestellt, und im vierten Kapitel werden Einblicke in die rekonstruktive Methodik an Hand konkreter Beispiele vermittelt.

Ich danke allen Studierenden und Lehrkräften, die an seiner Entstehung mitgewirkt haben sowie Linda Pelchat für das Lektorat. Mein besonderer Dank gilt Gianna Ina Winkels sowie den Interviewpartnerinnen „Anne“ und „Bianca“.

Kassel, im Juni 2016

Bernd Tesch 


\section{Inhaltsverzeichnis}

1 Einleitung: Der rekonstruktive Zugang zum Fremdsprachenlehren und -lernen

2 Sinnkonstruktion im Fremdsprachenunterricht ...........................25

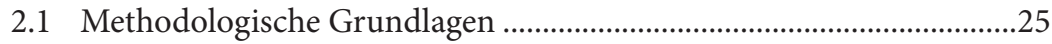

2.1.1 Methodisch kontrolliertes Fremdverstehen ................................. 25

2.1.2 Diskursentwicklung im Fremdsprachenunterricht ...................... 27

2.1.3 Die Indexikalität der Verständigung ............................................... 32

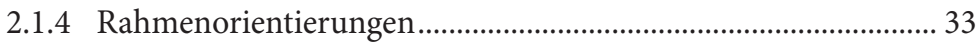

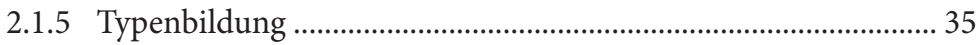

2.1.6 Lernersprache ................................................................................. 38

2.1.7 Lerngruppenmilieus und konjunktive Erfahrungsräume.......... 43

2.1.8 Ein sozial-interaktionales Lehr-Lernmodell des Fremdsprachenunterrichts......................................................... 47

2.2 Verständigung durch Texte......................................................................50

2.2.1 Verständigung durch literarische Texte .......................................... 51

2.2.2 Verständigung durch Bildtexte …………………………................ 55

2.3 Verständigung über Texte.........................................................................61

2.3.1 Bild-Text-Aufgabe........................................................................... 62

2.3.2 Aufgabe Personenbeschreibung..................................................... 66

2.4 Verständigung über erlebten Fremdsprachenunterricht, über das Lehren und über das Lernen von Sprachen

3 Arbeitsschritte der Dokumentarischen Methode..........................79

3.1 Gruppendiskussion und Unterrichtsgespräch ......................................79

3.1.1 Teilnehmende Beobachtung, Audioaufzeichnung

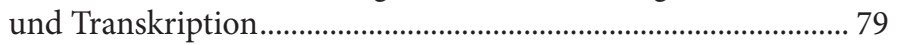

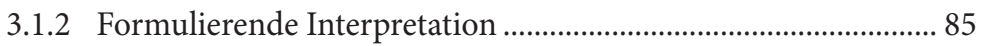


3.1.3 Reflektierende Interpretation ........................................................... 88

3.1.4 Fallbeschreibung ....................................................................... 92

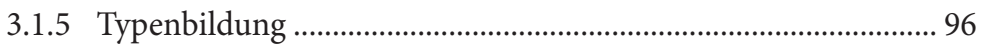

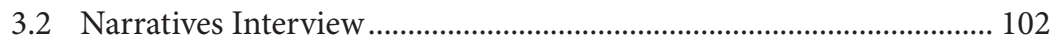

3.2.1 Formale Interviewanalyse.......................................................... 102

3.2.2 Inhaltliche Interviewanalyse......................................................... 104

3.2.3 Fallbeschreibung und Fallvergleich............................................. 105

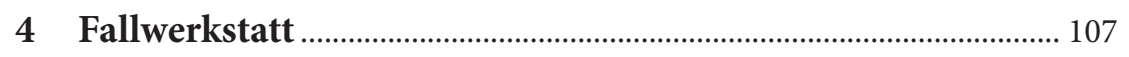

4.1 Der Comic Ne me quitte pas im Spanischunterricht.......................... 107

4.1.1 Forschungsfragen und Forschungsrahmen................................ 107

4.1.2 Jordi Lafebre, Ne me quitte pas: Analyse des Textsinns............. 109

4.1.3 Ne me quitte pas: Analyse des Aufgabensinns ............................. 113

4.1.4 Vom Was zum Wie: Wechsel der Analyseeinstellung................. 120

4.1.5 Fallvergleich und Typenbildung .................................................... 131

4.2 Die Verständigung über das Lehren von Sprachen.

Narrative Interviews mit Bianca und Anne.......................................... 136

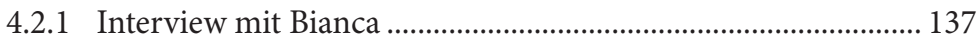

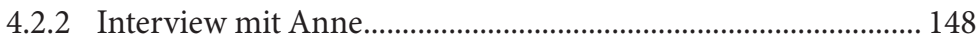

4.3.3 Fallvergleich narrativer Interviews ............................................. 155

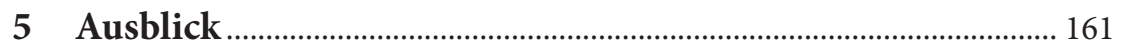

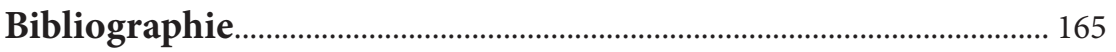

Abbildungs- und Tabellenverzeichnis ................................................... 175

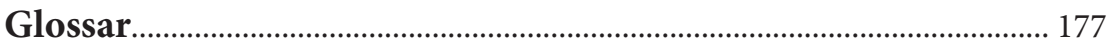

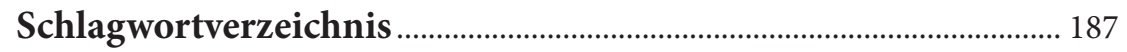




\section{Einleitung: Der rekonstruktive Zugang zum Fremdsprachenlehren und -lernen}

\section{Auf der Suche nach einem methodologischen Referenzrahmen}

Unter Mark Twains recht bekannten und in der Regel wenig schmeichelhaften Zitaten über das Erlernen der deutschen Sprache fällt eines in Auge, in dem der Autor das Erlernen des Deutschen mit dem Erlernen des Englischen und dem Erlernen des Französischen vergleicht:

My philological studies have satisfied me that a gifted person ought to learn English (barring spelling and pronouncing) in thirty hours, French in thirty days, and German in thirty years. It seems manifest, then, that the latter tongue ought to be trimmed down and repaired. If it is to remain as it is, it ought to be gently and reverently set aside among the dead languages, for only the dead have time to learn it.

(Appendix D von A Tramp Abroad, "That Awful German Language”)

Meine philologischen Studien haben mich darin bestätigt, dass eine begabte Person Englisch (mit Ausnahme von Orthografie und Aussprache) in dreißig Stunden, Französisch in dreißig Tagen und Deutsch in dreißig Jahren lernen sollte. Es scheint somit auf der Hand zu liegen, dass die letztere Sprache heruntergestutzt und repariert werden sollte. Sollte sie allerdings so bleiben, wie sie ist, so sollte sie sanft und ehrfürchtig zu den toten Sprachen beiseite gelegt werden, da nur die Toten die Zeit haben, sie zu lernen. (Übers. BT)

Ganz offensichtlich waren Mark Twains Lernerlebnisse mit dem Deutschen negativ besetzt. Man könnte nun - Twain folgend - die Ursachen dafür vorwiegend in der deutschen Sprache suchen: zu schwer (mühsam, kompliziert, ...) zum Lernen. Man könnte Twain mit einem Augenzwinkern freilich auch als einen Probanden für rekonstruktive Fremdsprachenforschung heranziehen, spiegeln seine Betrachtungen doch, bei aller beabsichtigten Ironie, sozial geteiltes Wissen bzw. sozial geteilte Muster, vor allem das Muster der schweren deutschen Sprache im Vergleich zu anderen Sprachen. Twain verarbeitete seine Frustration beim Fremdsprachenlernen (das „Was“ seiner Aussage) mit dem Mittel der Ironie (das „Wie“). Diese könnte den tieferen Ursprung seiner Frustration verbergen, nämlich das Bedürfnis nach Lernerfolg und Effizienz. Beides blieb ihm beim Versuch, die deutsche Sprache zu lernen, möglicherweise versagt.

Natürlich könnte man die Geschichte einer Begegnung mit der deutschen Sprache auch ganz anders erzählen. Worauf ich mit diesem Beispiel abziele, ist Folgendes: Das Fremdsprachenlernen entspricht genauso wie jedes Lernen einer 
Sinnkonstruktion ${ }^{1}$. Diese ist individuell und kollektiv zugleich. Kollektiv ist sie insofern, als jeder Mensch bestimmte Lebensumstände und somit Erfahrungen, Hoffnungen und Erwartungen mit anderen Menschen teilt. Individuell ist sie, insofern der Einzelne seine Erfahrungen, Hoffnungen und Erwartungen in individuelle Lernentscheidungen gießt. Gelingendes Fremdsprachenlernen folgt also auch einer gelingenden Sinnkonstitution, z.B. in Form einer individuellen Identifikation mit Texten und kulturellen Erwartungen, mit Lehrpersonen und Lernarrangements, mit persönlichen Kompetenz- oder Effizienzerwartungen. Diese sinnstiftenden Identifikationen können freilich auch ausbleiben und dadurch das Erlernen einer Sprache verhindern. Lernen als Sinnkonstruktion kann dieser Schrift als anthropologische Grundannahme vorangestellt werden.

Nach wie vor fällt es schwer, die außerordentliche Komplexität des Fremdsprachenlehrens und -lernens und insbesondere des fremdsprachlichen Unterrichtsalltags empirisch zu erforschen. Neben einigen forschungspraktischen Herausforderungen - erforderlich sind die Bereitschaft von Lehrkräften und Lernenden ${ }^{2}$, u. U. auch die Genehmigung der Eltern, der Schulleitung, der Schulkonferenz sowie der Schulbehörden - stellt sich elementar die Frage nach einem geeigneten methodologischen Referenzrahmen. Bei standardisierten bzw. quantifizierenden Verfahren besteht das Problem, dass es bei messtechnisch hohen Standards (z.B. quasi-experimentelle Studien mit Kontrollgruppen) oft nicht möglich ist, die natürliche Lernumgebung und die Komplexität der Faktoren sowie die Dynamik der Unterrichtsprozesse adäquat zu erfassen. Um nur ein Beispiel zu nennen: Wie könnte mit einem standardisierten Verfahren die ganze Komplexität des literarischen Textverstehens bei einer Gruppenarbeit adäquat erfasst werden, wo es offensichtlich zu einer Vielzahl individueller und unvorhersehbarer Aushandlungsprozesse kommt?

1 In dieser Schrift ist in der Regel von Sinnkonstruktion die Rede, nur gelegentlich verwende ich die Bezeichnung Sinnkonstitution. Der Begriff der Sinnkonstitution spielt in der Phänomenologie von Alfred Schütz eine zentrale Rolle (Schütz 1974, s. Thomas 1984) und weist mithin eine semantische und konzeptuelle Nähe zum Begriff der Sinnkonstruktion in der Wissenssoziologie Karl Mannheims (1980) auf. Die hier bevorzugte Verwendung von Sinnkonstruktion lehnt sich jedoch stärker an die Wissenssoziologie als an die Phänomenologie an, insofern nämlich die sozialen Prozesse des Hervorbringens von Sinn (Konstruktion) enger im Zentrum der Betrachtung stehen als die Beschaffenheit der Phänomene (Konstitution), die aus diesen Prozessen resultieren.

2 In weiten Teilen der Schrift wird darauf geachtet, allgemeine bzw. generische Geschlechterbezeichnungen zu verwenden. An Stellen, wo dies zu Lasten der Lesbarkeit gehen würde, wird die männliche Form im generischen Sinne verwendet. 
Diese und ähnliche Überlegungen veranlassten mich bereits in den Jahren 2006 bis 2009 bei der Arbeit an einer Studie zum Unterricht mit bildungsstandardbasierten Lernaufgaben, mich für offenere methodische Zugänge zu interessieren. Angeregt durch die Begegnung mit Barbara Asbrand, die bereits seit längerem mit der Dokumentarischen Methode arbeitete und zum damaligen Zeitpunkt das globale Lernen an schulischen und außerschulischen Lernorten (Asbrand 2009) erforschte, entschloss ich mich, mit dieser Methode weiterzuarbeiten und entdeckte nach und nach ihr Potential auch für die Fremdsprachenforschung. Von 2006 bis 2007 hatte ich die Gelegenheit, an der Forschungswerkstatt Ralf Bohnsacks, des Mitbegünders der Dokumentarischen Methode, teilzunehmen. Mir wurde bald klar, dass rekonstruktive Verfahren den von mir gesuchten Referenzrahmen für die Verständigungsprozesse im Kontext des Fremdsprachenlehrens und -lernens darstellen könnten. Und die bekannten spezifischen Verfahren der Konversationsanalyse, Narrationsanalyse, objektiven Hermeneutik sowie Dokumentarischen Methode wiesen offensichtlich große Übereinstimmungen in den methodologischen Kernfragen des interpretativen Verstehens von Verständigung auf. Mir wurde überdies deutlich, dass mein Interesse ein fundamental praxeologisches war: Worin besteht eigentlich die Praxis des Fremdsprachenlehrens und -lernens? Welche Sinnkonstruktionen finden in der Praxis des Fremdsprachenunterrichts statt? Welche Sinnebenen konstituieren eine Lernsituation in der Praxis (z.B. Bewältigung einer gestellten Aufgabe, individuelles Leseinteresse, soziale Erwartungen des Elternhauses, Position in der Hierarchie der Peers)? Mein Forscherinteresse lag bald nicht mehr bei der Suche nach gelingender oder nicht gelingender Aufgabenbearbeitung, nach guten oder weniger guten ${ }^{3}$ Lernaufgaben, sondern bei ganz anderen Fragestellungen, die sich nämlich auf die Verstehens- und Verständigungspraxen in einer Fremdsprache und im Fremdsprachenunterricht sowie auf Praxen der Verständigung über das Lehren und Lernen von Fremdsprachen richteten.

3 Der im Zuge der Einführung der Kompetenzorientierung aufkommende Diskurs über "gute Aufgaben“" (Ulm et al. 2008; Bremerich-Vos et al. 2008) erweckte in der Anfangsphase den Eindruck, eine Weiterentwicklung der „Aufgabenkultur“ im Sinne kompetenzorientierter Lern- und Prüfungsaufgaben habe per se bereits das Potential, den Unterricht selbst positiv zu verändern. Diese Vorstellung lenkt m. E. das Augenmerk zu sehr auf den Input und verkennt die Bedeutung der Prozessfaktoren für gelingenden Unterricht. Zu den Prozessfaktoren gehören m. E. vor allem die typischen Lehr- und Lernhandlungskonzepte, das heißt, das implizite und handlungsleitende Wissen der Lehrenden und der Lernenden (vgl. Tesch 2010). 


\section{Praxeologische Fremdsprachenforschung}

Dokumentarische Fremdsprachenforschung (z.B. Bonnet 2004, 2009; Bracker 2015; Tesch 2009; fächerübergreifend: Ballis et al. 2014) ist empirisch-praxeologische Forschung. Sie untersucht nicht, was alles beim Fremdsprachenlehren und -lernen stattfinden sollte oder könnte, also die Welt des Konzeptionellen, Normativen oder Potentiellen, und auch nicht die messbaren Resultate des Fremdsprachenlehrens und -lernens, sondern sie richtet den Blick auf die Praxis, auf das, was in der Praxis des Fremdsprachenlehrens- und -lernens geschieht und vor allem darauf, wie es geschieht. Sie nimmt gleichzeitig eine veränderte, nämlich an sozialen Verständigungsprozessen orientierte Perspektive bzw. Analyseeinstellung zum Unterricht ein und hat ein eigenes Erkenntnisinteresse. Somit wiederum stellt sie auch eine ganz natürliche und zugleich notwendige Ergänzung zur fachdidaktischen Aufgabenforschung dar (für die Fremdsprachen z.B. Ellis 2003; Finkbeiner 2008, 2013; Müller-Hartmann \& Schocker-v.-Dithfurth 2011; Tesch et al. 2016), die z. T. sehr detailliert Aufgabenkonstituenten und Faktoren der Aufgabenbearbeitung beschreibt und analysiert. Diese Verortung der rekonstruktiven Fremdsprachenforschung ist an das Angebot-Nutzungs-Modell Helmkes (Helmke 2002) anschlussfähig: Aufgaben können als Teil des unterrichtlichen Angebots, ihre praktische Umsetzung als Nutzung betrachtet werden. Rekonstruktive Fremdsprachenforschung ist also - soweit sie das Unterrichtsgeschehen selbst untersucht - auch Aufgabennutzungs- bzw. Aufgabenbearbeitungsforschung. Diese wiederum ist nicht mit der Aufgabenwirkungsforschung - etwa in Form standardisierter Messungen zu Kompetenzständen - zu verwechseln. Und nicht zuletzt sind sowohl die Aufgabenforschung als auch die Aufgabenbearbeitungsforschung einzubetten in eine umfassende Theorie des fremdsprachlichen Klassenzimmers, die allerdings erst in Konturen sichtbar ist (vgl. Breen 1985; Esteve 2010; Legutke 2006) und die die Komponenten Lehrende, Lernende, Themen, Texte, Medien, Interaktion und Diskurse umfasst (s. Kap. 5).

Darüberhinaus lassen sich aber auch die formale Gestaltung fremdsprachiger Texte (im weiteren Sinne Schrifttexte, Bilder, Filme etc.) und deren Wirkung auf den Rezipienten empirisch-rekonstruktiv erforschen. Als Sprach- und Handlungspraxis untersucht die rekonstruktive Fremdsprachenforschung somit die Verständigung durch (fremdsprachige) Texte (s. Kap. 2.2, 3.1, 4.1), die Verständigung durch Aufgaben über Texte (s. Kap. 2.3, 3.1, 4.1) und schließlich auch die Verständigung über den Fremdsprachenunterricht sowie das Lehren und Lernen von Sprachen (s. Kap. 2.4, 3.2, 4.2). Für die Untersuchung der Verständigung über das Lehren und Lernen von Sprachen lässt sich die Dokumentarische Methode überdies mit der Technik des narrativen Interviews verbinden (s. Kap. 3.2, 4.2). 
Die Praxis des Fremdsprachenunterrichts wird von mir als soziale und individuelle Sinnkonstruktion verstanden, eingebunden in kommunikatives Handeln in Gruppen oder im Klassenplenum, aber auch in Einzel- bzw. Stillarbeit. ${ }^{4}$ Rekonstruktive Fremdsprachenforschung, die diese Praxis untersucht, kann nach Bohnsack (2014: 25) als „Konstruktion zweiten Grades“ bezeichnet werden oder als die Re-Konstruktion einer (sozial) konstruierten Praxis.

Aber auch die Forschungspraxis selbst wird wieder Gegenstand von Rekonstruktion. So leitet Bohnsack (2014: 19-21) aus der Tatsache, dass sie denselben Produktionsgesetzmäßigkeiten unterliegt wie die Praxis, die sie erforscht, Validitätsansprüche der rekonstruktiven Sozialforschung $\mathrm{ab}^{5}$ (s. Kap. 2.1). Unter der Formel „Rekonstruktion der Rekonstruktion“ (ibd. 27) versteht er die Untersuchung der rekonstruktiven Forschungspraxis selbst bzw. der Standortgebundenheit des Forschenden.

Mit welchen Daten arbeitet rekonstruktive Fremdsprachenforschung? Es handelt sich meist um gesprochene oder geschriebene fremdsprachliche (die Fremdsprachen betreffende) oder fremdsprachige (in der fremden Sprache verfasste) Texte aller Art, ferner Bildtexte oder mehrmodale Texte. Es handelt sich häufig um aufgezeichnete Gespräche über Fremdsprache(n) und über das Lernen von Fremdsprachen, Gespräche über das Lehren von Fremdsprachen und sogar Gespräche über das Lernen vom Lehren von Fremdsprachen. Kurzum, alle erdenklichen Texte, die im direkten oder indirekten Zusammenhang mit dem Lernen und Lehren von Fremdsprachen generiert worden sind, können Gegenstand rekonstruktiver Fremdsprachenforschung sein. Die Gesprächstexte sind real: Sie werden ,im Feld produziert, und müssen daher zur forschenden Verarbeitung gesichert (z.B. audiografiert) und dann ausgewertet werden. In allen

4 Bei der Sichtung von Unterrichtsvideos und von Unterrichtstranskripten in der Literatur fällt ein schwerwiegendes Desiderat ins Auge. Entweder findet man kurze Episoden über wenige Rede-Antwort-Sequenzen hinweg (sog. turns), häufig von Forschenden mit einem applied-linguistics-Hintergrund, oder man findet Lehrvideos einer ganzen Unterrichtsstunde, häufig aus der Bildungsforschung und mit einem Fokus auf Lehrerinnenund Lehrerforschung oder Klassenatmosphäre. Diese sind jedoch mehr oder weniger inszeniert. Der ,natürliche` Unterricht mit allen Abbrüchen und Unterbrechungen, mit Flüstergesprächen und Nebenhandlungen ist eine Leerstelle der Forschung.

5 Bohnsack erläutert ausführlich (2014: 15 ff.) die Unterschiede und Gemeinsamkeiten hypothesenprüfender und rekonstruktiver Verfahren. M. E. genießt der erkenntnistheoretische bzw. methodologische Vergleich von Forschungsparadigmen bisher in der Fremdsprachenforschung noch eine zu geringe Beachtung, was dazu führt, dass die zu beobachtende Scheu bzw. Widerstände gegenüber anderen Paradigmen als dem schwerpunktmäßig eigenen schwer zu überwinden sind. Siehe Kap. 3.1., Fußnote 4. 
Fällen mündlicher Textdaten beginnt die Auswertung mit der Transkription. In zahlreichen Fällen handelt es sich zudem um Daten, die ,im Feld' des Fremdsprachenunterrichts und damit in einer natürlichen oder relativ natürlichen Lernumgebung erhoben wurden und nicht um Daten, die aus Experimenten oder experimentellen Anordnungen hervorgingen.

\section{Konstruktionsprozesse}

Was macht das Besondere der Dokumentarischen Methode aus, was unterscheidet sie von anderen nicht standardisierten Untersuchungsmethoden, die ebenfalls Unterrichtsdaten erheben? Eine der grundlegenden Erkenntnisse der Wissenssoziologie Karl Mannheims (1922, 1980), auf die sich die dokumentarisch Forschenden stets beziehen, aber auch des auf George Herbert Mead (1934) zurückgehenden symbolischen Interaktionismus sowie der phänomenologischen Soziologie von Alfred Schütz (1974) liegt darin, dass Verständigung vor dem Hintergrund sozial geteilter bzw. „konjunktiver Erfahrungsräume“ (Mannheim 1980: 272) - kollektiv erzeugt wird. Individuen erzeugen, in der Terminologie der Dokumentarischen Methode, sozial geteilte „Orientierungsmuster" (Bohnsack 2014: 39). Diese Orientierungsmuster sind meist unbewusst und werden als vortheoretisches bzw. in der Terminologie Karl Mannheims als ,,atheoretisches Wissen“ (Mannheim 1980: 73 ff.) bezeichnet. So äußert eine Gruppe im Rahmen eines Gesprächs oder ein Einzelner im Rahmen eines Interviews unbewusst Sinngehalte, die notiert und anschließend rekonstruiert bzw. gedeutet werden können. „Atheoretisches“ Wissen der Beforschten wird auf diese Weise zu theoretischem Wissen der Forschenden.

Die Verständigung innerhalb einer Gruppe impliziert jedoch nicht, dass die rekonstruierten Orientierungen auch im selben Rahmen liegen müssen, was heißt: Sie können kongruent ebenso wie inkongruent sein. Przyborski (2004) unterschied im Bereich kongruenter Rahmungen drei „inkludierende Modi“ und auf der Seite der inkongruenten Rahmungen zwei „exkludierende Modi“ (s. Kap. 3.1.3). In letzterem Falle redet man sozusagen ,aneinander vorbei“.

Rekonstruktive Sozialforschung mit der Dokumentarischen Methode untersucht mithin bestimmte soziale Erfahrungs- und Sinnkonstruktionen, indem sie die Modi ihrer Erzeugung rekonstruiert. Auf diese Weise entsteht ein Bild der habitualisierten Vorstellungen und Orientierungen in einer Gruppe. Diese entsprechen nicht einer objektiven Realität, sondern sie sind vielmehr der subjektiven Realität der sie erzeugenden Personen verhaftet. Diese subjektive Realität ist allerdings handlungsleitend, sie bestimmt das Denken und Handeln der Mitglieder der untersuchten Gruppe in Bezug auf bestimmte Themen. 
Auch eine schulische Lerngruppe und Klasse oder auch ein Universitätsseminar kann als eine Gruppe oder ein Kollektiv betrachtet werden, das einen „konjunktiven Erfahrungsraum“ (Mannheim 1980: 272) und somit sozial geteilte Erfahrungen und Orientierungen besitzt. Im Unterrichtsgespräch oder in einer Gruppenarbeit kommen diese Erfahrungen und Orientierungen zum Vorschein. Der Begriff des „Konjunktiven“, der im Übrigen synonym zu „gemeinsam“ bzw. „sozial geteilt“ gebraucht werden kann, steht metaphorisch für den Vollzug einer kollektiven Praxis.

Die Dimension des gemeinsamen, kollektiven Generierens von Sprache und Bedeutung wurde bisher auch in der Fremdsprachenforschung im Rahmen einer soziokulturellen Theorie des Lernens sowie in der Language AwarenessForschung untersucht (z. B. Allwright 1984; Batstone \& Philp 2013; Breen 1985; James \& Garrett 1991, Lantolf \& Poehner 2008, Van Lier 1984 und 2004). Dabei beziehen sich einige Autoren auf die Forschungen des russischen Psychologen Vygotsky (1886-1934). Dieser hatte in der Auseinandersetzung mit Piaget das Verhältnis von Sprachentwicklung und Denken untersucht und auch die Beziehung zwischen muttersprachlicher und fremdsprachlicher Entwicklung mit einbezogen. Dabei war er zu dem Schluss gekommen, dass der Fremdsprachenerwerb nicht etwa eine Wiederholung des Mutterspracherwerbs darstellt, wie es Piaget vermutete, sondern ein Konstruktionsprozess ganz eigener, individueller Art ist (s. Vygotsky 1971). In diesen Konstruktionsprozess fließen sämtliche zum Zeitpunkt X vorhandenen Wissensbestände ein. Vygotsky sprach in diesem Zusammenhang von der individuellen „Zone der naheliegenden Entwicklung" (ibd.: 1971: 236-245). Da jedoch beim Fremdsprachen- und beim Tertiärsprachenerwerb bereits sprachliches Wissen aus der Muttersprache, ggf. einer Zweitsprache und einer oder mehrerer vorgelernter Fremdsprachen vorhanden ist, lässt sich über die naheliegende Entwicklungszone hinaus weitergehend auf eine „potenzielle Entwicklungszone“ (ibd.) schließen, die ebenfalls durch die Fremdsprachenforschung rekonstruiert werden kann. Aktuelle, naheliegende und potenzielle Entwicklungszonen sind ggf. anschlussfähig an Niveaus des Gemeinsamen europäischen Referenzrahmens für Sprachen (Europarat 2001). Die Bandbreite soziokultureller Forschungen zum Fremdsprachenlernen reicht somit von der Ebene der Lernersprache (Interlanguage, Selinker 1971), die performatorisch als individuell beschrieben, genetisch jedoch als kollektive Konstruktion betrachtet werden kann, bis hin zu komplexen interkulturellen Sinnzuschreibungen (s. Kap. 2.1, 2.2). 


\section{Sinnebenen des Fremdsprachenunterrichts}

Rekonstruktive Fremdsprachenforschung kann als Teilgebiet der rekonstruktiven Sozialforschung betrachtet werden. Das Besondere der rekonstruktiven Fremdsprachenforschung liegt jedoch eindeutig in der Einbeziehung des - partiellen oder sogar durchgängigen - Gebrauchs der fremden Sprache. Dieser Sprachgebrauch kann auch bei einigen untersuchten Konstellationen im Rahmen der rekonstruktiven Sozialforschung auftauchen, aber eben in der Regel nicht in intendierter bzw. sogar institutionalisierter Weise. Dieser intentionale und institutionalisierte Charakter bedingt auch bei der Arbeit mit der Dokumentarischen Methode einige Veränderungen, die hier im Vorgriff bereits genannt werden sollen. Zu den Hauptsinnebenen, die mit der Dokumentarischen Methode untersucht werden, dem kommunikativen (meist expliziten ${ }^{6}$ ) und dem konjunktiven (impliziten ${ }^{7}$ ) Sinn (s. auch Kap. 3.1.3), tritt nämlich noch der intendierte Sinn (meist Ziel und Zweck einer Aufgabe, aber auch der Bezug von LehrLernhandlungen auf institutionelle Vorgaben wie Lehrpläne und Evaluationen) und der individuelle lernersprachliche Sinn hinzu. Letzterer verkompliziert das Fremdsprachenlernen außerordentlich, wie oben bereits mit dem Hinweis auf Vygotsky angedeutet wurde: Es treten den Gebrauch der fremden Sprache betreffende Aushandlungsprozesse zu den ohnehin meist komplexen thematischen Aushandlungsprozessen hinzu. Natürlich kann die Sprache selbst auch Thema des Aufgabensinns und der davon initiierten Sinnebenen sein. Auf alle Fälle laufen im Fremdsprachenunterricht ständig im Hintergrund individuelllernersprachliche Konstruktionsprozesse ab, die die Verständigung beeinflussen können.

Der lernersprachliche Sinn steht im Zusammenhang mit individuellen Entwicklungsständen in der Lernersprache sowie der mehr oder weniger entwickelten Sprachbewusstheit der Beteiligten. Das heißt, er kann vor- oder unbewusst sein und sich bloß performativ, d.h. in der praktischen Anwendung äußern; er kann allerdings auch reflektiert und dadurch bewusst werden. Hier schließt sich

6 Auf dieser Sinnebene bleibt der Forscher noch innerhalb der Relevanzsysteme der Beforschten, daher wird diese Sinnebene auch „immanenter“ Sinn genannt. Auf der Wissensebene spricht man vom „kommunikativen Wissen“ oder auch von „explizitem Wissen".

7 Auch als „exmanenter Sinn“ bezeichnet: Der Forscher verlässt die Relevanzsysteme der Beforschten. Bohnsack spricht in dieser Hinsicht vom „dokumentarischen Sinn“ und spielt damit auf die von ihm entwickelte rekonstruktive Methode an. Auf der Wissensebene wiederum werden die Bezeichnungen „implizites Wissen“ oder in Anlehnung an Mannheim „konjunktives“ bzw. „atheoretisches Wissen“ verwendet. 
somit aus Forscherperspektive eine Untersuchung der Lernersprache an, die die üblichen Analyseschritte der Dokumentarischen Methode ergänzt, forschungsmethodologisch jedoch Fragen aufwirft (s. Kap. 2.1). Folgendes Schaubild fast die Sinnebenen der Fremdsprachenforschung mit der Dokumentarischen Methode bezogen auf fremdsprachenunterrichtliche Interaktionen auf einen Blick zusammen:

Tabelle 1: Sinnebenen des Fremdsprachenlehrens und -lernens

\begin{tabular}{|c:c|}
\hline $\begin{array}{c}\text { Intendierter Sinn } \\
\text { (meist Aufgabensinn) }\end{array}$ & $\begin{array}{c}\text { Individueller } \\
\text { lernersprachlicher } \\
\text { Sinn }\end{array}$ \\
\hline Kommunikativer Sinn & \\
\hline Dokumentarischer Sinn & \\
\hline
\end{tabular}

Die unterbrochene Linie deutet an, dass der lernersprachliche Sinn in einer Interaktion mit den drei anderen Sinnebenen steht. Als intendierter Sinn ist er einer Aufgabe eingelagert; als kommunikativer Sinn zeigt er sich performativ oder auch reflexiv in der Aufgabenbearbeitung; als dokumentarischer Sinn zeigt er sich in den Rahmenorientierungen der Lernenden, z.B. in einer Rahmung als Sprachübung oder im Gegenteil in einer Peer-orientierten Ablehnung der Sprachübung (s. Kap. 2.1).

Den verschiedenen Sinnebenen des Fremdsprachenunterrichts sind selbstredend unterschiedliche Wissensebenen zugeordnet. Der Aufgabensinn steht in direktem Bezug zum fachdidaktischen Wissen, etwa in Form einer bestimmten Kompetenzmodellierung. Der kommunikative Sinn steht in Bezug zum Kommunikationswissen bzw. den common grounds einer Verständigung, etwa in Form der Gesprächsregeln im Unterricht oder der inhaltlichen Beantwortung einer Lehrerfrage. Der konjunktive Sinn steht in direktem Bezug zum atheoretischen Wissen der Beteiligten über ihr Alltagshandeln, d.h. ihren Rahmenorientierungen (etwa der Rahmung durch eine Peer-Konstellation). Und der lernersprachliche Sinn schließlich steht im Bezug zum bewussten und unbewussten Sprach(en)wissen der Beteiligten, d.h. ihrer performativ sich äußernden Kenntnis etwa bestimmter fremdsprachiger Lexik oder ihrer Sprachbewusstheit 
bezogen auf sprachliche Regeln und Systeme, auf Register und Varietäten sowie auf sprachliche Beeinflussung.

\section{Verständigungsbereiche des Fremdsprachenlehrens und -lernens}

Im Folgenden werden nun die verschiedenen sinngenerierenden Verständigungsbereiche des Fremdsprachenlehrens und -lernens in den Blick genommen. Die Verständigung im Kollektiv der schulischen oder außerschulischen Fremdsprachenlerngemeinschaft kann wie oben bereits angedeutet auf vier Ebenen untersucht werden:

- die Verständigung durch Texte,

- die Verständigung über Texte (i. d. R. durch Aufgaben),

- die Verständigung über den Unterricht zu Texten,

- die Verständigung über Unterricht.

Alle vier Ebenen können als Konstruktionsebenen betrachtet werden, insofern auf allen vier Ebenen kollektive Konstruktionsprozesse stattfinden, die rekonstruiert werden können. Im Rahmen des schulischen Fremdsprachenlernens sind sie in der Regel in Aufgabenbearbeitungsprozesse eingebettet. Somit finden neben den vier Konstruktionsprozessen drei Transformationsprozesse statt: Die Transformation eines Textes in einen intendierten Sinn (= Aufgabenerstellung), die Transformation eines intendierten Sinnes in einen realisierten Sinn (= Aufgabenbearbeitung) und schließlich die Transformation des realisierten Sinnes in Metakonzepte des Lernens (Evaluation von Unterricht).

Im direkten Anschluss werden die vier Verständigungsbereiche kurz vorgestellt und in den Folgekapiteln detailliert beschrieben.

\section{a) Verständigung durch Texte}

Gemeint ist die Text-Rezipient-Interaktion, d.h. die Ziele und Mittel, die Textproduzierende bei der Gestaltung von Texten einsetzen, sowie die Wirkung, die der Text bei Rezipienten auslöst. Die Sinnkonstruktion wird hier einerseits als Textstruktur und andererseits als Rezeptionsstruktur sichtbar.

Text
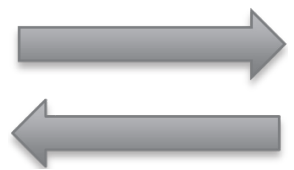

Rezipient 
Beispiele dafür sind etwa die formalen und inhaltlichen Strukturelemente eines politischen Fotos oder die narrativen und stilistischen Strukturen eines Erzähltextes. Diese Sinnebene wird in Kapitel 2.2 näher erläutert.

\section{b) Verständigung über Texte}

Gemeint ist die Anschlusskommunikation unter den Rezipienten eines Textes über den Text, z.B. ein Foto oder einen Erzähltext, an Hand bestimmter Aufgabenstellungen. Diese Verständigung kann man in der Terminologie Vygotskys als Mediation (Vermittlung) bezeichnen (zur Diskussion um den Begriff der Vermittlung s. Wertsch 2007).

Text

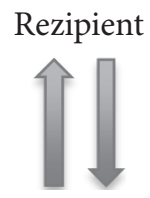

Rezipient

Aufgaben, Materialien (Lehrmaterialien, Texte, Medien) und Personen (Lehrende, Lernende) sind potenzielle Mediatoren, aber auch das gesamte lernrelevante Vorwissen (vorgelernte Sprachen, Weltwissen, didaktisches Wissen). Sprachliche Verständigung in der Interaktion verläuft meist in einer dreiphasigen Diskursbewegung aus Orientierung, Ausführung und Kontrolle (Lantolf \& Poehner 2008: 48), oder - in der Sprache der rekonstruktiven Sozialforschung - aus Proposition, Ausführung und Konklusion (Pzyborski 2004: 59ff.).

Forschungsfragen beziehen sich zum Beispiel auf den Stellenwert von Interimstexten oder Zwischenergebnissen: Wie gehen Unterrichtende mit Normabweichungen in sprachlichen (Zwischen-)Produkten Lernender um? Wie bewerten sie sie, wie melden sie deren Qualität zurück? Wie gehen Lernende mit ihren Interimstexten um und mit den Interimstexten der Mitlernenden? Wie bewerten sie sie, welche darauf bezogene Bewertung fordern sie ein, wie geben sie ihre Bewertung an die Peers und an die Unterrichtenden weiter? Welche Anschlussverständigung findet auf Grund des Feedbacks statt? Derartige Rezeptionsgespräche wurden beispielsweise von Michel (2010) (allerdings bezogen auf den nichtfremdsprachlichen Unterricht), von Tesch (2010), von DeckeCornill (2013) oder auch von Bracker (2015) dokumentarisch untersucht. Sie erweitern das Spektrum der Aufgabenbearbeitungsforschung, die in den letzten Jahren vermehrt in den Blick der Allgemeinen Didaktik, wie auch der Lehr- und 
Lernforschung sowie der Fachdidaktik gewandert ist (s. z.B. Thonhäuser 2008). Die Verständigung durch Aufgaben wird in Kapitel 2.3 näher erläutert.

\section{c) Verständigung über den Unterricht}

Gemeint ist die Evaluation einer oder mehrerer Unterrichtssequenzen durch die Lernenden beispielsweise im Rahmen eines separaten Plenumsgesprächs im Unterricht oder auch eines Interviews oder Gruppengesprächs nach dem Unterricht. Auch in dieser Verständigung kann die gemeinsame Konstruktion von Sinn als sozial geteiltes Wissen über Unterricht untersucht werden, und die kleinste Untersuchungseinheit besteht wieder in der dreiphasigen Diskursbewegung aus Proposition, Ausführung und Konklusion. Derartige Evaluationen wurden u. a. von Tesch (2010) untersucht. Ihnen ist Kapitel 2.4 gewidmet.

\section{d) Verständigung über das Lehren und Lernen von Sprachen}

Die Mesoebene der Evaluation spiegelt sich z.B. in der Evaluation eines Seminars durch Studierende oder in der Fachkonferenz an einer Schule zum Thema kompetenzorientierter Fremdsprachenunterricht (s. Tesch 2010; für den Mathematik-, Deutsch- und Englischunterricht sowie die Naturwissenschaften vgl. Zeitler, Heller \& Asbrand 2012). Auch narrative Interviews mit Lehrerinnen und Lehrern können mit Hilfe der Dokumentarischen Methode ausgewertet werden (zu den erforderlichen Modifikationen s. Kap. 3.2.).

Die Verständigung über das Lernen von Sprachen: Im Zentrum des Interesses stehen hier individuelle Sprachen- und Sprachlernbiografien, die mit Hilfe des narrativen Interviews analysiert werden können. Die beiden Aspekte der Verständigung über das Lehren und über das Lernen von Sprachen werden ebenfalls in Kapitel 2.4 theoretisch erläutert und in Kapitel 3 praktisch angeleitet.

\section{Nutzungsmöglichkeiten rekonstruktiver Fremdsprachenforschung}

Zum Abschluss dieser Einleitung gehe ich auf die Frage der Nutzungsmöglichkeiten der rekonstruktiven Fremdsprachenforschung mit der Dokumentarischen Methode ein.

Es liegt nahe, zunächst an wissenschaftliche Abschluss- und Qualifikationsarbeiten zu denken. Tatsächlich liegen bereits zahlreiche interessante Arbeiten, z. B. an den Lehrstühlen für die Didaktik des Englischen an der Universität Hamburg und für die Didaktik des Französischen und Spanischen an der Universität Kassel, vor. Dabei lassen sich theoretisch zwei Grobzielrichtungen bestimmen: 
a) Begleitforschung zur Implementation allgemeinpädagogischer und didaktischer Konzepte (z.B. Bonnet 2004 zum bilingualen Sachfachunterricht; Tesch $2010 \mathrm{zu}$ Kompetenzorientierung und Bildungsstandards);

b) Die Weiterentwicklung von Theorie im Hinblick auf spezifischere fremdsprachendidaktische Konzepte (z.B. Bracker 2015 zur literarischen Anschlusskommunikation).

Weitere Forschungsfragen wären z.B. die Nutzung von Mehrsprachigkeitspotentialen oder genrespezifische Verstehensprozesse etwa im Hinblick auf Comic oder Film und Aufgabenbearbeitungspraxen unter den Bedingungen von Inklusion und Migration. Ganz allgemein kann die Theorie des fremdsprachlichen Klassenzimmers zum Gegenstand rekonstruktiver Forschung gemacht werden.

Doch auch in den Phasen der Lehrerbildung kann sie nutzbringend in Forschungsseminaren und Lehrerfort- und Weiterbildungen eingesetzt werden (vgl. Bonnet 2009). Der Wechsel der Analyseeinstellung vom Was zum Wie bringt eine Fülle von Erkenntnissen über das Lehren und Lernen von Sprachen, kann zur Erhöhung der Selbstreflexivität führen und ist z.B. geeignet, die für viele Praktiker noch immer abstrakt gebliebene Kompetenzorientierung transparenter werden zu lassen. Die Dokumentarische Methode stellt ein Analysewerkzeug für Texte aller Art bereit. Die Analyse von Unterricht unter den Blickwinkeln „Was wurde gesagt“ und der Wechsel zu „Wie wurde es gesagt“ ermöglicht es, Performanzen rekonstruktiv zu evaluieren, ,Ergebnisse' praxeologisch zu interpretieren und auf diese Weise das Unbehagen vieler Praktiker an einer pädagogischen Begrifflichkeit, die oft als leer empfunden wird (Keutel \& Grossarth 2015), zu beseitigen und abstrakte Begriffe in erfahrbare Praxis zu transformieren. Erfolgt eine wissenschaftliche Begleitung dieser Erforschung des eigenen Unterrichts, kann man von Aktionsforschung sprechen (Altrichter \& Posch 1998). Bonnet (2009: 237) fasst zusammen:

Aufgrund dieser sehr differenzierten Betrachtung von Unterricht, die die Dokumentarische Methode ermöglicht, eignet sie sich schließlich hervorragend für die Anwendung in einer fallorientierten Lehrerbildung. Sie richtet den Blick auf die Handlungsstrukturen und damit auch auf jene Personen, die diese Strukturen beeinflussen. Dadurch wird eine differenzierte Betrachtung des Wechselspiels von Planungsentscheidungen, Interaktionssteuerung im Verlauf und Wirkungen auf verschiedenen Ebenen möglich. Dies kann durch zusätzliche Interviews mit Lehrenden und Lernenden vertieft werden. [...] Dies ermöglicht es angehenden aber auch im Beruf stehenden Lehrerinnen und Lehrern, in Unterrichtsanalysen eigene Überzeugungen über die Prinzipien und Funktionsweisen von Unterricht zu entwickeln bzw. auf den Prüfstand zu stellen. 
Mit dieser Einleitung wurden die Grundlagenkonzepte rekonstruktiver Fremdsprachenforschung eingeführt, nämlich der Wissensbegriff und der Begriff der Rekonstruktion von Wissen nach Mannheim, das Konzept der Sinnebenen des Fremdsprachenunterrichts sowie alles umrahmend der praxeologische Ansatz dieser Forschung. Die Sinnkonstruktion wurde als ihre anthropologische Grundannahme ausgewiesen und es wurden Bezüge zur Lerntheorie Vygotskys und zur ursprünglich linguistischen Theorie der Lernersprache hergestellt.

Im nun folgenden zweiten Kapitel werden zunächst die grundlegenden methodologischen Bezüge der Dokumentarischen Methode verdeutlicht sowie auf die drei o. g. Verständigungsebenen im und zum Fremdsprachenlehren und -lernen näher eingegangen: die Verständigung durch den Text, die Verständigung über den Text (durch Aufgaben) sowie die Verständigung über das Lehren und Lernen von Sprachen. Im dritten Kapitel werden die Arbeitsschritte der Dokumentarischen Methode beschrieben, zunächst allgemein (Kap. 3.1) und dann spezifischer für das narrative Interview (Kap. 3.2). Im vierten Kapitel begeben wir uns in die ,Fallwerkstatt': An Hand einer Unterrichtssequenz zu einem Comic sowie zweier narrativer Interviews mit Junglehrerinnen werden Zwischenergebnisse von Analysen an konkreten Beispielen vorgestellt und bestimmte Aspekte diskutiert. Der Band schließt mit einem Ausblick auf die Perspektiven dokumentarischer rekonstruktiver Forschung (Kap. 5). 


\section{Sinnkonstruktion im Fremdsprachenunterricht}

\subsection{Methodologische Grundlagen}

In diesem Teilkapitel geht es darum, Fremdsprachenforschung mit der Dokumentarischen Methode im Kontext wissenssoziologischer, linguistischer und sprachlerntheoretischer Bezüge zu verorten. Die wissenssoziologische Verortung wurde von Bohnsack (2014) auf systematische Weise für die rekonstruktive Sozialforschung im Allgemeinen vorgenommen. Es liegt nahe, an diesen Text anzuknüpfen und davon ausgehend die Verbindung zur Fremdsprachenforschung zu suchen.

Das Kapitel ist so aufgebaut, dass ich zentrale Begriffe rekonstruktiver Sozialforschung vorstelle und sie im Hinblick auf die Fremdsprachenforschung ergänze, erläutere und reflektiere. Die Begriffe schließen auf Grund der inneren Logik rekonstruktiver Forschung aneinander an, die Reihung ist somit nicht zufällig. Ich gehe zunächst auf den Begriff des methodisch kontrollierten Fremdverstehens ein, bevor ich den Begriff der Diskursentwicklung im Fremdsprachenunterricht beschreibe und dann zur Indexikalität der Verständigung komme. Ich erläutere sodann den Begriff der Rahmenorientierungen, der Typenbildung und der Lernersprache. Letzterer schließt, wie in der Einleitung erwähnt, an die entsprechende linguistisch-fremdsprachendidaktische Theorie an, die hier mit dem soziologischen Konzept der Rekonstruktion verbunden wird. Ich gehe anschließend auf Lerngruppenmilieus und konjunktive Erfahrungsräume näher ein. Das Kapitel schließt mit Ausführungen zur Validität rekonstruktiv ermittelter Ergebnisse und mit Überlegungen zu einem sozial-interaktionalen Modell des fremdsprachlichen Lehrens und Lernens.

\subsubsection{Methodisch kontrolliertes Fremdverstehen}

Rekonstruktive Verfahren greifen so wenig wie möglich in die Entstehensbedingungen der Verständigung ein und beschränken sich darauf, Deutungen zu rekonstruieren bzw. die Unterschiede der Deutung zu benennen. Folgender Vergleich rekonstruktiver und standardisierter Verfahren verdeutlicht, was gemeint ist. Soll beispielsweise die Verständigung in einer Gruppenarbeit zu einem Bildimpuls im Französischunterricht einer achten Klasse untersucht werden, so würde es die Anwendung eines standardisierten Verfahrens erforderlich machen, die Entstehungsbedingungen zu ,kontrollieren', d.h. man würde sämtliche 
Außenfaktoren der Aufgabenbearbeitung wie Arbeitsanweisung, Feedbackgeber (z.B. die Lehrperson), Bearbeitungszeit und Bearbeitungsmedien (z.B. ein Arbeitsblatt) als sogenannte unabhängige Variablen konstant halten müssen, um von allen Gruppen Bearbeitungsergebnisse (abhängige Variablen) unter gleichen Bedingungen zu erzielen. Diese könnten anschließend intersubjektiv überprüfbar verglichen werden. Bei rekonstruktiven Verfahren hingegen würde man im Gegenteil keinerlei Eingriffe in den natürlichen Ablauf vornehmen und die freie Entwicklung der verschiedenen Interaktionen bzw. der Gruppengespräche beobachten. Ziel ist es hier, Relevanzbestimmungen in den Lerngruppen nicht von außen zu beeinflussen. Ein teilnehmender Beobachter würde sich zusätzlich eine Vorstellung von der Stimmung zum Zeitpunkt der Untersuchung machen, etwa dem Druck einer bevorstehenden Mathematikarbeit in der nächsten Unterrichtsstunde, die bereits ihre Schatten vorauswirft und dazu führt, dass einige Schüler vielleicht heimlich Mathematikaufgaben besprechen.

Der Forscher würde sich als nächstes gezielt seinem Forschungsgegenstand gegenüber ,fremd' machen, sich fragen, wie er die Äußerungen der beobachteten Schülerinnen und Schüler versteht und dieses Eigenverstehen mit dem Verstehen anderer Personen abgleichen. In Forschungsseminaren zu Unterrichtstransskripten fiel mir immer wieder auf, wie unterschiedlich Studierende bestimmte Episoden interpretierten, sowohl untereinander als auch im Vergleich zu meiner eigenen Interpretation. Obwohl ich als ehemaliger Fremdprachenlehrer mit über zwanzigjähriger Unterrichtserfahrung viele Episoden gut einzuschätzen glaubte, hatten Studierende bei manchen Episoden eine plausiblere Deutung, oft dem Umstand geschuldet, dass sie auf Grund der geringeren Altersdifferenz das Verhalten der Schülerinnen und Schüler, deren Lebenswelt (z.B. Umgang mit bestimmten elektronischen Medien) und deren Sprache besser verstanden. Es entstand so eine völlig unterschiedliche Fremdheitsrelation unter den Interpreten und dem zu Interpretierenden gegenüber. Das Beispiel macht deutlich, dass die methodische Kontrolle bei interpretativen Verfahren in einer Kontrolle des Fremdverstehens durch die kommunikative Validierung innerhalb einer Forschergruppe liegt. Man spricht daher auch von methodisch kontrolliertem Fremdverstehen ${ }^{8}$ (Bohnsack 2014: 21).

Der Mehrwert rekonstruktiver Verfahren gegenüber standardisierten Verfahren der Fremdsprachenforschung liegt auf der Hand: Es können mit ihrer Hilfe Interaktionen methodisch kontrolliert analysiert werden, die sich ohne

8 Auch in der Fremdsprachendidaktik gibt es den Begriff des Fremdverstehens. Bredella und Christ z. B. benutzten ihn 1995 in ihrer „Didaktik des Fremdverstehens“ (s. Kap. 2.2). 
äußerliche Beeinflussung oder Vorstrukturierung entfalten können. Bohnsack spricht von der freien Entfaltung des „Relevanzsystems“ (ibd.: 22). Damit können diese Verfahren dem Forschungsgegenstand der Interaktion im Alltag des Fremdsprachenunterrichts als in besonderem Maße angemessen betrachtet werden. Das Ziel der Dokumentarischen Methode ist die Erforschung von Relevanzsystemen und deren Regelhaftigkeiten.

Indem sich der Blick des Forschenden vom Was der Kommunikation auf das Wie richtet, kann ein Wissen transparent werden, das den Beforschten selbst meist nicht bewusst ist: Den Schülerinnen und Schüler selbst ist nicht bewusst, wie sie miteinander kommunizieren. Der Fremdsprachenforscher kann jedoch herausfinden, wie sie z.B. ein Bild beschrieben haben, welche humorvollen, selbstkritischen, ironischen etc. Bemerkungen dabei gemacht und wie die sprachlichen Hürden gemeistert wurden. Aus diesen Befunden ergibt sich auch eine Regelhaftigkeit im Kontext der Gesamtunterrichtsstunde, die bei der Betrachtung eines isolierten Ausschnitts möglicherweise verloren gegangen wäre.

\subsubsection{Diskursentwicklung im Fremdsprachenunterricht}

Viele linguistische Interaktionsanalysen zum Fremdsprachenunterricht beschränkten sich auf kurze Ausschnitte, auf wenige turns ${ }^{9}$. Dabei geriet der Kontext, in dem die Äußerungen geschahen, meist aus dem Blick. Batstone \& Philp (2013: 109) äußern dazu:

In our view, the nature of negotiation (like any form of interactive discourse) changes depending on the contexts in which it is situated. Classroom negotiation will inevitably be shaped by its social and pedagogic context in fundamental ways. In order to better understand interaction-driven learning, we need to pay more attention to the complexity of classroom interaction and its many facets.

Aus unserer Sicht verändert sich die Art der Aushandlung (wie jede Form interaktiven Diskurses) in Abhängigkeit der Kontexte, in denen sie situiert ist. Aushandlungen im Klassenzimmer werden unweigerlich durch ihren sozialen und pädagogischen Kontext fundamental beeinflusst. Um interaktionsbasiertes Lernen besser zu verstehen, müssen wir der Komplexität der Klassenzimmerinteraktion und ihren zahlreichen Facetten mehr Aufmerksamkeit widmen. (Übers. BT)

9 Unter turn (Sacks et al. 1974) versteht man in der Konversationsanalyse meist die kleinste Untersuchungseinheit: Ein Sprecher ergreift das Wort, reagiert ggf. auf eine vorangegangene Äußerung und macht selbst eine Äußerung, auf die dann andere wieder reagieren können. Bohnsack \& Przyborski (2006: 233f.) setzen sich im Rückgriff auf Goffman (1981) kritisch mit der Untersuchung isolierter turns auseinander. 
In der rekonstruktiven Fremdsprachenforschung mit der Dokumentarischen Methode wird die Entwicklung des Diskurses über eine gesamte oder sogar mehrere Unterrichtsstunden in den Blick genommen. Bestimmte Deutungshypothesen sind erst im Handlungskontext des Gesamtdiskurses plausibel. In diesem Blick auf das Ganze enthalten sind die Parallelgespräche und Nebenthemen des fremdsprachlichen Klassenzimmers sowie Lernabbrüche und -unterbrechungen durch unvorhergesehene Ereignisse. Das folgende Beispiel verdeutlicht das bisher Gesagte.

Es handelt sich hier um einen Auszug aus dem vollständigen Stundentranskript einer Spanischstunde in einer achten Gymnasialklasse. Die Lerner befanden sich im zweiten Lernjahr und sollten u. a. verschiedene Personen beschreiben ${ }^{10}$.

Im Text werden vier Personen genannt: Javi, Sarah, Esteban und Elena. $\mathrm{Zu}$ jeder Person gibt es fünf Felder, die von jedem Schüler bearbeitet werden mussten: ¿Cómo es?, asignatura favorita, también es bueno/-a en, no es bueno/-a en, actividades después de las clases. (Wie ist er/sie? Welche sind seine/ihre Lieblingsfächer? Er/Sie ist auch gut in ... / Aktivitäten nach dem Unterricht). Nachdem die Schüler in Einzelarbeit diese fünf Felder bearbeitet hatten, sollten sie diese ihren Gruppenmitgliedern vorstellen. Die Gruppenmitglieder hatten die Aufgabe, die fehlenden Informationen von den drei verbleibenden Personen mit Hilfe eines ,Experten` zu ergänzen (Gruppenpuzzle). Als nächstes sollten sie die untere Tabelle mit Hilfe des Textes vervollständigen. Abschließend hatten die Schülerinnen und Schüler die Aufgabe, eine Regel zum Gebrauch von ser und estar, den beiden spanischen Verbformen für „sein“, herauszufinden. Somit entstand ein etwa 25-minütiges Gruppengespräch. Nach der Gruppenarbeitsphase wurden die Ergebnisse im Plenum vorgestellt.

Klasse 8, Gruppe Blau, Passage „Esteban“, Z.69 bis 119, 3.46” bis 5’42” (Zeilennummerierung im Teiltranskript):

10 Der Arbeitsauftrag für die Unterrichtsstunde umfasste zwei Arbeitsaufträge. Primero: Formad grupos nuevos con expertos de cada persona; presentad a vuestra persona y el resto del grupo rellena los cuadros con otras informaciones. Segundo: Rellenad juntos la tabla de abajo con la ayuda del texto, descubrid una regla para el uso de „estar-adjetivo“ $y$ „ser-adjetivo“. Dazu sollte im Lehrbuch ¡Apúntate! (Cornelsen 2008: 89) der Text Así son los chicos gelesen werden. Dann sollte jedes Gruppenmitglied eine im Buch beschriebene Person heraussuchen und diese in Still- und Einzelarbeit mit einem Arbeitsblatt bearbeiten. 


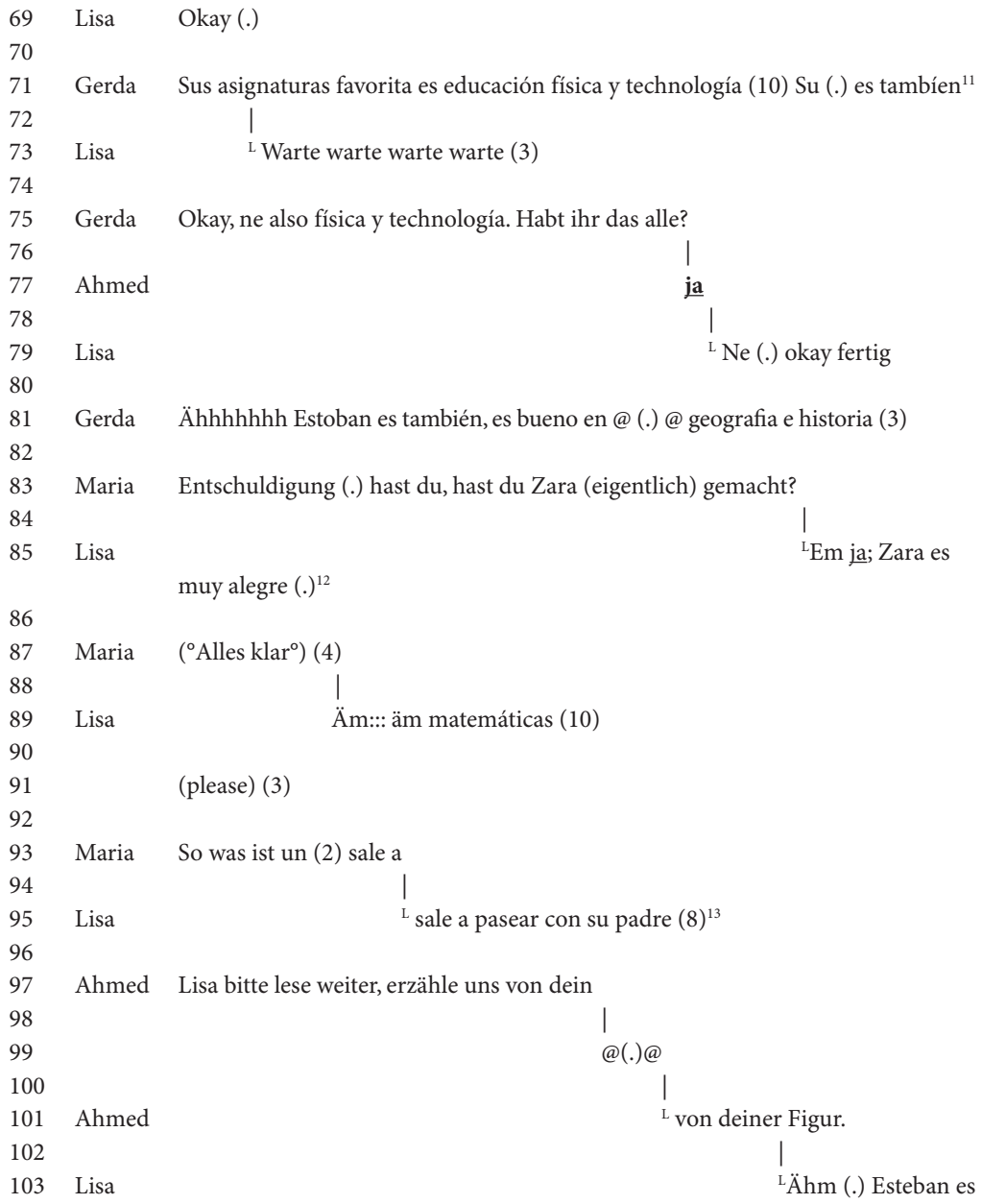

no bueno en mates; oder wie man das richtig formuliert.

11 „Seine Lieblingsfächer ist Sport und Technik. Sein (.) ist auch“.

12 „Zara ist sehr lustig“.

13 "geht mit ihrem Vater spazieren“. 


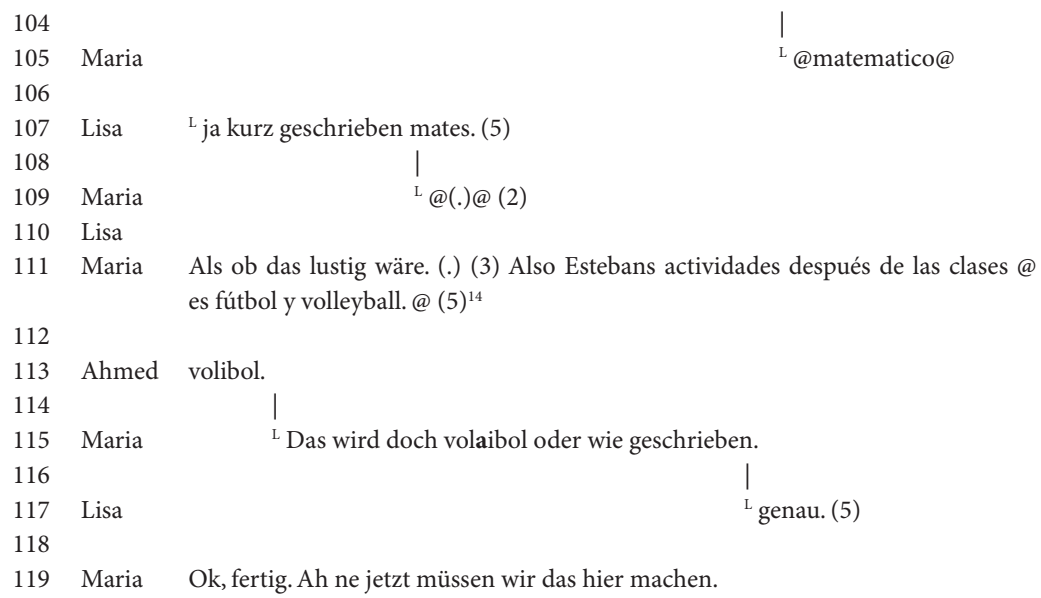

Fassen wir einmal die Teilsequenz ab Z. 97 näher ins Auge. Sie zeigt auf der lernersprachlichen Ebene folgende Diskursbewegungen:

i. Ahmeds Orientierung verbunden mit einer Aufforderung an Lisa zur Weiterarbeit („Lisa bitte lese weiter, erzähle uns von deiner Figur“, 97-101)

ii. Lisas Ausführung/Umsetzung („Esteban es no bueno en mates“, 103). Sie ist unsicher bezüglich der Wortstellung („oder wie man das richtig formuliert", 103)

iii. Marias Evaluation („@matematico@“,105)

iv. Lisas zweite Ausführung („Estebans actividades después de las clases @ es fútbol y volleyball. @“, 111)

Parallel dazu erfolgen folgende Diskursbewegungen auf der sozialen Ebene:

Ahmed übt die Moderatorenrolle aus, initiiert das Thema „Esteban“. Er fordert Lisa auf, Esteban zu beschreiben. Lisa proponiert ihre Variante der Aufgabenbearbeitung, zu der auch auf Deutsch metasprachlich das Zeigen einer Unsicherheit gehört („oder wie man das richtig formuliert“, 103). Maria korrigiert antithetisch einen vermeintlichen Fehler Lisas unmittelbar; dabei lacht sie ein wenig. Lisa reagiert sofort darauf, indem sie Marias Lachen als Auslachen deutet. Sie kommentiert ihren Umsetzungsversuch auf Deutsch (,ja kurz geschrieben mates“, 107) und verlässt mit diesem Sprachwechsel (code-switching) die Sprachübung bzw. droht eine Aufkündigung des gemeinsamen Rahmens:

14 „Estebans Aktivitäten nach dem Unterricht sind Fußball und Volleyball“. 
„Als ob das lustig wäre“ (111). Nach einer kurzen Pause kehrt sie jedoch in den gemeinsamen Rahmen zurück. Dazu verwendet sie das Übergangssignal „also“ (111), und fährt mit einer Anschlusselaboration zum Thema „Esteban“ fort. Ihr Kichern könnte so gedeutet werden, dass sie bezüglich der Aussprache unsicher ist und um diesbezügliche Hilfe bittet. Ahmed validiert die Aussprache des Wortes („volibol“, 113). Diesmal erweitert Maria das Thema „Esteban“ durch eine Anschlussproposition zum Subthema „korrekte Schreibweise“ im Modus einer Frage. Lisa validiert die Schreibweise, und Maria konkludiert das Thema im Modus einer rituellen Synthese („Ok, fertig.“, 119).

Die reflektierende Feinanalyse der Passage bringt zum Vorschein, wie die zielsprachliche Bedeutungsaushandlung durch eine soziale Interaktion überlagert wird. Wäre die Interpretation auf der Ebene des Was, d.h. des kommunikativen Sinns, geblieben, so wäre der latente Konflikt zwischen Lisa und Maria verborgen geblieben. Es wird daran deutlich, wie eng das sprachliche Bedeutungsaushandeln verknüpft ist mit dem Aushandeln einer gemeinsamen sozialen Orientierung, die darin besteht, den Rahmen der Sprachübung letztlich zu wahren. Auf der Hinterbühne kämpfen die Peers jedoch um ihre Position. Die inkludierende Rahmung der Aufgabenbearbeitung wird partiell überlagert durch ebenfalls inkludierende Modi der Peerbeziehungen.

Ohne Kenntnis der obigen Textpassage wäre auch die folgende Episode zum Thema Mathe-Aufgabe nicht zu verstehen (Zeilennummerierung im Gesamttranskript):

$\begin{array}{lll}108 & \text { Gerda } & \text { Ich hab seit einem halben Jahr mit Matheproblemen zutun. } \\ 109 & \text { Maria } & \text { Jaa, warte mal ganz kurz. Oh ich hab doch auch kein Lust. } \\ 110 & \text { Gerda } & \text { Hä du hattest doch die übelste Arbeit in Mathe. } \\ 111 & \text { Lisa } & \text { Matheschwäche? Oh ich hab auch ne Matheschwäche. } \\ 112 & \text { Maria } & \text { Ok also ja Javier es simpática. } \\ 113 & \text { Gerda } & \text { Ahmed nicht, Ahmed ist ja eh der Schlauste aus der Klasse } \\ 114 & \text { Lisa } & \text { Der (Mathelehrer) hat mich gefragt, ob ich überhaupt schon mal ein } \\ 115 & & \text { richtiges Buch gelesen hab. Ich bin noch sauer } \\ 116 & \text { Gerda } & \text { Javi es } \\ 117 & \text { Ahmed } & \text { Hä wieso hat der das gefragt? Wer hat den heutzutage noch nie ein } \\ 118 & & \text { richtiges Buch gelesen. }\end{array}$

Von außen betrachtet würde dieses Thema völlig überraschen, da es nichts mehr mit der Sprachübung zu tun zu haben scheint. Die Diskursanalyse offenbart jedoch den sozialen Zusammenhang: Lisa stellt in der Sprachübung Esteban vor, der nicht gut in Mathe ist. Das „Nicht-Gut-Sein in Mathe“ beschäftigt die Mädchengruppe unbewusst, so dass das Thema ein paar Diskursbewegungen weiter scheinbar plötzlich und nur scheinbar unvermittelt wieder auftaucht. In 
Wirklichkeit hat sich die Gruppe derart mit dem Inhalt der Sprachübung identifiziert, dass sie auf einer parallelen Bühne das Thema "Nicht-Gut-Sein in Mathe“ auf sich selbst bezieht und weiter elaboriert.

An diesem Beispiel ist gut zu erkennen, wie sich verschiedene Relevanzsysteme frei entfalten: Das Relevanzsystem der Peerbeziehungen in der Gruppe (Konflikt zwischen Lisa und Maria), die an einem bestimmten Punkt die weitere Aufgabenbearbeitung auszusetzen drohen, ferner das Relevanzsystem der Identifikation mit fiktiven Personen und deren Stärken und Schwächen (Estebans "Nicht-Gut-Sein in Mathe"). Die Lernersprache in der Gruppe bildet ein eigenes Relevanzsystem, dessen Entwicklung und Sinnstruktur sich zwar aus der Sprachübung (intendierter Sinn) ableiten lässt, jedoch durch die beiden sozialen Relevanzsysteme der Peerbeziehungen und der Identifikation mit fiktiven Personen beeinflusst wird. Es würde den Forschenden nicht weiter bringen, den Konflikt bei der Aufgabenbearbeitung mit einem den Beteiligten fremden Relevanzsystem interpretieren zu wollen, etwa mit dem Relevanzsystem eines Unterrichtenden, der Konflikte bei der Aufgabenbearbeitung grundsätzlich als Störung des Unterrichts betrachtet oder das Flüstergespräch auf Deutsch zum Thema „NichtGut-Sein in Mathe" als Ablenkung vom Eigentlichen.

Wie in Kapitel 2.1.1 ausgeführt bedeutet methodische Kontrolle in der rekonstruktiven Forschung die

Kontrolle über die Unterschiede der Sprache von Forschenden und Erforschten, über die Differenz ihrer Interpretationsrahmen, ihrer Relevanzsysteme. Und diese Kontrolle gelingt nur, wenn ich den Erforschten Gelegenheit gebe, ihr Relevanzsystem zu entfalten, und dann darauf aufbauend - rekonstruierend - mir die Unterschiede der Interpretationsrahmen vergegenwärtige.

(Bohnsack 2014: 22).

Der Weg zu dieser methodischen Kontrolle führt über die Rekonstruktion der Diskursorganisation, genauer gesagt ihrer verschiedenen Modi, worauf weiter unten (s. 3.1.3) näher eingegangen wird. Die rekonstruktive Sicht auf den Unterricht stellt die Theoriebildung zunächst zurück, bis sich eine Strukturierung des Forschungsgegenstandes durch die Beforschten selbst ergeben hat.

\subsubsection{Die Indexikalität der Verständigung}

Die Rekonstruktion der Relevanzsysteme beinhaltet jedoch nicht nur das Nachvollziehen modaler Beziehungen innerhalb der Verständigung, d.h. inkludierender und exkludierender Rahmungen, sondern auch der inneren Verweisstruktur von Äußerungen. Um beim obigen Diskursausschnitt zu bleiben: Der Forschende erkennt ebenso wie Lisa, dass Maria sie korrigiert und dass diese Korrektur 
vor dem Hintergrund einer (gespielten?) Rivalität geschieht. Doch bei Lisa wird daraus kein theoretisches Wissen, sie reflektiert es nicht, sondern sie handelt einfach entsprechend und droht, die gemeinsame Sprachübung aufzukündigen. Der Sprechakt, ,ja ich habe geschrieben mates. Als ob das lustig wäre“ verweist auf diese Rivalität. Lisa und Maria sind sich nicht gleichgültig. In ihrer (gespielten?) Rivalität haben sie eine bestimmte Beziehung zueinander und generieren somit gemeinsam und im Kollektiv der Gruppe Sinn. Dieser Verweischarakter von kommunikativen Handlungen wurde in der ethnomethodologischen Forschung von Garfinkel \& Sacks (1976) als „Indexikalität“ bezeichnet. Der Forschende kann die Indexikalität dieser Rivalität aufklären, indem er z.B. den Gegenhorizont nicht erfolgter sprachlicher Korrekturen heranzieht, wie er in anderen Fällen von Gruppenarbeit beobachtet werden kann. Kommunikativ betrachtet hätte nämlich gar keine Notwendigkeit für diese lernersprachliche Korrektur bestanden.

In diesem Zusammenhang stellt sich die Frage, in welchem Verhältnis das Wissen der Beforschten zum Wissen der Forschenden steht und, weiterführend, welches spezifische Wissen durch eine Bestimmung dieses Verhältnisses generiert werden kann. In der Wissenssoziologie Karl Mannheims wird das Alltagswissen der Beforschten als atheoretisch bezeichnet in dem Sinne, dass es ein intuitives unmittelbares Verstehen ermögliche. Es sei prinzipiell jedoch strukturidentisch mit dem Wissen der Forschenden, das heißt die Produktionsregeln der Verständigung seien prinzipiell die gleichen. Der Forschende sei dadurch auch prinzipiell in der Lage, die Konstruktionsgesetzmäßigkeiten der beforschten Verständigung zu rekonstruieren. Nur geschieht dies bei ihm nicht intuitivunmittelbar sondern reflexiv, auf dem Wege der reflektierenden Interpretation. Der Forschende kann, vom unmittelbaren Handlungsdruck befreit, den geäußerten Haltungen Vergleichs- oder Gegenhorizonte entgegensetzen, sie dadurch schärfer konturieren.

\subsubsection{Rahmenorientierungen}

Die Rekonstruktion der Diskursorganisation im Hinblick auf ihre Modi und ihre innere Verweisstruktur erlaubt es nun, das implizite Wissen der Beforschten sichtbar werden zu lassen und es gemeinsamen Rahmenorientierungen oder einfach: Rahmungen, zuzuordnen. Wie bereits oben ausgeführt handelt, es sich dabei um Re-Konstruktionen, also um die Wieder-Herstellung von Konstruktionen, die bereits von anderen Personen generiert wurden, ihnen aber in der Regel nicht bewusst sind. Auch bei der Verständigung im Fremdsprachenunterricht finden sich wie in anderen Lebensbereichen nicht nur geteilte Orientierungen, 
sondern auch nicht geteilte. Die Anzahl der Rahmungen ist im Fremdsprachenunterricht jedoch nicht unbegrenzt. So finden wir bei Lernenden stets einen thematischen Rahmen, innerhalb dessen sie sich bezüglich persönlicher Relevanz orientieren, auch andere Perspektiven einnehmen, oder eben nicht. Wir finden ferner meist eine Rahmung in Form einer Sprachübung, die die Lernenden entweder im Sinne individueller Progression annehmen oder nicht, dabei Autonomie entwickeln oder nicht und sich an ihren individuellen Selbstwirksamkeitserwartungen (Bandura 1997) orientieren. Wir finden eine Rahmung durch lernaktive Ko-Konstruktion oder durch Kooperationsverweigerung. Wir finden weiter einen Rahmen in Form eines bestimmten Aufgabenkonzepts. Letzteres kann z.B. ein einfaches Übungskonzept sein (z.B. ppp: preparation, practice, presentation) oder task-based learning, also ein Lernen an komplexen, möglichst lebensnahen Aufgaben. Lernende orientieren sich innerhalb dieses Rahmens z.B. eher am Produkt (z. B. ein Poster) oder am Prozess seiner Entstehung, an Verantwortungsübernahme oder an Verantwortungsabgabe, an der Aufgabenerfüllung mit Blick auf den intendierten Sinn oder an bloßer Aufgabenerledigung. Darüber hinaus findet sich in den Daten mitunter der Rahmen der sozialen Einbindung - Lernende orientieren sich z.B. an Rahmungen ihrer Familie, ihres Kiezes, ihrer Peers - und eine Rahmung durch die einfache Macht der Gewohnheit (z.B. gewohnte Lehrmethodik, gewohnte Lernmethodik, gewohnte Materialien).

Das folgende Schaubild stellt verschiedene Rahmungen und Orientierungen im Fremdsprachenunterricht im Überblick dar. Die Rahmungen bilden den kollektiven Erlebnisraum ab, die Orientierungen die sozial geteilten oder nicht sozial geteilten individuellen Relevanzen:

Tabelle 2: Rahmungen und Orientierungen Lernender im Fremdsprachenunterricht (s. Tesch 2010, weiterentwickelt)

\begin{tabular}{|c|c|}
\hline Rahmung & Orientierung \\
\hline Thema, Text & $\begin{array}{l}\text { z.B. } \\
\text { - Individuelle Relevanz des Themas } \\
\text { - Individuelle Relevanz des Textes } \\
\text { - Perspektivwechsel } \\
\text { - Empathie }\end{array}$ \\
\hline Lernersprache und Spracharbeit & $\begin{array}{l}\text { z.B. } \\
\text { - Individuelle Progression/Leistungsmotiv } \\
\text { - Perfektion/sprachliche Korrektheit } \\
\text { - Aktivität/Partizipation } \\
\text { - Wartemodus/Passivität } \\
\text { - } \quad \text { individuelle Selbstwirksamkeitserwartung }\end{array}$ \\
\hline
\end{tabular}




\begin{tabular}{|c|c|}
\hline Rahmung & Orientierung \\
\hline Kooperation & $\begin{array}{l}\text { z.B. } \\
\text { - Aktive Ko-Konstruktion } \\
\text { - Kooperationsverweigerung }\end{array}$ \\
\hline $\begin{array}{l}\text { Aufgabenkonzept } \\
\text { (z.B. Übung, TBL, } \\
\text { Prüfungsaufgabe) }\end{array}$ & $\begin{array}{l}\text { z.B. } \\
\text { - Aufgabenerfüllung } \\
\text { - Aufgabenerledigung } \\
\text { - Verantwortungsübernahme } \\
\text { - Verantwortungsabgabe } \\
\text { - Produkt } \\
\text { - Prozess } \\
\end{array}$ \\
\hline Soziale Einbindung & $\begin{array}{ll}\text { z.B. } \\
\text { - } & \text { Familie } \\
\text { - Peers } \\
\text { - } \text { Kiez }\end{array}$ \\
\hline Gewohnheit & $\begin{array}{l}\text { z.B. } \\
\text { - } \text { gewohntes Lehrwerk } \\
\text { - } \text { gewohnte Lehrmethodik } \\
\text { - } \text { gewohnte Lernmethodik }\end{array}$ \\
\hline
\end{tabular}

\subsubsection{Typenbildung}

Letztendliches Ziel der dokumentarischen Analyse ist es, aus den empirisch ermittelten Orientierungen Typen zu bilden, um damit zu Aussagen größerer Reichweite zu gelangen. Rekonstruktiv gewonnene Ergebnisse unterliegen ähnlichen Gütekriterien wie empirische Ergebnisse auf Grundlage standardisierter Verfahren. Auch bei rekonstruktiver Forschung gibt es die Kriterien der Gültigkeit (Validität) und der Zuverlässigkeit (Reliabilität), doch beruhen sie auf anderen Voraussetzungen. Methodische Fragen werden in den Kapiteln drei und vier erörtert. An dieser Stelle begnüge ich mich mit einem Hinweis auf die Bedeutung des Fallvergleichs und der Typenbildung für die Gültigkeit von Ergebnissen. Stets handelt es sich bei empirischer Forschung um eine bestimmte Art des Vergleichs. Während bei standardisierten Verfahren Zahlenwerte verglichen werden, beruht der Vergleich bei rekonstruktiven Verfahren meist auf Diskurssegmenten, die interpretiert werden und als Fälle zusammenfassend beschrieben werden können. Damit aber nicht sozusagen ,Äpfel und Birnen` verglichen werden, bedarf es zweier Grundvoraussetzungen:

a) Es muss sich um homologe (gleichgelagerte) Fälle handeln (Bohnsack 2014: $221 \mathrm{f}$.).

b) Der Vergleich muss sich auf ein gemeinsames tertium comparationis beziehen, ein zu vergleichendes Drittes (ibd.). 
Zwei Beispiele verdeutlichen, was damit gemeint ist. Würde man die Vereinbarkeit von Familie und Beruf (= tertium comparationis) bei Grundschullehrerinnen und bei Hochschullehrerinnen vergleichen, so gäbe es bei klar definiertem tertium comparationis Ähnlichkeiten, aber auch deutliche Unterschiede bei den sozialen und materiellen Voraussetzungen der beiden Berufsgruppen. Die Fälle sind nicht homolog, da der konjunktive Erfahrungsraum fehlt. Eine konkrete grammatisch ausgelegte Lernsequenz im zweiten Lernjahr Spanisch (= tertium comparationis) einer hessischen Gesamtschule lässt sich zwar mit dem Unterricht ebendieser Grammatiksequenz in einer anderen Spanisch-Klasse im zweiten Lernjahr z. B. in Nordrhein-Westfalen vergleichen. Das tertium comparationis ist klar definiert, die Altersgruppe ist vergleichbar, der konjunktive Erfahrungsraum ist jedoch nicht ganz identisch. Ganz allgemein lässt sich dennoch sagen, dass der Makro-Erfahrungsraum Schule und Unterricht bei allen Spanischschülerinnen und -schülern sozial geteilt ist, selbst wenn das tertium comparationis so allgemein gefasst wäre wie ,der Spanischunterricht ${ }^{\text {}}$ an sich. Die Validität der Ergebnisse erhöht sich, wenn das tertium comparationis konkreter wird. Würde man die Bearbeitung des Comics Ne me quitte pas von Jordi Lafebre (s. Kap. 4.1) in einer elften Klasse eines hessischen Gymnasiums in Kassel mit der in einer elften Klasse in Hannover vergleichen, könnte der Vergleich bereits fokussiertere und in diesem Rahmen validere Ergebnisse erzielen.

Liegen nur Aufzeichnungen aus einer einzigen Lerngruppe vor (z.B. vier Lernende während einer Gruppenarbeit), so erfolgt der Vergleich fallintern. Das heißt, man kann verschiedene Episoden miteinander vergleichen, um Merkmale der Diskursentwicklung und darauf gründend Orientierungsmuster herauszuarbeiten. Nimmt man eine weitere Lerngruppe hinzu, erfolgt der Vergleich bereits fallübergreifend, und es wird gegebenenfalls möglich, erste Typiken zu bestimmen. Mit anderen Worten: Ist das sampling der ausgewählten Fälle sehr klein, so besteht das Risiko, dass die Theorie im Sinne der Grounded Theory (s.u.) nicht gesättigt ist, d.h. bestimmte theoretisch relevante Spuren unentdeckt oder unbearbeitet bleiben, oder dass keine Typenbildung erfolgen kann. Damit wiederum bleiben die Ergebnisse vielleicht auf der Ebene des Anekdotischen stehen. Ist das sampling hingegen für eine theoretische Sättigung ausreichend, können Typenschichtungen und darauf aufbauend Typologien bestimmt werden.

Grundprinzip der Typenbildung ist mithin das Vergleichen. Nur das ständige Vergleichen ermöglicht die Abgrenzung von Typen untereinander (s. Kap. 3.1.5). Wird beispielsweise in einer Lerngruppe eine Orientierung an „Aufgabenerledigung“ (statt „Aufgabenerfüllung“) ermittelt, so müsste dieser Befund mit weiteren Beobachtungen in derselben Lerngruppe, sodann in anderen Lerngruppen 
derselben Klasse, und schließlich mit Lerngruppen zum gleichen Thema in anderen Klassen verglichen werden. Aus diesem Vergleich resultiert die empirisch valide Abgrenzung und Bestimmung dieser gegenüber anderen Orientierungen und damit ihre sinngenetische Typenbildung. Im nächsten Schritt kann die soziogenetische Interpretation dieses Lernverhaltens erfolgen, z.B. aus der Organisation des Lernens in Gymnasialklassen heraus (Organisationstypik) oder aus einem schulstandorttypischen Umgang mit Lehrbüchern (Standorttypik, LehrLernmedientypik).

Je mehr Typiken in einer Fallauswahl ermittelt und empirisch abgesichert werden können, umso höher ist die Validität der Analyse. So konnte ich am Beispiel einer komplexen Lernaufgabe zur Förderung des zielsprachigen Sprechens, die in drei unterschiedlichen Klassen über mehrere Wochen hinweg unterrichtet wurde, eine solche geschachtelte Typologie fremdsprachenunterrichtlicher Rahmungen ermitteln (Tesch 2010).

Nohl (2013) stellt eine Möglichkeit vor, Typiken untereinander in Beziehung zu setzen bzw. Relationen zwischen verschiedenen Typiken zu bestimmen (Kap. 3.1.5). Dies erscheint bei stark ritualisierten Verständigungsformen wie dem schulischen Unterricht, innerhalb dessen eine begrenzte Anzahl bestimmter immer wiederkehrender Typiken sichtbar wird und die soziogenetische Typenbildung oft schwierig anzuwenden ist, als ein wichtiger Ansatz, die Spezifik der Fälle zu erkennen und darzustellen und mithin einen Beitrag zur angestrebten Theoriebildung zu leisten. Nohl (ibd.: 15-41) geht auch auf die Problematik des fallübergreifenden Vergleichens sozialer Gruppen - z. B.Lerngruppen in Schulen ein, d.h. von Gruppen, die zwar auf einer Ebene (hier: institutionelle Eingebundenheit in die Institution Schule) eine hohe Gemeinsamkeit aufweisen, also als homolog betrachtet werden können, auf anderen Ebenen (z.B. soziale oder ethnische Herkunft, kulturelles Kapital, fremdsprachliche Vorkenntnisse) aber nicht von vornherein homolog sind. Da die Homologie jedoch Grundvoraussetzung für die Vergleichbarkeit von Fällen ist, stellt sich die Frage, wie dokumentarisch Forschende mit der typologischen Verschiedenheit von schulischen Lerngruppen umgehen sollen. Der Weg, den Nohl aufzeigt, liegt in der Selektion von Fällen, die auf einer basalen Ebene bereits typische Gemeinsamkeiten aufweisen, und im Vergleichen dieser Fälle auf einer höheren typologischen Bezugsebene.

Im Hinblick auf den Fremdsprachenunterricht könnte die basale Ebene beispielsweise in der Art und Weise der Aufgabenbearbeitung liegen, natürlich immer bezogen auf vergleichbare lernersprachliche Entwicklungsstufen. Liegen hier Gemeinsamkeiten bzw. Homologie vor, so ließe sich im nächsten Schritt z.B. ein Vergleich auf der Ebene von Sprachenfolgen (erste versus zweite versus dritte 
Fremdsprache), Schulformen (Gesamtschule versus Gymnasium), oder Schulstandorten (ländliches versus großstädtisches Einzugsgebiet) anstreben. Die genaue Bestimmung der Relation typischer Ausprägungen von Sprachbewusstheit zu den sozialen Typiken der Interaktion dürfte eine der zentralen Herausforderung zukünftiger rekonstruktiver Fremdsprachenforschung bilden.

\subsubsection{Lernersprache}

Die individuelle Sprachbewusstheit Lernender steht wiederum in direkter Relation zur Ausbildung ihrer Lernersprache. Der Begriff der Lernersprache, im Englischen Interlanguage, geht auf den Linguisten Selinker (1972) zurück ${ }^{15}$. Selinker schreibt im Abstract seines Beitrags Interlanguage:

Dieser Artikel stellt klar, dass wir die für den Prozess des Zweitspracherwerbs relevanten Fakten durch die vergleichende Untersuchung von drei produktiven Sprachsystemen erhalten. Diese sind (1) die Mutttersprache des Lernenden, (2) die fremdsprachliche Kompetenz des Lernenden, seine Zwischensprache (Interlanguage), (3) das System der Fremdsprache.

(Selinker 1972: 31)

Harden (2006: 83) stellt dazu fest: „Weniger die Konkurrenz, und damit die Störungen, Interferenzen und Fehler standen im Vordergrund als vielmehr die Frage nach den Interaktionen von L1, L2 und den kognitiven Aktivitäten der Lerner.“ Mit der ,Entdeckung' der Lernersprache wurde das Tor für eine Sicht auf den Fremdsprachenunterricht geöffnet, die das Lernen und die daran beteiligten Prozesse in den Mittelpunkt rückte.

Parallel zu den erwähnten sozialen Typiken kann und muss bezogen auf den Fremdsprachenunterricht somit noch eine weitere Typik ermittelt werden, die als lernersprachliche Typik bezeichnet werden kann und die an Entwicklungsstände von Performanz und Sprachbewusstheit ${ }^{16}$ gebunden ist (s.o. Kap. 1). Hier

15 obwohl ähnliche Untersuchungen bereits andernorts seit der Mitte der 1960er Jahre unternommen worden waren.

16 Im Kontext der Sprachbewusstheitsforschung werden einige Begriffe verwendet, die sich auch in der rekonstruktiven Fremdsprachenforschung wiederfinden, wie z. B. implizites und explizites Wissen, performatorisches Wissen, deklaratives und prozedurales Wissen. Die terminologische Vielfalt inklusive einiger Parallelbezeichnungen bedarf einer Erklärung. Eichler \& Nold (2007) schreiben dazu: „Der alltagssprachliche Begriff des Sprachgefühls lässt sich aus linguistischer Sicht mit Sprachkompetenz und Kommunikative Kompetenz bezeichnen. Allerdings wird damit in der Linguistik weniger ein Gefühl als vielmehr ein System von sprachlichen Regelungen beschrieben. Aus einer mehr psycholinguistischen und psychologischen Perspektive entsprechen 
greift keine lineare Progression, wie sie sich häufig in Lehrwerken abbildet, eher eine Kumulativität im Sinne des Gemeinsamen europäischen Referenzrahmens für Sprachen (2001), da hier auch unterschiedliche Entwicklungsstände je nach Teilkompetenz mitgedacht werden, z. B. Niveau A2 beim Sprechen und gleichzeitig Niveau B1 beim Lesen. Die fremdsprachlichen Teilkompetenzen entwickeln sich mit unterschiedlichen Geschwindigkeiten, sogar regredierende Lernentwicklungen wurden beobachtet sowie erhebliche geschlechterbedingte, sozial bedingte und migrationsbedingte Disparitäten (s. Köller et al. 2010). Am treffendsten scheint mir daher der Begriff der Kumulativität zu sein (vgl. Bundesministerium für Bildung und Forschung 2003: 18, 26 f.), um den nichtlinearen, individuellen und an die Lernbiografie gekoppelten Charakter des sprachlichen Kompetenzaufbaus zu bezeichnen. Auch das Vygotskysche Modell der zone of proximal development (Vygotsky 1971:236-245) bietet sich hier an, da es zusätzlich noch den Aspekt der sozialen Konstruktion von Wissen (scaffolding, Wood et al. 1976) betont. Die aktuelle, naheliegende und potentielle Entwicklungszone

den Begriffen Sprachgefühl und Sprachwissen in der wissenschaftlichen Diskussion wie bereits erwähnt am ehesten die Bezeichnungen implizites und explizites Wissen. Beim Lernen von Fremdsprachen sind zwei Arten von implizitem Wissen zu berücksichtigen, nämlich unanalysierte sprachliche Versatzstücke und automatisierte sprachliche Regelungen. Beide können von Fremdsprachenlernern ohne ein Wissen über die sprachlichen Regelungen verwendet werden .[...]. Die Begriffe implizites und explizites Wissen werden von anderen Forschern auch mit dem Begriffspaar prozedurales und deklaratives Wissen in Verbindung gebracht und teilweise synonym verwendet [...]“ (Eichler \& Nold 2007: 69).

Zuletzt sei noch die Systematik Gnutzmanns (1997: 232ff.) erwähnt, der im Rückgriff auf James \& Garrett (1991) fünf Domänen von language awareness unterscheidet, eine affektive, eine soziale, eine politische, eine performatorische und eine kognitive Domäne. Neben den sozialen und affektiven Domänen werden besonders die beiden letzteren im Fremdsprachenunterricht relevant:

Performanz-Domäne: „Hier geht es darum, ob und inwieweit Schüler durch Wissen über die Sprache in ihrer sprachlichen Kompetenz und in ihrer Fähigkeit, Grammatik und Wortschatz in Bezug auf ein Kommunikationsziel möglichst effektiv zu verwenden, gefördert werden können“ (zitiert nach Eichler \& Nold: 65).

Kognitive Domäne: „[...] all jene Prozesse, durch die ein Individuum Kenntnis von Gegenständen erhält und durch die es seine Umwelt, also auch seine sprachliche, bewusst wahrnimmt. Es geht also um die geistige Durchdringung des Systems Sprache, somit um das Erkennen von sprachlichen Einheiten, von Kontrasten und Regularitäten auf den verschiedenen sprachlichen Ebenen einschließlich ihrer Funktionen und Verwendungen" (ibd). 
des individuellen lernersprachlichen Sinns (s. Einleitung) können als Kreise visualisiert werden, die mit den anderen Sinnebenen interagieren:

Abbildung 1: Entwicklungszonen des lernersprachlichen Sinns

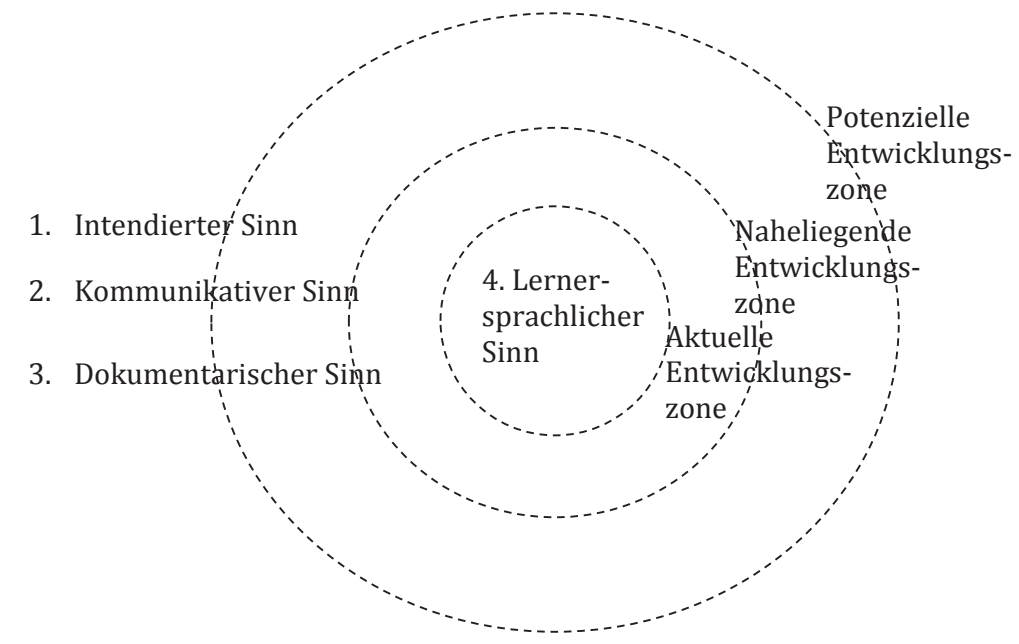

Das Modell in Abbildung 2 fügt dem eher statischen Modell in Tabelle 1 in Form der durch gestrichelte Kreise symbolisierten Entwicklungszonen noch eine dynamische Komponente hinzu. Jenseits dieser eher formalen Sicht ist es jedoch bedeutsam, das Zusammenspiel der beiden Aspekte soziale Sinnkonstruktion und Entwicklungszonen der Lernersprachen zu verstehen. Wenn der lernersprachliche Sinn auch denselben sozialen Konstruktionsprozessen unterliegt wie die anderen Sinnbereiche, so ist doch unverkennbar, dass die unterschiedliche lernersprachliche Performanz wie auch die unterschiedlich entwickelte lernersprachliche Bewusstheit auf die Individualität der Lernenden verweisen. Diese wiederum kann die kollektive Sinnkonstruktion im Fremdsprachenunterricht anregen, aber auch durchkreuzen, ja sogar blockieren. Die Blockade kann bis zur Kommunikationsverweigerung reichen. Die Sinnebenen des Fremdsprachenunterrichts zielen somit heuristisch betrachtet auf die Sozialität des Lernens, während die Entwicklungszonen der Lernersprache eher ihre Individualität abbilden. Diese Dualität zu verstehen, führt uns zu einem tieferen Verständnis des fremdsprachlichen bzw. fremdsprachenunterrichtlichen Lernens. Die unterschiedlichen Entwicklungsstände der Lernersprachen können in der Fremdsprachenforschung zu irritierenden Befunden führen, wenn nämlich 
lernersprachliche Hürden das Textverstehen oder die Textproduktion massiv transformieren. Diese Transformationen können u.U. zu typologisch nur schwer klassifizierbaren Mustern führen (vgl. Kap. 4.1.5).

Die von den sozialen Verhaltensmustern einer Fremdsprachenlerngruppe äußerlich betrachtet scheinbar abgekoppelten individuellen fremdsprachlichen Entwicklungsstände werfen aus methodologischer Sicht die Frage nach der Homologie auf: Sind die fremdsprachlich-performativ und/oder qua Sprachbewusstheit manifestierten Typiken Einzelner mit den sozialen Handlungstypiken einer Klasse überhaupt vergleichbar? Ansätze einer Lösung finden sich, wenn man auf die praxeologischen Ursprünge der Typenbildung in der Dokumentarischen Methode rekurriert, d.h. wenn man berücksichtigt, dass auf allen Handlungsebenen in einer gegebenen Gruppe Wissen nach ähnlichen oder identischen Regeln konstruiert wird. Mit anderen Worten, auch die Konstruktion individuellen fremdsprachlichen Wissens - sei es auf der pragmatisch-performativen Ebene, sei es auf der Ebene bewussten Regelwissens - unterliegt bestimmten Regeln, die rekonstruierbar sind. In der Perspektive ihrer sozialen Konstruiertheit (vgl. Long 1996, Mackey 1999) ist mithin die aus der Linguistik stammende Theorie der Lernersprache anschlussfähig an soziale Interaktionstheorien bzw. Theorien der Sinnkonstruktion im sozialen Miteinander des Fremdsprachenunterrichts (zur sozialen Dimension der Sprachbewusstheit s. James \& Garrett 1991). Das Charakteristikum des Fremdsprachenunterrichts ist ja gerade die Verknüpfung der sozialen Ebenen der Verständigung mit der lernersprachlichen Entwicklung im Lernprozess. Die beiden Ebenen beeinflussen sich wechselseitig (Näheres s. Kap. 2.3). Dies zeigt sich sowohl auf der Mikroebene des Aushandelns sprachlicher Bedeutung als auch auf der Mesoebene der Übernahme und/oder Zuweisung sozialer Rollen. Auf der Mikroebene des Aushandelns sprachlicher Bedeutung sind die sprachlichen Lernhandlungen betroffen, die zu einem Zwischenprodukt führen, z.B. zur Verwendung einer bestimmten lexikalischen oder grammatikalischen Struktur. Diese Lernhandlungsprozesse führen nicht unbedingt zu erfolgreichem Spracherwerb, ja noch nicht einmal unbedingt zu einem korrekten Zwischenprodukt. Es kann zu sprachlich richtigen Zwischenprodukten oder auch zu Fehlern kommen. Wir stoßen hier auf ein offensichtliches Dilemma der fremdsprachlichen Verständigung: Durch grobe Defizite in der individuellen Lernersprache kann es zu Nicht-Verstehen, zu Missverständnissen und in der Folge sogar zum Verstummen der Kommunikation kommen. Sprachliche Defizite können zwar auch die muttersprachliche Verständigung beeinträchtigen, bei der fremdsprachlichen Verständigung besteht das Dilemma jedoch auf unteren Lernniveaus systematisch. 
Psychologisch relevant ist, dass die angestrebte zielsprachliche Verständigung zu elementaren Verunsicherungen führen bzw. die in der Muttersprache erworbenen common grounds der Verständigung wieder in Frage stellen kann. Zielsprachige Kommunikation kann mühsam sein, Sprachnot kann verunsichern, erworbene Konzepte können sich als brüchig erweisen. In der Muttersprache eloquente Redner können in der Fremdsprache stammelnd um Worte ringen. Identitäts- und Rollenkonflikte können die Folge sein. Auf der Mesobene der Übernahme und/oder Zuweisung sozialer Rollen könnte etwa die herausragende performative Leistung eines Schülers im Englischen z.B. nach einem Amerikaaufenthalt seine soziale Isoliertheit unter den Peers bedingen, genauso wie er aber auch als primus inter pares in einer Führungsgruppe hervorragend integriert sein könnte. Auch die fremdsprachlichen Konstruktionsprozesse selbst sind im Unterricht mithin hochgradig sozial beeinflusst: Es kann z.B. zur Unterdrückung eigener sprachlicher Produktion bzw. Konstruktion kommen, um nicht als Streber oder Versager dazustehen.

Das Bindeglied zwischen der Klasse bzw. Lerngruppe als Milieu und der individuellen Sprach(en)- und Sprachlernbewusstheit bildet die individuelle Sprach(en)lernbiografie. Sie kann auf einer soziologischen Ebene als Prägung durch die Migrations- bzw. Sprachgeschichte der Familie, durch die familiale Sprachverwendung sowie durch das kulturelle Kapital des Einzelnen und seiner Familie erklärt werden. Auf die Sprach(en)lernbiografie kann in unterrichtlichen Gruppendiskussionen nur sehr indirekt zurückgeschlossen werden, nur selten finden sich diesbezügliche Verweise. Allenfalls durch gezielte sprachbewusstheitsfördernde Aufgabenstellungen werden mitunter sprach(en)biografische Hintergründe im Fremdsprachenunterricht direkt sichtbar. Sie können jedoch mit Hilfe des narrativen biografischen Interviews rekonstruiert werden (Trautmann 2015; s. auch Kap. 2.4 und 3.2), das damit vorzüglich als Triangulationsinstrument zur Analyse des Unterrichtsgeschehens eingesetzt werden kann.

Aus dem beobachteten individuellen Sprachlernverhalten und der beobachteten individuellen Sprachbewusstheit heraus lassen sich durch Fallvergleich neben der sozialen Typik somit auch an Sprachbewusstheit orientierte Typiken generieren, z.B. der an hoher mündlich-kommunikativer Performanz orientierte Typus, der an hoher formalsprachlicher Korrektheit orientierte Typus, der theoretisierende bzw. analytische Typus oder der Typus des praktisch orientierten Anwenders. 


\subsubsection{Lerngruppenmilieus und konjunktive Erfahrungsräume}

Kommen wir nun noch einmal zur Frage zurück, was für die alltägliche Verständigung im Fremdsprachenunterricht charakteristisch ist. Zur Beantwortung dieser Frage erweisen sich die Verfahren der Dokumentarischen Methode und der Ethnomethodologie als hilfreich. Die Ethnomethodologie wie im Übrigen auch fast zeitgleich die Vertreter der Grounded Theory (Glaser \& Strauss 1967) waren als eine Methodenkritik an der scheinbar allmächtigen Messpraxis der damaligen Sozialwissenschaften aufgetreten. Nun stellt sich Sozialität im schulischen Fremdsprachenunterricht äußerlich betrachtet als eine hoch ritualisierte Form der Kommunikation dar. Es werden wie bei - von den Ethnomethodologen untersuchten - bürokratischen Organisationen häufig ritualisierte Anweisungen gegeben und in ebenso ritualisierter Form ausgeführt. Es werden häufig bestimmte Fragetypen eingesetzt, um den Unterrichtenden meist bereits bekannte Antworten zu erzielen. Eine Konvention dieser Rituale im Kontext des Unterrichts lautet: Unterrichtete folgen (als Laien) dem Wissen der Unterrichtenden (Experten). Doch welche Konsequenzen leiten sich aus dieser und anderen Konventionen der Verständigung im Unterricht ab? Schüler interpretieren die Äußerungen der Unterrichtenden wie auch die der Mitschüler vor dem Hintergrund ihres kollektiven, geteilten Wissens. Zu diesem Wissen ist mit standardisierten Verfahren wie z.B. Fragebögen nur schwer vorzudringen. Es ist ein Wissen, das aus gemeinsamer Praxis entstanden, habitualisiert und vorbewusst ist. Es ist jedoch, wie oben erläutert, rekonstruierbar, und zwar über die Art und Weise seiner Entstehung. Um beim obigen Beispiel „Esteban“ aus dem Spanischunterricht zu bleiben: Nicht allein das Faktum der Korrektur einer lernersprachlichen Äußerung - das Was - interessiert, sondern das Wie dieser Korrektur. Im genannten Beispiel zeigt sich z.B. die Konfrontation mit einem negativen Gegenhorizont, der Drohung mit dem Ausstieg aus der gemeinsamen Rahmung. Das Wie führt zu der dahinter liegenden (gespielten?) Rivalität der beiden Mädchen. Wir wohnen einem habitualisierten Korrekturverhalten bei, das im Fremdsprachenunterricht als Typus verfestigt scheint und seinen Ursprung in dem milieutypischen Lernhandlungsverhalten in Bildungseinrichtungen und dort wiederum im engeren Sinne in dem milieutypischen Lernhandlungsverhalten bestimmter verwandter Unterrichtsfächer findet. Es handelt sich nicht um ein Großmilieu im Sinne von Bourdieu (1982) oder Gurwitsch (1976), sondern um ein Milieu auf Grundlage gruppenhafter Erfahrungen. Das milieutypische Lernhandlungsverhalten hängt jedoch auch von der Klassenkomposition ab: Handelt es sich um eine sozialräumlich eher homogene Klasse (z.B. dominiert von Jugendlichen mit einem bestimmten Migrationshintergrund, eventuell aus 
einem bestimmten Kiez, von Jugendlichen aus einem ländlichen Einzugsgebiet oder aus einem kleinstädtischen Einzugsgebiet)? Handelt es sich um eine stark leistungsheterogene Klasse (z.B. mit einem hohen Anteil an Inklusionskindern, Kindern mit Leserechtschreibschwäche, etc.)? Hat die Klasse einen hohen Anteil Akademikerkinder, die ihr kulturelles Kapital besser ausspielen können als Kinder aus Elternhäusern ohne akademischen Bildungshintergrund? Wie ist die Geschlechterverteilung? Ist die Klasse entwicklungshomogen oder entwicklungsheterogen zusammengesetzt (z. B. einige noch sehr kindliche Lernende neben bereits stark pubertierenden Lernenden)? Auch die Lehrpersonen formen das milieutypische Lernhandlungsverhalten mit. Geschlechterspezifik und Generationenspezifik spielen z.B. eine Rolle. Wie ,nahe` steht die Lehrperson den Lernenden auf Grund ihrer generationalen Erfahrungen? Verschmilzt sie fast mit ihnen auf Grund des geringen Altersabstands oder trennt sie eine Kluft von vierzig Lebensjahren? Welche Rolle spielen Innovationsbedürfnis und Routinen bei ihr? Wie stark sind bestimmte Lehrkonzepte habitualisiert (vgl. Tesch 2010).

Aus den Merkmalsaufzählungen wird deutlich, dass mit der Bezeichnung „milieutypisches Lernverhalten einer Klasse oder Lerngruppe“ ein spezifisches Milieuverständnis zugrunde gelegt wird. In ihm wird eine Klasse oder Lerngruppe im soziologischen Sinne als (Mikro-)Milieu begriffen, das von Schülerinnen und Schülern sowie von den Lehrpersonen gleichermaßen geprägt wird. Sie kommen über längere Zeiträume (ein, zwei oder mehr Jahre) im festen institutionellen Rahmen regelmäßig zusammen, um ritualisierten Tätigkeiten nachzugehen. Sie teilen somit wissenssoziologisch betrachtet einen ganz bestimmten „konjunktiven Erfahrungsraum“ bzw. „Erlebniszusammenhang“ (s. Mannheim 1980), der rekonstruierbar ist und der die sprachlichen Aushandlungsprozesse in einer Klasse, Lerngruppe oder Kleingruppe prägt. Verfügt der Forscher über eine hinreichende Anzahl vergleichbarer Fälle, so lässt sich daraus eine Typik generieren. Dies kann nach Bohnsack (2014: 51f.) eine Entwicklungstypik, eine Bildungsmilieutypik, eine Geschlechtstypik, eine Generationstypik und eine Typik des sozialräumlichen Milieus sein. Hinzu kommt - mit Blick auf die Besonderheiten der Institution Schule - u. a. eine Organisationstypik sowie eine Medientypik. Auf Grund der schulpolitischen und sozialen Entwicklungen der letzten Jahre wäre man geneigt, auch eine Migrations-, eine Inklusionstypik sowie - der Gegebenheit Rechnung tragend, dass es auch soziale Zusammenhänge gibt, die gerade erst im Entstehen sind und als begrifflich etablierte Formen der Kollektivität noch gar nicht zur Verfügung stehen - eine „relationale Typik“ (vgl. Nohl 2013) hinzuzufügen. Gegen eine solche „relationale“ Typenbildung ließe sich einwenden, dass eine Relation selbst nur schwerlich einen Typus bilden kann, da sich 
dieser eigentlich auf einen abgrenzbaren Gegenstand (Geschlecht, Generation, Bildungsmilieu etc.) und darauf gerichtete abgrenzbare Orientierungsrahmen beziehen müsste. Mehr noch: Die Abgrenzbarkeit der Orientierungsrahmen ist geradezu das Bestimmungsmerkmal eines Typus (Bohnsack 2014: 11; Nohl 2013: 49). Dafür spricht jedoch, dass dynamische bzw. im Entstehen begriffene gesellschaftliche Phänomene wie Migration oder Inklusion durchaus konkrete Bezüge oder Bezugsgruppen aufweisen, deren Erfahrungsräume gleichwohl erst in ihren Relationen zu anderen Typiken wie Geschlecht, Bildungsmilieu oder Organisation rekonstruierbar sind. (Näheres zur Typenbildung s. Kap. 3.1.5).

Bonnet (2007: 225) diskutiert die Annahme eines konjunktiven Erfahrungsraums in Schulkassen und wendet ein, dass Klassen möglicherweise in Subgruppen bzw. disparate Erfahrungsräume zerfallen können, die „ein intensives interaktives Einpendeln auf gemeinsame Begriffe" (ibd.) nicht mehr zulassen. Tatsächlich unterscheidet sich die konjunktive Erfahrung im Klassenzimmer von vielen bisher rekonstruktiv erforschten Milieus wie etwa den Jugendlichen in einer fränkischen Kleinstadt (Mangold \& Bohnsack 1988) durch den hohen Grad institutioneller Umrahmung und die hoch ritualisierten und vor allem aufgabengesteuerten lernintentionalen Abläufe im Klassenzimmer. Dennoch ist zu vermuten, dass die gemeinsamen Erfahrungen in Klassen, die oft über mehrere Jahre zusammen sind, ein ähnliches generationales Erleben aufweisen, wie andere Gemeinschaften auch (z.B. Familie, Militär, Berufsgruppe). Bei Kindern und Jugendlichen beansprucht die Schule und insbesondere ihre Klasse meist den gesamten Alltag, d.h. sie verbringen mehr Zeit in der Klassen- und Schulgemeinschaft als mit ihrer Familie. Die große generationale Zusammengehörigkeit von Schulklassen zeigt sich daher mitunter noch bei späteren Klassentreffen.

Bonnet (2007: 224 f.) stellt das Vorhandensein konjunktiver Erfahrungsräume in Schulklassen nicht generell in Frage, sondern konstatiert, dass das SichEntziehen von Individuen im Extremfall zu schwindender Konjunktivität führen kann, zu bloßen „Anwesenheitsräumen“:

Für den jeweils untersuchten Fachunterricht kann man somit nicht von vornherein unterstellen, dass der unterrichtliche Erfahrungsraum für die Orientierungsrahmen der Lernenden überhaupt relevant wird - dass er also im eigentlichen Sinne überhaupt ein Erfahrungs- und nicht bloß ein Anwesenheitsraum ist. Analytisch liegt es nahe, beim Begriff des Erfahrungsraums zu bleiben, sehr wohl kann aber dessen Konjunktivität nicht vorausgesetzt werden, sondern muss sich in der Analyse überhaupt erst erweisen. Gegenstand der Dokumentarischen Analyse ist es dann nicht, die konjunktive Kraft des Erfahrungsraums in Form eines kollektiven Orientierungsrahmens lediglich aufzufinden. Vielmehr geht es darum, den Erfahrungsraum und die ihn strukturierenden Orientierungen überhaupt zu rekonstruieren und seine Konjunktivität oder Disparativität 
festzustellen, sowie in letzterem Fall herauszuarbeiten, in welche tatsächlichen Erfahrungräume der Anwesenheitsraum Unterricht zerfällt" (ibd).

Das Ermitteln von Konjunktivität und Disparitivität im Unterricht impliziert, dass neben der „Suche nach metaphorisch dichten Stellen [auch die Suche] nach Brechungen, Leerstellen, Widersprüchen oder Mehrdeutigkeiten interessant [wird]“ (Bonnet 2012: 229). Hinzuzufügen wäre, dass auch der Diskursverlauf im Fachunterricht auf Grund der starken Steuerung durch die Aufgabenstellungen eine Selbstläufigkeit der Diskursentwicklung nur selten zulässt; ein Wiederaufgreifen bestimmter Themen ist ebenfalls selten und kommt wenn überhaupt nur in den Flüstergesprächen und Parallelinteraktionen (vgl. ibd.: 225 und Tesch 2010: 124) zum Vorschein. Das Augenmerk des Unterrichtsforschers muss somit sowohl diesen Flüstergesprächen und Parallelinteraktionen als auch den ,klassenöffentlichen' Aushandlungen gelten. Sie aufeinander zu beziehen und dabei Indexikalitäten, Widersprüche und Inkongruenzen herauszuarbeiten, ist die Herausforderung der Datenanalyse von Unterrichtsaufzeichnungen. Leerstellen, Schweigepausen, scheinbare Abwesenheiten bekommen dann ihre eigentliche Bedeutung. Sie verweisen auf die Parallelexistenz von Erfahrungsräumen, deren Konjunktivität sich oft auf der Ebene von Subgruppen und nicht im Klassenplenum entfaltet. Zu Recht bemerkt Bonnet (2007: 225), dass sich damit auch die Suche nach Fokussierungsmetaphern ${ }^{17}$ verändert. Tatsächlich sind es im Unterricht nicht immer die Stellen hoher interaktiver bzw. metaphorischer Dichte, sondern auch vielfach ritualisierte versus spontane Äußerungen, die Tonlage (s.o. Passage „Esteban“), ein ritualisiertes Kichern, nonverbale Kommunikation wie Mimik oder die Platzierung im Klassenzimmer und generell auch die scheinbare Nichtbeteiligung, die ,stumme Sprache', die unser forschendes Interesse auf sich ziehen. Wiederum zurecht schließt Bonnet daher auch auf eine notwendige Modifikation im Hinblick auf die Verwendung des Begriff des „Orientierungsrahmes“: Neben der kollektiven Orientierung in der Großgruppe oder der Subgruppen erkennen wir im schulischen Unterricht auch Individualorientierungen. Diese spiegeln sich beispielsweise in individueller Orientierung an Progression oder in den per se individuellen Selbstwirksamkeitserwartungen. Hier schließt sich der Kreis zu der bereits in Kapitel 2.1.6 konstatierten Dualität von Sozialität und Individualität im Fremdsprachenunterricht.

17 dramaturgische Höhepunkte in der Diskursentwicklung, häufig im Modus metaphorischer Formulierungen 


\subsubsection{Ein sozial-interaktionales Lehr-Lernmodell des Fremdsprachenunterrichts}

Abschließend soll nun die Frage gestellt werden, welches Lehr-Lernmodell der wissenssoziologischen Perspektive auf der Ebene der Sinnkonstruktion im Fremdsprachenunterricht entsprechen könnte. In den Fremdsprachendidaktiken war es lange Zeit eher unüblich, ein eigenes Lehr-Lernmodell zu Grunde zu legen. Man überließ diese Aufgabe der Erziehungswissenschaft und orientierte sich implizit an Theorien des individuellen Wissensaufbaus. Die bis in die 1980er Jahre verbreitetste Theorie war die des progressiven linearen und stufenweisen Wissensaufbaus vom Einfachen zum Komplexen, meist orientiert an der Systemgrammatik. Sie wurde vor allem von den Lehrwerken transportiert, wobei die Verlage meist mit den ,Bedürfnissen' der Basis (vor allem der Lehrenden) argumentierten, d.h. mit der Nachfrage der Kunden. Die empirische Frage nach der Sozialität des Lernens wurde der normativen Forderung nach sozialem Lernen und der methodischen Frage seiner Ausgestaltung (kooperatives Lernen) untergeordnet. Dies änderte sich mit der Rezeption des Konstruktivismus (Wolff 2002), allerdings bremste das ,Hereinbrechen' der Kompetenz- und Standardorientierung nach PISA 2000 die gerade angebrochene konstruktivistische Ära in der deutschen Fremdsprachendidaktik wieder. Auch erste praxeologische Ansätze (z.B. Börner 2000; Eckerth 2003) wurden zunächst von der politischen Forderung überlagert, vom Output oder Outcome her zu denken. Doch könnte durch die Verbindung des praxeologischen Ansatzes mit der Kompetenzorientierung auch in der Fremdsprachenforschung der Blick wieder mehr für das Unterrichtsgeschehen geöffnet werden (z.B. Bonnet 2009, 2012; Tesch 2010; Steininger 2014; Bracker 2015).

Welche Bezugspunkte für ein wissenssoziologisch basiertes fremdsprachenunterrichtliches Lehr-Lernmodell liegen nun vor? Sowohl die Rekonstruktion der sozialen Sinnebene als auch die der individuellen lernersprachlichen Sinnebene verweisen auf eine Basisannahme, die mit der Sozialisationstheorie der objektiven Hermeneutik verwandt und auch an die Lerntheorie Vygotskys anschlussfähig ist:

- Die Lernenden lernen gerade dadurch, dass sie von den Lehrenden „so behandelt werden, als ob sie kompetent seien" (Bohnsack 2014: 74).

Lehrende stimulieren bei einer bestimmten Unterrichtsgestaltung gelingendes Lernen, indem sie den Lernenden die benötigten, Gerüste‘ zur Verfügung stellen (scaffolding). Sie stellen damit eine positive Kompetenzerwartung her, indem sie Lernenden ,zumuten', ein höheres Niveau, eine nahe liegende Entwicklungszone 
auch tatsächlich zu erreichen, auch wenn dies performatorisch (noch) nicht gelingt. Scheiterndes Lernen hingegen wird induziert, wenn Lehrpersonen ein Gelingen erwarten, ohne dass gleichzeitig die benötigten Gerüste bereit stehen oder bereit gestellt werden. Diese Basisannahme für gelingenden Unterricht lässt sich auf der Grundlage von Unterrichtsanalysen empirisch fundieren (s. Tesch 2010), wobei eine genauere Bestimmung der Beziehung von Individualität und Sozialität im Fremdsprachenunterricht noch aussteht.

Ein wissenssoziologisch begründetes unterrichtliches Lehr-Lernmodell beruht weiterhin auf der Annahme, dass - wie in Kapitel 2.1.2 bis 2.1.7 ausführlich dargestellt - Lernen auf individuellen und kollektiven Sinnkonstruktionen beruht:

- Sinn konstituiert sich im (Fremdsprachen-)Unterricht auf verschiedenen Ebenen und wird interaktiv ausgehandelt.

Diese zweite Basisannahme kann wiederum ergänzt werden durch eine dritte Basisannahme:

- Kommunikation unterliegt (auch) im (Fremdsprachen-)Unterricht einem Bedürfnis nach Erlebniskohärenz, d.h. nach kooperativ gestalteten, für alle verständlichen, thematisch relevanten und im Ablauf möglichst störungsfreien Interaktionsprozessen.

Diese Basisannahme lässt sich durch kommunikationstheoretische bzw. konversationsanalytische Forschungen stützen. Kallmeyer \& Schütze (1976) stellen im Anschluss an Schütz (1974) Prinzipien und Aktivitäten der Kooperation heraus. Sie gelten auch für den schulischen Unterricht:

Diese Aktivitäten folgen grundlegenden Interaktionsprinzipien, die als Interaktionspostulate, interpretative Prozeduren bzw. Basisregeln der Kommunikation bezeichnet werden. Derartige Basisregeln beinhalten die Bedingungen der Möglichkeit von Kommunikation. Kern dieser Bedingungen ist die Bewältigung der grundsätzlichen Unvereinbarkeiten des Interaktionsprozesses: die Unvergleichbarkeit der beteiligten Selbstidentitäten, die Vagheit der ausgetauschten Symbolgesten, die mangelnde Angleichung ihrer wechselseitigen Interpretation usw. Zur Bewältigung dieser Probleme bilden die Beteiligten sogenannte praktische Idealisierungen.

(Kallmeyer \& Schütze 1976: 9)

Unter dieser Annahme unterstellen sich die Kommunikationspartner gegenseitig, Gesprächsgegenstände so auszuwählen und Gesprächsregeln so anzuwenden, dass die Kommunikation gelingt. Die Bezeichnung "praktische Idealisierungen“ entspricht auf der Ebene des oben skizzierten Lehr-Lernmodells dem konstruktivistischen Ideal gelingenden Lernens, bei dem Lehrende Lernenden prinzipiell 
Kompetenz ,unterstellen' und die notwendigen Gerüste bereitstellen, damit die Lernenden die nächste Entwicklungszone erreichen können (s. o). Kallmeyer \& Schütze (ibd.) nennen die wichtigsten Prinzipien für gelingende Kommunikation. Ich wende sie im Folgenden auf den Fremdsprachenunterricht an:

\section{Kooperativität}

Dieses Prinzip bedeutet im Fremdsprachenunterricht die Bereitschaft, alternierend die Sprecher- und die Zuhörerrolle einzunehmen, sich auf Regeln des Sprecherwechsels zu verständigen und diese Regeln auch tatsächlich einzuhalten.

2. Verstehen

Das Verstehen wird im Fremdsprachenunterricht häufig durch lernersprachliche Inkongruenzen erschwert, meist fehlendes Vokabular, aber auch durch unterschiedliche Weltwissensbestände. Somit besteht eine der Hauptaufgaben zur Herstellung von Verstehen im Fremdsprachenunterricht darin, diese Inkongruenzen zunächst zu diagnostizieren und anschließend das notwendige Sprach- und Referenzwissen bereitzustellen bzw. zu seinem Erwerb anzuregen.

3. Ablaufkonstitution

Aufbau, Sicherung und Beendigung eines Gesprächsablaufs setzen auch im Fremdsprachenunterricht voraus, dass grundlegend die Gesprächsbereitschaft signalisiert und durch eine sozial angemessene Verteilung des Rederechts organisiert wird. Sodann gilt es, die Themen so zu wählen, dass ausreichende Relevanz für alle Beteiligten gesichert ist. Um die Kohärenz zu sichern, sind Themen- oder Fokuswechsel innerhalb eines gesetzten Themas zu signalisieren. Schließlich sind Störungen zu verarbeiten oder abzuwehren. Diese Prinzipien dienen dazu, das Bedürfnis nach Erlebniskohärenz zu befriedigen. Eine Unterrichtssequenz wie die oben dargestellte Übung zur Personenbeschreibung („Esteban“) wird von den Lernenden als geschlossene Episode erlebt. Sie dient zwar intentional dem Zweck, einen bestimmten Lernschritt zu vollziehen, besitzt jedoch auch eine Struktur als soziale Gesprächseinheit. Diese in kooperativer Grundhaltung, für alle verständlich, thematisch relevant und im Ablauf möglichst störungsfrei zu gestalten, wird idealerweise als Gemeinschaftsaufgabe wahrgenommen (vgl. Bohnack 2014: 95). Ihr Gelingen wie auch ihr Nichtgelingen können zum Gegenstand rekonstruktiver Unterrichtsforschung gemacht werden.

Zusammenfassend wurden in diesem Kapitel die wissenssoziologischen, linguistischen und lerntheoretischen Bezüge der rekonstruktiven Fremdsprachenforschung mit der Dokumentarischen Methode vorgestellt. Den Kern des Kapitels 
bildet der analytische Zugang zur Klassenzimmerinteraktion auf zwei Ebenen, nämlich auf der sozialen und auf der lernersprachlichen Ebene, wobei gezeigt wurde, dass es sich hierbei nicht um parallele Phänomene handelt, sondern um interaktiv verwobene. Auch die Lernersprache kann demzufolge und im Anschluss an die soziokulturelle Theorie des Lernens als sozial konstruierte analysiert werden. Daraus ergibt sich aus forschungsmethodischer Sicht eine doppelte Interpretationsleistung, allerdings auch ein unbestreitbarer theoretischer Mehrwert. Dieser Mehrwert ist auf den verschiedenen Ebenen der Sinnkonstruktion im Fremdsprachenunterricht sichtbar zu machen, was Gegenstand der nun drei folgenden Kapitel zur Textanalyse (Verständigung durch Texte, Kapitel 2.2), zur Aufgabenanalyse (Verständigung über Texte, Kapitel 2.3) und zur Interviewanalyse (Verständigung über erlebten Unterricht sowie über das Lehren und Lernen, Kapitel 2.4) sein wird. In diesen drei Kapiteln sollen die zentralen Ebenen der Verständigung herausgearbeitet und dargestellt werden, auf die die rekonstruktive Fremdsprachenforschung mit der Dokumentarischen Methode sinnvoll bezogen werden kann.

\subsection{Verständigung durch Texte}

Jeglicher Text, sei es Schrift-, Bild- oder Tontext, impliziert einen Textsinn, der durch sein bloßes Vorhandensein eine Verständigung konstituiert, sofern ein Textrezipient existiert. Die Analyse von Texten im Hinblick auf ihren immanenten und exmanenten Sinn, d.h. die Bestimmung ihres Inhalts und ihrer Wirkung (z.B. Stilanalyse) gehören zum Grundbestand der Sprachendidaktik, sei es in der Erstsprache oder in anderen Sprachen. Verschiedene $-z$. T. ältere - hermeneutische Traditionen wie z.B. New Criticism (Ransom 1941; Wellek 1978; Wimsatt \& Beardsley 1954), Rezeptionsästhetik (Iser 1972; Jaus 1970), Erzähltheorie (z.B. Genette, 1998), Didaktik des Fremdverstehens (Bredella \& Christ 1995), Literacies-Ansätze (Kern 2000; The New London Group 2000) lassen sich im schulischen Lernkontext bis hin zu aktuellen Abiturprüfungsaufgaben fachdidaktisch transformiert vorfinden und in der Fremdsprachenforschung als Deutungsmuster rekonstruieren. Es ist hier nicht der Ort, auf diese Verfahren näher einzugehen; eine Anmerkung freilich soll gemacht werden: In der Didaktik des Fremdverstehens ging es u. a. um den Perspektivenwechsel als notwendige Voraussetzung für interkulturelles Verstehen und die damit einhergehende Veränderung bei Lernenden bzw. generell bei fremdsprachlich Handelnden. Durch die dem Perspektivenwechsel vorausgehende Perspektivenwahrnehmung und Perspektivendifferenzierung rekonstruiert der Leser eine dem Text eingeschriebene Sinnstruktur. Aus meiner Sicht bietet die rekonstruktive 
Fremdsprachenforschung mit der Dokumentarischen Methode in der Analyse von Verständigung durch Texte in Verbindung mit der Analyse der Verständigung über Texte eine bisher noch wenig genutzte empirische Grundlage zur Erforschung des Fremdverstehens im Unterricht. Im Folgenden wird an einigen Beispielen gezeigt, auf welchen methodologischen Grundlagen die Untersuchung der Verständigung durch Texte aus rekonstruktiver Perspektive erfolgen kann. Ich beginne mit der Verständigung durch literarische Texte und beschließe das Kapitel mit Ausführungen zur Verständigung durch Bildtexte.

\subsubsection{Verständigung durch literarische Texte}

In literarischen Texten findet eine Betrachtung der Welt aus bestimmten Blickwinkeln statt, z.B. dem Blickwinkel bestimmter Protagonisten, dem Blickwinkel eines auktorialen Erzählers oder dem scheinbar objektiven Blickwinkel einer fiktiven Kamera. Es wird erzählt von Abläufen oder von Handlungen in der Zeit, von Orten, von menschlichen Beziehungen etc., und es wird berichtet von Gefühlen, Einsichten, Überlegungen, von Rationalem und Irrationalem. Lesen bedeutet, all diese Berichte, Erzählungen und Darstellungen zu rekonstruieren. Der Sinn wird hier nicht im Diskurs kollektiv konstruiert, wie etwa in einer Gruppendiskussion. Er ist dem Text vielmehr bereits - z. B. als Autorenrolle, als Leserrolle, als innere Logik oder als Brüche - eingelassen. Dies bedeutet jedoch nicht, dass keine gemeinsame Sinnkonstruktion stattfindet. Sie ist vielmehr verlagert bzw. verschoben in die Text-Leser-Interaktion (vgl. Bredella 2007).

In seinem Aufsatz „Fremdverstehen und Bildung durch neue Weltansichten: Perspektivenvielfalt, Perspektivenwechsel und Perspektivenübernahme durch Literatur“ (2007) geht Nünning auf die Aspekte ein, die mit der „Verständigung durch literarische Texte“ gemeint sind. Er nennt aus rezeptionsästhetischer Sicht vier Ebenen des Fremdverstehens beim Umgang mit literarischen Texten:

- „Fremdverstehen im Sinne des sprachlichen Verstehens eines literarischen Textes, also die Dimension des Textverstehens (vgl. Grzesik 1990),

- Fremdverstehen im Sinne des Verstehens eines fremdsprachlichen literarischen Textes, also die fremdsprachige Verstehensdimension,

- Fremdverstehen im Sinne des Verstehens einer fremden Kultur bzw. ihrer Darstellung in einem fremdkulturellen literarischen Text, also die ,fremdkulturelle Verstehensdimension' (Funke 1990: 592),

- Fremdverstehen im Sinne des Einfühlens in bzw. Verstehens von fiktionalen Figuren aus einer fremden Kultur, also eine komplexe Mischung von interpersonalen, literarischen und fremdkulturellen Verstehensprozessen."

(Ibd.: 126) 
Am folgenden Beispiel eines literarischen Textauszugs soll gezeigt werden, wie rekonstruktives Textverstehen mit der rezeptionsästhetischen Sicht verbunden werden kann.

Rafael Chirbes Roman En la orilla (2013) (am Ufer) fängt folgendermaßen an:

26 de diciembre de 2010

El primero en ver la carroña es Ahmed Ouallahi.

Desde que Esteban cerró la carpintería hace más de un mes, Ahmed pasea todas las mañanas por la Marina. Su amigo Rachid lo lleva en el coche hasta el restaurante en que trabaja como pinche de cocina, y Ahmed camina desde alli hasta el rincón del pantano donde planta la caña y echa la red. Le gusta pescar en el marjal, lejos de los mirones y de los guardias. Cuando cierran la cocina del restaurante - a las tres y media de la tarde -, Rachid lo busca y, sentados en el suelo a la sombra de las cañas, comen sobre un mantel tendido en la hierba. Los une la amistad, pero también se brindan un servicio mutuo. Pagan a medias la gasolina del viejo Ford Mondeo de Rachid, una ganga que consiguió por menos de mil euros y ha resultado ser una ruina porque, según dice, traga gasolina con la misma avidez con que un alemán bebe cerveza.

26. Dezember 2010

Der erste, der den Kadaver sieht, ist Ahmed Ouallahi.

Seit Esteban die Schreinerei vor einem Monat geschlossen hat, geht Ahmed jeden Morgen auf der Uferpromenade spazieren. Sein Freund Rachid nimmt ihn im Wagen bis zum Lokal mit, in dem er als Küchengehilfe arbeitet, und von dort spaziert Ahmed bis zu der Ecke des Stausees, wo er die Angelrute einsteckt und das Netz auswirft. Er angelt gerne an der sumpfigen Stelle, weit weg von neugierigen Blicken und von Polizisten. Wenn die Küche des Lokals schließt - nachmittags um halb vier - kommt Rachid zu ihm, und beide essen dann auf dem Boden sitzend, im Schatten der Weidenrohre, auf einer auf dem Gras ausgebreiteten Tischdecke. Beide verbindet eine Freundschaft, aber sie tun sich auch gegenseitig einen Gefallen. Sie teilen sich das Benzin für Rachids alten Ford Mondeo, ein Schnäppchen, das er für unter tausend Euro gekauft hatte und das sich dann als Fass ohne Boden herausstellte, weil der Wagen, wie es heißt, genauso gierig Benzin schluckt wie ein Deutscher Bier trinkt. (Übers. BT)

Auf der Ebene des kommunikativen (immanenten) Textsinns lässt sich folgender Inhalt wiedergeben. Am 26. Dezember 2010 sieht Ahmed eine Leiche (Tier oder Mensch). Ahmed geht jeden Morgen an der Uferpromenade spazieren, wo ihn sein Freund Rachid mit dem Wagen abholt und zum Restaurant mitnimmt, in welchem Rachid als Küchengehilfe arbeitet. Von dort geht Ahmed zu Fuß zum Stausee, um zu angeln. Nachmittags nach der Arbeit kommt auch Rachid dorthin. Die Freunde teilen sich das Benzin für Rachids alten Ford Mondeo.

Immer noch auf der kommunikativen (immanenten) Sinnebene können wir nun die ersten drei der vier von Nünning genannten Dimensionen anwenden: 
a) Dimension des Textverstehens: Die oben zusammengefasste Handlung leitet einen literarischen Erzähltext ein, der zur Gattung des Romans gehört. Der Autor nennt einen konkreten Zeitpunkt und einen noch unkonkreten Ort ( $e l$ pantano). Drei Personen werden genannt, von denen zwei (Ahmed und Rachid) direkt handeln und einer (Esteban) indirekt. Von Esteban erfahren wir, dass er eine Schreinerei besitzt oder besaß, die er vor einem Monat geschlossen hat. Seitdem geht Ahmed jeden Morgen spazieren. Es ist daher naheliegend, dass Ahmed in Estebans Schreinerei beschäftigt war. Wir vermuten, dass die beiden finanzielle Schwierigkeiten haben, daher teilen sie sich das Benzin für Rachids Auto.

Erzählerisch bedeutsam sind die kriminalistischen Hinweise in den ersten beiden Zeilen: eine präzise Datumsangabe und die Aussage, dass Ahmed als erster eine Leiche entdeckt. Damit wird eine Spannung bzw. Leseerwartung hinsichtlich der Aufklärung der Identität der Leiche aufgebaut. Im Moment ist noch offen, ob es sich um einen Tierkadaver oder um eine menschliche Leiche handelt.

Die Erzählzeit ist das Präsens, was zum direkten Miterleben der geschilderten Situation einläd.

b) Verstehen eines fremdsprachigen literarischen Textes: Der Textauszug ist auf der grammatisch-syntaktischen Ebene für ein mittleres fremdsprachiges Niveau (B1) gut zu verstehen, enthält jedoch sehr präzise Lexik, die nur mit Hilfe eines Wörterbuchs selbstständig erschlossen werden kann.

c) Fremdkulturelle Verstehensdimension: Die beiden Protagonisten tragen nicht-europäische Namen; es handelt sich vermutlich um nordafrikanische Arbeitsmigranten im spanischen Mittelmeerraum. Spätere Textstellen verweisen auf die Wirtschaftskrise in Spanien.

Auf der vierten Ebene d), der Ebene des Fremdverstehens im Sinne des Einfühlens in bzw. Verstehens von fiktionalen Figuren aus einer fremden Kultur tritt nun auch der dokumentarische Sinn hervor: Durch die präzise und unpathetische Erzählweise über die Freundschaft und gleichzeitig die prekäre Existenzgrundlage Ahmeds und Rachids kann sich gegebenenfalls ein Perspektivenwechsel und eine Empathie für die beiden entfalten. Diese Ebene der Verständigung ist die am schwersten erschließbare; sie liegt nicht offen zutage, könnte jedoch aus seitens des Lesers über Lese-Gespräche oder Lautdenk-Protokolle erschlossen werden. Im Fremdsprachenunterricht können zu diesem Zweck die vom Autor eingesetzten narrativen Merkmale des Textes analysiert werden. Dazu zählen der Spannungsaufbau, die Einführung weiterer Personen und Schauplätze, die Entwicklung der Erzählsituation, auch die sprachlich-stilistischen Mittel (Bilder, 
sprachliche Register, sprachlicher Code, etc.). Am Ende kann es gelingen, die „komplexe Mischung von interpersonalen, literarischen und fremdkulturellen“ (ibd.) Textaspekten zu rekonstruieren, das heißt, die Eigenart des Erzählstils und die besondere Wirkung, die von ihm ausgeht.

Auf allen vier Ebenen greift die in Kap. 2.1 erwähnte Indexikalität in der Verständigung: Der Autor verständigt sich mit dem Leser durch Verweise auf gemeinsame Wissensbasen: Textwissen (Ebene a), sprachliches Wissen (Ebene b), fremdkulturelles Wissen (Ebene c), Perspektivenwechsel und Empathiefähigkeit (Ebene d). Der Leser ist eingeladen, dem Autor auf seiner, Führung' durch eine fremde Welt zu folgen; der Autor verspricht dem Leser im Gegenzug eine zwar nicht faktisch-authentische aber in ihrer Fiktionalität nicht minder reichhaltige neue Welterfahrung.

Gerade dieser Wesenszug fiktionaler Texte, die Nicht-Faktizität, das Gestaltetsein, begründet ihren dokumentarischen Sinn. Rekonstruktive Fremdsprachenforschung ist daran interessiert, die spezifische Gestaltung fiktionaler Texte offenzulegen. Dabei interessieren nicht die echten Personen, die dem Autor vielleicht als Vorlage seines Romans gedient haben mögen, sondern die Figuren Ahmed, Rachid und Esteban. Die narrative Gestaltung ihrer Geschichte (und der vielen anderen Personen, die im Roman noch auftauchen werden) interessiert, nicht die Tatsache, dass es vielleicht einen realen Ahmed, einen realen Rachid und einen realen Esteban gibt oder gegeben hat.

Soweit besteht Parallelität zwischen dem literaturdidaktischen Vorgehen, wie es sich beispielsweise in moderneren Lehrwerken des Fremdsprachenunterrichts spiegelt, und dem Anspruch rekonstruktiver Analysen. Diese gehen indess noch über die Ebene einzelner narrativer Merkmale hinaus. Um an die Unterscheidung der Kunsthistoriker Panofsky (1975) und Imdahl (1994) zwischen Ikonografie und Ikonologie bzw. Ikonik anzuknüpfen: Der Erkenntnisgewinn rekonstruktiver Analysen liegt im Wechsel vom Narrativen zum Narratologischen bzw. zur Narrativik. Dieser Wechsel wird möglich durch den für die Dokumentarische Methode typischen Wechsel der Analyseeinstellung vom Was der Erzählung zu ihrem Wie. Dieser Wechsel der Analyseeinstellung ist sowohl auf den Text als auch auf den Textproduzenten anzuwenden. Auf der Textebene gilt es, die Eigengesetzlichkeit bzw. die Eigensinnigkeit des Textes herauszuarbeiten, was es erforderlich macht, vom textlichen und sprachlichen Vorwissen der Rezipienten abzusehen und den Text als selbstreferentielles (autopoietisches) System zu verstehen (s. Bohnsack 2014: 163). Dies erlaubt es dem Interpreten, zum spezifischen Erfahrungsraum des Textes in Gestalt eines bestimmten, dem Text eigenen (z.B. narrativen) Habitus vorzudringen. Auf diesen von Mannheim (1980: 88) als 
„Einklammerung des Geltungsbereichs“ bezeichneten Akt werde ich im folgenden Abschnitt zur Bildinterpretation noch näher eingehen.

Auf der Ebene der Textproduzenten wiederum könnte der Text En la orilla durch Textvergleiche als gattungstypisch oder epochetypisch bezeichnet werden. In dem Maße, wie es dem Forscher gelingt, den Habitus der abgebildeten Personen vom Habitus der abbildenden Person (s. Bohnsack 2014: 162) zu unterscheiden, entsteht somit eine Narratologie, z.B. eine Theorie des spanischen Krisenromans seit 2010, dessen Hauptfigur Rafael Chirbes geworden ist. Parallelitäten zu literaturtheoretischen Ansätzen des Poststrukturalismus sowie zur Habitustheorie Bourdieus (1982) sind offensichtlich, was im sehr fortgeschrittenen Fremdsprachenunterricht der gymnasialen Oberstufe Anwendungsmöglichkeit für literarisches Textverstehen bietet (s. Burwitz-Melzer 2007).

\subsubsection{Verständigung durch Bildtexte}

Bohnsack (2014: 158 f.) hebt den Umstand hervor, dass die Verständigung durch das Bild ganz im Gegensatz zur Verständigung über das Bild bisher einen völlig vernachlässigten Bereich sozialwissenschaftlicher Forschung darstellt. Dabei sind

Bilder auf einer ganz fundamentalen Ebene der Verständigung und des Lernens, der Sozialisation und der Bildung (auch außerhalb der Massenmedien) Medium alltäglicher Verständigung und alltäglichen Handelns.

(Ibd. 159)

Er führt in diesem Zusammenhang die Bedeutung „,innerer Bilder“ aus, d.h. der ikonischen Schemata, die für unsere Deutung äußerer Bilder, ja für die Deutung der Symbolik von Sprache ganz generell einen wesentlichen Anteil haben. Unsere „inneren Bilder“ sind die im Laufe unseres Lebens verinnerlichten „vorreflexiven“ (ibd.) Wissensbestände über „soziale Szenerien, Gebärden, Gestik und Mimik“ (ibd. 159 f.). Damit bringt er auf den Punkt, worin das Besondere des Bildes liegt, das nämlich, was nur der Bildtext zu leisten vermag im Gegensatz zum Schrifttext. Dieses vorreflexive sprachunabhängige Wissen, die „inneren Bilder", gilt es, mit Hilfe der Dokumentarischen Methode und dem Wechsel der Analyseeinstellung vom Was zum Wie herauszuarbeiten.

Die folgenden zwei Beispiele sollen verdeutlichen, wie auch bei der Bildanalyse der Wechsel der Analyseeinstellung möglich bzw. erforderlich ist, um die formale Ganzheitsstruktur eines Bildes zu erkennen. Ferner wird deutlich, wie die Bildanalyse auf der Tiefenstruktur insbesondere im Fremdsprachenunterricht unerlässlich ist, um den Anspruch an interkulturelles Lernen erfolgreich einzulösen. 
a) Das Pressefoto Cérémonie d'accueil d'étrangers accédant à la nationalité française (Empfangszeremonie für Ausländer anlässlich der Überreichung der französischen Staatsangehörigkeit) von Georges Bartoli/Divergence wurde im Cahier von LE MONDE am 16. November 2013 veröffentlicht.

b) Das Werbephoto eines französischen Kulturkaufhauses aus dem Jahre 2005 stammt aus dem Internet.

Abbildung 2: LE MONDE, 16. November 2013, cérémonie d'accueil, Copyright George Bartoli/Divergence

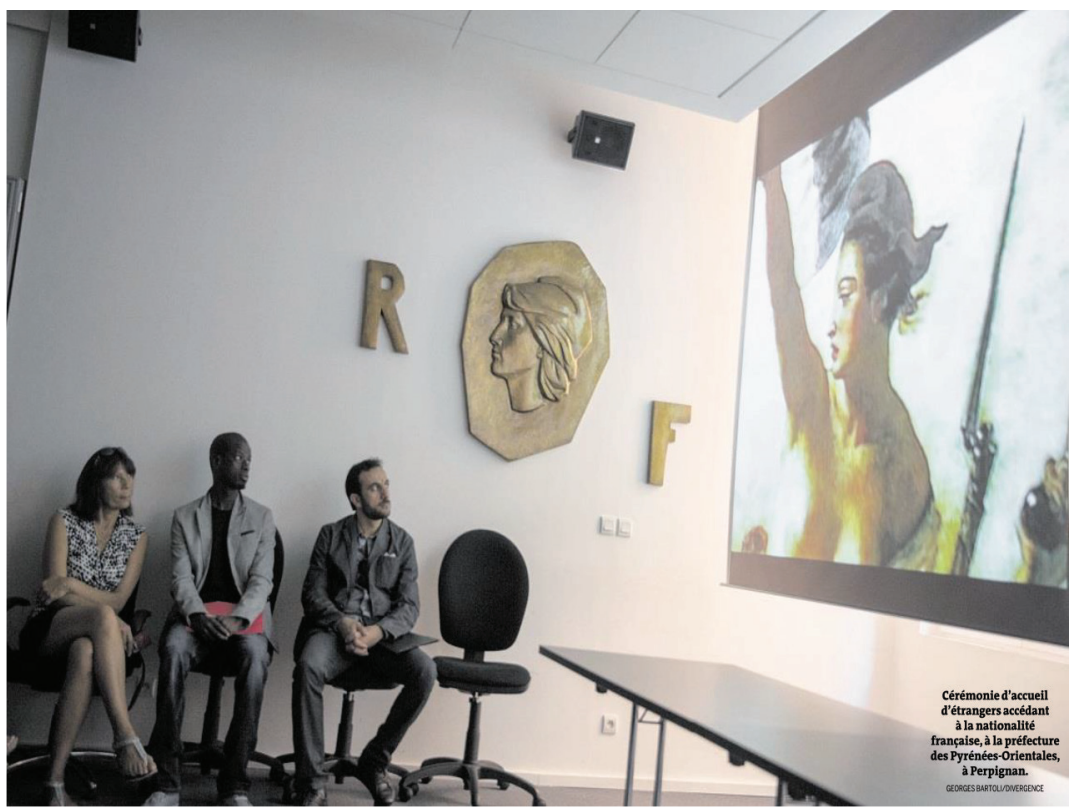

Eine Bildanalyse könnte zunächst die kommunikative Sinnebene herausarbeiten, in die das gesamte Vorwissen des Betrachters einfließt, sein Kontextwissen (z.B. Bedingungen der Erlangung der französischen Staatsbürgerschaft, staatstragende Prinzipien der französischen Republik, Identifikation des Ortes - eine Präfektur, daher auch die beiden Initialen R und F für République française, Identifikation der Marianne aus dem Gemälde von Delacroix La Liberté guidant le peuple, etc.). Genau dieses Vorwissen jedoch verhindert das Bildverstehen auf der Ebene der „inneren Bilder“, d.h. „,soziale Szenerien, Gebärden, Gestik und Mimik“ (ibd.). Würde man nicht zu dieser Ebene vordringen, so würde das Pressefoto von 2013 keinerlei eigene Aussage jenseits derjenigen besitzen, einen bestimmten sozialen 
und politischen Kontext zu illustrieren. Um zu dieser ureigenen Wirkung des Bildes vorzudringen, zur Evokation innerer Bilder, ist es erforderlich, das Vorwissen sozusagen zu suspendieren. Dieser Vorgang wird in der Dokumentarischen Bildinterpretation die „Einklammerung des ikonografischen bzw. konnotativen Sinngehalts“ (ibd.: 164) oder nach Mannheim auch „Einklammerung des Geltungscharakters" (Mannheim 1980: 88) genannt. Gleichzeitig erfolgt damit eine Einklammerung des Geltungsanspruchs auf normative Richtigkeit. Es wird ausgeblendet, was das Bild alles bedeutet, bedeuten könnte oder bedeuten soll außerhalb seines ureigenen inneren Gestaltetseins.

Um dieses innere Gestaltetsein zu erschließen, bedarf es wie erwähnt des Wechsels der Analyseeinstellung, das heißt des Wechsels zur formalen Bildkomposition. Bohnsack lehnt sich in dieser Hinsicht an Imdahl (1996) an, der drei Dimensionen der formalen Bildkomposition differenziert:

a) Die perspektivische Projektion:

Durch die Wahl der Perspektivität wird in der je bildspezifischen Art und Weise Räumlichkeit und Körperlichkeit konstruiert. Es wird damit eine Gesetzmäßigkeit in das Bild hineingetragen, die im wahrsten Sinne des Wortes Einblicke in die Perspektive des abbildenden Bildproduzenten und in seine Weltanschauung, seinen Habitus vermittelt.

b) Die szenische Choreografie des Bildes

Bei Imdahl [...] ist diese definiert als, die szenische Konstellation der in bestimmter Weise handelnden oder sich verhaltenden Figuren in ihrem Verhältnis zueinander, also als deren soziale Bezogenheit. Dies betrifft die räumliche Positionierung der Akteure bzw. Figuren zueinander ebenso wie den Bezug ihrer Gebärden, aber auch ihrer Blicke, aufeinander.

c) Die planimetrische Ganzheitsstruktur des Bildes:

Diese bezieht sich auf die Strukturen des Bildes in der Ebene oder Fläche. [...]. Die beiden anderen Dimensionen, die ,perspektivische Projektion und die szenische Choreografie erfordern ein wiedererkennendes, auf die gegenständliche Außenwelt bezogenes Sehen (...). Dagegen geht die planimetrische Komposition, insofern sie bildbezogen ist, nicht von der vorgegebenen Außenwelt, sondern vom Bildfeld aus, welches sie selbst setzt'.

(Bohnsack 2014: 168).

Am Bildbeispiel Cérémonie d'accueil d'étrangers accédant à la nationalité française lässt sich leicht erkennen, dass der Photograph das Bild der Marianne als Fluchtpunkt zweier zentraler Achsen wählte, durch die die abgebildeten Personen einem Arrangement unterworfen werden. 


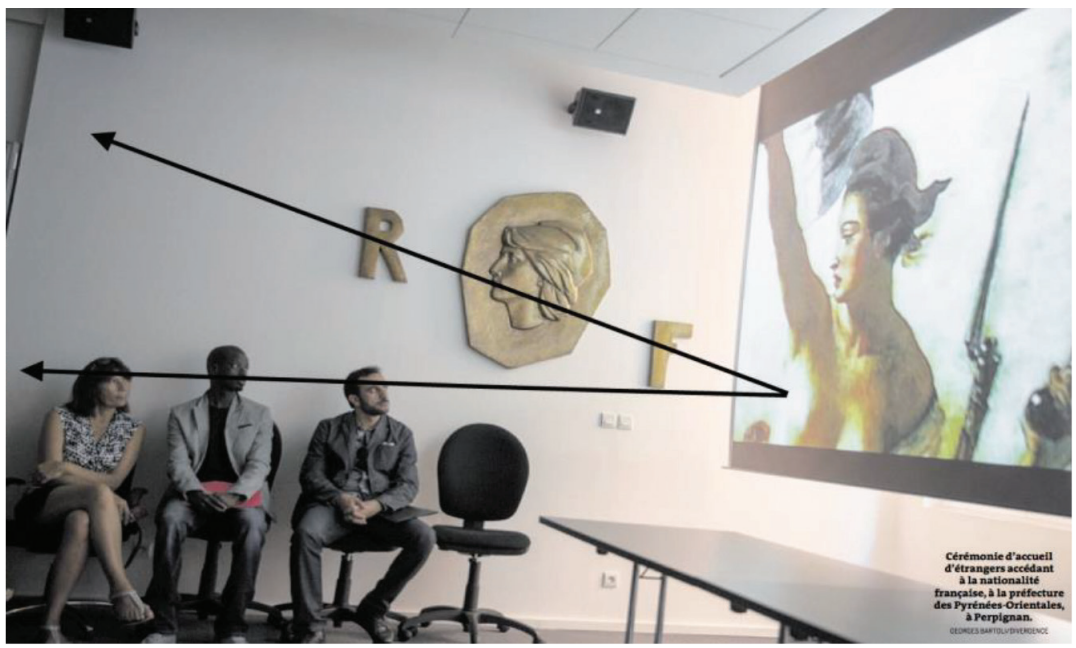

Auf der Ebene der szenischen Choreografie blicken die drei Personen von unten nach oben auf das Abbild der Marianne, die wiederum gleichsam von oben nach unten auf sie herabschaut. Auch die République Française gehört zum ,Oben: Die Gebärdensprache unterstreicht den Gegensatz: Hier der kämpferische fahnenschwenkende Gestus der Marianne mit einem (Gesichts-)Ausdruck kühler Unerbittlichkeit, dort die skeptischen Blicke der drei Einwanderer, die sich in Reserviertheit hüllen, Unterwerfung suggerierend. Die planimetrische Ganzheitsstruktur des Bildes schließlich offenbart, dass sich der Gesamtausschnitt in der Schräge befindet. Der Photograph hat die Kamera vor der Aufnahme leicht gedreht, wodurch die Personen einerseits wie aus dem Gleichgewicht geraten erscheinen und eine Art Instabilität erzeugt wird. Das Ganze ist sozusagen ins Rutschen gekommen und könnte noch mehr in Schieflage geraten (zur Bildinterpretation s. auch Tesch 2015).

Eine erweiterte Zugangsmöglichkeit zum Wesenskern des Bildes über die Analyse der Perspektivik, der Planimetrik und der Bildkomposition hinaus wäre der Bildvergleich. Er erlaubt es, der isolierten Einzelbetrachtung weitere empirische Vergleichshorizonte gegenüberzustellen und damit das Epoche- und Kulturtypische eines Bildes zu erfassen. Wenn wir den Ausschnitt aus Delacroix La Liberté guidant le peuple, das bereits im Photo der cérémonie d'accueil eine Rolle spielte, einmal öffnen und das ganze Bild betrachten, tritt ein Bild zutage, das durch die Komposition ausgewählter Elemente und Personen, durch die 
Achsenbildung, durch Zentrierung sowie Vorder- und Hintergrundbildung Pathos vermittelt:

Abbildung 4: Eugène Delacroix, 1830, La Liberté guidant le peuple, Fluchtachsen

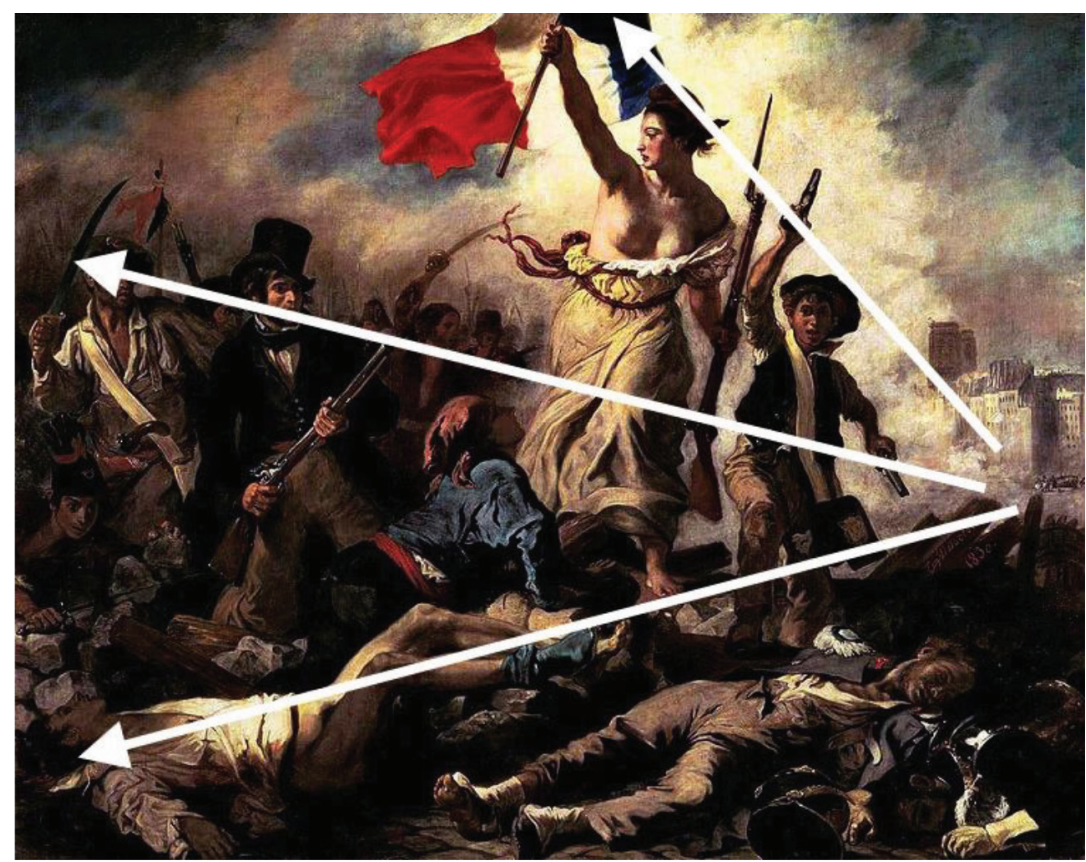

Im Zentrum des Bildes steht die Freiheit, die das Volk zum Sieg über die Reaktion führt. Letztere liegt vernichtet am Boden, während, das Volk' im Kampfesnebel bereits über sie hinwegschreitet.

Eine ganz andere Dramaturgie wählt dagegen Georges Bartoli bei seinem Photo der cérémonie d'accueil: Hier soll gerade kein Pathos vermittelt werden, sondern eine Spannung zwischen den Ansprüchen der République, die in ihrer kühlen Strenge die universelle Geltung der von ihr verkörperten Werte symbolisiert, und den skeptisch-schüchternen Blicken der neu Eingebürgerten, welcher Herkunft oder Hautfarbe sie auch seien. Die Dramaturgie vermittelt hier nicht Aufbruch, nicht den messianischen Kampf für eine bessere Zukunft oder die Rettung der Welt, sondern die kühle Sicherung des Erworbenen, Statik statt Dynamik. Es genügt offensichtlich, die Marianne mit einem Beamer zu zeigen: Ihr Blick und das Bajonett beherrschen die Szene. Verstärkt wird diese Dramaturgie durch die leichte Neigung des gesamten Bildes: Menschen müssen sich der Macht 
von Recht und Gesetz beugen, Integration ist Pflicht. Die Analyse mit Hilfe der Dokumentarischen Methode zeigt, wie der dokumentarische Bildsinn durch ein Offenlegen der formalen Bildkomposition herausgearbeitet werden kann.

Das Werbephoto des französischen Kulturkaufhauses FNAC aus dem Jahre 2005 benutzt eine konträre Bildkomposition ${ }^{18}$. Der Blick fällt zunächst auf den mittig angeordneten Kaufhausnamen „fnac“, der in großen weißen Lettern von innen beleuchtet auf einem violetten Hintergrund prangt. Als nächstes nimmt der Betrachter die geschlossenen und vergitterten Eingangstüren wahr. Es handelt sich um zwei identische Portale mit zum Halboval gerundeten Griffbügeln, die bis zum Boden reichen. Erst jetzt bemerkt der Betrachter, dass in der linken Ecke des Photos neben dem linken Portal noch ein kleines buntes Kuppelzelt steht. Es suggeriert, dass dort jemand die Nacht verbringt, um beim Öffnen der Portale am nächsten Morgen als erster ins Kaufhaus hineinzukommen.

Der Blick wandert nun nach oben, wo in weißen Lettern auf schwarzem Grund zu lesen ist „N’attendez plus ... “ (Warten Sie nicht länger) und darunter kleingedruckt „Faites vos achats $24 \mathrm{~h} / 24,7 \mathrm{j} / 7$ et téléchargez vos musiques préférées sur $\rightarrow w w w$.fnac.com" (Machen Sie Ihre Einkäufe 24 Stunden am Tag, sieben Tage in der Woche und laden Sie ihre Lieblingsmusik bei www.fnac.com herunter). Die Internetadresse ist als einziger Schriftzug in schwarzen Lettern auf weißem Grund gestaltet. In der obersten Zeile steht in Großbuchstaben aber kleinerer Schriftgröße ,livres - musiques - DVD \& vidéo - image \& son - logiciels \& jeux - micro \& télécom - voyages -spectacles“. In der unteren rechten Ecke ist in sehr kleiner Schriftgröße zu lesen „certifié non conforme“ (zertifiziert nicht konform), dann folgt fnac.com mit dem Schriftzug fnac in das offizielle Logo eingekleidet.

Es gibt nur eine ganz schwach ausgebildete Perspektivik, die durch das in die linke Ecke gedrängte kleine Kuppelzelt erzeugt wird. Das Bild wird dominiert durch den Schriftzug fnac, d.h. man kann von einer klaren Zentrik sprechen. Auch die szenische Choreografie ist weniger ausgeprägt als auf dem Pressefoto von 2013 und wird lediglich durch das in die Ecke gedrängte kleine Zelt hergestellt. Fast übersieht man es, so stark ist die Wirkung des zentralen Blocks, der aus den beiden geschlossenen Doppeltüren und dem leuchtenden Schriftzug gebildet wird. Zur szenischen Choreografie gehört allerdings auch die völlige Abwesenheit von Personen, die absolute Statik und Stille, die Verschlossenheit der vergitterten Tore und die Wahl der Nacht als Zeit-Raum der Aufnahme: Es

18 Auf Grund fehlender Abdruckrechte kann das Photo selbst hier nicht veröffentlicht werden. 
ist das Bild einer machtvollen Festung, einer Art Fort Knox, das einen Schatz beherbergen muss. In der planimetrischen Ganzheitsstruktur enthüllt sich das Geheimnis der Wirkung, denn über dem Bild leuchtet ein noch mächtigerer Schatz, das Internet. Zelt und verschlossene Türen wirken dadurch plötzlich nicht mehr zeitgemäß, ein paar zarte Buchstaben verraten, wo die Zukunft liegt: 24h/24, 7j/7. Und wer Französisch versteht, errät: Nattendez plus ... (Warten Sie nicht länger ...).

Alle vorgestellten Bilder können im Französischunterricht sehr gut als Impulse für Interpretationsgespräche eingesetzt werden, die von der Analyse der Bildkomposition ausgehen.

In diesem Kapitel wurde gezeigt, wie mit Hilfe des Wechsel der Analyseeinstellung vom Was zum Wie der einem Text - sei es ein Schrifttext oder Bildtext - eingelassene Sinn herausgearbeitet werden und damit auch die spezifische Wirkung künstlerisch gestalteter Texte analysiert werden kann. In der Regel des Fremdsprachenunterrichts wird diese Analyseleistung von Experten erbracht, die etwa als Redaktionsteams eines Lehrwerks den Textsinn in einen Aufgabensinn transformieren. Dazu bedarf es zwar keiner expliziten Kennntis der Dokumentarischen Methode. Die Ausführungen zeigen jedoch, dass Textverstehen als Rekonstruktion von Textsinn kongruent sein kann zu bestimmten Analyseschritten der Dokumentarischen Methode und dass diese somit als ein allgemeines heuristisches Verfahren der Fremdsprachendidaktik und Fremdsprachenforschung genutzt werden kann.

Im folgenden Kapitel wird aufgezeigt, wie die Transformation des didaktischen Sinns einer Aufgabe beschrieben und seine weitere Transformation in Lernendensinn analysiert werden kann.

\subsection{Verständigung über Texte}

Die Verständigung über Texte (in der Regel durch Aufgaben) bildet den zentralen Anwendungsbereich der Fremdsprachenforschung mit der Dokumentarischen Methode, da hier der Unterricht selbst, also der Entstehungsprozess sprachlicher und kultureller Konstruktionen, beobachtet und auf dieser Basis die Regelhaftigkeit kommunikativen Handelns im Unterricht erforscht werden kann. In Schulklassen oder längerfristig konstituierten schulischen Lerngruppen besitzen Schülerinnen und Schüler zusammen mit ihrer jeweiligen Lehrperson, wie bereits in den vorangehenden Kapiteln ausgeführt, „konjunktive Erfahrungsräume“ im Sinne Mannheims (1980), da sie auf sozial geteiltes Hintergrundwissen zurückgreifen. Sie können sich dieses geteilte Hintergrundwissen jedoch selbst nicht nach Belieben bewusst machen. 
Die Funktion des Kapitels 2.3 besteht darin, an Hand zweier Beispiele die Rekonstruktion der Aufgabenbearbeitung zu illustrieren. Rekonstruktiv Forschende rekonstruieren zunächst den Aufgabensinn. Das Beispiel zum Französischunterricht aus Tesch (2010: 288 f.) verdeutlicht das Gemeinte. Es geht in Kapitel 2.3.1 um die Rekonstruktion eines Bild-Text-Sinns (Werbefoto FNAC). Ergänzend wird in Kapitel 2.3.2 das bereits erwähnte Beispiel zum Spanischunterricht dargeboten (vgl. Kap. 2.1, Personenbeschreibung Esteban), in dem die Konstruktion lernersprachlichen Sinns im Vordergrund steht.

Aus psycholinguistischer Sicht (Levelt 1989) impliziert die Aufgabe, eine Bild- oder Personenbeschreibung durchzuführen, die Fähigkeit, abgebildete Personenmerkmale zu konzeptualisieren, sie sodann mit Hilfe eines bestimmten Wortschatzes sowie grammatikalischen Wissens zu formulieren, sie anschließend zu artikulieren und korrekt zu vozieren. Hinzu kommt noch ein umfassendes Selbstmonitoring bezogen auf eventuelle Kommunikationsstörungen (Fehler und Fehlerkorrektur). Aus rekonstruktiv-praxeologischer Sicht geht es darum, die sozial-interaktionalen Umstände der Aufgabenbearbeitung herauszuarbeiten und ggfs. zu den psycholinguistischen Prozessen, soweit sie performativ zu Tage treten, in Bezug zu setzen.

\subsubsection{Bild-Text-Aufgabe}

Der Aufgabensinn besteht aus dem Textpotential und dem Aufgabenpotential. Beide Komponenten werden im Folgenden als erstes vorgestellt. Danach erfolgt eine Analyse der beobachteten Aufgabenbearbeitung.

Beispielaufgabe FNAC, 4. Lernjahr Französisch (s. Tesch 2010: 288 f.)

\section{Textpotential}

In der Französischstunde in einer zehnten Klasse zu einer Werbeidee eines großen französischen Kulturkaufhauses wurde das im vorangehenden Kapitel 2.2 vorgestellte Werbefoto besprochen. Das Textpotential des Werbephotos der FNAC wurde oben (Kap. 2.2) bereits mit Blick auf die immanenten Strukturmerkmale herausgearbeitet. Aus fachdidaktischer Sicht könnte das Textpotential noch durch interkulturelle Bezüge ergänzt werden, die sich etwa aus einem internationalen Vergleich von Mediennutzungsgewohnheiten und von Internetkaufverhalten im Besonderen ergeben. Dazu könnten die Lernenden selbst befragt werden bzw. Auskunft erteilen. Darüber hinaus wäre es wünschenswert, entsprechende Zusatzinformationen (z.B. statistische Angaben) zu recherchieren. Auch die Frage, inwieweit kulturelle Produkte wie Konzerte oder Musik-CDs lediglich als Ware oder auch als Daseinsaspekt jenseits des reinen 
Warenwerts betrachtet werden können, ist als Textpotential implizit im Werbefoto der FNAC enthalten. Damit kann der Text durchaus als potentiell passend zu den thematischen Interessen von Fünfzehnjährigen eingeschätzt werden, die als Zielgruppe vorgesehen waren.

\section{Aufgabensinn (intendierter Sinn)}

Um das Aufgabenpotential einzuschätzen, bedarf es - über das Textpotential hinausgehend - einer Analyse der Aufgabenstellung und der individuellen Lernvoraussetzungen. Das ursprüngliche Aufgabenkonzept, das in drei Klassen erprobt wurde, sah vor, das Foto als eine Übung für die Diagnose des Leseverstehens und für die Vermittlung kulturellen Wissens (Angebotsspektrum der FNAC) zu nutzen. Die Aufgabenstellung lautete (Tesch et al. 2008, CD-ROM):

Abbildung 5: Arbeitsblatt der Lernaufgabe Pir@tes du net (Copyright IQB 2008)

Ce document est
$\quad \square$ une publicité pour un magasin.
$\quad \square$ une page d'un site sur Internet.
$\quad \square$ une invitation au voyage.
$\quad \square$ une affiche de cinéma.
Répondez aux questions. Citez le texte.
1. Qu'est-ce qu'on peut faire à la FNAC ? (1)
2. Quelle est l'adresse du site Internet de la FNAC? (1)
3. Qu'est-ce qu'on peut faire sur ce site? (2)

Zwei der im Unterricht beobachteten Lehrkräfte hielten sich an dieses Konzept, wodurch das Bildpotential außen vor blieb und lediglich der Schrifttext genutzt wurde. Die dritte Lehrkraft nutzte das Foto jedoch abweichend von der vorgegebenen Aufgabenstellung als stummen Impuls zur Initiation eines Unterrichtsgesprächs, in dem die Schüler erste Vermutungen zum Inhalt und zur Intention äußern konnten. Diese substantielle Modifikation des Aufgabekonzepts sollte eine zusätzliche Lerngelegenheit für das zielsprachliche Sprechen bieten. Insbesondere das Rätselraten um das Zelt und um die Vertriebsstruktur der FNAC gab zu zahlreichen Spekulationen Anlass (s. u.). Dennoch bleibt auch die modifizierte 
Aufgabenstellung eine Sprachübung. Ihre kommunikative Reichweite ist noch relativ eingeschränkt, es gibt noch keinen Arbeitsplan im Sinn einer komplexen Aufgabe bzw. task (Ellis 2003).

Eine andere Aufgabenvariante zur Nutzung des Textpotentials könnte darin bestehen, zunächst nur den reinen Bildteil des Fotos ohne Textleisten zu zeigen, zu beschreiben und zu interpretieren und erst nach erfolgter Hypothesenbildung das komplette Werbefoto. Der Vorteil eines solchen gestuften Vorgehens könnte darin bestehen, die Wirkung der doppelten Kodierung (Bild plus Text) besser zu verstehen. Die Frage stellt sich, ob diese Verstehensleistung innerhalb der jeweiligen individuellen Entwicklungszonen der Lernenden liegt; die Antwort ist in der Praxis der Aufgabenbearbeitung zu suchen.

\section{Aufgabenbearbeitung}

Die Aufgabenbearbeitung in der Klasse, in der das Bild zunächst als stummer Impuls eingesetzt wurde, führte zu folgender Interaktion zwischen Catrin, Ulf, Sophia, einer nicht identifizierten Stimme (SS) sowie der Lehrerin Frau Reichenbach (s. Tesch 2010: 288).

\footnotetext{
Catrin Ähm il y a peut-être un magasin de tente parce que là il y a un tente.

Reich. De dehors le magasin. D'accord

Ulf Ähm je pense que le magasin a qc äh a qc pour acheter äh bon marché qc que qn äh qc que ähm jeder

Reich. Chaque personne

Ulf Chaque personne veut avoir et les personnes qui dort äh dans le tente ähm veut devenir (.) nein veut ähm (.) veut être les premières personnes qui peut acheter ça

Reich. Exactement. Mhm très bien alors vous vous avez une idée hein déjà Saskia

Saskia Je pense que ähm c'est un magasin ähm qui (.) ähm qui qui va a ouvert ähm ähm äh le matin et le personne ähm qui dort ähm dans la tente veut avoir ça que ähm le ma nee le magasin ähm ähm (.) a qc sans äh kostenlos

SS@@(.)@

Reich. Gratuit (S lachen) gratuit gratuit Aha@(.)@Et-

Saskia $\quad \mathrm{L}$ Et la personne veut avoir ça

Reich. Aha soit gratuit soit bon marché. C'est comme Ulf a dit.

Sophia Peut-être c'est un magasin ähm ou ähm on peut achet acheter des vêtements et c'est le temps de Sommerschlussverkauf

Reich. Les soldes [Schritte: geht zur Tafel, schreibt Begriff an Tafel] d'accord ce sont les soldes quand qc est très très bon marché d'accord hein hein (3) Regardez

[lange Pause: $25 \mathrm{~s}$ ]

Ulf il a déjà parlé de la page d’accueil (.) Oui (.) Vous la voyez aussi maintenant ? On la de on la voit même deux fois. Hm. (3) Ulf
} 
(051 - 077; Auf eine Übersetzung wird an dieser Stelle verzichtet, da sie die lernersprachlichen Merkmale der Interaktion ausblenden würde)

Es zeigt sich in der Aufgabenbearbeitung, dass die formale Struktur, die szenische Choreographie, performativ nicht zum Ausdruck kommt, sondern stattdessen sofort versucht wird, das Angebot des Kulturkaufhauses zu ermitteln. Die spezifischen Gestaltungselemente des Werbefotos und damit seine manipulative Wirkung zu erkennen, wird nicht erwartet bzw. liegt noch außerhalb der Verstehensmöglichkeiten der Lernenden, jedenfalls ohne entsprechendes scaffolding. Ein solches scaffolding wäre z. B. durch die oben angedeutete Trennung von Bildund Textinformation in zwei Bearbeitungsphasen gegeben.

Die gruppenhafte Rahmung liegt somit nicht im kritischen Verstehen der Bildkomposition, sondern in den beiden Themen ,Kulturkaufhaus' und ,Internetkauf‘. Diese Rahmung ist in dieser Phase relativ stark, d.h. die Lernenden empfinden das Thema als relevant für sie. Die Relevanz wird jedoch an keiner Stelle explizit, sie bleibt latent und stimuliert die Aufgabenbearbeitung.

Die Lernersprache entwickelt sich durch soziale Mediation, d.h. unter anderem durch kooperative sprachliche Konstruktionsprozesse mit Peers und Lehrpersonen, wie sich an folgendem Ausschnitt aus obiger Unterrichtsepisode zeigt:

Ulf Ähm je pense que le magasin a qc äh a qc pour acheter äh bon marché qc que qn äh qc que ähm jeder

Reich. Chaque personne

Ulf Chaque personne veut avoir et les personnes qui dort äh dans le tente ähm veut devenir.

Ulf übernimmt die Wortgruppe chaque personne (jeder) durch die Mediation Frau Reichenbachs. Frau Reichenbach bietet ihm chaque personne als scaffold des Typs ,Übersetzung' an. Damit ist eine lexikalische Expansion der Lernersprache auf der performativen Ebene verbunden, die es Ulf ermöglicht, die Verständigung flüssig aufrechtzuerhalten und die Episode zusammen mit Sophia und Frau Reichenbach „erlebniskohärent“ (s. Kap. 2.1.8) zu konkludieren. Alle drei Prinzipien für gelingende Kommunikation sind in dieser Episode realisiert. Allerdings findet keine Reflexion des eigenen Sprachverhaltens im Sinne von expliziter Sprachbewusstheit statt. 


\subsubsection{Aufgabe Personenbeschreibung}

\section{Beispielaufgabe Esteban, 2. Lernjahr Spanisch}

\section{Textpotential}

Es handelt sich um einen Textauszug aus dem Lehrbuch ;Apúntate! (Cornelsen 2008: 89). Der Text ist didaktisiert, d.h. ausschließlich für Lehr-Lernzwecke konstruiert:

Abbildung 6: Textauszug aus ¡Apúntate! (Cornelsen 2008: 89)

\section{Esteban}

Esteban es activo y un poco travieso. Sus actividades favoritas son el fútbol y el voleibol. Por la noche la familia cena y después Esteban a veces chatea con sus primos de Costa Rica.

Sus asignaturas favoritas son Educación Física y Tecnología, pero también Ciencias de la Naturaleza y Geografía e Historia. Esteban sólo tiene un problema: ¡Está harto de Mates!

\section{Esteban}

Esteban ist aktiv und ein wenig trotzig. Seine Lieblingsbeschäftigungen sind Fußball und Volleyball. Am späten Abend isst die Familie zusammen, und manchmal chattet Esteban mit seinen Cousins aus Costa Rica.

Seine Lieblingsfächer sind Sport und Technik, aber auch Naturwissenschaften und Erdkunde/Geschichte. Esteban hat nur ein einziges Problem: Er hat die Nase voll von Mathe. (Übers. BT)

Das Textverstehen entspricht sprachlich betrachtet dem Niveau A2 des gemeinsamen europäischen Referenzrahmens für Sprachen (Europarat 2001: 76): „Kann aus einfacheren schriftlichen Materialien wie Briefen, Broschüren oder Zeitungsartikeln, in denen Ereignisse beschrieben werden, spezifische Informationen herausfinden“.

Es ist dem Text überdies kulturelles Wissen eingelagert:

- über Zeiten und Gewohnheiten (das Abendessen wird por la noche - nachts, bei Dunkelheit - eingenommen),

- über Familienbande zwischen Kontinenten (sus primos de Costa Rica, seine Cousins aus Costa Rica) und

- über spezifische Fächergruppierungen (Ciencias de la Naturaleza y Geografía e Historia, Naturwissenschaft und Geografie-Geschichte: anders als in Deutschland üblich jeweils ein Fach). 


\section{Aufgabensinn (intendierter Sinn)}

Es soll einerseits ein Text verstanden und andererseits auch ein neuer Text produziert werden: Lisa soll die Person Esteban mit Hilfe eines Arbeitsblatts mündlich beschreiben.

Verwendetes Arbeitsblatt (Auszug):

Abbildung 7: Arbeitsblatt zu ¡Apúntate! (Cornelsen 2008: 89)

\begin{tabular}{|c|c|}
\hline Así son los chicos & \\
\hline $\begin{array}{l}\text { nombre: } \\
\text { ¿cómo es?: } \\
\text { asignatura favorita: } \\
\text { también es bueno/-a en: } \\
\text { no es bueno/-a en: } \\
\text { actividades después de las clases: }\end{array}$ & Elena \\
\hline $\begin{array}{l}\text { nombre: } \\
\text { ¿cómo es?: } \\
\text { asignatura favorita: } \\
\text { también es bueno/-a en: } \\
\text { no es bueno/-a en: } \\
\text { actividades después de las clases: }\end{array}$ & Esteban \\
\hline
\end{tabular}

\begin{tabular}{|l|l|}
\hline ¿Cómo está? & ¿Cómo es? \\
\hline $\begin{array}{l}\text { Hoy es lunes y Elena está un } \\
\text { poco cansada, pero también está }\end{array}$ & Elena es tranquila. \\
\hline & \\
\hline & \\
\hline $\begin{array}{l}\text { Ahora discutid en grupo: ¿Cuándo se usa „estar+Adjetivo“ y cuándo „ser+ Adjetivo“? } \\
\text { Formad juntos una regla. }\end{array}$ \\
\hline
\end{tabular}

Die Aufgabe kann als task mit geringem Komplexitätsgrad bezeichnet werden. Sie erfordert ein planvolles Vorgehen, ist an einer realitätsnahen Problembewältigung (eine Person vorstellen) orientiert und durch die in ihr angelegten Aushandlungsprozesse in der Kleingruppe sprachlich in Grenzen realisierungs- und bedeutungsoffen. 
Aufgabenbearbeitung (s. o., Kap. 2.1, Transkriptauszug, zur besseren Verständlichkeit hier noch einmal als Fußnote ${ }^{19}$ )

Auf der kommunikativen Sinnebene gelingt Lisa die Umsetzung der Personenbeschreibung teilweise. Die beiden Sätze Esteban es no bueno en mates (Z. 103) und Also Estebans actividades después de las clases @ es fútbol y volleyball (Z. 111) erfüllen den kommunikativen Auftrag trotz einiger Grammatikprobleme (Wortstellung bei Verneinung, Genitiv mit de, Pluralangleichung des Verbs). Die Kommunikationspartner erfahren, dass Esteban nicht gut in Mathe ist und dass er nach der Schule Fußball und Volleyball spielt. Das Selbstmonitoring funktioniert nicht, obwohl eine Ahnung vorhanden ist, dass im ersten der beiden Sätze etwas nicht ,richtig formuliert“ ist.

Auf der Sinnebene der Lernersprache lässt sich die dreiphasige Diskursbewegung aus Orientierung (Planung), Ausführung und Evaluation (Kontrolle) rekonstruieren. Lisa führt Ahmeds Anweisung aus, wobei das code switching ins Auge fällt: Schon beim ersten Satz wird die deutsche Satzstruktur verwendet, der Satz ist sozusagen eins zu eins aus dem Deutschen übertragen worden, d.h. Lisa

19 Klasse 8, Gruppe Blau, Passage „Esteban“, Z. 69 bis 119:

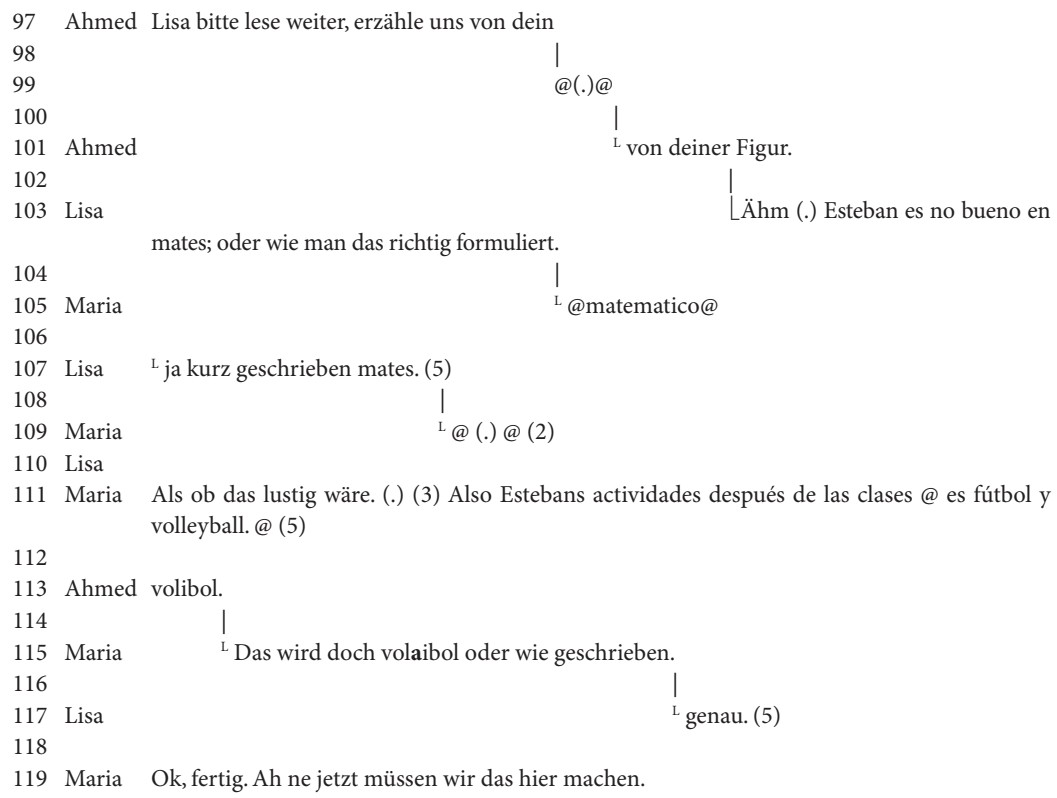


spricht Spanisch und denkt dabei möglicherweise auf Deutsch. Dann evaluiert sie ihre Sprechhandlung übergangslos auf Deutsch („oder wie man das richtig formuliert"). Auch der zweite Satz hat eine deutsche Struktur. Die Kontrolle ist bereits eingebettet in eine Diskursbewegung, die Ahmed und Lisa einbezieht und die Aussprache und Schreibweise des Wortes volleyball betrifft. Mit der rituellen Konklusion („Ok, fertig.“) erfolgt ein Wechsel auf der sozialen Ebene der Kommunikation, denn Maria übernimmt nun ihrerseits die Rolle der Moderatorin.

Marias Lernersprache bewegt sich global auf dem Niveau A2 des GeR. Indizien dafür sind auch, dass sie eine selbst geschriebene Vorlage abliest und beim Formulieren das Deutsche stark als Mittler für ihre Sprachproduktion benutzt. Ihre aktuelle Entwicklungszone könnte durch komplexere Aufgabenstellungen, durch scaffolding-Materialien und Sprachlernstrategien wie Wörterbuchbenutzung und Interkomprehension (Meißner \& Tesch 2010) erweitert bzw. eine individuelle nächst liegende Entwicklungszone erreicht werden.

Auf die verschiedenen Rahmungen durch die grundsätzlich gegebene Orientierung an der Erfüllung der Aufgabe, die Peer-Rivalität und auf die thematische Rahmung wurde bereits in Kapitel 2.1 hingewiesen. In dieser konkreten Gruppe (Ahmed, Lisa und Maria) ist eine starke Identifikation mit dem Merkmal „NichtGutsein-in-Mathe" zu beobachten bzw. indirekt auch eine Identifikation mit der Person Estebans, einer Figur aus dem Lehrbuch. Diese vier Orientierungsmuster herauszuarbeiten und mit den Orientierungen anderer Gruppen innerhalb dieser Gruppenarbeit zu vergleichen, wäre somit das Ergebnis einer forschenden Beschäftigung mit dieser Aufgabenbearbeitung.

Wie lässt sich nun die Beobachtung und Analyse von Aufgabenbearbeitungen forschungsmethodisch einbetten und welche forschungsmethodologischen Herausforderungen gilt es dabei zu bewältigen? Ich beschließe dieses Kapitel mit einer methodologischen Reflexion zu diesen beiden Fragen. In den bisher vorgestellten Unterrichtsepisoden, sowohl der Episode „Beschreibung des Werbefotos eines Kulturkaufhauses" im Französischunterricht einer zehnten Klasse als auch in der Episode „Beschreibung der Person Estebans“ im Spanischunterricht einer achten Klasse findet eine Identifikation mit dem thematischen Lerngegenstand statt. Diese Identifikation könnte somit das tertium comparationis einer forschenden Heransgehensweise im Sinne der Dokumentarischen Methode bilden. Dies jedoch wäre ein eher schwaches gemeinsames Drittes. Da Identifikation mit dem Lerngegenstand kann generell auf einem Kontinuum von ,gar nicht ' bis ,stark verortet werden; es ist jedoch in einer rekonstruktiven Untersuchung weniger die Frage, ob oder wie stark diese Identifikation stattfindet, sondern auf welche Art und Weise, in welcher typischen bzw. habituellen Ausprägung, in welchen 
gruppenhaften Sinnzuweisungen. Dafür wäre es erforderlich, homologe, gleichgelagerte Fälle miteinander zu vergleichen, also etwa die Bearbeitung der Werbefoto-Aufgabe in verschiedenen Französisch-Klassen unterschiedlicher Schulen und Jahrgangsstufen. Im Ergebnis könnte dann eine dreifache verschachtelte Typik im Französischunterricht einer gymnasialen Mittelstufenklasse herauskommen, wie sie ansatzweise bei Tesch (2010) herausgearbeitet wurde: z. B. eine Typik der habituellen Lernarrangements bzw. sozialen Lehr-/Lernformen sowie eine Typik habitueller lernersprachlicher Erwerbsprozesse. Weitere und ausgearbeitete Beispiele zur Analyse der Verständigung über einen Text finden sich in Kap. 4.1.

Die Erforschung des Fremdsprachenunterrichts mit der Dokumentarischen Methode darf jedoch nicht auf der Ebene der Einzeltypen innerhalb einer bestimmten Unterrichtsstunde stehen bleiben. Um den Habitus einer Klasse auf einer höheren Ebene zu erfassen, nämlich auf der Ebene des Klassen- oder Gruppenverhaltens als Ganzheit, ist es erforderlich, die Klasse über mehrere Stunden hinweg zu begleiten, dabei die wiederkehrenden Verständigungsmuster herauszuarbeiten und diese wiederum mit den immer wiederkehrenden Verständigungsmustern anderer Klassen zu vergleichen (s. Tesch 2010, Bracker 2015). Dabei kann der milieuhafte Gesamthabitus einer Klasse sichtbar werden, wie er gegebenenfalls systemisch bereits in einem bestimmten Schul- und Schulorganisationstyp, in einem bestimmten Bildungsgang und landesweit in einer bestimmten Bildungsgangkultur angelegt ist.

Ein theoretisches Problem stellt in diesem Zusammenhang die Individualität der Lernersprache im Gegensatz zur Kollektivität bzw. Sozialität des impliziten Wissens dar (vgl. Kap. 2.1). Die Lernersprache entwickelt sich einerseits durchaus durch soziale Mediation, d.h. unter anderem durch kooperative sprachliche Konstruktionsprozesse mit Peers und Lehrperson. Andererseits ist die Lernersprache auch Produkt der individuellen Sprachenbiografie, abhängig von Einflüssen und Kulturen, die außerhalb des konjunktiven Erfahrungsraumes der Klasse liegen, wie z.B. Mehrsprachigkeit im Elternhaus, sprachlich-musikalische Förderung durch Hobbys (z.B. Musikunterricht), Urlaubsreiseverhalten der Familie u.v .a. m. Diese wirken biografisch über viele Jahre zusammen und formen sozusagen die lernersprachliche Persönlichkeit mit. Die Individualität speist sich ihrerseits in begrenztem Umfang in die Gesamtentwicklung aller Lernersprachen einer Klasse ein. Man könnte das Verhältnis der lernersprachlichen Individualität zur lernersprachlichen Kollektivität mit dem Verhältnis des sichtbaren Teils eines Eisbergs zu seinem unsichtbaren Teil vergleichen. Der größte Teil der individuellen Lernersprache bleibt im Unterrichtsgeschehen unsichtbar. Er 
kann jedoch ansatzweise durch die Diskursanalyse mit der Dokumentarischen Methode herausgearbeitet werden. Mit Hilfe des narrativen Interviews können gegebenenfalls Hinweise auf die individuelle Sprachenbiografie (vgl. Kapitel 2.4) und somit weitere Elemente für das Verstehen gruppenhafter Verständigung im Fremdsprachenunterricht ermittelt werden.

Im unterrichtlichen Konstruktionsprozess können die unterschiedlichen lernersprachlichen Performanzen auf der intentionalen Sinnebene der Aufgabenstellung, auf der kommunikativen Sinnebene (das Was der sprachlichen Handlung) und auf der Dokumentarischen Sinnebene (das Wie dieser Handlungen) beschrieben und analysiert werden. Bleibt die Lernersprache in der rein praktischen Anwendung verhaftet, so entspricht sie strukturanalytisch betrachtet der kommunikativen, atheoretischen Sinnebene (common sense). Wird sie freilich im Sinne von Sprachbewusstheit reflektiert bzw. kommentiert, transzendieren die Sprecher den kommunikativen Sinn und gelangen zu Vorstufen einer Interpretation ihrer eigenen Äußerungen. Das bedeutet, dass sie bereits eine theoretische Ebene der Verständigung erreicht haben, die jedoch lediglich den lernersprachlichen, nicht den sozialen Sinn ihres sprachlichen Handelns betrifft.

Zusammengefasst ging es im Kapitel 2.3 um die Möglichkeit, mit der Dokumentarischen Methode die sozialen und lernersprachlichen Interaktionen der Aufgabenbearbeitung im Fremdsprachenunterricht $\mathrm{zu}$ erforschen, das heißt, Transformationen zu untersuchen, die der intendierte Sinne einer Aufgabe im Zuge der Bearbeitung im Unterricht erfährt. Texte jeglicher Art und Modalität können im Fremdsprachenunterricht als Impulse für planvolles Lernhandeln genutzt werden. Experten wie z.B. Redaktionsteams oder Lehrpersonen führen diesbezüglich eine erste Transformation durch, indem sie das Textpotential zu einem Aufgabenpotential formen. Auch Lernende selbst können sich Aufgaben stellen. Dann, bei der Bearbeitung, erfolgt eine zweite, viel wesentlichere Transformation, die dieses in die Aufgabe eingelagerte didaktische Wissen in Lernendenwissen verwandelt. Die Ergebnisse könnten vielfältiger nicht sein, da nun Lehrhandlungen, Lernhandlungen und textuell-mediale Wirkungen interagieren. Wie diese Interaktionen rückschauend von den Akteuren wahrgenommen werden und wie diese Wahrnehmung analysiert werden kann, wird im folgenden Kapitel beschrieben.

\subsection{Verständigung über erlebten Fremdsprachenunterricht, über das Lehren und über das Lernen von Sprachen}

In den beiden vorangegangenen Teilkapiteln wurden methodologische Grundlagen für die Analyse der Verständigung durch und über Texte bzw. über Aufgaben vorgestellt. In diesem Teilkapitel geht es darum, dass auch auf einer 
von konkreten Texten losgelösten Ebene Verständigung über das Fremdsprachenlehren und -lernen stattfindet, wenn nämlich der Unterricht als solcher oder die biografische Sicht auf das Lehren und das Lernen von Sprachen Gegenstand der Verständigung sind. Die Verständigung über erlebten Unterricht findet beispielsweise in Evaluationsphasen nach einer schulischen Unterrichtseinheit statt. Die Verständigung über das Lehren von Sprachen kann in Hochschulseminaren stattfinden, aber auch beispielsweise in Fachkonferenzen an Schulen. Für Forschungszwecke eignet sich in diesem Zusammenhang neben der Gruppengesprächsanalyse auch das narrative Interview, insbesondere in Form von Interviews mit Lehrerinnen und Lehrern sowie von sprachenbiografischen Interviews.

Die methodologischen Grundlagen der Gruppengesprächsanalyse wurden im vorangehenden Kapitel 2.1 am Beispiel einer unterrichtlichen Gruppenarbeit („Esteban“) vorgestellt. Die folgenden Ausführungen sind daher auf das narrative Interview fokussiert. Im Unterschied zur Verständigung im Unterricht entfällt bei der Verständigung über Unterricht und über das Lehren und Lernen von Sprachen die Analyse der Lernersprache, was zu einer methodischen Vereinfachung führt. Wir bewegen uns nun ganz im Rahmen der rekonstruktiven Sozialforschung und können den Sonderfall der partiell oder vollständig zielsprachigen Interaktion daher an dieser Stelle ausklammern.

Interviews mit Lehrenden und Lernenden wurden in der Vergangenheit z.B. mit dem Forschungsansatz der Subjektiven Theorien untersucht und ausgewertet. Kallenbach (1996) untersuchte Subjektive Theorien anhand von Interviews, die sie bei Oberstufenschülern durchführte und eröffnete interessante Einblicke in das Denken von Lernenden im Hinblick auf bestimmte Sprachlernzugänge und damit auch im Hinblick auf Einstellungen, die Lern(er)verhalten im Unterrichtsgeschehen erklären könnten. Caspari (2003) untersuchte auf Grundlage von Interviews die Sprachlernerfahrungen von Lehrkräften, ihr persönliches Verhältnis zu Sprache(n), Land und Leuten, allgemeine Auffassungen zum Sprachenlernen und zum Fremdsprachenunterricht, Aufgaben- und Rollenbestimmungen (Vermittler, Erzieher, Lernberater), Probleme als Fremdsprachenlehrende sowie äußere Umstände und Bedingungen des Lehrerwerdens und Lehrerseins. Trautwein (2014) untersuchte mit Hilfe einer Interviewstudie fremdsprachliche Identitätskonstruktionen von Oberstufenschülerinnen und -schülern. Strohns Studie (2015) zur Binnendifferenzierung im Englischunterricht widmet sich der Lehrerperspektive bezüglich des Themenbereichs Binnendifferenzierung und Individualisierung und nutzt dabei u. a. auch die Methodik der Subjektiven Theorien. 
Neben dem Forschungsansatz der Subjektiven Theorien erscheint das narrative Interview als ein methodologisch besonders geeignetes Verfahren zur Erforschung von Fremdsprachenlehr- und Sprachenlernphänomenen, da es einen empirischen Zugang zu lebensgeschichtlichen Erfahrungen eröffnet. Diese Erfahrungen können im Kleinformat bereits bei der Evaluation von erlebtem Unterricht und Sprachlernerfahrungen im Rahmen einer Gruppendiskussion einfließen, wenn sich Sprecher in narrativen Passagen zu einem bestimmten Aspekt äußern. Sie können aber auch in das Großformat der biografischen Erzählung münden, etwa wenn das narrative Interview dazu eingesetzt wird, Sprachenbiografien zu erheben, zu analysieren und mit dem Ziel einer Typenbildung $\mathrm{zu}$ vergleichen. Besondere Betonung liegt wiederum auf der „spontanen, vom Erzähler nicht vorher zurecht gelegten Stegreiferzählung“" (Kleemann et al. 2009: 66), da sie den Erzähler in bestimmte Zugzwänge des Erzählens verwickelt. Die Stegreiferzählung unterliegt nach Schütze $(1976,1983)$ der Homologieannahme, d.h. der angenommen Übereinstimmung von formaler sequentieller Struktur und subjektiven „Erfahrungsaufschüttungen“ (ibd.). Oder einfacher formuliert: In der Struktur der Erzählung spiegelt sich das Erleben der Person. Die Analyse zielt somit darauf ab, über die Rekonstruktion der Sequenzialität zugleich auch die Formung des Lebenslaufs herauszuarbeiten. Schütze (1983) ermittelte in seinen Arbeiten vier idealtypische Prozessstrukturen von Lebensläufen, die im Folgenden exemplarisch ausgeführt und auf den Untersuchungsgegenstand Lehrendenbiografien übertragen werden:

a) Institutionelles Ablaufmuster,

b) Biografisches Handlungsmuster,

c) Verlaufskurve,

d) Biografischer Wandlungsprozess.

Beim „institutionellen Ablaufmuster" steht das Individuum fest eingebunden in institutionellen Normen, denen es sich unterwirft. Dies wäre beispielsweise bei Fremdsprachenlehrenden der Fall, die die klassischen Laufbahnstationen Lehramtsstudium, Vorbereitungsdienst und Eintritt in den Schuldienst, Verbeamtung und gegebenenfalls interner Aufstieg zum Fachkonferenzvorsitzenden und zu einer Funktionsstelle durchlaufen.

Im „biographischen Handlungsmuster" hingegen sind bestimmte Abweichungen von der Norm gegeben. Das Individuum beendet beispielsweise eine bereits begonnene Laufbahn in einem anderen Beruf, erkennt die Berufung für das Lehramt, studiert mit Ende dreißig die Wunschfächer Lehramt Geschichte und Lehramt Anglistik und wird schließlich mit Mitte vierzig Lehrer im Angestelltenverhältnis. 
Die „Verlaufskurve“ beschreibt „das Prinzip des Getriebenwerdens durch sozialstrukturelle und äußerlich-schicksalhafte Bedingungen der Existenz" (Schütze 1983: 288). Damit sind Lebensumstände gemeint, die das Individuum nicht selbst zu beeinflussen vermag und die in eine Art Ausweglosigkeit oder auch überraschende Wende münden können. Eine Englischlehrerin beispielsweise sieht sich mit Anfang fünfzig beruflich in einer Sackgasse; eine interne Weiterentwicklung an ihrer Schule scheint unmöglich, gleichzeitig erstickt sie in den Routinen des Berufsalltags. Auch privat sieht sie keinen Ausgleich. Die Wende erfolgt, als sie auf eine unerwartete Stellenausschreibung als Fachberaterin stößt und sich erfolgreich bewirbt.

Der „biographische Wandlungsprozess“ schließlich ist dadurch gekennzeichnet, dass das Individuum aus einer reaktiven erleidenden Haltung herausfindet und neue selbstbestimmte Handlungsmöglichkeiten erfährt. Dies könnte etwa im Anschluss an die Verlaufskurve bei jener Englischlehrerin eintreten, die in ihrer neuen Tätigkeit als Fachberaterin ihr Interesse am wissenschaftlichen Schreiben entdeckt, über einige Jahre hinweg kleinere Fachartikel verfasst und am Ende mit einer berufsbegleitenden Promotion am wissenschaftlichen Diskurs teilnimmt.

Diese vier idealtypischen Prozessstrukturen können Forschenden als Orientierung dienen. Sie helfen, das Datenmaterial des narrativen Interviews inhaltlich vor eine Folie zu halten, die es erlaubt, Übereinstimmungen und Abweichungen von der Normerwartung zu identifizieren und damit die „biografische Gesamtformung" (Bohnsack 2014: 97) eines Falles herauszuarbeiten.

Kallmeyer \& Schütze (1976) legten im Rahmen ihrer erzähltheoretischen Fundierung auch ein Regelsystem des formalen Aufbaus von Stegreiferzählungen vor, das folgende drei Aspekte umfasst. Ich gebe hier in einem längeren Zitat die Zusammenfassung in Bohnsack (2014: 95 f.) wieder, da die dort erwähnten Strukturelemente eine grundsätzliche Relevanz für die Analyse narrativer Interviews wie auch längerer narrativer Passagen in Gruppengesprächen aufweisen (s. Kap. 4.2):

- Gestaltschließungszwang: Auch ohne eigene Vorabkenntnis des in einer Erzählung inhaltlich Dargestellten verfügen wir (aufgrund des intuitiven Wissens über den formalen Aufbau von Erzählungen) über die Kompetenz, darüber entscheiden zu können, ob eine Erzählung oder Teilerzählung abgeschlossen - d.h. ihre, Gestalt geschlossen' ist oder nicht.

- Relevanzfestlegungs- und Kondensierungszwang: Da die Erzählzeit begrenzt ist [...] muss er [der Erzähler] sich - was die Gesamterzählzeit wie auch die der Teilerzählungen anbelangt - auf das Wesentliche beschränken - dennoch 
aber die Gestalten schließen. Er muss also die Erzählung und ihre Teilerzählungen entsprechend verdichten, kondensieren.

- Detaillierungszwang: Ist der Erzähler erst einmal auf bestimmte biografisch relevante Ereignisse zu sprechen gekommen, so ist er zum Zwecke der Plausibilisierung des Geschehenen und um das Geschehene in den Fortlauf der Erzählung plausibel einfügen zu können, bisweilen dazu ,gezwungen', detaillierter auf den Kontext der angesprochenen Ereignisse einzugehen. Es werden dann aber möglicherweise gerade dadurch Handlungs-, Entscheidungs- und Verlaufsmuster sichtbar, die der Erzähler ansonsten übergangen hätte - sei es, dass er sie vergessen hat oder dass er sie verbergen wollte.

Diese Zwänge gilt es bei der Analyse narrativer Interviews zu beachten, da die Verständigung anders als bei der Gruppengesprächsanalyse nicht mit einem oder mehreren Kommunikationspartnern erfolgt, sondern selbstläufig innerhalb einer einzigen Narration. D.h. die Strukturierung des Diskurses erfolgt nicht über die Reaktionen von Gesprächsteilnehmern, sondern über narrationsinterne Zwänge. Anders als bei der - innerhalb der Gruppengesprächsanalyse von Bohnsack entwickelten - Abfolge der Analyseschritte wird bei der Analyse des narrativen Interviews auf Grundlage der oben genannten vier idealtypischen Prozessstrukturen zunächst der Fall in seiner Gesamtgestalt herausgearbeitet und dabei durch fallinternen Vergleich die „biografische Gesamtformung“ entwickelt. Dies gründet in der Tatsache, dass beim biografischen Interview bereits eine in sich geschlossene Gestalt - ein Persönlichkeitstypus - vorliegt. Erst im Anschluss daran setzt der Vergleich mit anderen Fällen ein, der es erlaubt, weitere Typen - etwa auf der Ebene einer Geschlechts- oder Generationentypik zu bestimmen.

Wiederum im Gegensatz zur Gruppendiskussion liegt dem narrativen Interview kein gemeinsames Erleben Anwesender zu Grunde: Der Interviewer begegnet einem Erzähler als Einzelindividuum, das seine Geschichte als Einzelgeschichte erzählt. Diese kann zwar mit den Geschichten anderer Erzähler verglichen werden, doch ist der Erfahrungsraum des Erzählers zunächst individuell und somit primär persönlichkeitstypisch. Die ,Anderen' treten im narrativen Interview als implizite Referenz auf und müssen durch die Analyseschritte als normative Bezugsgröße erst herausgearbeitet werden. Sie können dadurch auch zu einer Bezugsgröße als Gruppe werden, die es ermöglicht, die Individualität des Falles überhaupt zu erkennen. Natürlich ist es möglich, auch im Bildungskontext durch narrative Interviews $u$. a. Generationstypisches herauszuarbeiten, wenn nämlich verschiedene Fälle auf einschneidende und prägende Veränderungen hin analysiert werden: aus Schülersicht etwa die Einführung des achtjährigen 
Gymnasiums (G8) oder aus Sicht der Unterrichtenden z.B. die Einführung des Inklusionsunterrichts. Es können in narrativen Interviews darauf bezogene konjunktive Erfahrungen verglichen werden, ohne dass ein gemeinsames Erleben unmittelbar gegeben ist. Mithin bleibt festzuhalten, dass beim narrativen Interview vom Einzelfall abstrahiert werden muss, um das Konjunktive bzw. Kollektive zu erfassen, während der Forschende bei der Gruppengesprächsanalyse dem gemeinsamen Rekonstruieren kollektiver Erfahrungen direkt beiwohnt.

Eine Verbindung der Dokumentarischen Methode und des narrativen Interviews wird auch von Nohl $(2005,2012)$ vorgenommen. Er arbeitet eine Parallele zwischen dem konjunktiven Wissen in der biografischen Stegreiferzählung und dem konjunktiven Erfahrungsraum bei der Gruppengesprächsanalyse einerseits und von Erzählsequenzen in der biografischen Stegreiferzählung und Handlungssequenzen bei der Gruppengesprächsanalyse andererseits heraus. Dies bedeutet nach seiner Auffassung, dass auch die dokumentarische Sequenzanalyse auf die Analyse narrativer Interviews übertragen werden kann und dass Abschnitte einer Erzählung, sofern sie innerhalb eines gemeinsamen, homologen Orientierungsrahmens liegen, aufeinander bezogen werden können, um daraus eine Regelhaftigkeit abzuleiten. Ein narratives Interview kann dann als ein Fall betrachtet werden, der mit anderen Fällen oder Interviews verglichen werden kann. Liegt ein gemeinsames zu vergleichendes Drittes vor - bei Lehrerbiografien beispielsweise eine erfolgte oder ausbleibende Anerkennungspraxis - so ist es möglich, daraus eine übergreifende Typik (z.B. Geschlechter- oder Generationstypik) zu entwickeln. So könnten beispielsweise die Phasen eines Wandlungsprozesses miteinander verglichen werden: Ein Lehrer und seine Kollegin erfahren beide ein Ausbleiben beruflicher Anerkennung. Während sich Lehrer X daraufhin von seiner Schule abwendet und eine Versetzung in den Auslandsschuldienst beantragt, betreibt Kollegin $\mathrm{Y}$ den Versuch, sich hierarchisch zu verändern und bewirbt sich um eine Funktionsstelle an ihrer Schule.

Voraussetzung für eine valide Typenbildung ist jedoch die hinreichende Variation des samplings. Um beim obigen Beispiel zu bleiben: Aus einem Fall ,Lehrer $\mathrm{X}^{`}$ und einem Fall ,Kollegin $\mathrm{Y}^{`}$ lässt sich allein weder eine Geschlechtstypik noch eine Generationentypik ableiten, allenfalls Hinweise auf eine denkbare Typik, die durch weitere Fälle erhärtet werden müssten. Die Typik kann nun wie bei der Gruppengesprächsanalyse zu einer Typologie erweitert werden, wenn es gelingt, die Überlagerung einer Typik durch andere Typiken und das Verschachteltsein mehrerer Typiken innerhalb ein und derselben Biografie nachzuweisen.

In Kapitel 2.4. wurde das narrative Interview als methodischer Rahmen für die Erforschung der Verständigung über das Lehren und Lernen von Sprachen 
vorgestellt. Das von Schütze entwickelte Analyseverfahren beruht auf der Homologieannahme: Die Strukturen der retrospektiven Erzählung spiegeln die Strukturen der ursprünglichen Erfahrung selbst, sind also homolog zu ihnen. Es gilt somit, den Erzählungskern herauszuarbeiten, d.h. die Passagen, in denen sich idealtypische Prozessstrukturen von Lebensläufen zeigen, z. B. institutionelle Ablaufmuster in Lehrerbiografien. Dies wird möglich, indem die Erzählung und damit der Lebenslauf eines Individums mit der Erzählung eines anderen Individuums verglichen wird, aber auch, indem bereits innerhalb einer konkreten Erzählung das Verhalten des Individuums gegenüber Normerwartungen rekonstruiert wird.

Bevor wir im drittel Kapitel den Blick auf methodische Aspekte richten, auf die konkreten Arbeitsschritte der Dokumentarischen Methode, fasse ich das auf die Methodologie fokussierte zweite Kapitel noch einmal kurz zusammen. Es wurden zentrale methodologische Bezüge der Anwendung der Dokumentarischen Methode in der Fremdsprachenforschung vorgestellt, nämlich zum einen die begrifflich-konzeptuale Verortung in der Wissenssoziologie (methodisch kontrolliertes Fremdverstehen, Rahmenorientierungen, Typenbildung, Milieu und konjunktiver Erfahrungsraum) bzw. der Linguistik (Diskursanalyse, Lernersprache) sowie Umrisse eines sozial-interaktionalen Modells des Fremdsprachenlehrens und -lernens. Zum anderen ging es in diesem zweiten Kapitel um die Frage, welche Ebenen der Verständigung der Fremdsprachenunterricht bzw. das Fremdsprachenlernen aufweist und wie sie mit Hilfe der Dokumentarischen Methode zugänglich gemacht werden können.

Das nun folgende dritte Kapitel ist den konkreten Arbeitsschritten der Dokumentarischen Methode gewidmet. 
Bernd Tesch - 978-3-631-69542-5

Downloaded from PubFactory at 01/11/2019 10:14:38AM

via free access 


\section{Arbeitsschritte der Dokumentarischen Methode}

\subsection{Gruppendiskussion und Unterrichtsgespräch}

Ziel dieses Kapitels ist es, Forschende in die Arbeitsschritte der Dokumentarischen Methode, bezogen auf die Fremdsprachenforschung, einzuführen. Sie wurden im Hinblick auf die Gruppengesprächsanalyse allgemein im Verlauf des Forschungsprojekts „Kollektive Orientierungen in Gruppen Jugendlicher" von 1984 bis 1987 (Mangold \& Bohnsack 1988) entwickelt: erstens die teilnehmende Beobachtung und die Audioaufzeichnung von Gruppengesprächen inklusive Transkription, zweitens die formulierende Interpretation, drittens die reflektierende Interpretation, viertens die Fallbeschreibung und fünftens die Typenbildung. Häufig bleibt der erste Arbeitsschritt - die teilnehmende Beobachtung und die Audioaufzeichnung inklusive Transkription - unerwähnt. Auf Grund seiner Bedeutung für die Erforschung des Unterrichts wird diesem Arbeitsschritt nun zusammen mit der Art und Weise der Durchführung von Audioaufnahmen und Transkriptionen im Folgenden ein eigener Abschnitt gewidmet. Die fünf Arbeitsschritte sind ansonsten, bezogen auf fremdsprachliche Untersuchungsgegenstände, grundsätzlich identisch mit den Arbeitsschritten bezogen auf nicht fremdsprachliche Untersuchungsgegenstände. Die Analyse der Lernersprache, die eine wichtige Erweiterung bei der Untersuchung fremdsprachenunterrichtlicher Interaktionen betrifft, ist in die Analyseschritte integriert.

Hinsichtlich der Erforschung des narrativen Interviews sowie multimodaler Texte (Beispiel Comic) sind einige Modifikationen erforderlich, auf die in den Kapitel 3.2 und 4 jeweils hingewiesen wird.

\subsubsection{Teilnehmende Beobachtung, Audioaufzeichnung und Transkription}

\section{Teilnehmende Beobachtung}

Die teilnehmende Beobachtung des Unterrichts und die akustische Aufzeichnung des Unterrichtsgeschehens umfassen die Phase der Feldarbeit, mit der Transkription des aufgezeichneten Unterrichts beginnt die Auswertung. Bohnsack weist auf die drei Funktionen der teilnehmenden Beobachtung im Rahmen der 
Dokumentarischen Methode hin (2014: 133 ff. $)^{20}$. Übertragen auf die Beobachtung des Fremdsprachenunterrichts lassen sie sich folgendermaßen spezifizieren. Erstens liefert die teilnehmende Beobachtung wichtige Kontextinformationen, die den reinen Audiodaten nicht zu entnehmen sind, wie z.B. die Platzierung der Unterrichtsstunde im Tagesablauf einer Klasse und die Auswirkungen auf die Interaktionen. So kann es sein, dass eine Klasse gerade vom Sportunterricht kommt und erschöpft wirkt oder in der Folgestunde eine Mathematikarbeit schreibt, die bereits auf die Stimmung drückt. Auch die Beschaffenheit des Klassenzimmers sollte notiert werden (Belüftung, Beleuchtung, Raumgröße, mediale Ausstattung und Platzierung der Medien) sowie die Sitzordnung in der Klasse. Zweitens liefert die teilnehmende Beobachtung Informationen über „nonverbale Handlungsdimensionen“ (ibd.: 134): Gestik, Mimik, Proxemik, Emblemik (Frisur, Kleidung, Tatoos, Piercings etc.) der Handelnden. Drittens erlaubt die teilnehmende Beobachtung, Lehr-Lernrituale einer Klasse wahrzunehmen, die den rein verbalen Aufzeichnungen möglicherweise verborgen bleiben: z.B. die Ritualität einer Begrüßung und eines Arbeitsbeginns (scheinbar übergangslos oder durch Signalhandlungen initiiert), die Ritualität einer Übung (z. B. Reihenübungen, alphabetisches ,Drankommen'), die Ritualität eines Stundenabschlusses, die physische Präsenz der Lehrperson (z.B. Standortwahl und -veränderung, stimmliche Präsenz bzw. Stimmvolumen), die zeitliche Kontrolle einer Unterrichtsstunde durch die Lehrperson.

Somit ist klar, dass die teilnehmende Beobachtung nicht nur eine wichtige Rolle bei der Unterrichtsforschung spielt, mehr noch als bei vielen nicht unterrichtlichen Gruppendiskussionen, sondern auch eine komplementäre Funktion im Sinne der methodischen Triangulation (Flick 2003) ausübt. Sie unterstützt Forschende dabei, bestimmte Unterrichtsstunden schwerpunktmäßig für die

20 Auf die Berücksichtigung von Videoaufzeichnungen an Stelle von oder neben Audioaufzeichnungen wird an dieser Stelle verzichtet. Für die Erforschung des Unterrichtsalltags stellt die Filmspur als Datenquelle (embodied language, gestische und mimische Interaktion) zwar eine sehr wünschenswerte Ergänzung zur reinen Tonspur dar, ist aber genehmigungsrechtlich außerordentlich hohen Hürden ausgesetzt und impliziert gleichzeitig einen invasiveren Eingriff als die Tonaufzeichnung. Schülerinnen und Schüler aber auch Lehrerinnen und Lehrer sind bei der Anwesenheit einer Videokamera erfahrungsgemäß geneigt, ihr Verhalten deutlicher zu verändern, als sie es bei Audioaufnahmen tun. Dies wiederum wirft das Problem einer methodischen Kontrolle, z.B. durch kommunikative Validierung auf. Generell stellt das Problem des Umgangs mit der durch Unterrichtsbeobachtung erzeugten Verunsicherung der Beobachteten nach meinem Überblick theoretisch noch ein Desiderat dar. 
Analyse heranzuziehen bzw. anderen vorzuziehen und innerhalb einer Unterrichtsstunde eine sinnvolle Auswahl von Sequenzen vorzunehmen, die einer reflektierenden Interpretation unterzogen werden sollen (vgl. Tesch 2010).

Allerdings, so stellt Bohnsack fest, ,gelingt die Integration unterschiedlicher Methoden im Sinne einer Triangulation nur dann, wenn sie durch ihnen gemeinsame methodologische und metatheoretische Grundlagen verbunden sind“ (Bohnsack 2014: 134). Die teilnehmende Beobachtung im Rahmen der rekonstruktiven Fremdsprachenforschung mit der Dokumentarischen Methode ist somit den gleichen wissenssoziologischen Grundannahmen zu unterwerfen wie die Textanalyse. Dies betrifft insbesondere die Annahme, dass sich das Wissen der Forschenden grundsätzlich nicht vom Wissen der Beforschten unterscheidet und dass folglich die Produktionsgesetzmäßigkeiten der Verständigung im Unterricht denen der wissenschaftlichen Erforschung von Unterricht entspricht (anders Bruner 1985, der in den beiden Wissensmodi auch zwei völlig verschiedene Weisen des Denkens und der Wahrnehmung sieht). Die Beobachtungsprotokolle sind folglich demselben Analyseverfahren und denselben Analyseschritten zu unterziehen wie die Analyse der Audiotexte, so dass auf dieser Grundlage auch hier eine Rekonstruktion erfolgen kann.

\section{Audioaufzeichnung}

Die Audioaufzeichnung von Unterrichtsstunden erfolgt i. d. R. mit Diktiergeräten, die auf Schülertischen ausgelegt werden. Nach Möglichkeit sollten so viele Geräte benutzt werden, wie es die Forschungsfrage nahelegt. Sollen beispielsweise Gruppenarbeitsphasen untersucht werden, so ist es wünschenswert, bei allen beteiligten Gruppen ein oder zwei Diktiergeräte zu platzieren. Die Verwendung eines zweiten Diktiergeräts hat den Vorteil, dass eventuelle Dekodierunsicherheiten bei der einen Aufnahme durch das Gegenhören der zweiten Aufnahme beseitigt werden können.

Die Durchführung von Audioaufnahmen mit Hilfe von Diktiergeräten stellt einen Eingriff in die natürliche Lernumgebung dar, so wie auch bereits die Anwesenheit des Beobachters. In der Forschungspraxis kann dabei folgendes Phänomen auftreten: Die Beobachteten lassen sich phasenweise vom Diktiergerät ablenken und widmen einen (meist geringen) Teil ihrer Verständigung dieser Ablenkung. Sie kommentieren dann u. U. die Anwesenheit des Geräts bzw. die Tatsache, dass ihre Gespräche aufgenommen werden, häufig machen sie diesbezügliche Scherze. Es kann aus den Daten jedoch meist nicht entnommen werden, dass die Tatsache der Audioaufzeichung des Unterrichts einen verzerrenden Einfluss auf die sozialen Beziehungen innerhalb der Klasse bzw. auf die sozialen Aushandlungsprozesse hat. Daher ist zu vermuten, dass die Verständigung 
im Unterricht unter Anwesenheit eines Diktiergeräts ebenso selbstläufig erfolgt wie ohne Diktiergerät. Der Handlungsdruck des Unterrichts lässt die Lernenden (ebenso wie die Lehrperson) in der Regel die Anwesenheit eines ,digitalen Beobachters' vergessen.

Der Forschende erklärt den Schülerinnen und Schülern seine Anwesenheit in der Unterrichtsstunde, erläutert das Verfahren, insbesondere den Aspekt der Transkription und Anonymisierung, und sorgt damit dafür, dass keine unnötige ,Geheimniskrämerei` entsteht. Die Erfahrung zeigt, dass sich Unterrichtende und Unterrichtete umso zwangloser auf die Beobachtungssituation einlassen, je transparenter die Absichten und das Verfahren gemacht werden, was der Validität der Ergebnisse zu Gute kommt.

\section{Transkription}

Mit der Auswahl der zu transkribierenden Unterrichtsstunden und dem Anfertigen eines Transkripts beginnt die Interpretation, denn bereits hier treffen Forschende Entscheidungen darüber, was sie analysieren und wie sie es analysieren. Je genauer und sensibler die Transkription den Diskursbewegungen folgt, um so mehr kann es gelingen, die soziale Dimension verbalen und teilweise nonverbalen Handelns wiederzugeben, wie am unten eingefügten Beispiel sichtbar wird.

Für das Anfertigen von Transkripten bestehen in der qualitativen Sozialforschung verschiedene und teilweise unterschiedliche Regelwerke ${ }^{21}$. Bewährt hat sich bei der Arbeit mit der Dokumentarischen Methode das von Przyborski (2004: 331 ff.) und Bohnsack (2014: 253 f.) vorgeschlagene Transkriptionssystem, das hier näher vorgestellt werden soll.

L Beginn einer Überlappung, d.h. gleichzeitiges Sprechen von zwei

Diskussionsteilnehmern; Markierung eines direkten Anschlusses beim Sprecherwechsel

$」 \quad$ Ende einer Überlappung

(.) kurze Pause, etwa eine Sekunde

(3) Anzahl der Sekunden, die eine Pause dauert

nein betont

nein laut (in Relation zur üblichen Lautstärke des Sprechers/der Sprecherin)

${ }^{\circ}$ nein $^{\circ} \quad$ sehr leise (in Relation zur üblichen Lautstärke des Sprechers/der Sprecher) stark sinkende Intonation

schwach sinkende Intonation

stark steigende Intonation

21 z.B. die Transkriptionskonvention GAT 


\begin{tabular}{|c|c|}
\hline \multirow{2}{*}{ Viellei- } & schwach steigende Intonation \\
\hline & Abbruch eines Wortes \\
\hline Nei:::n & Dehnung; die Häufigkeit von : entspricht der Länge der Dehnung \\
\hline $\mathrm{oh}=$ nee & Wortverschleifung \\
\hline (kein) & Unsicherheit bei der Transkription \\
\hline ( ) & unverständliche Äußerungen \\
\hline (doch) & $\begin{array}{l}\text { unverständliche Äußerungen; die Länge der Klammer entspricht etwa } \\
\text { der Dauer der unverständlichen Äußerung. }\end{array}$ \\
\hline $\begin{array}{l}\text { [Gong] } \\
\text { [stöhnt] }\end{array}$ & $\begin{array}{l}\text { Kommentare bzw. Anmerkungen zu parasprachlichen, nichtverbalen } \\
\text { oder gesprächsexternen Ereignissen; die Länge der Klammer } \\
\text { entspricht im Falle der Kommentierung parasprachlicher Äußerungen } \\
\text { (z.B. Stöhnen) etwa der Dauer der Äußerung. Auch ein Lachen kann } \\
\text { mit Hilfe der doppelten Klammer angezeigt werden. }\end{array}$ \\
\hline$\ldots \ldots$ & Auslassungen im Transkript \\
\hline @Text@ & Text wird lachend gesprochen \\
\hline @(.)@ & kurzes Auflachen \\
\hline @(3)@ & drei Sekunden Lachen \\
\hline$/ / \mathrm{mhm} / /$ & $\begin{array}{l}\text { bei biografischen Interviews: Hörersignal des Interviewers, wenn das } \\
\text { „mhm“ nicht überlappend ist }\end{array}$ \\
\hline & ussionsleiter, Moderator \\
\hline & \\
\hline we & \\
\hline & \\
\hline
\end{tabular}

Wie man sieht, zeigen die verwendeten Satzzeichen in der Transkription keine grammatikalische Funktion an, sondern die Intonation. Diese Kennzeichnung entscheidet häufig über den dokumentarischen Sinn einer Aussage. Nach Punkt oder Fragezeichen wird klein weitergeschrieben. Bezüglich der Großschreibung gilt, dass deutsche Substantive groß geschrieben werden, ebenso das erste Wort beim Einsetzen eines turns.

Im Sinne der Vereinfachung der intersubjektiven Überprüfbarkeit werden die Zeilen nummeriert und die Zählwerkangaben in Minuten und Sekunden notiert. Die transkribierende Person erhält ein Kürzel, das gesamte Transkript einen Codenamen.

Die Namen der Gesprächsteilnehmer werden anonymisiert, z.B. durch Am, $\mathrm{Bm}, \mathrm{Cm}, \ldots \mathrm{Af}, \mathrm{Bf}, \mathrm{Cf}, \ldots$ ( $\mathrm{m}$ für männlich, w für weiblich) oder durch erfundene Namen (Alias). Die erfundenen Namen entstammen dem Kulturkreis der betreffenden Personen. 
Hier ein Auszug aus dem mehrfach zitierten Beispiel aus dem Spanischunterricht einer achten Klasse. Diese sehr detaillierte Transkription wird für die Episoden vorgenommen, die für die reflektierende Interpretation ausgewählt wurden. Es hat sich bewährt, die komplette Unterrichtsstunde zu transkribieren, allerdings genügt im ersten Schritt eine summarische Transkription. Auf ihrer Grundlage sowie auf Grundlage der teilnehmenden Beobachtung sowie des anschließenden mehrfachen Hörens der Audioaufnahmen kann dann die Auswahl der detailliert zu transkribierenden Episoden getroffen werden.

Klasse 8, Gruppe Blau, Passage „Esteban“, Z. 97 bis 119, (5’01” bis 5’42”):

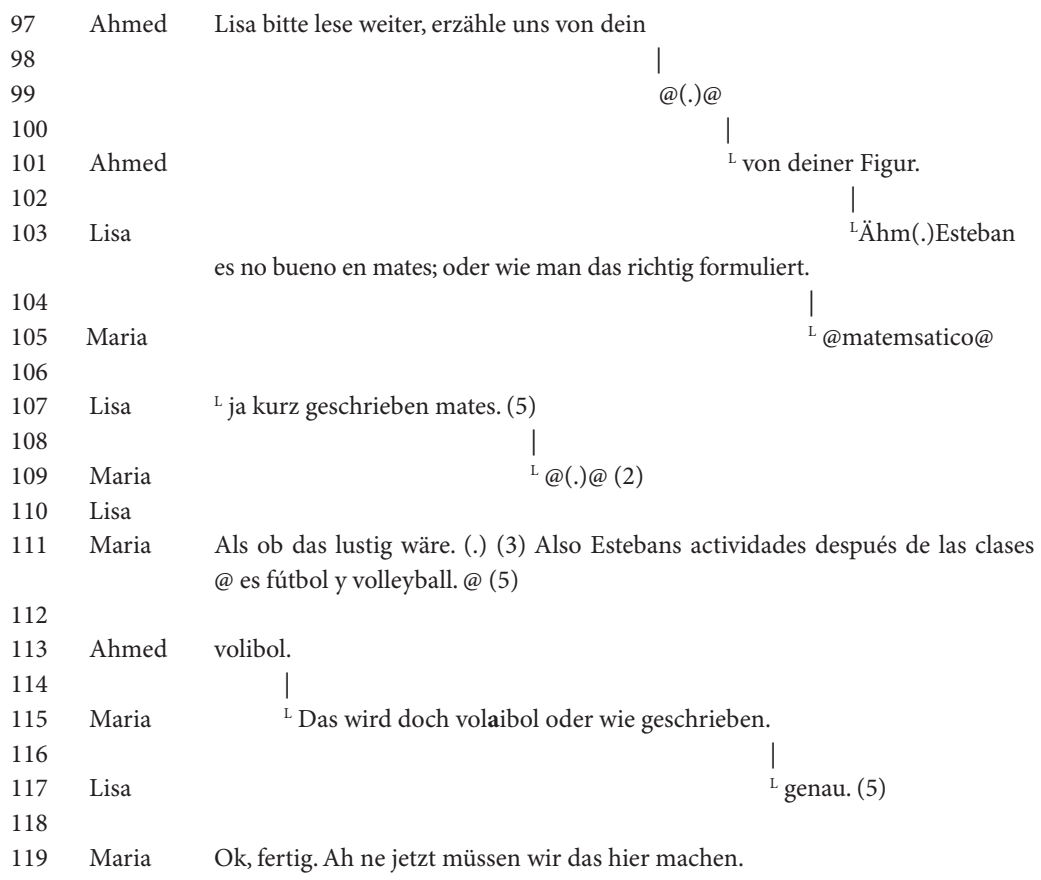

Bei Sprecherwechsel wird eine Leerzeile eingefügt. Przyborski bemerkt dazu (2004: 331), dass die Leerzeile die Lesbarkeit verbessert. In Sequenzen, in denen zwei oder mehr Personen zugleich sprechen, gewinnt man mehr Platz und in Sequenzen, in denen eine Person sehr lange ohne die Beteiligung der anderen spricht, wird Platz gespart. Hinzuzufügen wäre noch, dass ein senkrechter Balken die genaue Position der Überlappung markiert. 
Ein Problem kann die Transkription der Fremdsprache darstellen. Die Aussprache eines bestimmten Wortes kann Anlass für eine Interaktion sein. Daher sollte die Transkription die Graphie der fremdsprachigen Redeanteile prinzipiell normsprachlich korrekt wiedergeben; an Stellen jedoch, die Anlass für Kommentare geben, ist die besondere Aussprache phonetisch zu markieren. In der obigen Episode etwa ist dies in den Zeilen 112 bis 115 der Fall (volleyball, volibol, volaibol). Auf diese Stellen ist in der reflektierenden Interpretation gegebenenfalls näher einzugehen.

Ob tatsächlich die gesamte Unterrichtsstunde bzw. das gesamte Gruppengespräch/Interview oder nur einzelne Passagen transkribiert werden, hängt von der Erfahrung des Forschenden, seinem Erkenntnisinteresse und seinen zeitlichen Ressourcen ab. Gerade wenig erfahrene Forschende schärfen ihren Blick für die Relevanz bestimmter Passagen durch die Arbeit an der Transkription, was dafür spricht, sie selbst durchzuführen. Sie ist jedoch sehr zeitaufwändig und kann dadurch forschungsökonomische Zwänge provozieren. Mit der Zeit entsteht mehr und mehr Routine, sowohl was das Transkribieren selbst angeht als auch die Auswahl bestimmter Episoden für die Transkription und bestimmter Sequenzen für die reflektierende Interpretation. Diese Auswahlentscheidungen betreffen eine der Hauptfunktionen der sog. formulierenden Interpretation.

\subsubsection{Formulierende Interpretation}

Der nächste Arbeitsschritt der Interpretation führt von der Transkription zur formulierenden Interpretation. Bei ihr geht es darum, den transkribierten Text thematisch zu gliedern und zu reformulieren und dabei die Passagen zu identifizieren, die anschließend einer vertieften Interpretation unterzogen werden sollen. Jede durch Lehr-/Lernziele strukturierte Unterrichtsstunde ist wie auch jedes andere Gespräch oder Interview durch eine Abfolge von Themen und Subthemen gekennzeichnet, die vom Forscher zunächst einmal benannt und sortiert werden müssen, um einen Überblick zu gewinnen. Dabei mag sich herausstellen, dass bestimmte Themen eng am Thema der Unterrichtsstunde oder der Fragestellung des Forschers liegen, andere dagegen weiter entfernt scheinen. Dies heißt jedoch nicht, dass diese weniger interessant für die tiefergehende Interpretation sind als jene. In der Kumulation bestimmter Themen oder Subthemen kann ein Indiz für die Relevanz des Themas innerhalb einer Personengruppe liegen ebenso wie in der scheinbaren Singularität oder Zusammenhanglosigkeit bestimmter Themen. Sie sollten Forschende im Gegenteil aufmerksam machen. Ein weiteres ganz entscheidendes Auswahlkriterium im Hinblick auf die reflektierende Interpretation ist die interaktive und metaphorische Dichte bestimmter Passagen, da sich 
in ihr eine gesteigerte emotionale Beteiligung äußert. Dennoch sind gerade im schulischen Fremdsprachenunterricht Routine- oder Standardabläufe, wie z.B. Reihenübungen mit scheinbar geringer emotionaler Beteiligung, nicht automatisch von der Interpretation auszuschließen, da es selbstverständlich auch dort zu Interaktionen kommt, die unter einer bestimmten Fragestellung, wie z.B. das Feedbackverhalten von Mitschülern und Lehrpersonen, interessant sein können.

Dem Forscher obliegt es, aus der Fülle des Materials eine begründete Auswahl an vertieft $\mathrm{zu}$ interpretierenden Passagen zu treffen. Die formulierende Inter-

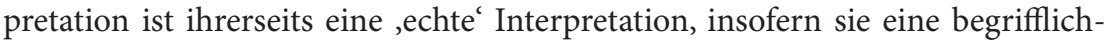
theoretische Abstraktion beinhaltet, die die Beforschten nicht vollziehen: Der Forschende formuliert Oberbegriffe und Themen für Diskurspassagen, er fasst Abschnitte zusammen oder paraphrasiert sie und er notiert, wer die Interaktion einleitete und wer an ihr beteiligt war. Es hat sich bewährt, innerhalb der formulierenden Interpretation mit einer Zwei-Ebenen-Struktur zu arbeiten, nämlich der Ebene der listenartigen Themennennung und der Ebene der Inhaltsangaben. Folgende Tabelle zeigt die Ebene der Themenlistung am Beispiel einer Seminarevaluation:

Tabelle 3: Listenartige thematische Gliederung einer Gruppendiskussion im Anschluss an ein fachdidaktisches Seminar

\begin{tabular}{|ll|}
\hline $003-008$ & Praxisbezug, Unterrichtserprobung \\
$010-018$ & Theoriedefizit \\
$019-031$ & Praxisbezug, Unterrichtserprobung, Theoriedefizit \\
$032-063$ & Probleme bei der Klassenfindung \\
$064-073$ & Roter Faden des Seminars sehr deutlich erkennbar \\
$074-075$ & Ausreichend Zeit für die Aufgabenentwicklung \\
$076-077$ & Soll- und Ist-Vergleich \\
$078-082$ & Kreativität entwickeln \\
$083-087$ & Gruppenarbeit: Interaktivität \\
$088-094$ & Gruppenarbeit: Arbeitsökonomie \\
$095-106$ & Gruppenarbeit: soziale Interaktion \\
$107-110$ & Gruppenarbeit: Gruppengröße \\
$111-132$ & Gruppenarbeit: Hausarbeit \\
$133-146$ & Gruppenarbeit: Betreuung während der Gruppenphase \\
$147-173$ & Gruppenarbeit: soziale Interaktion \\
$174-214$ & Probleme bei der Schulfindung \\
$215-226$ & Häufiger Dozentenwechsel \\
$227-233$ & Probleme beim Noteneintrag \\
\hline
\end{tabular}




\begin{tabular}{|ll|}
\hline $234-241$ & Vorteil der Aufgabenerprobung in einer Klasse \\
$242-258$ & Unterschied zu den schulpraktischen Studien \\
\hline $259-272$ & Gutes Kursklima; Kursbeginn 08.00 Uhr \\
\hline $273-282$ & Theoretischer Input \\
\hline $283-293$ & Gruppenarbeit: Länge der Gruppenarbeitsphase; Kursbeginn 08.00 Uhr \\
$294-314$ & Umgang mit Abwesenheiten; menschliche Seite \\
\hline $315-326$ & Schwierigkeit die theoretischen Texte zu verstehen \\
$327-331$ & Arbeitssprache Spanisch \\
$332-333$ & Schwierigkeit die theoretischen Texte zu verstehen \\
$334-341$ & Oberflächlichkeit in früheren Seminaren \\
$342-348$ & Deutsche oder spanische theoretische Texte \\
$349-354$ & Bestimmte theoretische Fragen unbeantwortet geblieben \\
$355-361$ & Schwierigkeit die theoretischen Texte zu verstehen \\
$362-376$ & Wunsch nach Ausgabe der PPT durch den Dozenten \\
\hline
\end{tabular}

Bei diesem Beispiel einer im Anschluss an ein fachdidaktisches Seminar durchgeführten Gruppendiskussion sind die beiden meist genannten Themen farblich hervorgehoben, nämlich der Theorie-Praxis-Bezug des Seminars sowie die Herausforderungen der Gruppenarbeit.

Die Themenliste erlaubt es, auf einen Blick die gesamte Unterrichtsstunde bzw. das gesamte Gespräch im Blick zu halten, während Inhaltsangaben eine Auswahl der vertieft zu interpretierenden Passagen erleichtern. Die formulierende Interpretation verlässt jedoch in keinem Falle das Relevanzsystem der Beteiligten. Der Forscher paraphrasiert oder fasst lediglich zusammen, was die Beteiligten äußern. Das folgende Beispiel zeigt die Ebene der Inhaltsangabe und illustriert die Art der Paraphrasierung:

Transkriptauszug 075-088:

Leonie:

75 Ähmm dann die Erprobung der Aufgabe um das einfach nochmal also ähm ja;

76 „Soll und Ist“ zu vergleichen also das fand ich auch echt ähhh sehr gut und 77 ähm ich find das auch gut dass wir das im Anschluss vorstellen weil dann 78 kriegt man auch immer neue Anregungen (.) ähm ne was ham die andern 79 gemacht und (1) ich find ich glaube dieses Seminar kann dazu beitragen dass 80 man halt auch ein bisschen kreativer wird und dann vielleicht auch ein 81 bisschen ähhmm sich nicht von den Lehrbüchern komplett verabschiedet 82 aber einfach ein bisschen auch nach links und rechts guckt und sich überlegt 83 äh was könnte man so machen (.) und ähm (1) ja ähm ja ich finds auch gut im 84 Prinzip dass es inner Gruppe läuft aber ähmm da muss ich sagen also es ist ja 
85 (1) halt immer so'n Gruppen (1) das äh, immer mal irgendwie @jemand was

86 vergisst die Texte mitzubringen oder so @ ähm aber das ist ja; das ist halt ein

87 generelles Problem; das kann man nicht ändern und ich denk mal wenn man

88 in ner Gruppe interagiert kommen auch ganz nette Ergebnisse raus (.)

Eine formulierende Interpretation könnte diesen Redeabschnitt folgendermaßen zusammenfassen:

Leonie äußert zwei Vorteile der neuen Seminarform: a) dass die Aufgabenerprobung die Unterschiede zwischen Aufgabenplanung und Realität der Umsetzung deutlich werden lässt und b) dass sie die Studierenden in den Stand versetzt, durch die eigenen Ideen erste Ansätze von Autonomie zu erwerben. Zur Gruppenarbeit äußert sie trotz zu erwartender Unzuverlässigkeiten einzelner Teilnehmer im Ergebnis eine positive Bewertung.

Der nächste Interpretationsschritt - das Wie der Äußerungen - bleibt der reflektierenden Interpretation vorbehalten. Diese Selbstdisziplin bei der Beachtung des Was von Äußerungen ist eine große Hilfe bei der Strukturierung des Textes, denn auf diese Weise kann die Gefahr minimiert werden, den Text als Ganzes aus den Augen zu verlieren. Sie kann eine Hilfe darstellen, die interne Verweisstruktur eines Textes (Indexikalität, s. Kap. 2.1) herauszuarbeiten.

Gleichzeitig - und dies ist ein weiteres zentrales Anliegen beim Arbeitsschritt der formulierenden Interpretation - lernt der Forschende auf diese Weise, sich von der Sprache der Beforschten zu lösen und sie begrifflich zu abstrahieren. Damit und mit der Trennung der Analyseschritte ist ein genuines Gütekriterium der rekonstruktiven Fremdsprachenforschung mit der Dokumentarischen Methode erfüllt, da beides dazu beiträgt, die Validität der Ergebnisse zu sichern. Rekonstruktiv ermittelte Ergebnisse sind valide, wenn sie einer methodischen Kontrolle unterliegen, d.h. wenn „eine Kontrolle über die Unterschiede der Sprache von Forschenden und Erforschten, über die Differenzen ihrer Interpretationsrahmen, ihrer Relevanzsysteme“ (Bohnsack 2014: 22) erfolgt. In der formulierenden Interpretation löst sich der Forscher zwar nicht von den Relevanzsystemen der Beforschten, doch er löst sich von ihrer Sprache, wie das obige Beispiel zeigt. Aus diesem Grunde kann eine Analyse des Wie bei offenen Analyseverfahren erst geschehen, nachdem eine klare Trennung zum Was hergestellt wurde.

\subsubsection{Reflektierende Interpretation}

Wie in Kapitel 1 ausgeführt, geht es bei der Erforschung des Fremdsprachenunterrichts mit Hilfe der Dokumentarischen Methode um vier Sinnebenen (intendierter Sinn, kommunikativer Sinn, dokumentarischer Sinn und lernersprachlicher Sinn). In der reflektierenden Interpretation bearbeitet der Forscher 
die dokumentarische Sinnebene und die wechselseitige Verschränktheit von dokumentarischem Sinn und lernersprachlichem Sinn bezogen auf ein gemeinsames Dittes (tertium comparationis). Dieser Analyseschritt stellt den Kern der Interpretation dar. Er erfolgt - nachdem die reflektierend zu interpretierenden Passagen identifiziert wurden - in der Konzentration auf bestimmte deutlich zu Tage tretende Merkmale, wie:

- Metaphern (von Bohnsack auch als „Fokussierungsmetaphern“ bezeichnet)

- Passagen besonderer interaktiver Dichte, meist markiert durch überlappende Redebeiträge, Auflachen oder Kichern etc.

- Passagen besonderer emotionaler Dichte, markiert z.B. durch heftige verbale Reaktionen (z.B. Kraftausdrücke), betretenes Schweigen, Stottern, ironische Kommentare, etc.

Mit der Identifikation solcher Textpassagen beginnt die Feinrekonstruktion des Diskursverlaufs. Dabei zielen Forschende darauf ab, über das Wie der Äußerungen zu einem Gerüst der positiven und negativen Positionierungen („Gegenhorizonte“) zu gelangen, das im Laufe der Interpretationsarbeit immer engmaschiger wird und durch das Herausarbeiten der Orientierungsrahmen und ihrer Indexikalität schließlich die hinter dem Diskurs liegenden sozial geteilten Erfahrungsräume (sinngenetische Interpretation, s. u. 3.1.5) sichtbar werden lassen.

Voraussetzung zur Erfassung des dokumentarischen Sinns ist die Rekonstruktion der formalen Diskursstruktur. Diese befindet sich in einer zirkulären Wechselbeziehung zum semantischen Gehalt der Äußerungen. Charakteristisch dafür ist der in der Diskursentwicklung eingelagerte Dreischritt aus Proposition, Ausführung und Konklusion:

Tabelle 4: Modi der Diskursorganisation in Anlehung an Przyborski (2004)

\begin{tabular}{|c|c|c|}
\hline Proposition & Vergleichshorizont & Modus der Diskursbewegung \\
\hline 1. Proposition & Positiver Vergleichshorizont & Initiierung \\
\hline \multirow{3}{*}{ 2. Ausführung } & Positiver Gegenhorizont & Elaboration \\
& & $\begin{array}{c}\text { Differenzierung } \\
\text { Validierung } \\
\end{array}$ \\
\cline { 2 - 3 } & Regativer Gegenhorizont & Antifierung \\
& & Opposition \\
& & Divergenz \\
\hline
\end{tabular}




\begin{tabular}{|c|c|c|}
\hline Proposition & Vergleichshorizont & Modus der Diskursbewegung \\
\hline \multirow[t]{3}{*}{ 3. Konklusion } & Thematische Konklusion & $\begin{array}{l}\text { Positive Bewertung } \\
\text { Meinungssynthese }\end{array}$ \\
\hline & Rituelle Konklusion & $\begin{array}{l}\text { Themenwechsel } \\
\text { Formalsynthese } \\
\text { Themenabweisung }\end{array}$ \\
\hline & Metakommunikation & \\
\hline
\end{tabular}

Die Proposition eröffnet ein Thema gegebenenfalls durch eine Aussage, die die Richtung einer Orientierung vorgibt. Das Thema wird nun von den Gesprächsteilnehmern weiterbearbeitet, und auf die Aussage (Vergleichshorizont) erwidert ein Gesprächspartner mit einem positiven oder einem negativen Gegenhorizont. Der Gesprächspartner bleibt dem durch die Proposition vorgegebenen Rahmen treu, oder aber er verlässt den gemeinsamen Rahmen. Dafür stehen verschiedene Modi zur Verfügung: für eine übereinstimmende Rahmung die inkludierenden Modi der Elaboration, der Differenzierung, der Validierung, der Ratifizierung oder der Antithese, für eine abweichende Rahmung die exkludierenden Modi der Opposition oder der Divergenz. Nach Asbrand (2011: 8) „wird das Thema für die Gruppe desto relevanter eingeschätzt, je mehr Gruppenmitglieder sich an [seiner] Elaboration beteiligen."

Die Diskursbewegung wird wiederum auf drei mögliche Arten abgeschlossen,

- entweder thematisch durch eine positive Bewertung („Stimmt“) oder eine Meinungssynthese,

- rituell durch einen Themenwechsel („OK, fertig. Ah ne jetzt müssen wir das hier machen"), eine Themenabweisung oder eine formale (nicht inhaltliche) Synthese

- und als dritte Möglichkeit durch eine Äußerung im Modus der Metakommunikation. Ein Beispiel für die Analyse einer Diskursbewegung findet sich in Kap. 2.1, weitere Beispiele in Kapitel 4.1.

Das Besondere an fremdsprachenunterrichtlichen Diskursen wie auch an ausserunterrichtlichen Diskursen, bei denen sich Sprecher verschiedener Herkunftssprachen unter Verwendung einer lingua franca verständigen, ist der Gebrauch der fremden Sprache(n). Dieser kann als Ausdruck beobachtbarer Performanz oder als Dimension der Sprachbewusstheit mit in die Interpretation einbezogen werden. Dabei bewirkt freilich nicht die Verwendung der fremden Sprache an sich bereits das Vorhandensein einer konjunktiven Wissensebene, sondern die mit ihr verbundenen Formen der Sprachbewusstheit. Diese manifestiert sich 
z.B. im Wissen um systematische Regelhaftigkeit, um Übereinstimmung oder Abweichung von der Norm, um Prozesswissen in der Verwendung der Fremdsprache, um den manipulativen Charakter bestimmter sprachlicher Äußerungen und um die Wirkung bestimmter sprachlicher Mittel (vgl. Vollmer 2016: 202 ff.).

In der reflektierenden Interpretation arbeitet der Forscher $u$. a. die wechselseitige Verschränktheit von dokumentarischem Sinn und lernersprachlichem Sinn bezogen auf ein gemeinsames Dittes (tertium comparationis) heraus. Die Parallelität der beiden Sinnebenen lässt sich in den Forschungstraditionen der soziokulturellen fremdsprachenbezogenen Diskursanalyse (vgl. Lantolf \& Poehner 2008: 48) ebenso wie in den Veröffentlichungen zur Dokumentarischen Methode auch auf der Ebene der Mikroanalyseschritte erkennen:

Tabelle 5: Lernersprachliche turns und soziale Diskursorganisation

\section{Fremdsprachenunterrichtliche Konstruktion als Diskursbewegung}

Entwicklungsbewegung (turn)

der Lernersprache in der ZPD

(soziokulturelle Theorie)

1) Orientierung

2) Ausführung

3) Evaluation (Kontrolle)
Soziale Diskursorganisation

(Dokumentarische Methode)

1) Proposition

2) Ausführung

3) Konklusion

Die Entwicklung der Lernersprache in der zone of proximal development wird in der soziokulturellen Sprachenforschung als Diskursbewegungen (turns, Sacks et al 1974) bestehend aus Orientierung, Ausführung und Evaluation (auch: Kontrolle) (Gal'perin, nach Lantolf \& Poehner 2008: ibd.) verstanden, während die rekonstruktive Sozialforschung auf sozial geteilte Orientierungen im Dreischritt Proposition, Ausführung und Konklusion (Bohnsack 2014: 127; Przyborski 2002: 62-76) fokussiert. Wichtig ist, dass der Forscher im Zuge der reflektierenden Interpretation stets den Vergleichspunkt, das tertium comparationis, im Auge behält. In der Passage „Esteban“ (s. oben 3.1.1) geht es um die diskursive Entwicklung einer Gruppenarbeit im beginnenden Spanischunterricht und darin u. a. um die Verwendung bestimmter Wortverbindungen (z.B. ser bueno en mates). Beides kann mit weiteren Episoden im selben Fall verglichen werden, aber auch mit entsprechenden Wortverbindungen in weiteren beobachteten Gruppenarbeiten. Im Falle der weiter oben (s. 3.1.2) vorgestellten Passage aus einer Seminarevaluation geht es dagegen u. a. um das Thema „Theorie-PraxisVerhältnis in fachdidaktischen Seminaren", und fallintern kann nun verglichen werden, wie dieses Thema in Rede und Gegenrede bzw. in Vergleichshorizonten 
bearbeitet wird. Ein Nebenthema dieses tertium comparationis, das verhandelt wird, ist die Gruppenarbeit. Es findet eine Verständigung über die Vor- und Nachteile von Gruppenarbeit statt. Unter 3.1.1 taucht die Gruppenarbeit ebenfalls als tertium comparationis auf (Gruppe Blau, Passage „Esteban“), diesmal jedoch analysiert der Forscher nicht die Verständigung über Gruppenarbeit, also die Metakommunikation, sondern die diskursiv-praktische Entwicklung einer Gruppenarbeit im konjunktiven Erfahrungsraum des Unterrichts. Hier hat - wie soeben gezeigt - auch der Vergleich der performativen Verwendung der Lernersprache und der mit ihr verbundenen Sprachbewusstheit seinen Platz. Im Gebrauch der Lernersprache äußert sich die aus der Psycholinguistik übernommene intraindividuelle Interaktion von Top-down- und Bottum-up-Prozessen (Oberflächenverstehen sowie Verknüpfung mit Struktur- und Weltwissen), die Interaktion von Konzeptualisierer, Formulator und Artikulator mit dem Monitor (De Bot 1992; Levelt 1989) sowie die soziale Interaktion unter den Gruppenmitgliedern. Letztere kann mit anderen Passagen derselben Gruppe sowie mit Episoden anderer Gruppen derselben Klasse im Sinne des minimalen und maximalen Kontrasts nach Glaser und Strauss (1967) verglichen werden und führt zur sinngenetischen Typenbildung (s. u. 3.1.5).

\subsubsection{Fallbeschreibung}

Die Fallbeschreibung dient der Vermittlung der Interpretationsergebnisse an das Fachpublikum. In dieser Hinsicht ist offensichtlich, dass es sich bei diesem Arbeitsschritt nicht um ein einfaches Zusammenschreiben der in der reflektierenden Interpretation vorformulierten Bausteine handelt, sondern um eine grundsätzliche Reorganisation von Interpretationsergebnissen. In diesem Arbeitsschritt bekommt der Fall als solcher seine Gesamtgestalt, denn er wird verbal konturiert und zu anderen homologen Fällen in Bezug gesetzt. Zugleich erzählt der Forscher die Geschichte des Falls sowie die Geschichte aller untersuchten Fälle. Das heißt, er fügt die verschiedenen Analyseergebnisse zu einem kohärenten Text zusammen und macht sie für Außenstehende nachvollziehbar. Die Textsorte Fallbeschreibung besitzt eigene Strukturmerkmale, nämlich eine hohe Textkohärenz (z.B. in Form von Gliederungsmerkmalen, Übergangsmarkierungen und dem deutlich erkennbaren, roten Faden') sowie eine eigene Narrativik. Diese hängt nicht nur vom Schreibstil des Forschers ab, sondern auch von den Merkmalen der beobachteten Fälle. Die Beschreibung eines ,Unterrichtsfalls' sieht bereits auf Grund der zitierten Diskursstruktur anders aus als die Beschreibung eines narrativen Interviews zur individuellen Sprachlernbio-

grafie einer Spanischstudierenden. Ein ,Fall' ist mithin immer auch eine erzählte Geschichte; sie bleibt jedoch den empirisch ermittelten Ergebnissen treu. 
In diesem Zusammenhang stellt sich die theoretisch vorab zu klärende Frage, was überhaupt als, Fall' bezeichnet wird bzw. welche Strukturmerkmale ein Ereignis zu einem Fall machen. Bereits in Kapitel 2.1 sowie weiter oben in diesem Kapitel wurde auf die Rolle des tertium comparationis, des zu vergleichenden Dritten, für die komparative Analyse hingewiesen. Daraus ergibt sich eine erste funktionale Bestimmung des Fallbegriffs: Fälle sind diejenigen Sequenzen bzw. Episoden, in denen sich das zu vergleichende Dritte manifestiert. Diese erste Bestimmung wäre sodann durch spezifische organisatorische Rahmungen des Unterrichts zu erweitern. In der Untersuchung zur Umsetzung einer komplexen Lernaufgabe zum Sprechen (Tesch 2010) wurden beispielsweise plenare Metagespräche (Evaluationsphasen) wie auch Arbeitsgespräche im Plenum und in Gruppen- und Partnerarbeit für die Analyse ausgewählt. Die Fallauswahl auf der Ebene einer bestimmten Klasse wurde dann durch eine Fallauswahl bei einer anderen Klasse und schließlich einer anderen Schule in einem anderen Bundesland ergänzt. Genau genommen sind folgende Strukturebenen für Fälle und für die Fallauswahl (sampling) zu unterscheiden:

Tabelle 6: Strukturebenen für die Klassifizierung von Fällen

\begin{tabular}{|l|l|}
\hline Strukturebene & Spezifizierung \\
\hline Bundesland & BL 1, BL 2, .. BL 16 \\
Schulform & $\begin{array}{l}\text { Gymnasium, Mittelschule, Grundschule, Freie Schule, } \\
\ldots\end{array}$ \\
Einzugsbereich & Großstadt, Kleinstadt, Land \\
Soziales Umfeld & $\begin{array}{l}\text { Bürgerliches Umfeld, Arbeiterumfeld, } \\
\text { migrationsgeprägtes Umfeld, ... }\end{array}$ \\
Klasse: Jahrgangsstufe & JS 1, Js 2, .. JS 13 \\
Klasse: Sprachenfolge & D+E+F+S, D+E+L+F, ... \\
Geschlechterzusammensetzung & Anzahl männlich, Anzahl weiblich \\
Migrationshintergrund & $\begin{array}{l}\text { Anzahl und Herkunft der Schüler mit } \\
\text { Migrationshintergrund }\end{array}$ \\
Sozialform & $\begin{array}{l}\text { Plenum, Gruppenarbeit, Partnerarbeit, Einzelarbeit } \\
\text { Methodische Großform }\end{array}$ \\
Lernperspektive & $\begin{array}{l}\text { Lehrwerksarbeit, Projektarbeit, freie Lektürearbeit, ... } \\
\text { Individuelle Selbstwirksamkeitserwartung, } \\
\text { Progressionserwartung, Peerbeziehungen, ... }\end{array}$ \\
Lehrperspektive & $\begin{array}{l}\text { Instruktion, Individualisierung, inhaltlicher } \\
\text { Schwerpunkt ... } \\
\text { Individuelle } \text { zones of proximal development }\end{array}$ \\
Individuelle Lernersprachen &
\end{tabular}


Ein unterrichtlicher Fall kann demnach institutionell durch ein Bundesland, eine Schulform, eine Schule oder eine Klasse bzw. Lerngruppe repräsentiert sein. Personell kann der Fall durch eine Kleingruppe, das Klassenplenum oder auch eine Einzelperson repräsentiert sein. Und konzeptuell kann er durch eine unterrichtliche Sozialform, eine methodische Großform, einen Lernaspekt, einen Lehraspekt und durch individuelle lernersprachliche Entwicklungszonen repräsentiert sein. Aus den untersuchten Kombinationen ergeben sich bestimmte Beziehungen, deren Charakter in die Fallbeschreibung einzubeziehen und ggf. in Form einer Mehrebenenanalyse (Nohl 2013) aufzuarbeiten ist. Mit anderen Worten, jeder Einzelfall ist eingebettet in eine strukturell betrachtet globalere bzw. hierarchiehöhere Struktur, er kann ebenso auch hierarchieniedrigere Strukturen umfassen, und er birgt überdies immer auch Überschneidungen mit anderen Fallstrukturen auf derselben Hierachieebene. Der Fall „Esteban“ (s.o. 3.2.1) beispielsweise birgt Strukturelemente folgender hierarchischer Vergleichsebenen: Sozialform (Gruppenarbeit bei Gruppe A im Vergleich zu Gruppe B, Gruppenarbeit generell im Vergleich zu Plenararbeit), Lernersprachen (in den verschiedenen individuellen zones of proximal development innerhalb des Niveau A2), Gruppenzusammensetzung (geschlechtergemischt im Vergleich zu geschlechterhomogen). Er birgt aber auch Strukturelemente der hierarchieparallelen Ebenen Peer-Beziehungen, Aufgabenbearbeitungskonzept, gewohnter Unterricht. ${ }^{22}$

Die Fallbeschreibung bezieht nun inhaltlich alle soeben genannten Strukturebenen ein und vollzieht auf der textstrukturellen Ebene alle vorhergehenden Arbeitsschritte nach und fasst sie zusammen. Sie spiegelt die thematische Gliederung des Falls, greift dabei Elemente der formulierenden Interpretation auf und fokussiert auf die Orientierungsrahmen einzelner analysierter Passagen. Der folgende Auszug verdeutlicht einige der genannten Strukturmerkmale der unteren Strukturebenen. Er stammt aus Tesch 2010 und fokussiert auf die Lernperspektive (individuelle Selbstwirksamkeitserwartung und Erwartungen an den Fremdsprachenunterricht). ${ }^{23}$

\section{Sequenz 6: „Ja, ich find einfach, dass wir zu wenig Französisch sprechen.“}

Zum Abschluss des Kapitels „Der gewohnte Unterricht“ kommen noch einmal Schüler der Klasse $10 \mathrm{~b}$ zu Wort, die die Thematik der unzureichenden

$22 \mathrm{Zu}$ den methodologischen Problemen beim Vergleich von Fällen über mehrere Hierarchieebenen hinweg, z.B. einer Gruppenarbeit in Schule A im Vergleich zu einer Gruppenarbeit in Schule B, s. Nohl (2013, 105 ff.)

23 Ein Beispiel einer Fallbeschreibung auf abstrakterer Ebene findet sich in Kapitel 4.1. Dort liegt der Fokus auf der Sozialform (Gruppenarbeit). 
Verwendung des Französischen im Unterrichtsgespräch ansprechen. Die Unterrichtsstunde schließt sich an die Posterpräsentation nach Modul 5 an, das den inhaltlichen Höhepunkt der Bearbeitung von pir@tes du net in Klasse 10 b bildete. Der Unterrichtende führt mit der Klasse ein Unterrichtsgespräch auf Englisch über das Herunterladen von Musik und das Einkaufen im Internet. Anschließend bespricht er mit der Klasse die Unterschiede zwischen Englisch als Unterrichtssprache und Französisch.

Schulte ${ }^{\mathrm{L}}$ Warum, warum?

Micha Mir kommt es irgendwie einfacher vor. ${ }^{\circ}$ Ich weiß auch nicht warum, aber ${ }^{\circ}$ wenn ich englisch rede, dann kommt es leichter. Mir fallen, fallen dann auch schneller die Wörter ein als im Französischen. Da muss man immer überlegen, ob man jetzt ein Verb konjugieren muss

Schulte $\mathrm{L}_{\mathrm{Ehem}}$.

Micha $\quad{ }^{\circ}()^{\circ}$.

Schulte Du meinst weil sowieso jeder da draußen auf der Erde meint, er könne Englisch und, ähm, weil

Micha Englisch ist ja nicht einfacher, aber, mh, es richtig zu sprechen ist bestimmt (.) viel schwieriger, aber, $\mathrm{mh}$, ich weiß nicht (.)

(3768-3779)

Micha äußert, dass er sich im Englischen sicherer fühlt. Auf Nachfrage des Unterrichtenden begründet er diese erste spontane Empfindung mit Hilfe weiterer präziserer Beschreibungen und entwickelt einen Katalog seiner Schwierigkeiten mit der französischen Sprache im Vergleich zum Englischen (3770 - 3773):

- Englisch sei „irgendwie“ einfacher

- Englisch „komme leichter“

- Im Englischen fielen ihm schneller die Wörter ein

- Im Französischen müsse man immer überlegen, ob man jetzt ein Verb konjugieren müsse.

Michas Gegenhorizont impliziert die Rahmung einer generell hohen Selbstwirksamkeitserwartung [bezogen auf das Englische] und damit eine gewisse Resignation im Hinblick auf das Französische. Bei Micha steht der Zwang zur Korrektheit über der Flüssigkeit, und die Flüssigkeit leidet besonders unter der mangelnden Verfügbarkeit der Lexik und der besonderen Schwierigkeit der Verbkonjugation. Es stellt sich die Frage, wie Micha zu dieser Wahrnehmung kommt bzw. inwieweit der vorausgegangene Unterricht zu dieser Wahrnehmung geführt hat. Aus der Sicht Michas ist es demotivierend zu sehen, dass er über Englisch in gewissem Grad verfügt, über Französisch jedoch nicht. [Carla validiert Michas Sicht].

(Tesch 2010, 201 f.)

Zur Erläuterung des Fallauszugs: Er trägt eine aussagekräftige Überschrift, die einem Zitat entnommen wurde, das weiter unten in der Fallbeschreibung aufgeführt wurde. Die Episode wird zunächst in den Zusammenhang des Kapitels 
und in diesem Falle in den Kontext der Unterrichtsbeobachtungen eingeordnet. Es handelte sich hier um die Beobachtung der Bearbeitung einer Lernaufgabe über mehrere Unterrichtsstunden hinweg, so dass der Leser erfahren sollte, um welche Stunde, welches Stundenziel und welches, verhandelte' Thema oder Subthema es sich handelte. Als nächstes folgt das Transkript einer Passage, die das Subthema illustriert; hier lautet das Subthema „Unterschiede zwischen Englisch und Französisch als Unterrichtssprache". Auf das Transkript folgt eine kurze Zusammenfassung des Inhalts, bevor die in der Passage artikulierte Aussage gedeutet wird. Carlas Aussage bestätigt diesen Gegenhorizont im Sinne des fallinternen Vergleichs.

Die Fallbeschreibung greift, wie man sieht, auf die Ergebnisse der reflektierenden Interpretation zurück, allerdings verzichtet sie auf die detaillierte Wiedergabe der Diskursbewegungen. Transkriptauszüge dienen der Illustration. Dafür arbeitet der Forscher in der Fallbeschreibung klar das sozial geteilte Wissen der Verständigung, - die sogenannten Rahmenorientierungen - heraus und stützt sich dabei auf die Vergleichshorizonte zum tertium comparationis. An sie kann nun der letzte Arbeitsschritt anknüpfen, die Typenbildung.

\subsubsection{Typenbildung}

Theoriebildung auf Grundlage empirisch generierter Typiken ist das Ziel der Dokumentarischen Analyse. Aber worauf beruhen die Typiken? Bohnsack (2014: 145) schreibt dazu: „Der Kontrast in der Gemeinsamkeit ist fundamentales Prinzip der Generierung einzelner Typiken und ist zugleich die Klammer, die eine ganze Typologie zusammenhält“. Diese Bestimmung geht auf Glaser \& Strauss (1967, s. u.) zurück und meint die maximalen und minimalen Abweichungen in der diskursiven Bearbeitung bestimmter Themen bzw. Vergleichspunkte innerhalb eines konjunktiven (sozial geteilten) Erfahrungsraums. In der bereits zitierten Studie (Tesch 2010) konnte beispielsweise das komplexe Typengeflecht bei der Bearbeitung ein- und derselben komplexen Französisch-Lernaufgabe (= tertium comparationis) im Rahmen einer dreiwöchigen Unterrichtssequenz in einer neunten und zwei zehnten Klassen herausgearbeitet werden (ibd. Kap. 2.2). In dieser Studie konnten auf Seiten der Schüler der Einfluss spezifischer Lernhandlungskonzepte (z.B. Orientierung an den Peers oder Orientierung an persönlicher Progression) aber auch der Einfluss der schlichten Macht der Gewohnheit sowie individueller Selbstwirksamkeitserwartungen und aufgabenbezogener Bearbeitungsmuster nachgewiesen werden. Auf Seiten der Lehrpersonen zeigten sich im Fallvergleich sehr ähnliche professionelle Orientierungen, z.B. durchgängig ein 
kontrollierendes und eher instruktivistisch orientiertes Lehrkonzept, das durch Phasen bewusster gemeinsamer Sinnkonstruktion nuanciert wurde.

Für den Vorgang des Generierens einer Typik gibt es keine spezielle Technik. Forschende müssen die Befunde aus der reflektierenden Interpretation der verschiedenen Fälle interpretativ zusammenbringen und dabei Gemeinsamkeiten und Unterschiede erkennen. So entsteht zunächst eine „sinngenetische Typik“, d.h. eine Deutungshypothese bezogen auf verschiedene fallbezogene Rahmenorientierungen. Diese Typik stellt bereits eine Abstraktion von Rahmenorientierungen dar, die innerhalb der reflektierenden Interpretation durch minimale und maximale Kontrastierung herausgearbeitet werden können. Die Begriffe „minimale" und "maximale Kontraste" schließen an die constant comparative method in der Grounded Theory (Glaser \& Strauss 1969: 101 ff.; vgl. Nentwig-Gesemann 2007: 290 f.; Nohl 2013: 15-41) an. Ihre Anwendung als Analyseprinzip auf der sinngenetischen Ebene führt zur Bildung von Basiskategorien, die in der Dokumentarischen Methode generell als „Typus“ bezeichnet werden. Beispielsweise konnten bei der oben erwähnten Untersuchung der Umsetzung einer komplexen Lernaufgabe ambivalente Typiken unter den Lernenden ermittelt werden, was die Haltung zu lehrwerksunabhängigen Materialien betraf. Während eine Mehrheit diese auf Grund ihrer wahrgenommen höheren Authentizität begrüßte (s. u. Nino, Gina), lehnten einige wenige sie ab, da sie ein gleichmäßiges Voranschreiten der gesamten Lerngruppe behinderten (s. u. Miriam):

Nino

Ja also ich denk schon dass es neu war vor allem war neu dass halt wirklich die Texte ähm ich sach mal aus der Realität stammen. Im Buch das sind ja irgendwelche Geschichten die sich irgendwelche Schulbuchschreiber aus den Fingern gesogen haben aber hier hat man ja wirklich aus ähm aus Zeitschriften oder so Ausschnitte auch bei der Starakademie irgendwelche Blogeinträge oder so was halt schon realitätsbezogen ist.

Gina

Hm also ich weiß nicht da bei () bei dieser Unterrichtseinheit konnte man auch irgendwie so auch was lernen also nicht dass wir aus dem Englisch äh aus dem Französischbuch nichts lernen könnte aber das Französischbuch ist ein bisschen () gewöhnungsbedürftig. Ein bisschen mehr gewöhnungsbedürftig und ich weiß nicht wenn man da neben halt nem Text sitzt man liest und den dann schon halt auswendig kann und so und dann bei diesen Unterrichtsteilen ist halt immer Abwechslung dabei () und wenn man zum Beispiel nicht weiß was ein Modem ist weiß er das jetzt. (ibd.: 209)

Ein Vergleich mit den thematisch verwandten Episoden über alle drei Klassen hinweg zeigte, dass die Kritik am gewohnten Lehrmaterial eine zentrale 
Rahmenorientierung darstellte, die somit wiederum als typisch für die untersuchten Klassen betrachtet werden konnte. Die minimale Kontrastierung innerhalb ein und derselben Lerngruppe erlaubte es, die interne Homogenität (s. Nentwig-Gesemann 2007: 296) des Typus ,positive Einstellung zum gewohnten Lehrmaterial' zu prüfen. Es stellte sich heraus, dass dieser spezielle Typus nur eine geringe interne Homogenität aufwies. Dieselbe Person, die sich für die traditionellen Lehrmaterialien aussprach, hob gleichzeitig die Förderung der Gruppenarbeit in den neuen Materialien hervor:

Miriam

Also ich fand, mh, die Aufgaben so war diss (.) macht diss mehr Spaß weil man eben mehr in Gruppen zusammen gearbeitet hat. Was im Lehrbuch aber besser ist, da war zum Beispiel jetzt, was auch schon oft angesprochen wurde mit den Vokabeln diss da hatte dann jeder im Lehrbuch stehen die Vokabeln da sind es immer dieselben und bei den Aufgaben war es dann halt da hat der eine diss rausgesucht der nächste hatte wieder was ganz anderes also hätte man irgendwie die Vokabeln mehr (.) dass jeder dieselben Vokabeln hat und nicht jeder für sich da seine Vokabeln hat sozusagen.

(Tesch 2010: 189)

Die maximale Kontrastierung wiederum, also die Suche nach der maximalen Unterschiedlichkeit zur Haltung gegenüber den neuartigen Materialien, erlaubte es, die externe Heterogenität ${ }^{24}$ (Nentwig-Gesemann 2007: 296) des Typus zu prüfen. Im Ergebnis gab es zwar sehr differenzierte und heterogene Stellungnahmen zu neuen Lehrmaterialien, letztlich liefen sie jedoch mehrheitlich darauf hinaus, dass die Materialien selbst nur einen Teil des Lernerfolgs erklärten. Viel stärker fielen die auf die Materialien bezogenen Lehrerhandlungen ins Gewicht:

Saskia

Ja also was auch schon gesagt worden ist also was wirklich ungewöhnlich ist ist dass ähm dass es einfach auch wirklich auf die moderne Welt auch zugeschnitten war und dass einfach wir hatten mehr von den alltäglichen Themen nicht was einfach (.) grau gekaut im Buch steht und ähm was ich auch auch gut fand war das ähm ja mit den Diskussionen so gut das ist immer das was man sonst auch macht das denk ich deswegen war es bei uns auch schon ähnlich also wir machen im Buch teilweise also wir machen auch schon Diskussionen und so was jetzt natürlich nicht im Buch steht aber ich denk da ist unsre Lehrerin dann für verantwortlich. So was ähm so was find

24 Auch statistische Untersuchungen kennen Homogenitäts- bzw. Heterogenitätsüberprüfungen als „Model-fit“ mit Hilfe von Tests und geeigneten Kennzahlen. Einige Parallelitäten und Diskrepanzen zwischen standardisierten hypothesenprüfenden und interpretativen theoriegenerierenden Verfahren werden von Bohnsack (2014: 15 ff.) hinsichtlich der Gütekriterien Validität und Reliabilität im Sinne einer allgemeinen erkenntnistheoretischen Reflexion kritisch gewürdigt. 
ich halt auch gut aber ja ähm es kommt halt immer darauf an wie man damit umgeht also man kann keine perfekte Unterrichtsvorbereitung machen und dann erwarten dass da ein perfekter Unterricht raus kommt. Also es kommt immer noch ähm auf andere an.

(Tesch 2010: 210)

Erst im zweiten Schritt werden nun die Erfahrungsräume, in denen die einzelnen Orientierungsrahmen entstanden sind, ausgeleuchtet und auf soziale Bedingtheiten hin interpretiert. Dieser zweite Schritt in der Typenbildung wird "soziogenetische Typenbildung" genannt und führt nach der Zusammenschau aller Typiken zu einer geschachtelten Typologie. Im Falle der oben geschilderten Orientierungen an einer kritischen Haltung zu den traditionellen Lehrmaterialien spielen generell neben medienspezifischen Begründungen (z.B. mangelnde methodische Alternativen, ergebnisgeschlossene versus ergebnisoffene Arrangements) auch die organisationstypischen Bedingungen des Lehrmaterialeinsatzes in Schulen eine entscheidende Rolle. Dazu zählen der Lehrwerkszwang in der Sekundarstufe I, der Zwang zur Amortisierung einmal angeschaffter Lehrwerke über viele Jahre hinweg, der immer größer werdende didaktische Rückstand der Materialien, die hohe Wochenstundenbelastung der Unterrichtenden und die dadurch bedingte Notwendigkeit zur Zeitökonomie bei der Unterrichtsvorbereitung, die oft gering ausgeprägten methodischen Routinen bei Berufsanfängern, etc. Die organisationstypischen Bedingungen können im vorliegenden Fall aus den Daten selbst nur indirekt erschlossen werden. Dazu wäre eine Analyse der institutionell-organisatorischen Rahmenbedingungen erforderlich.

In der Regel treten die Kontraste deutlich hervor, jedoch bedarf es einer gewissen Analyseerfahrung, um aus vordergründigen Parallelitäten oder Kontrasten nicht falsche Schlüsse zu ziehen und einfach auf wissenschaftlich kanonisierte Schemata zurückzugreifen. Die Validierung der Ergebnisse in einer Forschergruppe bietet die Gewähr für eine gelingende Typenbildung und gilt daher als unerlässliche Voraussetzung für deren Validität.

Die Komplexität einer Typik wird von Bohnsack als Gradmesser für ihre Validität gesehen (s. Bohnsack 2014: 145):

Die Typenbildung ist also umso valider, je klarer am jeweiligen Fall auch andere Typiken aufgewiesen werden können, je umfassender der Fall innerhalb einer ganzen Typologie verortet werden kann. Anders ausgedrückt bedeutet dies, dass als Voraussetzung für eine valide Typenbildung die den Fall konstituierenden unterschiedlichen Erfahrungsräume, aus denen heraus die unterschiedlichen Typiken generiert werden, in ihrer Abgrenzung voneinander wie in ihrem Bezug aufeinander differenziert herausgearbeitet werden müssen. 
In der oben erwähnten Studie zur Lernaufgabe pir@tes du net konnte beispielsweise die Überlagerung eines typischen individuellen Lernhandlungskonzepts, wie z.B. die Orientierung am freien Sprechen durch typische klasseninterne Rollenzuweisungen (z.B. Moderator, Klassenclown oder stiller Beobachter), nachgewiesen werden ebenso wie durch eine positive Bewertung sprechfördernder Lehrhaltungen. Auf Seiten der Unterrichtenden zeigten sich überlappende Orientierungen am Steuerungsmonopol und einer im Einzelfall ausgeprägten Neigung zur Selbstinszenierung.

Festzuhalten ist, dass die Mehrdimensionalität des jeweiligen Falles auf die Vielschichtigkeit der Erfahrungsräume zurückgeht. Für Schüler ist ihre Klasse ein Erfahrungsraum neben anderen: In der klasseninternen Interaktion können z.B. auch die Erfahrungsräume eines bestimmten familialen Hintergrundes, eines bestimmten Bildungshintergrundes, geschlechtsspezifische Erfahrungen und sprachlernspezifische Erfahrungen wie etwa Brieffreundschaften, Urlaubsreisen in das Zielland u. Ä. aufleuchten. Eine große Herausforderung für Forschende bilden die Ausprägungen der individuellen Sprachbewusstheit, die die klasseninterne Interaktion auf grundsätzlicher Ebene beeinflussen, transformieren, ja deformieren kann. Auf Lehrerseite wiederum können z.B. die eigene Schulbiografie, die Sprachlernbiografie, interkulturelle Erfahrungen, die familiale Sozialisation, die Studienerfahrungen an einer bestimmten Hochschule, die Ausbildungserfahrung bei einer bestimmten Seminarleiterin, die Generationenerfahrung (etwa zwischenzeitliche Akademikerarbeitslosigkeit und damit verbundener Ausschluss von Ressourcen) zusammenspielen und die Mehrdimensionalität eines konkreten Falles konstituieren. Die beobachteten Ausschnitte aus Klasseninteraktionen oder aus narrativen Biografien sind mithin immer Ausschnitte aus einer Gesamtformung, die es zu rekonstruieren gilt: Im Sinne Max Webers treten zu den Um-zu-Motiven die Weil-Motive (s. Bohnsack 2014: 146). So folgt beispielsweise das autoritäre Lehrerhandeln im Unterricht dem Motiv, ein bestimmtes als störend interpretiertes Verhalten gar nicht erst zuzulassen („um zu“), resultiert aber möglicherweise aus Erfahrungen in der eigenen Schulsozialisation („weil“). Die Aufdeckung der Weil-Motive steht für den Anspruch der Dokumentarischen Methode, eine soziogenetische Interpretation zu ermöglichen. Diese impliziert eine Typenbildung, die sowohl individual-psychologische als auch sozialpsychologische Erklärungen umfasst, d.h. Erklärungen, die auf das Individuum als schöpferische Einzelpersönlichkeit wie auch auf das Individuum als Teil einer sozialen Gruppe rekurrieren. Damit grenzt Bohnsack unter Rückbezug auf Mannheim die Dokumentarische Methode vom kausalgenetischen Erklärungsmuster Bourdieus (1976) ab, der den Habitus bzw. 
habituelles Verhalten generell als Ausdruck sozioökonomischer Verhältnisse interpretierte.

Die Typenbildung setzt die Interpretationsleistung des Forschers bzw. der Forschergruppe im Anschluss an die reflektierende Interpretation auf einer abstrakteren Ebene fort, gilt es doch, das „Typische“ eines Falles als Ganzes zu fassen. Dieser Prozess gleicht einem hermeneutischen Zirkel oder besser gesagt einer hermeneutischen Spirale, denn jeder weitere Typus, der an den Fall herangetragen wird, bedeutet eine Abstraktion, die die interpretatorische Ausgangslage wieder erweitert und verändert. Die Typenbildung wird auf diese Weise zu einem Prozess, der insofern eine gewisse Offenheit besitzt, da immer neue Erfahrungsschichten integriert werden müssen.

Je weniger Vergleichsfälle herangezogen werden können, umso eher ergibt sich bei den ermittelten Orientierungen das Problem geringer Kontrastierungen. Dies wiederum kann dazu führen, dass auch die so erschlossenen Typiken weniger komplex ausfallen oder dass eine Verortung des jeweiligen Falles in einer Typologie im Extremfall unmöglich ist. Dies ist meist auf der Ebene von kleineren Studien (z.B. Bachelor- und Masterarbeiten) der Fall, wie bei der Studie zum Comic Ne me quitte pas im Spanischunterricht einer 11. Klasse (s. Kap. 4.1). Es bedeutet allerdings keineswegs, dass die Ergebnisse für die fachdidaktische Theoriebildung weniger ergiebig sind, sondern es resultieren meist wertvolle Ergebnisse auf der Ebene der eruierten Rahmenorientierungen, die überraschende Einsichten in das Lehren und Lernen bieten können.

Zusammengefasst: In Kapitel 3.1 wurden die aus der rekonstruktiven Sozialforschung nach Bohnsack (2014) bekannten Arbeitsschritte auf den Untersuchungsgegenstand Fremdsprachenunterricht bezogen, mit Beispielen illustriert und reflektiert. Es ist festzuhalten, dass das Auftreten der fremdsprachigen Lernersprachen zu einer erheblichen Komplexitätserweiterung der Analysen führt. Auch die Fallbestimmung kann zu einer erhöhten Schwierigkeit werden, da es sich beim Fremdsprachenunterricht um sich überlagernde Bezugsfelder handelt, innerhalb derer eine Lerngruppe situiert ist. Diese Komplexität kann in der Fallbeschreibung reduziert und anschließend einer Typenbildung auf zwei Ebenen zugeführt werden, einer sinngenetischen - auf die Abgrenzung von Orientierungsmustern abzielende - und einer soziogenetischen Typenbildung. Letztere versucht, das Auftreten bestimmter Orientierungsmuster auf individual- und sozialpsychologische Ursachen zurückzuführen.

Im nun folgenden Kapitel werden einige Modifikationen der Gruppengesprächsanalyse nach Mangold und Bohnsack (1988) im Hinblick auf das narrative Interview vorgestellt. Diese Modifikationen bedingen zugleich kleinere Veränderungen bei den Arbeitsschritten. 


\subsection{Narratives Interview}

Das narrative Interview und die Gruppengesprächsanalyse sind insofern eng verwandte rekonstruktive Verfahren, als sie mit ähnlichen Mitteln - der Aufzeichnung und Interpretation sich selbstläufig entwickelnder Verständigung ähnliche Ziele verfolgen, insbesondere die Herausarbeitung von Typiken. Die Gemeinsamkeiten und Unterschiede wurden bereits in Kapitel 2.4 auf grundsätzlicher Ebene benannt. In diesem Kapitel werden sie auch auf der Ebene der Arbeitsschritte dargestellt. Da sich das narrative Interview und die Gruppengesprächsanalyse im Hinblick auf die Datenerhebung und -verarbeitung nur unwesentlich unterscheiden, genügt es, an dieser Stelle auf die Besonderheiten der Interpretation einzugehen. Dabei besteht ein Unterschied zwischen biografischen Interviews - nicht selten längeren zwei- bis dreistündigen Sitzungen und fokussierten kürzeren Interviews beispielsweise zur Frage der Lehrerfahrung bei Berufsanfängern oder zu Lernerfahrungen im Fremdsprachenunterricht der Mittelstufe. Aus der Praxis im Umgang mit dem fokussierten Kurzformat habe ich eine etwas modifizierte Vorgehensweise entwickelt, die im Folgenden zusammen mit und im Kontrast zu dem klassischen biografischen Interview vorgestellt wird.

\subsubsection{Formale Interviewanalyse}

Bei der Analyse von Gruppengesprächen wird nach der Transkription zunächst die thematische Gliederung des Textes vorgenommen, wohingegen am Anfang der Analyse eines narrativen biografischen Interviews die vorübergehende Ausklammerung nicht narrativer Passagen und die Sequenzierung der Erzählung stehen. Die Erzählung greift auf drei Grundformen der Darstellung zurück: die reine Erzählung, die Beschreibung und die Argumentation. Die reine Erzählung folgt der zeitlichen Entwicklung eines Ereignisses im Leben des Erzählers, während die Beschreibung und die Argumentation davon ablenken. Die reine Erzählung ist häufig detailreich im Hinblick auf zeitliche und räumliche Angaben sowie beteiligte Personen. Sie lenkt Forschende im Sinne der Homologieannahme (s. Kap. 2.4) auf den biografietheoretischen Erlebniskern. Gelingt es uns, diesen Erlebniskern in seiner Chronologie zu verfolgen bzw. herauszuarbeiten, so können wir ihn einer vertieften bzw. reflektierenden Interpretation unterziehen und zu Normerwartungen in Beziehung setzen (Abweichungen, Übereinstimmungen). Beschreibende Passagen enthalten häufig Zusammenfassungen oder Verallgemeinerungen, während Argumentationen meist der Rechtfertigung 
dienen. Sie werden erst zu einem späteren Zeitpunkt wieder zur Interpretation hinzugezogen.

Die vorherrschende Darstellungsform, die der Erzähler wählt, kann auf die aktuelle Bedeutung des Ereignisses hindeuten: Die Wahl einer Erzählung kann z.B. implizieren, dass der Erzähler eine Erfahrung abgeschlossen hat, während der Wechsel in eine andere Darstellungsform (Argumentation oder Beschreibung) darauf verweisen kann, dass Inhalte noch nicht komplett verarbeitet wurden oder problematisch sind (s. Lucius-Hoene \& Deppermann 2004: 171). Nicht immer ist eine eindeutige Identifikation der Darstellungsform möglich bzw. angebracht, da sie häufig ineinander übergehen oder stark verschachtelt sind. So können argumentative und beschreibende Aspekte eine hilfreiche Untermauerung für eine erzählerische Passage sein, denn die deskriptiven Teile dienen der „Entfaltung des Erzählraums“ (ibd.: 172), während die argumentativen Aspekte „Handlungspositionen und Motive, Ereignisse und Resultate plausibilisieren, rechtfertigen oder begründen" (ibd.). Gleichzeitig können beschreibende Teile durch erzählerische Schilderungen und Argumente unterstützt werden. Auch überwiegend argumentative Teile können von Erzählungen und Beschreibungen begleitet werden (s. ibd. 172 f.). Auch wenn laut Glinka (2008: 11) im narrativen Interview die Darstellungsform des Erzählens überwiegt, lassen sich gleichwohl häufig eingelagerte beschreibende sowie argumentierende Passagen wiederfinden.

Die Beschreibungen und Argumentationen können im Transkript farblich markiert oder durchgestrichen und ihr Ausschluss anschließend auf einem $\mathrm{Zu}$ satzdokument begründet werden. Die Sequenzierung erfolgt ebenfalls im Transkript selbst durch Hervorhebung von zeitlichen Markierern: Verben, Adverbien oder adverbialen Bestimmungen, sog. „Rahmenschaltelementen“ (Schütze 1984: 286; Kleemann 2009: 67), die den Bezug der Ereignisse markieren, z.B. das Vorangehen, die Parallelität oder das Nachfolgen von Ereignissen. Die Sequenzierung der Erzählung wird mit Zeilenangabe markiert.

Beim fokussierten Kurzformat des narrativen Interviews dagegen kann es sein, dass eine Sequenzierung nach den drei Darstellungsformen zunächst nicht sinnvoll ist, wenn nämlich die reine Erzählung nicht ergiebig genug ist (s. Kap. 4.2, „Bianca“ und „Anne“). Die Sequenzierung kann in diesem Fall auch nach der reflektierenden Interpretation vorgenommen werden, um die Funktion der nicht narrativen Darstellungsformen in der Gesamtgestalt des Interviews zu erklären. Der Fall „Bianca“ beispielsweise ist dadurch gekennzeichnet, dass Bianca fast nichts direkt über sich selbst erzählt, sondern überwiegend beschreibt und argumentiert und zwar vor dem Hintergrund, dass sie die Interviewerinnen, die 
noch Studentinnen sind, fast noch als ihre Peers betrachtet, ihnen jedoch zeigen will, dass sie einen kleinen, aber entscheidenden Vorsprung vor ihnen besitzt, nämlich Unterrichtspraxis. Das Motiv, den Erfahrungsvorsprung als Überlegenheit gegenüber den Interviewerinnen auszuspielen, hebelt in gewissem Sinne das Interview als Interview aus und verwandelt es in eine Bühne für die Peer-Interaktion. Ungewollt werden die beiden Interviewerinnen zu Gesprächspartnerinnen in einer Art Gruppendiskussion, in der aber nur eine Person spricht. Dennoch ist dieses Interview als Datengrundlage wertvoll, da es Aufschluss über die Orientierungen junger Fremdsprachenlehrerinnen in der Berufseinstiegsphase gibt.

\subsubsection{Inhaltliche Interviewanalyse}

Wie bei der Analyse von Gruppengesprächen beginnt die Analyse beim narrativen Interview mit einer resümierenden Kurzzusammenfassung bzw. Paraphrasierung der Einzelsequenzen. Das heißt, man schreitet von Markierer zu Markierer voran und reformuliert den Inhalt in eigenen Worten. Gleichzeitig erhält jede Sinneinheit, die unter Umständen mehrere Sequenzen umfasst, eine Kurzüberschrift.

Erst danach erfolgt die vertiefte reflektierende Interpretation ${ }^{25}$ der zuvor identifizierten Sinneinheiten. Nunmehr werden die Ereignisse vor der Folie der vier idealtypischen Prozessstrukturen des Lebenslaufs (s. Schütze 1983, s. Kapitel 2.4) kategorisiert und mit dem Ziel reflektierend interpretiert, die Gesamtstruktur (biografische Gesamtgestalt, s. Kap. 2.4) des Falles über die Identifizierung und Analyse seiner „Zugzwänge“ sowie seiner Sequenzialität herauszuarbeiten. Vergleichshorizont ist dabei aber nicht nur die Folie der idealtypischen Prozessstrukturen, sondern auch das fiktive ,Andere', das zwar ,Normalitätsvorstellungen' entspricht, diese aber individuell ausgelegt. Normerwartungen werden also innerhalb der Dokumentarischen Methode - anders als bei der objektiven Hermeneutik nach Oevermann (1979) - nicht gedankenexperimentell konstruiert, sondern auf empirischer Basis durch einen Prozess des Vergleichens ermittelt.

25 Der Begriff reflektierende Interpretation taucht in der Terminologie Schützes nicht auf, sondern stammt aus den Arbeiten Bohnsacks. Ich verwende ihn jedoch auch bei den eigenen Forschungen zum narrativen Interview ebenso wie den Begriff der formulierenden Interpretation, da ich in Übereinstimmung mit Nohl (2012) keinerlei Widerspruch in dieser begrifflichen Übertragung sehe. Ganz im Gegenteil, auch beim narrativen Interview wird der Schritt des Wechsels der Analysehaltung vom Was zum Wie vollzogen, um die tieferen Schichten der Verständigung herauszuarbeiten. Die Benutzung ein und derselben Begrifflichkeit betont demnach die methodischen und methodologischen Gemeinsamkeiten. 
Dazu werden nun auch die zuvor ausgeklammerten Beschreibungen und Argumentationen herangezogen. Die Gesamtstruktur erklärt das Übereinstimmen oder Abweichen biografischer Ereignisse von Normerwartungen. Dieses Übereinstimmen oder Abweichen kann punktuell erfolgen oder längere Zeiträume, eventuell auch das ganze Leben umfassen. Im Vergleich mit anderen Fällen kann sich aber auch herausstellen, dass ehemalige Normerwartungen mit der Zeit obsolet geworden sind und durch andere, neue Normerwartungen überlagert werden.

Beim fokussierten Kurzinterview ist auch ein paralleles Umsetzen der Arbeitsschritte möglich: Die Grobthemen werden chronologisch in einer Spalte angeordnet, die thematische Feingliederung in der zweiten Spalte, die formulierende Interpretation in der dritten Spalte, die reflektierende Interpretation in der vierten Spalte und die Normalitätsvorstellungen in der fünften Spalte. Die fünfte Spalte "Normalitätsvorstellungen“ wird im Wechselspiel mit der vierten Spalte „reflektierende Interpretation“ parallel gefüllt, da sie sozusagen die Rolle des Vergleichshorizonts in der Diskursanalyse einnimmt, wie die Ausschnitte aus den Fällen „Bianca“ und „Anne“ (s. Kap. 4.2) zeigen.

Tabelle 7: Interpretationsraster für biografische Kurzinterviews

\begin{tabular}{|c|c|c|c|c|}
\hline Sequenz & $\begin{array}{l}\text { Thematische } \\
\text { Gliederung }\end{array}$ & $\begin{array}{l}\text { Formulierende } \\
\text { Interpretation }\end{array}$ & $\begin{array}{l}\text { Reflektierende } \\
\text { Interpretation }\end{array}$ & $\begin{array}{l}\text { Normalitäts- } \\
\text { vorstellungen }\end{array}$ \\
\hline $\begin{array}{l}\text { I } \\
\text { Z. 05-23 } \\
\text { Berufswahl }\end{array}$ & & & & \\
\hline II & & & & \\
\hline Etc. & & & & \\
\hline
\end{tabular}

\subsubsection{Fallbeschreibung und Fallvergleich}

Steht am Ende von Arbeitsschritt 3.2.2 eine Beschreibung der einzelnen Fälle, so wird diese Fallbeschreibung im letzten Arbeitsschritt über den Vergleich mehrerer Fälle wie bei der Gruppengesprächsanalyse zu einer Typik weiterentwickelt. Bereits im Arbeitsschritt der inhaltlichen Analyse findet ein fallinterner Vergleich statt: Es werden Episoden innerhalb der chronologischen Gesamtentwicklung des Falles verglichen. Dabei können bereits persönlichkeitstypische Einstellungen bzw. Orientierungen eruiert werden. Nunmehr geht es darum, gleich oder ähnlich gelagerte Fälle miteinander zu vergleichen, um z. B. generationentypische, 
geschlechtertypische, bildungsgang- oder bildungsstatutstypische Orientierungen herauszufinden. Beispiele dafür bietet Kapitel 4.2.

Zusammenfassend lässt sich festhalten, dass es ohne Weiteres möglich ist, die Dokumentarische Methode auf die Interviewanalyse zu übertragen. Dies ist in der rekonstruktiven Fremdsprachenforschung sogar ausgesprochen wünschenswert, da dieses Vorgehen eine Triangulation unterrichtsbasierter Daten und retrospektiver Interview- oder Gruppengesprächsdaten erlaubt. Die Rekonstruktion von Verständigung im Unterricht kann auf diese Weise mit ihrer Rekonstruktion im Interview oder Gruppengespäch verglichen werden.

Im nun folgenden fünften Kapitel stelle ich eine kleine Auswahl von Fallanalysen vor, die einen Einblick in das Potential der rekonstruktiven Fremdsprachenforschung mit der Dokumentarischen Methode bieten. Das begriffliche Element „Werkstatt“ in der Kapitelüberschrift weist darauf hin, dass es sich um Abschnitte im Forschungsprozess, um Interpretationsschritte und um vorläufige Lesarten handelt, die die Funktion haben, konkrete Einblicke in den Forschungsprozess zu geben. 


\section{Fallwerkstatt}

Mit diesem Kapitel betreten wir die Forschungswerkstatt der rekonstruktiven Fremdsprachenforschung und verfolgen einige Arbeitsschritte der Methode zu konkretem Datenmaterial.

Die Unterrichtstranskripte zur Bearbeitung des Comics Ne me quitte pas (Jordi Lafebre 2010), die Aufgabenstellungen und die Fallbeschreibungen sind einer Staatsexamensarbeit entnommen (Winkels 2015). Einige dieser Materialien wurden, wie auch die narrativen Interviews mit Lehrenden, im Rahmen von Forschungsseminaren (Wintersemester 2014-2015 bis Wintersemester 2015-2016) an der Universität Kassel diskutiert. Alles in allem könnte dieses mit Studierenden generierte Material dazu beitragen, die Hemmschwelle bei der Wahl der Dokumentarischen Methode auch mit Blick auf den wissenschaftlichen Nachwuchs zu senken.

Kapitel 4.1 führt zunächst in das Konzept der Fallwerkstatt ein. In Kapitel 4.1.2. wird anschließend der Textsinn des Comics Ne me quitte pas und in Kapitel 4.1.3 der Aufgabensinn der zu diesem Comic von Winkels (2015) generierten Lernaufgabe rekonstruiert. Die Kapitel 4.1.4, 4.1.5, 4.1.6 erlauben es den Lesern, die Praxis der rekonstruktiven Erforschung der Aufgabenbearbeitung im beobachteten Unterricht nachzuvollziehen. Es werden konkrete Ergebnisse einzelner Arbeitsschritte präsentiert und im Forschungsseminar aufgetauchte Schwierigkeiten sowie diesbezügliche Überlegungen und Entscheidungen transparent gemacht. In Kapitel 4.2 werden die Arbeitsschritte der Analyse zweier narrativer Interviews mit Junglehrerinnen, Bianca und Anne, präsentiert, die über ein Jahr Berufserfahrung nach dem Referendariat verfügen und von ihren ersten Erfahrungen als Spanisch- und Französischlehrerin erzählen.

\subsection{Der Comic Ne me quitte pas im Spanischunterricht}

\subsubsection{Forschungsfragen und Forschungsrahmen}

Die Verständigung über Texte kann als Kernkomponente des Fremdsprachenunterrichts bezeichnet werden. Sie erfolgt im Fremdsprachenunterricht in aller Regel aufgabengeleitet: Texte im Sinne des erweiterten Textbegriffs (kontinuierliche oder diskontinuierliche Texte; Schrift-, Bild, Ton- oder Filmtexte) werden innerhalb eines bestimmten Aufgabenarrangements rezipiert, das heißt verarbeitet bzw. verstanden, und meist werden dabei eigene Texte produziert. Aus praxeologischer Sicht stellen sich somit Forschungsfragen hinsichtlich der Sinnkonstruktionen im fremdsprachlichen Klassenzimmer: Welche impliziten bzw. atheoretischen 
Wissensbestände (Weltwissen, Textwissen, lernersprachliches, lernstrategisches, interaktionales Wissen) werden bei der Sinnkonstruktion aktiviert? Welche Orientierungen bezogen auf die Textbearbeitung (im Sinne von Rezeption und Produktion) dominieren in einer bestimmten gegebenen Gruppe in einer bestimmten Arbeitsphase? Welche Rückschlüsse lassen sich daraus im Hinblick auf Angebot und Nutzung einer Lernumgebung (s. Kap. 1) ziehen? Welche Schlüsse resultieren daraus für eine eventuelle Modifizierung der Aufgaben? Welche Schlüsse resultieren daraus im Hinblick auf die Individualisierung des Unterrichts? Die Komplexität und Diversität der Lernprozesse wird sich auch hier gemäß der theoretischen Annahmen zur Sozialität des Lernens (s. Kap. 2.1) in einer Fülle geschachtelter Typiken offenbaren, die es empirisch herauszuarbeiten gilt.

Im Folgenden werden Daten zur Analyse eines Comics im Spanischunterricht einer elften Klasse vorgestellt, die in einer Staatsexamensarbeit (Winkels 2015) an der Universität Kassel im Sommersemester 2015 sowie in einem fachdidaktischen Forschungsseminar im Wintersemester 2015-2016 bearbeitet wurden. Es handelt sich um den 2010 erschienen Text Ne me quitte pas des katalanischen Comicautors Jordi Lafebre, erschienen im Sammelband Barcelona TM.

In den Vorgesprächen zu ihrer Staatsexamensarbeit äußerte die Studierende den Wunsch, die Bearbeitung eines literarischen Textes im Spanischunterricht zu untersuchen. Zu diesem Zweck entwickelte sie für fünf Unterrichtsstunden vier Arbeitsblätter mit sechs Hauptaufgaben, die zum Teil jeweils noch einmal in mehrere Unteraufgaben untergliedert waren. Dieses Unterrichtsvorhaben kann zwar auf Grund des durch die Studie bedingten Wechsels der Lehrperson - die Studierende führte den Unterricht selbst durch - aber auch möglicherweise auf Grund der Themenwahl und der spezifischen Aufgabenkonzeption nicht als typisch für den Unterricht in der Klasse bezeichnet werden. Es erlaubt gleichwohl eine Untersuchung von Orientierungen im Rahmen eines Einzelfalles (zur Definition eines Falles s.o. Kap. 3.1.4). Das Forschungsinteresse bestand im Rahmen der Hausarbeit darin, die aufgabengeleitete Verständigung über einen bimodalen literarischen Kurztext - als literarische Anschlusskommunikation bezeichnet mit Hilfe eines rekonstruktiven Verfahrens zu untersuchen.

Es war klar, dass sich im Rahmen dieser Arbeit nicht mehr als eine Klasse für die Untersuchung heranziehen ließe. Die Klassenaquise, die Transkriptionsarbeit und die Interpretationen mussten der Ökonomie der Einzelforschung im Umfang einer Staatsexamensarbeit untergeordnet werden. Auf mein Anraten hin nahm die Studierende am regelmäßig angebotenen Forschungsseminar teil, wo sie die Grundlagen der rekonstruktiven Fremdsprachenforschung und die Arbeitsschritte der Dokumentarischen Methode kennenlernte. Die Studierende 
arbeitete für die Interpretationen überdies mit einer Kommilitonin zusammen, die ebenfalls am Forschungsseminar teilgenommen hatte, mit dem Ziel, auf diese Weise ein Minimum an kommunikativer Validierung zu erreichen.

Im Betreuungsgespräch wurden auch Fragen der Reichweite und Validität der Ergebnisse angesprochen: Auf welchem Forschungsinteresse beruht eine Studie in einer einzigen Klasse mit nur elf Schülerinnen und Schülern? Lassen sich auf dieser Basis überhaupt Typiken generieren? Es wurde vereinbart, die Klasse in Gruppen à drei bis vier Schüler zu teilen, so dass lerngruppenbasierte Fälle miteinander verglichen werden konnten. $\mathrm{Zu}$ erwarten waren individuelle Bedeutungsaushandlungen, die fallintern und fallübergreifend eine zwar schwache, aber dennoch vorhandene Kontrastbildung erlaubten und Anhaltspunkte für literarisches fremdsprachliches Verstehen im Sinne des Fremdverstehens nach Bredella (2007, s. Kap. 2.2 und 2.3) bieten würden. Die Erwartung war ferner, angesichts der Deutungsoffenheit literarischer Texte Einzelfalltypiken zu ermitteln.

Im Rahmen des im Folgesemester durchgeführten Forschungsseminars mit Lehramtsstudierenden konnten einige der Audioaufnahmen dann noch einmal nachgenutzt werden. Ziel dieses Seminars war es, in die rekonstruktive Fremdsprachenforschung einzuführen, um Studierenden Einblicke in fremdsprachenunterrichtliche Interaktionen zu ermöglichen, den Kandidatinnen und Kandidaten für eine Staatsexamensarbeit forschungsmethodische Anregungen zu geben sowie Sensibilität für Forschungsfragen zu wecken. Auf diese beiden Quellen, der Staatsexamensarbeit (Winkels 2015) und die Diskussionen im Forschungsseminar, gehen einige der folgenden Ausführungen zurück. Sie werden als „Fallwerkstatt“ bezeichnet, da sie methodische Entscheidungen sowie Deutungsalternativen nachvollziehbar machen, die für die Arbeit mit der Dokumentarischen Methode in der fremdsprachenunterrichtlichen Forschungspraxis charakteristisch sind.

\subsubsection{Jordi Lafebre, Ne me quitte pas: Analyse des Textsinns}

Der Kurz-Comic Ne me quitte pas besteht aus sieben Seiten inclusive Deckblatt und insgesamt 36 Einzelbildern, alles in Schwarz und Weiß. Er gibt das Zwiegespräch eines jungen männlichen Einwohners von Barcelona mit seiner Heimatstadt wieder, d.h. die Stadt wird personifiziert und erhält eine eigene Stimme. Thema des Zwiegesprächs ist die besondere Beziehung - eine Art Liebesbeziehung - dieser Person zu ihrer Stadt. Der Mann denkt zwar darüber nach, seine Heimatstadt zu verlassen, entscheidet sich aber am Ende für das Bleiben. Im Laufe der 36 Bilder werden verschiedene Argumente für das Wegziehen benannt. Ihnen werden jeweils Gegenargumente, der Stadt' gegenübergestellt. 
Die bimodale künstlerische Gestaltung macht die Textsorte Comic zu einem didaktisch sehr interessanten Lerngegenstand: Bild und Text verweisen aufeinander, deuten sich wechselseitig. Jedes Bild und jeder Textabschnitt besitzen eine eigene Bedeutungsstruktur. Zudem gibt es ständige Verweise von einem Bild auf das nächste, von Bild zu Text und umgekehrt, so dass sich neben der Indexikalität bezogen auf externe Wissensbestände eine reichhaltige interne Verweisstruktur herausarbeiten lässt. Im Folgenden wird der Textsinn des Titelbilds exemplarisch analysiert.

Das Titelbild weist eine planimetrische Komposition auf, die eine gezeichnete Person in einem Rahmen vor einem geteilten Hintergund zeigt. Der Leser weiß zu diesem Zeitpunkt nicht, dass dieses eingefügte Bild der Person auf Seite drei des Comics wieder auftaucht. Die obere Hälfte des Hintergrunds zeigt eine Mauer, die untere Hälfte ist weiß. Die Gestaltung des Titelphotos generiert eine Mehrdeutigkeit: Es gibt rundherum Druckerschwärze, daher könnte es sich auch um einen Stempel handeln oder eine Art Abziehbild. Die Zentralperspektive des gezeichneten Photos lenkt den Blick des Lesers, es gibt nur das Photo, die Mauer und die Schriftzüge.

Abbildung 8: Titelbild des Comics Ne me quitte pas (Lafebre 2010, Copyright Norma Editorial, Barcelona)

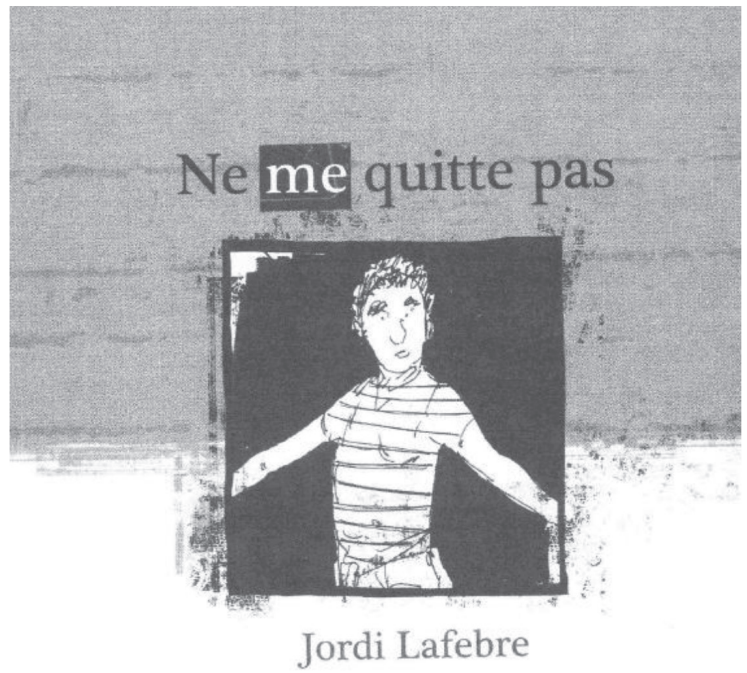


Der Blick des Lesers wird sodann auf den Titel gelenkt - er befindet sich oberhalb des gezeichneten Photos - und auf den Autorennamen, der sich direkt unterhalb des Photos befindet. Im französischsprachigen Titel Ne me quitte pas ist das Pronomen me durch den umgekehrten Schrift-Hintergrundkontrast hervorgehoben. Zum kommunikativen Wissen der Leserschaft gehört, dass Ne me quitte pas, der gleichnamige Titel eines berühmten Chansons von Jacques Brel, auf Deutsch „Verlass mich nicht“ bedeutet.

Der Name des Zeichners Jordi Lafebre ist so unter das Photo gesetzt, dass er suggeriert, vielleicht selbst der Mann auf dem gezeichneten Photo zu sein. Somit fügen sich Mauer, Photo und Schriftzüge zu einer Art Choreografie symbolischer Bezüge, die erste Assoziationen wecken. Handelt es sich um eine Gefangenschaft? Ist das Photo ein Suchphoto? Gibt es eine tragische Liebesgeschichte wie im Chanson von Jacques Brel?

Diese detaillierte dokumentarische Analyse des Titelblatts könnte als Blaupause für die Analyse weiterer Einzelbilder dienen, auch im Unterricht selbst, wird aber aus Platzgründen hier nicht weiterverfolgt.

Die erste ,erzählende' Seite des Comics beginnt mit der Aussage Estoy un poco harto de tí, la verdad (Du gehst mir wirklich etwas auf die Nerven, Übers. BT). Der Inhalt dieses Satzes wird forthin ausgeführt, d.h. der Leser erfährt, was genau der Person an seiner Stadt auf die Nerven geht. Die Stadt antwortet jeweils mit einer Widerrede oder einer Rechtfertigung. Die Argument-Struktur ist folgendermaßen aufgebaut, d.h. der kommunikative Sinn des Schrifttexts lässt sich folgendermaßen rekonstruieren:

Tabelle 8: Argumentstruktur des Comics Ne me quitte pas

\begin{tabular}{|l|l|l|}
\hline Seite & Person & Stadt \\
\hline 1 & $\begin{array}{l}\text { Ist von seiner Stadt genervt. } \\
\text { Äußert, dass er sich ausgenutzt fühlt. }\end{array}$ & $\begin{array}{l}\text { Hält das gemeinsame Leben dagegen. } \\
\text { Hält die gemeinsamen Anstrengungen } \\
\text { dagegen und dass es beiden jetzt } \\
\text { gutgehe. }\end{array}$ \\
& $\begin{array}{l}\text { Behauptet, dass nur die Stadt vom } \\
\text { Aufschwung profitiere und wirft } \\
\text { Ehr vor, nicht mehr die Sprache der } \\
\text { Einwohner (catalán) zu sprechen. } \\
\text { sei und dass die Vermeidung des } \\
\text { Katalanischen plausible Gründe habe, } \\
\text { nämlich Tourismus und Zuzug. }\end{array}$ \\
& $\begin{array}{l}\text { Räumt ein, dass es für die Olympischen } \\
\text { Spiele vielleicht noch gerechtfertigt war. }\end{array}$ & $\begin{array}{l}\text { Insistiert, dass sie im Kern immer } \\
\text { noch die Alte sei, sich lediglich } \\
\text { an den äußeren Wandel und die } \\
\text { Globalisierung angepasst habe. }\end{array}$ \\
\hline
\end{tabular}




\begin{tabular}{|c|c|c|}
\hline Seite & Person & Stadt \\
\hline & $\begin{array}{l}\text { Wirft ihr absichtliche Vagheiten und } \\
\text { unerfüllte Versprechen vor. }\end{array}$ & $\begin{array}{l}\text { Evoziert die schönen Bilder der } \\
\text { Vergangenheit (Kindheit, Partys, den } \\
\text { ersten Kuss, Fahrradfahren in der } \\
\text { Stadt). }\end{array}$ \\
\hline \multirow[t]{2}{*}{3} & $\begin{array}{l}\text { Hält dagegen, dass sie sich von ihm } \\
\text { entfernt habe, zu den Schönen und } \\
\text { Reichen gehören wolle. }\end{array}$ & $\begin{array}{l}\text { Widerspricht und behauptet, nur zu } \\
\text { ihm eine echte Beziehung zu haben. }\end{array}$ \\
\hline & $\begin{array}{l}\text { Wirft ihr vor, nur deshalb um ihn zu } \\
\text { werben, weil auch andere sich für ihn } \\
\text { interessierten. }\end{array}$ & $\begin{array}{l}\text { Reagiert mit gespieltem Stolz und } \\
\text { brüstet sich ihrer Bedeutung für ihn. }\end{array}$ \\
\hline 4 & $\begin{array}{l}\text { Wirft ihr Heuchelei vor und dass sie } \\
\text { sich nicht mehr für die eigenen Leute } \\
\text { interessiere. }\end{array}$ & $\begin{array}{l}\text { Deutet ihr Verhalten als Lebenskunst } \\
\text { und sein Verhalten als altmodisch } \\
\text { und rückständig und seinerseits } \\
\text { heuchlerisch, da er sich darin gefalle, } \\
\text { seine Herkunft zu benennen. }\end{array}$ \\
\hline \multirow[t]{2}{*}{5} & $\begin{array}{l}\text { Rechtfertigt sich damit, den Menschen } \\
\text { lediglich den wahren Kern der Stadt } \\
\text { zu erklären, ihre Verborgenheit und } \\
\text { Schönheit. }\end{array}$ & Wirft ihm vor, stehengeblieben zu sein. \\
\hline & Behauptet, sich selbst treu zu sein. & $\begin{array}{l}\text { Wirft ihm Heuchelei vor und bietet } \\
\text { ihm an, sich doch von ihr zu trennen. }\end{array}$ \\
\hline 6 & $\begin{array}{l}\text { Gesteht, dass er es nicht fertig bringe, } \\
\text { da er sie liebe. }\end{array}$ & \\
\hline
\end{tabular}

Für die Entschlüsselung der dokumentarischen Textaussage ist es im nächsten Arbeitsschritt erforderlich, die Bildaussage heranzuziehen. Sie liefert para-verbale piktorale Hinweise auf weitere Sinnebenen. So werden beispielsweise die Abgegrenztheit des individuellen Erfahrungsraums und die Komplexität des urbanen Erfahrungsraums zeichnerisch kontrastiert. Die Bilder, die sich auf die Einzelperson beziehen, zeigen nur diese allein, einen bestimmten Gemütszustand (genervt, verliebt, verträumt etc.), meist ohne Hintergrund, wohingegen die Bilder, die sich auf die Perspektive der Stadt beziehen, meist komplexe Arrangements wiedergeben (Gebäude, Straßen, Verkehr, etc.), mit einem detailliert ausgestalteten Hintergrund. Das Individuum wird als abgegrenztes, etwas kauziges aber liebenswertes Original gezeichnet. Die Bartstoppeln, das ungekämmte Haar, die rudimentäre Kleidung des ,Ureinwohners' kontrastieren mit der für Touristen herausgeputzten Fassade der Stadt. Die Stadt erscheint in zwei Bildern symbolhaft personifiziert. Auf Seite vier erscheint sie als schwarzes Monster, das den Mann in die Wange kneift, und auf Seite fünf im Doppelbild einer alten Frau, 
einmal von der Seite gesehen, das Gesicht somit abgewandt bzw. unzugänglich, und einmal als schwarze Silhouette.

Am Ende versöhnen sich beide in dem Schlussmotiv des Gründungsmärchens, dessen Anfangsformel im letzten Bild über der Fassadensilhouette der Stadt und einer sonderbaren, schratartigen Zaubererfigur erscheint, die den Mann als gealterten Märchenerzähler zeigt.

In diesem Comic wird die Frage nach dem Leben, ja Überleben des Individuums im urbanen Großraum aufgeworfen, damit verbunden das Thema der verschiedenen Erfahrungsräume in der Großstadt, und es wird die Vision einer Liebesbeziehung zwischen Großstadt und Großstadtmensch entworfen.

\subsubsection{Ne me quitte pas: Analyse des Aufgabensinns}

Im Folgenden wird die Aufgabe als task as a workplan (Ellis 2003), d.h. als Arbeitsauftrag analysiert. Das Ziel ist, den intendierten bzw. potentiellen Aufgabensinn herauszuarbeiten (s. Kap. 2.3). Lerngruppenmerkmale, Sprachenfolge der Lerngruppe, Lernstand, Textpotential und Zielkompetenzen spielen dabei zusammen und können neben dem Aufgabenarrangement als die grundlegenden Komponenten von Lernaufgaben betrachtet werden.

Winkels (2015) entwickelte im Vorfeld ihrer Unterrichtsbeobachtung zur literarischen Anschlusskommunikation in einer Lerngruppe der elften Jahrgangstufe fünf Arbeitsblätter für eine Unterrichtssequenz von zwei Doppelstunden und einer Einzelstunde. Die Klasse eines Oberstufengymnasiums befand sich im dritten Lernjahr Spanisch und lernte diese Sprache als dritte Fremdsprache nach Englisch und Französisch.

Die beobachtete Lerngruppe setzt sich aus sieben Schülerinnen und vier Schülern im Alter von 17 bis 18 Jahren zusammen. Ihre Motivation und Leistungsbereitschaft werden von Winkels als durchschnittlich eingeschätzt, vier Gruppenmitglieder ragen nach oben heraus, drei eher nach unten. Dies korrespondiert mit den Leistungen, die von Winkels gemäß den Stufen des GeR auf Niveau A2 bis A2+ eingeschätzt werden. Allerdings ist dabei nicht das mehrsprachige didaktische Vorwissen berücksichtigt, d.h. das Wissen, das bei dieser Lerngruppe aus den Lernerfahrungen mit den vorgelernten Sprachen Englisch und Französisch resultiert. Hinzu kommt, dass das verwendete Lehrwerk Adelante intermedio (Klett 2011) auf das Erreichen von Niveau B1 abzielt. Es wäre also denkbar, dass einige Schüler dieses Niveau zum Zeitpunkt der Unterrichtsbeobachtung bereits in Teilen (z.B. Textverstehen) erreicht bzw. sogar überschritten haben. Die Klasse ist nach Auskunft ihrer Lehrerin an vielfältige Methoden und die weitgehende Verwendung des Spanischen als Arbeitssprache im Unterricht 
gewöhnt. Sie steht zum Zeitpunkt der Unterrichtsbeobachtung bei Lektion drei Cataluña, d.h. der Text von Jordi Lafebre schließt thematisch an den aktuell behandelten thematischen Lerngegenstand Katalonien an.

Um das Textpotential von Ne me quitte pas im Hinblick auf die beschriebene Lerngruppe einzuschätzen, ist an das Medium des Comic an sich sowie an die spezifische Textkomplexität zu erinnern. Comics eignen sich häufig schon auf Grund der Bild-Schrift-Bimodalität für eine Verwendung im Fremdsprachenunterricht, denn die Bildaussage kann die Schriftaussage stützen und umgekehrt. Dies ist auch bei diesem Comic streckenweise der Fall: Die Stimmungen des Bewohners von Barcelona werden durch den starken Gesichtsausdruck vermittelt, juegos en la calle (Spielen auf der Straße, alle Übersetzungen forthin BT) durch das Jungengesicht, las noches de fiesta (durchgefeierte Nächte) durch die Tanzenden in der Nacht, el primer beso (der erste Kuss) und paseos en bici (Fahrradfahren) durch entsprechende eindeutige Bilder. Andere Bilder unterstützen die Schrifttextaussage nur sehr indirekt (symbolisch oder expressionistisch): Ein Verkehrsstau und Mülltonnen stehen für tantos esfuerzos (die vielen Anstrengungen), aber auch für Alltag, der obeliskartige Lápiz (ein charakteristisches Gebäude, das einem riesigen Bleistift ähnelt) für den Tourismus, Straßen und Häuserzeilen für dauerhafte Erinnerung, ein entstelltes Touristengesicht für Gesichtslosigkeit, Baukräne für Weiterentwicklung, alte Frauen für cuidabas tus cosas de siempre, intima y hechizante (du hütetest deine alten Sachen, warst verschlossen und bezaubernd), eine orientalische Schratgestalt für einen alten Märchen- und Legendenerzähler. Diese Assoziationen wiederum können die Phantasie anregen, aber auch das Verständnis erschweren und konstituieren neben anderen Merkmalen die Bild-Text-Komplexität. Die sprachliche Komplexität des Comic entspricht in etwa dem Verstehensniveau B1 und wird durch die Vorentlastung (s. u. Arbeitsblatt I) und die zusätzlichen Vokabelhilfen auch für untere Niveaus zugänglich.

Mit Bezug auf die kulturell-inhaltliche und ästhetische Komplexität des Textes und seinen motivationalen Gehalt für die Verwendung im Spanischunterricht einer elften Klasse lässt sich folgendes anmerken. Die Großstadt Barcelona als Unterrichtsthema ist ein für Jugendliche meist motivierender Lernanlass und das Thema der Heimatverbundenheit gut zugänglich. Es bietet in der Regel Anknüpfungs- und Identifikationspunkte. Weniger zugänglich sind dagegen möglicherweise die konkreten Detailbezüge, d.h. die abgebildeten Stadtausschnitte. Sie sprechen eher Personen an, die Barcelona bereits sehr gut kennen und ihr eigenes Barcelona-Bild mit dem des Zeichners abgleichen können. Dennoch ist es natürlich möglich, im Spanischunterricht die Auswahl der Motive zu reflektieren und daraus das spezifische Barcelona-Bild des Autors zu rekonstruieren. 
Verkehrschaos im Regen, generell Regen- und Winteransichten mit Bäumen ohne Blätter, Ampeln, Mülltonnen, Fahrradfahren sind Stadteindrücke, die den ganzjährigen Alltag und das Leben inklusive seiner Schattenseiten betonen. Touristen kommen hingegen in erster Linie in den besseren Jahreszeiten, und sie nehmen die Postkartenmotive der Stadt mit. Dieses Postkartenbild wiederum ist das Werbebild der Stadt. Es dient vor allem dazu, die Stadt zu vermarkten. Der Aufgabensinn umfasst auch das Verstehen dieser kulturell-inhaltlichen und ästhetischen Komplexität des Textes.

Die Zeichentechnik ordnet sich den genannten Kontrastierungen unter. Die Schwarz-Weiß-Bilder, die Strichtechnik, Verzerrungen und Verfremdungen können als Diskursmerkmale rekonstruiert werden. Sie konterkarieren das Postkarten- und Hochglanzimage der Stadt, das die Lernenden aus ihren Lehrbüchern gewohnt sind und provozieren möglicherweise eine ästhetische Irritation. Das Potential des Comic liegt daher auch in dieser möglichen Irritation. Sie aufzudecken und zu reflektieren, ihr die Alltagserfahrung der dargestellten Person zuzuordnen und gegebenenfalls mit den Alltagserfahrungen in der eigenen Stadt bzw. Lebenswelt zu vergleichen, könnte zu einer identifikatorischen Leseerfahrung im Sinne der von Nünning (2007: 126) genannten vierten Dimension des Verstehens literarischer Texte führen:

Fremdverstehen im Sinne des Einfühlens in bzw. Verstehens von fiktionalen Figuren aus einer fremden Kultur, also eine komplexe Mischung von interpersonalen, literarischen und fremdkulturellen Verstehensprozessen.

(Nünning 2007: 126; s.o. Kap. 2.2)

Im Folgenden werden die Arbeitsaufträge beschrieben, die zusammen - allerdings mit großen Einschränkungen - als workplan einer komplexen literarischen Lernaufgabe für untere GeR-Niveaus betrachtet werden können und den Aufgabensinn beinhalten. Sie erfüllen grosso modo die allgemeine Definition nach Caspari \& Burwitz-Melzer (2016: 248), die unter einer Lernaufgabe Folgendes verstehen:

[...] ein thematisch gerahmtes Arrangement aus Einzelaufgaben (Aufgabe 1, 2, 3 etc.), die jeweils auf eine bestimmte Kompetenz fokussieren. Andere Kompetenzen werden in diesen Einzelaufgaben zwar berührt bzw. sind integrativ, stehen jedoch nicht im Mittelpunkt. Einzelaufgaben können aus mehreren Teilaufgaben (1a, 1b etc.) bestehen. Häufig bereiten die Einzelaufgaben inhaltlich, sprachlich und methodisch auf eine Zielaufgabe (target task) vor, in der ein bestimmtes Produkt erstellt werden muss.

Von den weiteren genannten Merkmalen „Authentizität bzw. Relevanz“, „Auslösen von sprachlichen Lernprozessen“, „Produktorientierung“ und „Inhaltsorientierung“ (ibd.) treffen in der geplanten Aufgabe und in Anlehnung an 
Thonhauser (2010) zwar das „Auslösen von sprachlichen Lernprozessen“ sowie „Inhaltsorientierung“ zu, „Produktorientierung“ freilich nur in Ansätzen. Immerhin wird ein Zwischenprodukt in Gestalt einer Zeichnung erstellt.

Zum Aufgabensinn zählt auch die Bestimmung der beteiligten Kompetenzen. Mit Blick auf das Kompetenzmodell der Kultusministerkonferenz für die fortgeführte Fremdsprache (Englisch/Französisch) für die Allgemeine Hochschulreife (KMK 2012) intendiert die vorgeschlagene Lernsequenz die Förderung der Text- und Medienkompetenz. Auf grundlegendem Niveau bestimmt das Dokument der Kultusministerkonferenz konkrete Standards (s. ibd.: 20f.), auf die in der nun folgenden Beschreibung der Arbeitsaufträge jedoch nicht explizit rekurriert wird.

Arbeitsblatt 0 wurde in der Vorbereitungsstunde eingesetzt und dient der Hinführung zum Thema Barcelona. Hier ein Auszug aus diesem Arbeitsblatt: ${ }^{26}$

Abbildung 9: Ausschnitt aus Arbeitsblatt 0 der Unterrichtssequenz zu Ne me quitte pas (Winkels 2015)

En un red social encontráis comentarios sobre Barcelona. La pregunta es: ¿Qué os gusta de vivir en nuestra ciudad?

Adriana: Yo estoy muy contenta de vivir en Barcelona. ¡Es fantástica! Aquí tenemos de todo: playa y montaña. Cuando estás aburrido, no es difícil encontrar algo que hacer.

Rosa: Mi barrio es muy bonito. Me gusta pasear por el parque Güell, ver la Sagrada Familia. Pero estoy harto de encontrar a tantos turistas en Barcelona. Las calles están llenas de guiris. Me da mucha pereza porque no se puede pasear tranquilamente.

In den auf dem Arbeitsblatt vorgegebenen Stellungnahmen sind gezielte Vorentlastungen für den in den folgenden vier Stunden zu bearbeitenden literarischen Text enthalten, z.B. :

\section{Sprachliche Vorentlastung}

Estoy harto de (ich habe die Nase voll von)

Me da mucha pereza (es ermüdet mich)

\author{
Thematische Vorentlastung \\ Tantos turistas en Barcelona (so viele \\ Touristen in Barcelona) \\ Las calles están llenas de guiris (die \\ Straßen sind voll von Touristen aus \\ dem Norden)
}

Die folgende Teilaufgabe II bereitet durch die Aufmerksamkeitslenkung auf positive und negative Aspekte ein einfaches mentales Modell für die spätere Textbearbeitung vor: „Haced una lista de los aspectos positivos y negativos de

26 Sprachliche Fehler in den Aufgabenstellungen sind nicht korrigiert, um die Authentizität der Materialien nicht zu verfälschen. 
la ciudad de Barclona que aparecen en los comentarios" (Stellt eine Liste der positiven und negativen Aspekte über Barclona auf, die in den Kommentaren erwähnt werden.).

Teilaufgabe III („José y Robert escriben algo en catalán. Traducid las frases al español. "José und Robert schreiben etwas auf Katalanisch. Übersetzt diese Sätze ins Spanische.) lenkt die Aufmerksamkeit auf das Phänomen catalán (Sprache), das im Comic marginal innerhalb des interkulturellen (Fremd-)Verstehens eine Rolle spielt. Teilaufgabe IV („Escribid las expresiones correspondientes para cada emoticono. "Schreibt die Formulierungen auf, die den einzelnen Emoticons entsprechen.) kann als Ergänzung zu Teilaufgabe II das lernersprachliche Wissen stützen. Teilaufgabe V („Escribe un comentario de 3-4 frases sobre tu ciudad. ¿Qué es lo que (no) te gusta de vivir en tu ciudad?"Schreibe einen Kommentar aus drei bis vier Sätzen über Deine Stadt. Was gefällt Dir (nicht) an Deiner Stadt?) zielt auf die Herstellung eines persönlichen Themenbezugs und auf einen sprachproduktiven Umsatz des bisher Gelernten. Das Arbeitsblatt 0 hat also insgesamt die Funktion einer sprachlichen und inhaltlichen Vorentlastung. Das im Folgenden abgebildete Arbeitsblatt I zielt auf das eigentliche Textverstehen ab.

Abbildung 10: Arbeitsblatt I der Unterrichtssequenz zu Ne me quitte pas (Winkels 2015)

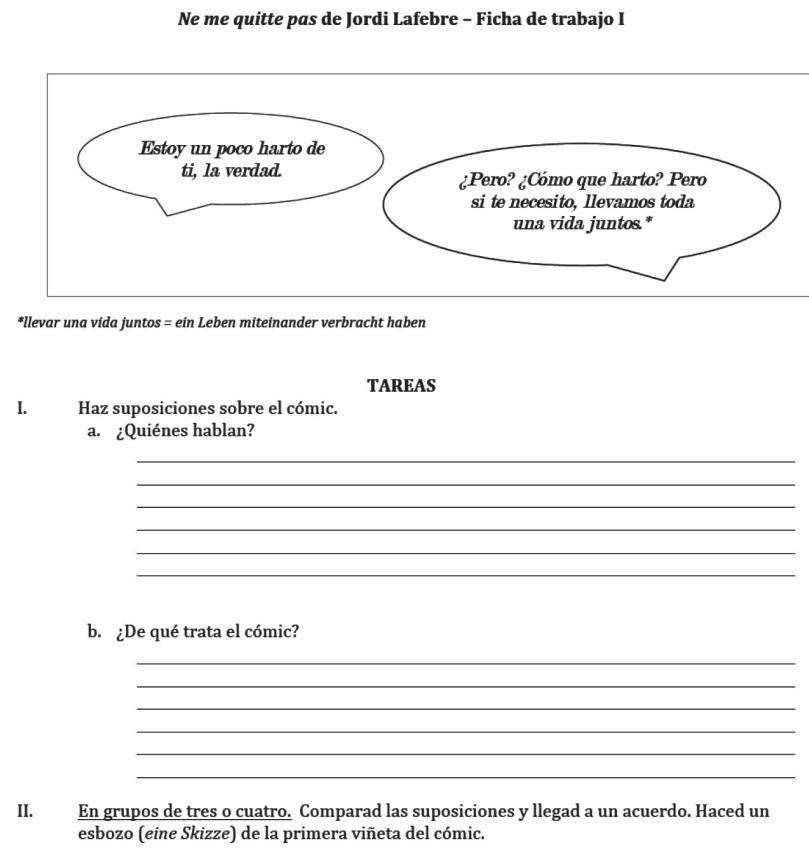


Vor der Arbeit mit dem Arbeitsblatt wird die Aufmerksamkeit der Schülerinnen und Schüler zunächst auf Vorerfahrungen mit dem Genre gelenkt. Sie artikulieren ihre individuellen Leseerfahrungen mit Comics, ihre Vorlieben, ihr Vorwissen. Nebenbei wird comicspezifisches Vokabular eingeführt. Sodann thematisiert die Unterrichtende den Titel Ne me quitte pas, um davon ausgehend von den Lernenden Erwartungen bezüglich der Bedeutung des Titels artikulieren zu lassen. Eine Beschäftigung mit dem Titelbild dagegen ist im vorgeschlagenen Unterrichtsentwurf nicht vorgesehen. Dies könnte Raum für eine mögliche Erweiterung des Aufgabenentwurfs im Verlauf der Aufgabenbearbeitung bieten.

Arbeitsblatt I thematisiert die ersten beiden Bilder des Comics mit dem Ziel, mit Hilfe der thematischen Vorinformationen zu Barcelona sowie der Kenntnis des Titels Vermutungen über die Protagonisten und den Inhalt des Comics anzustellen. Hierdurch kann die Phantasie der Leser angeregt sowie Spannung im Hinblick auf einen Vergleich des vermuteten Inhalts mit dem tatsächlichen Inhalt des Comics aufgebaut werden. Eine daran anschließende Phase der eigenen Bildproduktion ist geeignet, die Spannung auf die im Moment noch vorenthaltenen Bilder des Comics zu erhöhen. Bis zu diesem Zeitpunkt haben die Lernenden noch keinen Blick auf den Comic selbst geworfen, sondern nur mit dem Arbeitsblatt gearbeitet.

Die zeichnerische Lösung der Teilaufgabe Haced un esbozo de la primera viñeta del cómic (Macht eine Skizze zum ersten Bild des Comics) sensibilisiert für den Bildmodus bzw. die eigene visuell-ästhetische Ausdrucksfähigkeit - die Lernenden kennen ja das Bild noch nicht, sondern sollen einen eigenen Entwurf machen - sowie für die visuell-ästhetische Gestaltung durch den Autor bei der anschließenden Lektüre. Damit ist eine weitere Interpretationsleistung durch die Lernenden verbunden.

Die Aufgabe umfasst nun des Weiteren drei Lesephasen des Comics, zwei in der Unterrichtsdoppelstunde und eine als Hausaufgabe. Die Schüler lesen den Comic in Kleingruppen, tauschen sich dabei aus und sichern das Textverständnis durch Beantwortung folgender Fragen: „¿Quiénes son los protagonistas?"(Wer sind die Hauptpersonen?), „¿Qué es el conflicto?" (Worin besteht der Konflikt?), "¿De qué ciudad se trata? ¿Cómo lo sabes?" (Um welche Stadt handelt es sich? Woher weiß Du das?), „¿Qué critica uno de los protagonistas? ¿Cómo reacciona el otro protagonista?" (Was kritisiert eine der beiden Hauptpersonen? Wie reagiert der andere darauf?) und „¿Cómo va a acabar el cómic?" (Wie wird der Comic ausgehen?). Diese Fragen befinden sich bereits auf Arbeitsblatt IIa.

Das rein inhaltsbezogene fragengelenkte Verfahren hat den Vorteil, bei Lesern unterer fremdsprachlicher Kompetenzniveaus ein Basisverstehen $\mathrm{zu}$ sichern, 
ohne den Primärleseeindruck zu beeinflussen. Dieser erste Leseeindruck wird durch die didaktische Reserve Primera impresión in dieser ersten oder der zweiten Doppelstunde abgerufen.

Am Ende der Preguntas de comprensión 1 (Verständnisfragen 1) und vor der Lektüre der letzten Seite des Comic steht eine Frage zum möglichen Ausgang des Comic: ¿Cómo va a acabar el cómic? Die Lektüre der letzten Seite wird folglich bis zur Beantwortung dieser Frage zurückgehalten. Immerhin bieten die beiden letzten Bilder auf Seite fünf Anlass für eine skeptische Aussicht auf die weitere Entwicklung der Beziehung von Stadt und Bewohner. Sie beinhalten eine Zuspitzung der Handlung, denn die Stadt legt ihrem Bewohner nahe, er könne sie ruhig verlassen, wenn er ihrer derart überdrüssig sei.

Mit Arbeitsblatt IIb Preguntas de comprensión 2 werden die von den Lernenden geäußerten Vermutungen aufgegriffen und weiterverarbeitet. Der Aufgabensinn umfasst an dieser Stelle auch eine Auseinandersetzung mit dem katalanischen Satz „N'estic fart" (Ich bin es leid). Ein interkomprehensiver Vergleich mit dem spanischen Estoy harto ist nicht explizit im Verlaufsplan vorgesehen, könnte sich aber im Unterrichtsgespräch ergeben.

Die Lernenden sind sodann aufgefordert, die Unterschiede zwischen ihren Vermutungen und dem tatsächlichen Ende des Comics zu diskutieren: „¿Cómo acaba el cómic? ¿Qué diferencias hay entre el fin real y vuestras suposiciones?" (Wie endet der Comic? Welche Unterschiede gibt es zwischen dem tatsächlichen Ende und euren Vermutungen?). Daran schließt sich die Frage nach der Person auf dem letzten Bild an, eine Aufgabe, die Raum für eigene kreative Lösungen lässt. Die Frage nach der individuellen Rezeption des Comics („Gefällt dir der Comic?") rundet diese Rezeptionsphase ab.

Der Aufgabensinn umfasst weiterhin eine Zusatzaufgabe zur grafischen Gestaltung des Comics (Las imágenes) und einen Lektüreteil verbunden mit einer geschlossenen diagnostischen Aufgabe. Die Aufgabe zur grafischen Gestaltung ist eine interpretatorische Anschlussaufgabe und fokussiert auf die Gestaltung des Hintergrunds, der anwachsend von weiß zu schwarz und wieder zu weiß wechselt und die Wirkung dieses Gestaltungsmittels. Der zweite Teil der Zusatzaufgabe hebt drei Textfragmente aus dem Comic hervor, die die Parallelität des Verhältnisses des Bewohners zu seiner Stadt und des Verhältnisses von Mann und Frau belegen. Die kleinschrittige und stark gelenkte Aufgabenstellung wird durch eine weitere Zusatzaufgabe abgeschlossen: „El hombre en realidad habla con sí mismo. Discutid“ (In Wirklichkeit spricht der Mann mit sich selbst. Tauscht euch darüber aus). Hier wird der Fall antizipiert, dass die Lernenden nicht zu einer plausiblen Deutungshypothese bezogen auf die Hintergrundvariationen 
kommen. Es wird daher eine ganz bestimmte Deutungshypothese zur Diskussion gestellt mit dem Ziel, auf alle Fälle zu einer ergebnisoffenen Diskussion zu kommen und damit gegebenenfalls das Erstverstehen kritisch zu reflektieren. Dies gilt auch für Aufgabenteil II.

In einer weiteren Nachbereitungsphase werden zwei Fragen gestellt: „„Es importante el texto para entender el cómic?" (Ist der Text wichtig, um den Comic zu verstehen?) und „¿Son importantes las imágenes para entender el cómic?" (Sind die Bilder wichtig, um den Comic zu verstehen?). Diese Fragen zeigen ein stark lenkendes Lehrkonzept (vgl. Tesch 2010), das den Fall antizipiert, dass es den Lernenden sowohl konzeptionell als auch zielsprachlich schwer fallen könnte, die Wirkung des Comics zu analysieren. Überdies suggerieren geschlossene Frageformate (¿Quién habla? ¿Quiénes son? ... Wer spricht?, Um wen handelt es sich?) den Lernenden, es gäbe eine bestimmte richtige Antwort, was die Aushandlungsprozesse wiederum auf ein „Ausknobeln“ dieser erwarteten Antwort einengt.

Winkels selbst äußerte sich im Nachhinein kritisch bezüglich der ihrer Meinung nach zu kleinschrittigen Lenkung der Comicbearbeitung und bedauerte, die Sequenz vor der eigentlich literarästhetischen Arbeit am Comic aus Zeitgründen beendet zu haben. Offenere Aufgabenstellungen hätten selbstverständlich zu anderen Ergebnissen geführt, und literarästhetisch orientierte produktive Aufgabenstellungen, wie z.B. die Herstellung eines eigenen Comics, hätten gegebenenfalls stärker zu einem kreativen Erleben geführt, das sowohl auf sprachlicher als auch auf soziokultureller Ebene Lernzuwächse ermöglicht, die durch die eingesetzten rezeptiv-reproduktiven Aufgaben nicht zu erzielen sind. Der Aufgabensinn liegt somit auch weniger in der literarischen Anschlusskommunikation als vielmehr in der sozialen Aushandlung des Leseverstehens eines spanischen Comics.

Es folgt nun der Übergang zur Analyse der Aufgabenbearbeitung, d.h. der Ermittlung des kommunikativen und des dokumentarischen Sinns in der Aufgabenbearbeitung. Zunächst wird jedoch in einem Exkurs zum bereits erwähnten Forschungsseminar auf typische Herausforderungen bei den ersten Erfahrungen mit der Anwendung der Dokumentarischen Methode eingegangen.

\subsubsection{Vom Was zum Wie: Wechsel der Analyseeinstellung}

Die drei Sitzungen des fachdidaktischen Forschungsseminars im Wintersemester 2015-2016, die dem Comic Ne me quitte pas gewidmet waren, sollten der Vertrautwerdung mit der rekonstruktiven Fremdsprachenforschung dienen. Vorausgegangen waren drei Sitzungen mit theoretischem Input zur rekonstruktiven 
Unterrichtsforschung. In Vorbereitung der vierten Seminarsitzung hatten die Studierenden die Hälfte des ersten Transkripts der ersten Unterrichtsstunde zum Comic thematisch zu gliedern. In der vierten Sitzung wurde dann der Anfang des Transkripts 1 (bis Zeile 85) formulierend und reflektierend in Kleingruppen interpretiert. Den Studierenden war zu diesem Zeitpunkt noch nicht bewusst, dass und wie sich aus der ersten Begegnung mit dem Transkript Rahmenorientierungen ergeben könnten. Die thematische Gliederung zeigte, dass bereits auf den ersten beiden Transkriptseiten eine Reihe von Themen notiert werden konnte, deren weiteres Vorkommen im Diskursverlauf weiterverfolgt werden könnte, z.B. scaffolding bei der Worterschließung oder Wortspiele. Die Seminarteilnehmer konnten dabei ein erstes Verständnis für die Transformation fachdidaktischen Wissens (in Form von Aufgaben) in praktisches Wissen (in Form einer Aufgabenbearbeitung) gewinnen. Die intensive Beschäftigung mit dem Transkriptanfang diente als Interpretationsübung im Hinblick auf den Wechsel vom Was zum Wie der Analyse. Es erfolgte jedoch noch keine Relevanzentscheidung im Hinblick auf auszuwählende Passagen. Im Folgenden werden die vier Seminarübungen zur thematischen Gliederung, zur formulierenden und zur reflektierenden Interpretation sowie zur Fall(teil)beschreibung wiedergegeben sowie einige überarbeitete Ergebnisse.

Als große forschungspraktische Schwierigkeit erweist sich für Novizen im Umgang mit der Dokumentarischen Methode meist der Wechsel der Analyseeinstellung vom Was zum Wie der Verständigung und insbesondere die Feinanalyse der Diskursaufzeichnung Zeile für Zeile. Häufig gelingt es ihnen nicht, aus dem Relevanzsystem der Beobachteten herauszutreten, d.h. ihre Versuche, reflektierend zu interpretieren, unterscheiden sich häufig nicht von der formulierenden Interpretation, sondern gleichen eher einer weiteren Umformulierung. Die Identifikation von Proposition, Ausführung und Konklusion und darauf aufbauend von Vergleichshorizonten gelingt oft nur unter Anleitung.

Im Folgenden wird zunächst auf die thematische Gliederung des ersten Stundentranskripts zur Lernaufgabe Ne me quitte pas näher eingegangen. Wie in Kap. 4.1.3 ausgeführt, lautete die erste Aufgabe, die die Schülerinnen und Schüler bearbeiteten, an Hand der beiden ersten Sprechblasen ohne Bilder Vermutungen über den Comic zu formulieren („Haz suposiciones sobre el cómic“). Die Klasse hatte die schriftliche Arbeitsanweisung, die von Ihnen verlangte, erst in Aufgabe zwei Gruppen zu bilden, offenbar im Einvernehmen mit der Lehrerin dahingehend verändert, dass die Schüler sofort in Gruppen arbeiteten.

\section{Die thematische Gliederung der ersten Unterrichtsstunde}

Die thematische Grobgliederung des ersten Falls zeigt folgende Themen. 
Tabelle 9: Thematische Grobgliederung Gruppe I, Aufgabenbearbeitung I bis III

\begin{tabular}{|ll|}
\hline Zeile & Thema \\
$01-04$ & Vermutungen zum Comic \\
$05-13$ & Vokabelklärung harto (überdrüssig) \\
$14-29$ & Vermutungen zur Situation im Comic \\
$30-39$ & Vermutungen zur Figurenkonstellation im Comic \\
$40-55$ & Verständnisproblem llevar una vida juntos (ein ganzes Leben miteinander \\
& verbracht haben) \\
$56-61$ & Vokabelrecherche zum Wortfeld „Ehe“ \\
$62-64$ & Diskussion zur Thematik des Comics \\
$65-68$ & Vokabelklärung „Ehepaar“ \\
$69-79$ & SuS formulieren Sätze zur Beantwortung der Aufgabe \\
$80-85$ & Erneute Vermutungen zur Figurenkonstellation \\
$86-93$ & Vokabelklärung „Ehemann“ \\
$94-98$ & Erneute Formulierungsversuche \\
$99-102$ & Identifikation der Aufgabenstellung \\
$103-109$ & Schüler versuchen Situation nachzuvollziehen \\
$110-118$ & Alternativvorschlag zur Formulierung der Antwort \\
$119-125$ & Vokabelrecherche,Ehestreit“ \\
$126-132$ & Beendung der ersten Teilaufgabe und Entschluss fortzufahren \\
$133-203$ & Anfertigen der Zeichnung und Unterhaltung darüber \\
$204-233$ & Verhandlungen zur Figurenkonstellation in der Zeichnung \\
$234-236$ & Nachahmung der Figuren \\
$237-249$ & Beendung der Zeichnung \\
$249-253$ & Überlegungen zur Präsentation der Ergebnisse \\
254 & Lehrperson fordert zur Präsentation auf \\
$255-260$ & Schüler beeilen sich fertig zu werden \\
$261-268$ & Vermutungen zur Situation im Comic und zur Frage, um welche Stadt es sich \\
$269-285$ & handelt \\
\hline $286-325$ & Vermutungen zur Antwort auf die Fragestellung und Formulierungsversuche \\
$325-327$ & Beurteilung des Verstandenen \\
$328-343$ & Formulierungsversuche \\
$344-345$ & Beendung der Teilaufgabe \\
$346-358$ & Identifikation und Bearbeitung der Aufgabenstellung \\
$359-364$ & Überlegungen zur Beantwortung der Aufgabe \\
$365-394$ & Formulierungsversuche und zwischendurch Abschweifen vom Thema \\
$395-404$ & Gespräch über Uhrzeit und Buntstifte \\
\hline
\end{tabular}


Die Hervorhebungen lassen erkennen, dass bei der Aufgabenbearbeitung Vokabelklärungen im Vordergrund der Aushandlungsprozesse standen, gefolgt von Vermutungen zu Personenkonstellation und Formulierungsversuchen.

Nach dem Anfertigen der Grobgliederung kann eine thematische Feingliederung derjenigen Passagen vorgenommen werden, die sich durch besondere interaktive Dichte auszeichnen. Die Anfänge der Gruppenarbeit beispielsweise zeigen, wie sich die Rollenverteilung in den Gruppen sehr früh konstituiert.

$\mathrm{Zu}$ Beginns des zweiten Falles, d.h. der zweiten beobachteten Gruppe, wurden folgende Themen verhandelt, die hier zum Vergleich mit Gruppe I vorgestellt werden:

\section{Tabelle 10: Thematische Gliederung einer Themenaushandlung im Unterricht}

$\begin{array}{ll}\text { 001-004 } & \text { Hypothesenbildung zur Teilaufgabe a } \\ 005-013 & \text { Worterklärung zu harto (überdrüssig) } \\ 014-035 & \text { Hypothesenbildung zur Situation } \\ 036-038 & \text { Allgemeine Aussagen zu geschlechtstypischen Eigenschaften } \\ 038-041 & \text { Hypothesenbildung zur Situation } \\ 042-055 & \begin{array}{l}\text { Bedeutungsaushandeln zur Kollokation llevar toda una vida juntos } \\ \text { (ein ganzes Leben miteinander verbracht haben) }\end{array} \\ 056-079 & \text { Satzkonstruktionsprozesse } \\ 080(-126) & \text { Weiterarbeit an Teilaufgabe b } \\ 99-117 & \text { Aushandlung zum Thema Streitgespräch ja oder nein }\end{array}$

Bereits hier gibt ein Vergleich mit der anderen Gruppe erste Hinweise auf Unterschiede: Gruppe zwei schreitet wesentlich zielorientierter im Sinne der Aufgabenerledigung voran, und sie wendet sich bei Verstehensschwierigkeiten kein einziges Mal an die Lehrerin. Sie konsultiert stattdessen ein Wörterbuch. Mit anderen Worten, die Rahmung durch das Aufgabenkonzept ,Lernaufgabe' wird durch die beiden Gruppen unterschiedlich gefüllt. Während Gruppe I sich an Aufgabenerfüllung (z.B. Wortbedeutungsaushandlung, Personenkonstellationen) orientiert, orientiert sich Gruppe II stärker an Aufgabenerledigung.

An Hand dreier ausgewählter Episoden aus dem ersten Stundentranskript zur Aufgabenbearbeitung durch Gruppe I wird nun eine formulierende Interpretation vorgestellt. 


\section{Formulierende Interpretation}

Episode $1^{27}$

1 Paul Ähm ¿Qué significa supi (.) suposiones?

2 Leh Ähm, Vermutungen (.) Laaa-Las suposiciones son Vermutungen anstellen,

3 also hacer suposiciones.

4 Paul ${ }^{\circ} \mathrm{Wei} ß t \mathrm{t}$ du was Vermutung auf Englisch heißt?

5 Marc ${ }^{\circ}$ Supition, nee, suspition, assumption ${ }^{\circ}$

6 [Schreibgeräusche der Kreide an der Tafel]

Paul eröffnet die Aufgabenbearbeitung auf Spanisch mit einer laut gesprochenen Frage nach der Bedeutung des Wortes suposiciones (Vermutungen). Die Lehrerin reagiert auf diese Frage zunächst mit einer Übersetzung ins Deutsche und anschließend einer spanisch-deutschen Worterklärung, die sie in die entsprechende Kollokation überführt. Paul fragt Marc leise nach der englischen Übersetzung. Marc antwortet in drei Ansätzen.

\section{Episode 2}

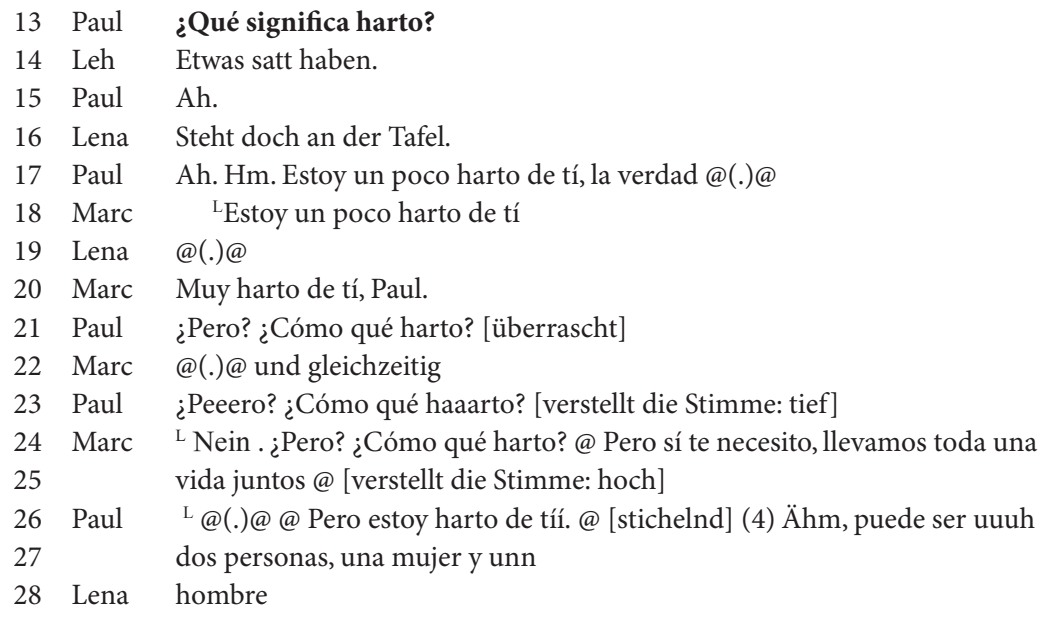

27 Auf eine deutsche Übersetzung wurde an dieser Stelle wie auch bei den folgenden Transkripten verzichtet, da die Übersetzung die Besonderheiten der Lernersprache ausblenden würde und viele Passagen in der Interaktion selbst übersetzt bzw. erklärt werden. 
29 Paul hombre

30 Lena Sí.

31 Paul Y él (.) dice a ella

32 Marc L Streiterio

33 Lena@(.)@

34 Paul@Sí.@ ellas están streitericos@(2)@

35 Lena@(.)@

Paul fragt laut auf Spanisch nach der Bedeutung des Wortes harto und spricht so laut, dass es die Lehrerin hört. Die Lehrerin antwortet mit einer Übersetzung, woraufhin Lena Paul darauf aufmerksam macht, dass diese Übersetzung bereits an der Tafel steht. Paul und Marc lesen nun spontan, teilweise lachend und mit vertauschten Rollen die beiden ersten Sätze des Dialogs im Wechsel. Sie probieren unterschiedliche Betonungen aus, so dass dadurch verschiedene Bedeutungsnuancen durchgespielt werden. Lena schaltet sich erst wieder bei Pauls Suche nach dem Wort "hombre" ein. Paul und Marc schließen mit einem Wortspiel zu einer Formulierung für „streitlustig“ an.

\section{Episode 3}

55 Marc Llevar una vida juntos - ein Leben lang miteinander verbringen

56 Paul Nee, eigentlich nich. Ähm llevar una vida juntos significa ein Leben

57 miteinander verbringen, no?

58 Leh Es que, sí, es presente, also es ist eigentlich Präsens, das Ding is aber es ist ne

59 Konstruktion mit llevar una vida juntos, sí, significa que, dass es sie’s schon

60 verbracht haben

61 Paul Ohhh.

62 Lena Ha.

63 Marc ${ }^{\mathrm{L}}$ Aber sie sagt, wenn ich dich brauche?

64 Paul

65 Leh

${ }^{\mathrm{L}}$ pero necesitoo es presento no?

66 Ende is, sondern bis jetzt haben sie schon das Leben miteinander verbracht

67 Paul Aber heißt das dann, als ich dich gebraucht habe oder wenn ich dich brauche?

68 Leh Pero si te necesito. Also es ist Präsens.

69 Paul

70 Leh

71 Paul

Lwenn ich dich brauche.

${ }^{\mathrm{L}}$ Genau.

72 Leh

Haben wir ein Leben zusammen verbracht.

Aber es is äähhh ah. Genau. Aber das sí ist nicht das si von wenn, sondern im bestätigenden Sinne, also von ja. Also das ist ne Satzkonstruktion äh, die bedeutet, 
80 Leh ich brauche dich, wirklich. Also die das quasi nochmal unter streicht. Sí im Sinne von ähm (.)

$\mathrm{Ja}$.

L Dringend.

Ich brauche dich. Wir verbringen ein Leben miteinander.

Wir haben ein Leben lang miteinander verbracht. Bis jetzt schon. Also wir haben diese Zeit miteinander verbracht. So, ne?

Marc und Paul verständigen sich über die Bedeutung der Kollokation „llevar una vida juntos". Paul wendet sich jedoch wieder laut und vernehmlich an die Lehrerin, um Marcs Antwort bestätigen zu lassen. Die Lehrerin geht in einer gemischt spanisch-deutschen Erklärung näher auf die Bedeutung der Wortverbindung ein. Doch Marc fragt noch einmal nach. Es entstehen Unsicherheiten bezüglich des Tempusgebrauchs und des verwendeten Adverbs sí. Die Lehrerin unternimmt einen zweiten Erklärungsversuch und nach einem Übersetzungsversuch Pauls einen weiteren Erklärungsansatz, diesmal fokussiert auf die Bedeutung der Konjunktion si. Paul und Marc greifen diesen Erklärungsansatz in Übersetzungsversuchen auf. Die Lehrerin bemüht sich um eine Gesamtübersetzung, die noch einmal den Tempusgebrauch erhellen soll.

An die formulierende Interpretation schließt der Arbeitsschritt der reflektierenden Interpretation an, in dem nun der Wechsel der Analyseeinstellung vom Was zum Wie der Interpretation durchgeführt wird. Es werden im Folgenden Interpretationsbeispiele für die drei oben vorgestellten Episoden des Bearbeitungsgesprächs in Gruppe I gegeben.

\section{Reflektierende Interpretation}

Episode 1: Öffentliches und nicht öffentliches Bedeutungsaushandeln unter Nutzung von Sprachvergleichen

Erste Proposition:

Paul fragt auf Spanisch nach der Bedeutung des Wortes „suposiciones“ und initiiert damit eine Wortschatzklärung im Fragemodus. Er artikuliert die Frage laut und an die Lehrerin gerichtet. Das neue Wort scheint ihm phonetisch noch nicht vertraut zu sein, was sich darin äußert, dass er zwei fehlerhafte Aussprachevarianten verwendet. 
Vorläufige Konklusion und erste Orientierung:

Die Lehrerin antwortet spontan und in normaler Lautstärke auf Deutsch. Sie konkludiert das Thema zunächst, indem sie die Frage direkt, aber nicht zielsprachlich beantwortet. Nach einer kurzen Pause setzt sie jedoch ihre Konklusion noch einmal aus, um ihre Antwort instruierend und in erhöhter Lautstärke in einen vollständigen spanischen Satz mit deutschem Einschub einzukleiden.

Sie signalisiert damit ihr Ziel, die Aufgabenbearbeitung grundsätzlich zielsprachig zu führen und elaboriert gleichzeitig ihr Feedback, indem sie die für Paul problematische Vokabel erstens phonetisch korrekt wiederholt, sie zweitens mit dem Artikel versieht und sie drittens in die Kollokation "Vermutungen anstellen" einbindet, die sie viertens auf Spanisch formuliert. Damit verbindet sich die Erwartung, dass ihr Input verstanden wird.

Transposition und Gegenhorizont:

Paul hebt die Themenkonklusion im Flüstergespräch mit Marc wieder auf. Das heißt, das Thema ist für ihn noch nicht abgeschlossen, sondern löst die emergente Frage nach der englischen Bedeutung aus. Er führt diese Themenverlagerung in die Sphäre der Kleingruppe zurück. Seine Frage wird von ihm zum nicht öffentlichen Thema deklariert, zum Flüsterthema, das nur ihn und Marc etwas angeht.

Orientierung: nicht öffentliche Aufgabenbearbeitung unter Einbeziehung des Englischen

Pauls Orientierung besteht in einer Trennung von öffentlicher und nicht öffentlicher Aufgabenbearbeitung. In letzterer darf auch ein Exkurs ins Englische erfolgen, was wiederum darauf schließen lässt, dass Marc davon ausgeht, sein Exkurs könne das öffentliche einsprachige, allenfalls deutsch-spanisch-bilinguale Unterrichtsskript stören. Der Vergleich mit dem Englischen verweist auf seine Sprachbewusstheit, er sucht ein englisches, scaffold: Das Englische spielt bei ihm mithin die Rolle einer aktiven Lernfolie oder Lernhilfe, ihre Nutzung kollidiert jedoch in seinen Augen mit dem offiziellen Unterrichtsskript.

Konklusion:

Marc elaboriert und konkludiert das Flüsterthema im Modus einer tentativen Antwortkette. Er bleibt im Flüstermodus und antwortet zunächst mit dem Wortbildungsversuch „supition“, den er im phonetischen Monitor sofort als korrekturbedürftig interpetiert, mit "nee“ verwirft, durch „suspition" ersetzt, das er wiederum als semantisch korrekturbedürftig interpetiert und schließlich durch „assumption“" ersetzt. 
Aspekt Sprachbewusstheit:

Auf der lernersprachlichen Ebene zeigt diese Episode erste Markierungen der individuellen, zone of proximal development' (ZPD). Für Paul liegt das Wort suposiciones innerhalb seiner ZPD, es kann hier durch Lehrerhilfe erschlossen werden. Vor allem zeigt aber seine Kenntnis ähnlicher englischer Wörter, dass er die mit suposiciones verbundene linguistische bzw. begriffliche Abstraktion kennt.

Episode 2: Sinnkonstruktion unter Nutzung von Wortspielen

Methodischer Exkurs: Interaktive Dichte und Fokussierungsmetapher

Bereits bei der ersten Durchsicht des Transkripts weckte Marcs spaßige Wortbildung "Streiterio“ die Aufmerksamkeit der Teilnehmer des erwähnten Forschungsseminars. Die Gruppe, die diese Episode bearbeitete, merkte an der heiteren Aufgabenbearbeitung ab Zeile 17, dass das Thema Wortspiele in einen größeren Rahmen einzuordnen sei. Die Episode schließt unmittelbar an die oben interpretierte Episode an und wird durch die Suche nach der Wortbedeutung von harto (überdrüssig) ausgelöst.

Rituelle Themeninitiierung im Fragemodus und Gegenhorizont Pauls:

Wieder ist es Paul, der die automatisierte Frageformel „Qué significa ...?" einsetzt. Nach mehrmaligem Fragen in der Kleingruppe, das dort nicht zur Lösung führt, fragt Paul schließlich laut vernehmlich die Lehrerin (13). Pauls Gegenhorizont zur Aufgabenbearbeitung in der Kleingruppe besteht darin, der Lehrerin die Rolle der Expertin zuzuweisen, die bei lexikalischen Schwierigkeiten anzurufen ist und der Kleingruppe somit die Expertise zur Lösung dieser Schwierigkeiten abzusprechen. Paul verlässt auf diese Weise den gemeinsamen Rahmen der Aufgabenbearbeitung in der Kleingruppe.

\section{Thematische Konklusion und Ratifizierung:}

Die Lehrerin konkludiert das Thema der Bedeutungserschließung wieder wie bereits in Episode eins durch eine Übersetzung ins Deutsche. Paul ratifiziert sein Verständnis („,ah") und Lena verweist auf die Tafel, wo offenbar bereits einige Worterklärungen notiert sind.

Methodischer Exkurs zur interaktiven Dichte und Orientierung an Rollen: Mehrfach sind im Transkript Passagen vorhanden, in denen gelacht wird. Die entsprechende Symbolik im Transkript und das anschließende Validieren durch die Tonspur brachte die Seminarteilnehmer nun auf die Interpretation, dass sich hier (17 bis 25) ein Rollenspiel entfaltet, in dem Marc und Paul die Rollen der beiden Streitenden einnehmen. Das Aushandeln der Bedeutung wird zu einer 
Identifikation mit Rollen, d.h. es kommt zu einer Orientierung an Rollenidentifikation. Die Arbeit am Sprachverstehen tritt völlig in den Hintergrund zugunsten der Sprachproduktion, ja der Sprachvariation im Rollenspiel mit Stimmenverstellen nach oben und unten und stichelndem Ton.

Paralleler und univoker Modus:

Die völlige Kongruenz der Rahmungen zeigt sich diskursanalytisch auch im parallelen Modus der Äußerungen. Marc und Paul übernehmen jeweils den fragend vorgetragenen Ausruf „Cómo qué harto?" und variieren nur die Stimme. Zwischen Lena und Paul kommt es schließlich sogar zur univoken Äußerung "hombre“ als Nachsprechen des von Paul begonnenen Satzes „Ähm, puede ser uuuh dos personas, una mujer y unn".

Aspekt Sprachbewusstheit:

Das Rollenspiel kann lernersprachlich als Aneignungsprozess gedeutet werden, in dem gedankenexperimentell mögliche Kontexte konstruiert werden. Dies führt zu Pauls Schluss, dass es sich um einen Streit zwischen Mann und Frau handelt (26-27). Lena ergänzt „hombre“ (28) und validiert auf diese Weise Pauls Deutung. Marc konkludiert schließlich die Episode durch die Bedeutungssynthese "Streiterio" (32), die wiederum von Lena durch ein Lachen ratifiziert und von Paul durch die Elaboration "ellas están streitericos" lachend validiert wird. Auch hier wird wieder mit Sprache gespielt, ein deutscher Stamm bekommt mögliche spanische Endungen, was zwar auf eine Art Verballhornung hinausläuft, gleichzeitig eine besondere, ludische Form von Sprachbewusstheit impliziert.

Episode 3: Unterrichtenden-scaffolding zu semantischen Fragen

(gekürzte Interpretation)

Verfestigung des Gegenhorizonts „Auflösung der Aufgabenbearbeitung in der Kleingruppe":

Paul und Marc rätseln weiter an der Bedeutung der Sprechblase. Marc initiiert eine Proposition im Modus einer Übersetzung vom Spanischen ins Deutsche. Paul verlässt jedoch die Orientierung an der gemeinsamen Aufgabenbearbeitung, indem er wieder laut die Lehrerin als Auskunftsquelle nutzt. Er bittet sie um Validierung des Übersetzungsversuchs. Sie nimmt auch diesmal die attribuierte Rolle an und elaboriert eine längere Antwort auf Deutsch. Sie nimmt damit verstärkt die Expertenrolle ein und gliedert sich phasenweise in die Gruppe ein. 
Themenentfaltung im Rahmen expliziter Sprachbewusstheit; Homophonie und Semantik als Probleme:

Paul und Lena ratifizieren das Verständnis von "llevar una vida juntos", Marc aber schließt mit einer überlappenden Proposition im Modus einer Frage an. Er thematisiert das Verständnis des Satzes "pero si te necesito". Paul elaboriert Marcs Frage, indem er das Präsens des Verbs als Frage in den Raum stellt. Es entfaltet sich nun ein längerer Dialog zwischen Paul und der Lehrerin, in welchem Paul merkt, dass das Problem nicht die Zeitform ist, sondern die Semantik des Adverbs sí. Der Lehrerin erschließt sich erst nach einigen interimären Diskursbewegungen, dass das Verständnis am Adverb sí hängt, das sie selbst allerdings zunächst unpräzise mit „wirklich“ übersetzt anstatt mit „doch“.

Aspekt Sprachbewusstheit:

Die Episode ist durch die Dominanz lernerseitiger Verstehensprobleme gekennzeichnet. Das homophone sí/si hat die Bedeutungen „doch“ und „wenn/falls“. Die Homophonie verursacht ein Verstehensproblem, das die Lehrerin nicht direkt aufzuklären vermag. Ihre Erklärungen bleiben auf der Ebene der Satzbedeutung. Hilfreich wäre eine Erklärung auf der Wortebene gewesen. Auch das Verständnis der Kollokation "llevar una vida juntos" wird dadurch zunächst behindert, dass eine lehrerseitige explizite Instruktion ausbleibt bzw. von ihr mühsame Umschreibungsversuche auf Deutsch vorgenommen werden. Das ,scaffolding' führt zwar letztlich zum Ziel, jedoch bleibt ungeklärt, ob die Schüler für die Semantik von si und sí und den idiomatischen Charakter von "llevar una vida juntos" sensibilisiert werden konnten. Der Lernerfolg dieser potenziell sprachbewusstheitsfördernden Aufgabe bleibt offen, da die beiden sprachlichen Phänomene die Kleingruppe noch überfordern. Sie liegen noch jenseits ihrer ZPD.

Zusammenfassung: Der Wechsel der Analyseeinstellung vom Was zum Wie zeigt hier, wie auch bei der ersten beobachteten Episode, Pauls Orientierung an der direkten Befragung der Lehrerin als Problemlöserin für lexikalische und semantische Fragen, statt die Aufgabenbearbeitung in der Kleingruppe voranzutreiben. Die Lehrerin geht auf diese Orientierung ein, allerdings im Modus teilweise ausschweifender Elaborationen zunehmend auf Deutsch. Es zeigt sich daran eine Unsicherheit bei der Einschätzung der ZPDs in der Kleingruppe. Ihr Bemühen, mit ihren Erklärungen in der Zielsprache zu bleiben, scheitert zunehmend, je komplexer die sprachlichen Herausforderungen werden. Damit wechselt die Orientierung in dieser Kleingruppe von der Aufgabenbearbeitung auf Spanisch zur Aufgabenbearbeitung auf Deutsch. Die diagnostischen Schwierigkeiten der Lehrerin könnten auf ihre noch geringe Vertrautheit mit der Klasse zurückgehen. 


\subsubsection{Fallvergleich und Typenbildung}

In diesem fünften und letzten Kapitel zur Dokumentarischen Analyse der Leseverstehensaufgabe Ne me quitte pas werden die Arbeitsschritte des Fallvergleichs und der Typenbildung vorgestellt. Es wird auf zwei vorliegende Fallbeschreibungen (Auszug aus Winkels 2015, gekürzt; längere Belegzitate wurden aus Platzgründen nicht berücksichtigt.) zurückgegriffen und diese zunächst zur Illustration der Textsorte ,Fallbeschreibung vorangestellt. Anschließend werden Herausforderungen der Typenbildung diskutiert.

\section{Fall 1}

Die Kleingruppe setzt sich aus Paul, Marc und Lena zusammen. Die erste bearbeitete Passage zeigt, wie Konkurrenz und Kooperation auf der Peerebene Hand in Hand die Progression der Bedeutungsaushandlung fördern können. Die zweite bearbeitete Passage lässt erkennen, dass die hohe Text- und Aufgabenorientierung der Gruppe trotz einer kritischen Situation hinsichtlich der Peer-Konkurrenz eine progressive Aushandlung ermöglicht.

Alle Orientierungen dieses Falls ordnen sich der Aufgabenorientierung unter. Die Gruppe arbeitet sich stets zielstrebig an der jeweiligen Aufgabe ab. Obwohl auch Peer-, Selbstwirksamkeits-, Text- und Fremdsprachenorientierungen eine große Rolle spielen, entfalten sie sich alle unter der Dachorientierung an den Aufgaben. Alle Gruppenmitglieder lassen sich auf den fachlichen Gegenstand und den durch ihn gestellten Arbeitsauftrag hinsichtlich einer Bedeutungsaushandlung ein. Es konstituiert sich durch ein gegenseitiges Bezugnehmen der SuS ein konjunktiver Erfahrungsraum, in dem die Gruppe interagiert. Es zeigt sich des Weiteren eine Rollenverteilung innerhalb der Gruppe, die auf das jeweilige Selbstwirksamkeitskonzept zurückgeht. Paul nimmt die Rolle der Führungsposition ein. Er hat fremdsprachlich das höchste Niveau in der Gruppe und wird von Marc als Experte für Vokabelfragen herangezogen.

Die Gruppe zeichnet insgesamt eine ambivalente Orientierung hinsichtlich der Fremdsprache aus. Die mündliche Kommunikation auf Spanisch hängt bei diesem Fall stark vom Thema ab. Nur wenn eng an der Aufgabe und mit dem Comic gearbeitet wird, spricht die Gruppe Spanisch. Die Bedeutungsaushandlung der Lesart von Ne me quitte pas wird von der Gruppe bspw. als geeignetes Setting angesehen, um auf Spanisch zu kommunizieren. Spanisch wird in vielen Passagen des Falls auch selbst Gegenstand des Gesprächs. Paul stolpert z.B. über die Konstruktion llevar una vida juntos, die zwar auf Spanisch im Präsens formuliert ist, aber eine Bedeutung in der Vergangenheit hat. Die Fremdsprache wird Gegenstand des Gesprächs und verursacht eine thematische Ablösung der inhaltlichen Bedeutungsaushandlung. Andererseits wird der bejahende Partikel sí mit der bedeutungsabweichenden Konjunktion si verwechselt. Paul zieht als Übersetzungshilfen in einem ersten Schritt die Vokabelangabe auf dem Arbeitsblatt, dann die Gruppenmitglieder und als letzte Instanz mich als Lehrerin heran. Innerhalb der Gruppe wird die Strategie verfolgt, einzelne Teile des Satzes Pero si te necesito, 
llevamos toda una vida juntos zu übersetzen und so den Gesamtsinn zu erschließen (Transkript Fall 1: Z.43-82)

Rückschlüsse auf die Sprachbewusstheit und die Lernersprache können insbesondere hinsichtlich des strategischen Repertoires der Gruppe gezogen werden, das dazu dient, den Comic zu verstehen und das Gespräch auf Spanisch zu bewältigen. Paul sucht Analogien zum Englischen (Transkript Fall 1: Z. 4 „Weißt du was Vermutung auf Englisch heißt?"). Marc und Lena kreieren eigene Wörter, zumeist ein deutsches Wort mit spanischem Suffix oder in spanischer Aussprache und vermeiden so stellenweise den vollständigen Rückfall ins Deutsche. So nutzt Marc „Barto“ als spanische Übersetzung für Bart und Lena „Monumenticum“ als spanische Übersetzung für Monument (ebd. Z. 32, 377). Marc und Paul übersetzen ausgehend von der kleinsten Satzeinheit, dem Wort, Satzteile, um einen spanischsprachigen Text inhaltlich zu verstehen (ebd. Z. 43-55).

Die fremdsprachlichen Hilfsmittel lassen sich in eine Verfahrenshierarchie einordnen. Zunächst wird probiert, die fremdsprachliche Leerstelle über kreatives Experimentieren oder Hinzuziehen einer anderen Fremdsprache zu füllen. Gelingt dies nicht, wird sie der Gruppe zur Abarbeitung freigegeben. Führt auch dies zu keinem zufriedenstellenden Ergebnis, wird die Lehrerin in letzter Instanz als fremdsprachliche Expertin konsultiert. Ein etwas anderer Umgang mit der Fremdsprache Spanisch zeigt sich in Fall 2, in dem die dominante Präsenz anderer Orientierungen die Handlungen der Gruppe hervorbringen.

\section{Fall 2}

Die Kleingruppe setzt sich aus Sara, Mia, Sophie und Arne zusammen. Zwei Passagen wurden ausgewählt, da sie interaktiv sehr dichte Sequenzen dokumentieren, bei denen sich alle Gruppenmitglieder am Gespräch beteiligen. Beide Passagen zeigen, wie die hohe Aufgaben-, Text- und Peerorientierung der Gruppe das Fundament für eine gelungene Bedeutungsaushandlung legt.

Die Gruppe zeichnet eine hohe Aufgaben- und Textorientierung aus. Diese äußern sich durch Sophies, Mias und Saras Zielstrebigkeit in der Aufgabenbearbeitung, die sie gleichberechtigt vorantreiben. Der lebens- und realweltliche Bezug des Textes wird immer wieder gesucht, indem inhaltliche Kausalzusammenhänge der Lesarten auf subjektive Sinnhaftigkeit untersucht und diskutiert werden. Der Gesamtzusammenhang der Textstellen wird über Detailverstehen erarbeitet, indem teilweise Wort für Wort übersetzt wird (Transkript Fall 2: Z. 42-54, 294-327).

Die Gruppe weist hinsichtlich der mündlichen Kommunikation auf Spanisch eine niedrige Fremdsprachenorientierung auf. Das Gespräch findet fast ausschließlich auf Deutsch statt. Nur wenn Antworten formuliert werden, wird Spanisch produktiv verwendet. Die Aufgabe wird nicht als Sprachübung für mündliche, wohl aber für die schriftliche Produktion verstanden. Dem liegt auch eine Gewohnheitsorientierung zugrunde, die sich in der Vorgehensweise der Aufgabenbearbeitung in der Gruppe manifestiert. Zum einen kann das Prinzip des Aushandelns auf Deutsch und der Formulierung der Antworten auf Spanisch als in der Klasse gewohnte Vorgehensweise identifiziert werden, zum 
anderen die Aushandlung einer gemeinsamen Antwort, die erst mündlich besprochen und dann verschriftet wird. So werden lernersprachliche Produktionen in der Gruppe gemeinschaftlich ausgehandelt.

Diese Vorgehensweise weist wiederum auf eine andere starke Rahmung der Gruppe hin: die Peerorientierung, die auf Harmonie und demokratische Kooperation ausgerichtet ist. Wie in den oben zitierten Passagen beschrieben, gelingt es der Gruppe so erfolgreich zu kommunizieren und produktiv miteinander zu arbeiten. Handlungen, die dazu verfolgt werden, sind das Einlenken bei Meinungsverschiedenheiten und das $\mathrm{Zu}$ rücknehmen eigener Bedürfnisse. Welches Handeln hervorgebracht werden kann, wenn Peer- und Selbstwirksamkeitsorientierung aufeinandertreffen, wird exemplarisch in der folgenden Fallbeschreibung dargestellt.

Die zitierten Fallbeschreibungen sind jeweils sehr kohärente und kohäsive Texte mit fallverbindenden Bezugnahmen. Es entsteht auf diese Weise ein anschauliches Bild der Lerngruppen bei ihrer Bearbeitung der Lernaufgabe Ne me quitte pas. Die Konjunktivität im Erfahrungsraum des Unterrichts und der Aufgabenbearbeitung wird an Hand der herausgearbeiteten Orientierungen sehr plastisch, die ihrerseits Grundlage für den Fallvergleich und die auf ihm beruhende Typenbildung sind.

Winkels ordnet ihre Interpretationsergebnisse fünf Fällen zu, indem sie die vier beobachteten Gruppen jeweils als Fall definiert. Einen fünften Fall bildet in ihrer Studie die Gruppendiskussion, die separat nach Abschluss der Aufgabenbearbeitung mit dem Ziel einer Gesamtevaluation der Sequenz durchgeführt wurde. Der fünfte Fall wird als nicht homolog bezeichnet und daher als externes Validierungsinstrument verwendet (Winkels 2015: 35). Hinzuzufügen wäre, dass nur jeweils zwei beschriebene Fälle direkt vergleichbar sind, da nur dort dieselben Aufgaben bearbeitet wurden.

Winkels ermittelte folgende dominanten Orientierungen während der Aufgabenbearbeitung (ibd.: 37): 
Abbildung 11: Orientierungen bei der Aufgabenbearbeitung des Comics Ne me quitte pas im Spanischunterricht (Winkels 2015: 37)

\begin{tabular}{|l|l|}
\hline \multicolumn{1}{|c|}{ Orientierung } & Äußert sich in den Fällen dieser Arbeit bspw. in... \\
\hline Aufgabenorientierung & $\ldots$ intensiven Bedeutungsaushandlungen \\
\hline Ergebnisorientierung & $\ldots$ der Formulierung gemeinsamer Antworten \\
\hline Textorientierung (Ne me quitte pas) & $\ldots$ der Herstellung lebensweltlicher Bezüge \\
\hline Fremdsprachenorientierung & $\ldots$ der Kommunikation auf Spanisch \\
\hline Peerorientierung & $\ldots$ demokratischer Kooperation \\
\hline Selbstwirksamkeitsorientierung & $\ldots$ der Ausführung von Rollen \\
\hline Gewohnheitsorientierung (Methoden, Material) & $\ldots$ der Vorgehensweise in Kleingruppenarbeit \\
\hline
\end{tabular}

Sie generierte aus den Interpretationen folgende sinngenetische Typik:

Abbildung 12: Sinngenetische Typik bei der Aufgabenbearbeitung des Comics Ne me quitte pas (Winkels 2015: 65)

Realisierung von Unterricht

Progression in der

Aufgabenbearbeitung

Thematische Sinnkonstruktion und Relevanzbildung

Progression in der

FremdspracheSpanisch

Prozesssteuerung durch Rollenübernahme
- Subtyp A: Schriftliche Sprachübung

- Subtyp B: Schriftliche und mündliche Sprachübung

- Subtyp C: Kooperation und Konkurrenz

- Subtyp D: DemokratischeKooperation

- Subtyp E: Konträre Positionen

Abgesehen von der geringen Trennschärfe von Merkmalen der Peerorientierung und der Selbstwirksamkeitsorientierung wäre vor allem zu diskutieren, ob es sich hier bereits um eine Typik oder nicht eher um eine Auflistung zentraler Rahmungen handelt. Eine Typenbildung resultiert generell aus einem Fallvergleich. Da hier jedoch alle vier bzw. fünf Fälle lediglich als Variationen innerhalb ein 
und derselben Lerngruppe betrachtet werden, kann man nur eingeschränkt von einem Fallvergleich sprechen. Die Lerngruppe bildet lediglich interne Kontraste (interne Homogenität), externe Kontraste zu anderen Klassen bzw. Lerngruppen (externe Heterogenität) wurden nicht erhoben. Denkbar wäre allerdings, die unterschiedlichen empirisch ermittelten Hierarchien dieser Rahmungen noch einmal den vier Lerngruppen zuzuordnen, denn auf diesem Wege ließe sich ggfs. zwischen einer Typik der beobachteten Lerngruppe A, einer Typik der beobachteten Lerngruppe B, einer Typik der beobachteten Lerngruppe $C$ und einer Typik der beobachteten Lerngruppe D unterscheiden. Lerngruppe A wiederum neigt zu bestimmten Subtypen, Lerngruppe B zu anderen, u.s.w. Die so ausdifferenzierten Hierarchien ließen sich in der Folge als Typiken der Aufgabenbearbeitung in der Gesamtgruppe bezeichnen, wenn auch auf schwacher empirischer Basis. Der Vergleich würde wesentlich auf den verschiedenen Persönlichkeitstypen innerhalb der elfköpfigen Lerngruppe beruhen, was jedoch den Vorteil hätte, die individuelle Sprachbewusstheit angemessen berücksichtigen zu können. Diese wird hier zwar in Form einer Rahmung durch Progression in der Fremdsprache Spanisch mitmodelliert, allerdings als Typus nur ansatzweise herausgearbeitet. Individualtypisch ist bei Paul, Marc und Lena neben dem Bemühen um die Verwendung des Spanischen als Arbeitssprache auch die Nutzung mehrsprachiger Erschließungsstrategien sowie das Spielen mit Sprache als Erschließungsstrategie.

Doch auch bei ausgereifteren Analysen größerer samplings wie bspw. bei Bracker (2015), wo ebenfalls die literarische Anschlusskommunikation den Untersuchungsgegenstand bildete, kann das Zustandekommen einer Typologie auf Probleme stoßen. Dies kann am Forschungsgegenstand selbst liegen - das literarische Textverstehen ist per se durch eine Fülle an Lesarten gekennzeichnet -, vor allem aber an der Verkomplizierung der Interaktionen durch die Sinnebene der Lernersprache. Die Individualität der Lernersprache unterläuft in manchen Fällen die Sozialität der thematisch-inhaltlichen Sinnkonstruktion (vgl. Kap. 2.1) und kann die Bestimmung z.B. milieu- oder geschlechtsspezifischer Typiken verhindern. Dieses Problem wurde von Bracker dadurch zu kompensieren versucht, dass sie die Ergebnisse ihrer Analyse zur literarischen Anschlusskommunikation im Englischunterricht von Oberstufenkursen in theoretischer Anlehnung an Oevermann (1991) post hoc noch einmal mit der dichotomen Deutungshypothese der "reproduzierenden“ und der ,innovativen“ Wirkung von Aushandlungsprozessen abglich. Damit bringt sie einen zusätzlichen theoretischen Horizont in ihren methodologischen Ansatz ein und verlässt die eigentlich praxeologische Rationale ihrer Studie. Der Rückbezug auf eine extern generierte Deutungshypothese ist 
vertretbar, da sie diese für eine kategoriale Zuordnungsprozedur post hoc nutzt und nicht als Bestandteil der reflektierenden Interpretation. Es entsteht auf diese Weise eine dem Gegenstand durchaus angemessene zusätzliche theoretische Verortung im fremdsprachlichen Literaturunterricht auf drei Ebenen, der Ebene der Fremdsprache, der Ebene der inhaltlichen Bedeutungsaushandlung und der interaktionalen Ebene. Diese Verortung kann jedoch nicht über das theoretische Problem einer soziogenetischen Typenbildung hinwegtäuschen, das auf die Komplexität des Untersuchungsgegenstandes und die Individualität der Lernersprachen zurückgeht. Eine Lösung dieser grundsätzlichen Problematik kann nur durch die Mitmodellierung der individuellen Sprachbewusstheit innerhalb der Gesamttypologie gefunden werden. Hier besteht grundsätzlich noch ein erhebliches Desiderat in der Fremdsprachenforschung. In der Datentriangulation mit lernerbiografischen Daten liegt eine zusätzliche Validierungsmöglichkeit.

In diesem Kapitel wurden die Transformation eines literarischen Kurztextes in eine Lernaufgabe für den Spanischunterricht in einer elften Klasse sowie die Arbeitsschritte einer Untersuchung zur Aufgabenbearbeitung selbst - die zweite Transformation - vorgestellt. Die Lernaufgabe zum Comic Ne me quitte pas fokussierte auf das literarische Verstehen ausgehend von einer Kleingruppenarbeit. In den Aushandlungsprozessen zeigten sich verschiedene Sinnkonstruktionsbzw. Aushandlungsprozesse, die zwar Gemeinsamkeiten, aber auch deutliche Unterschiede aufwiesen, so dass es möglich war, erste Hinweise auf eine Typik zu generieren. Diese konnte jedoch auf Grund mangelnder Vergleichsfälle nicht abgeschlossen werden.

Nach dieser Innenperspektive des Fremdsprachenunterrichts (an Hand der Untersuchung der Bearbeitung einer komplexen literarischen Leseaufgabe über mehrere Unterrichtsstunden hinweg) wenden wir uns nun der Außenperspektive auf den Fremdsprachenunterricht zu, der Verständigung über das Fremdsprachenlehren. In der ,Fallwerkstatt' werden dazu narrative Interviews mit zwei Junglehrerinnen betrachtet. Das Lehramtsstudium liegt noch nicht lange zurück, und die erlebte Berufspraxis generiert bereits erste berufsbiografische Orientierungsmuster. Aus forschungsmethodischer Sicht interessiert, wie auch hier beim narrativen Interview der Wechsel der Analyseeinstellung vom Was zum Wie vollzogen werden kann.

\subsection{Die Verständigung über das Lehren von Sprachen. Narrative Interviews mit Bianca und Anne}

Die Interviews mit „Anne“ und „Bianca“, beide Berufsanfängerinnen, deren Referendariat ein Jahr zurücklag wurden im Rahmen eines Forschungsseminars 
gemacht. Die Impulsfragen lauteten, warum sie sich für diesen Beruf entschieden hatten und welche Bilanz sie nach einem Jahr in der Schule zogen. Einschränkend ist vorweg zu erwähnen, dass die exakte Formulierung der Impulsfragen nicht hinreichend abgesprochen wurde, so dass sich durch Fragennuancen gesteuerte Variationen in der Interviewentwicklung ergaben.

\subsubsection{Interview mit Bianca}

Eine Gruppe Studierender hatte sich für die Durchführung eines narrativen Interviews im Rahmen der Erforschung der Verständigung über das Lehren von Sprachen entschieden und eine junge Lehrerin für das Interview angesprochen. Erst später wurde dem Seminarleiter bekannt, dass die angesprochene Person eine gute Bekannte einer der drei Forscherinnen war, allerdings nicht der Interviewerin selbst. Damit war eine der klassischen Regeln für die Durchführung eines narrativen Interviews ansatzweise verletzt, und es stellte sich die Frage, inwieweit die Daten zu nutzen seien. Bei näherer Analyse stellte sich heraus, dass die Bekanntheit Biancas mit einer der beiden Forscherinnen und die gegebenen konjunktiven Erfahrungsräume das Interview zu einer Art implizitem Gruppengespräch hatte werden lassen: Bianca war Berufsanfängerin im zweiten Jahr, hatte also noch einen relativ direkten Bezug zur ersten und zweiten Phase ihrer Ausbildung; die Forscherinnen standen am Ende der ersten Phase ihrer Ausbildung und waren somit nur wenig jünger als Bianca. Das Hochschulstudium die erste Phase der fachdidaktischen Ausbildung - bildete einen gemeinsamen Erfahrungsraum der vier Personen, und das Interview gestaltete sich folglich so, dass zwar nur Bianca redete, sie sich jedoch latent auf die Studierenden als peer group bezog und explizit auf den gemeinsamen Erfahrungsraum des Hochschulstudiums referierte.

Der Umgang mit Daten sollte in der qualitativen Forschung keinem rigiden Schematismus folgen, sondern reflexiv und flexibel auf den Untersuchungsgegenstand reagieren. Strauss \& Corbin (1996) beschreiben den Umgang mit Daten als ein Zusammenspiel von Forscher und Daten:

Analysis is the interplay between researchers and data. It is both science and art. It is science in the sense of maintaining a certain degree of rigor and by grounding analysis in data (Strauss \& Corbin 1996: 13).

Analyse bedeutet das Zusammenspiel von Forschern und Daten. Dieses Zusammenspiel ist sowohl Wissenschaft als auch Kunst. Wissenschaft ist es insofern, als es ein gewisses $\mathrm{Maß}$ an Strenge einhält und die Analyse auf Daten gründet. (Übers. BT) 
In diesem Sinne entschied ich mich, den Fall Bianca in diese Veröffentlichung einzubeziehen, dabei auch die spezifische Beziehung der Interviewten zu den Interviewerinnen mit in die Analyse hineinzunehmen und die impliziten Normalitätsvorstellungen Biancas an der Schnittstelle von pädagogisch-fachdidaktischer Ausbildung und Berufspraxis zu untersuchen.

Das Interview weist sieben inhaltliche Abschnitte und acht Hauptthemen auf. Ein Thema (Abschnitt 6, hervorgehoben) bringt inhaltlich nichts Neues bzw. wiederholt mehr oder minder ein anderes Thema (Abschnitt 2):

Tabelle 11: Narratives Interview „Bianca“, thematische Gliederung

$\begin{array}{lll}\text { Abschnitt } & \text { Zeilen } & \text { Thema } \\ 1 & 01-17 & \text { Biancas Studien- und Berufswahl } \\ 2 & 18-33, & \text { Motivation durch positive Einstellung der Schülerinnen und Schüler } \\ 6 & 145-158 & \text { zu Fremdsprachen } \\ 3 & 34-60 & \text { Motivation durch erfolgreiche Umsetzung ihres Konzepts } \\ 4 & 64-84 & \text { Freiräume neben der Lehrwerksarbeit } \\ 5 & 88-141 & \text { Didaktische Theorie und Unterrichtsalltag } \\ 7 & 161-195 & \text { Wissensvermittlung und Konfliktlösungsverhalten } \\ 8 & 200-225 & \text { Rollenverhalten und Selbstkonzept einer guten Lehrperson }\end{array}$

Bereits hier ist erkennbar, was sich in der formulierenden Interpretation bestätigt, dass es nämlich zwei Hauptinhalte gibt, die von Bianca verhandelt werden: der Theorie-Praxis-Bezug und ihre persönliche Motivation. Die Passagen, die sich besonders fokussiert mit diesen Inhalten beschäftigen, wurden somit auch für die reflektierende Interpretation ausgewählt. Hinzugenommen wurde eine Passage, die sich mit den strukturellen Vorgaben im Fremdsprachenunterricht auseinandersetzt, da dieses Thema auch für Anne eine wichtige Rolle spielt (s.u.).

\section{Formulierende und reflektierende Interpretation von vier Episoden}

Transkriptauszug 1 , „dass ich zweisprachig aufgewachsen bin“

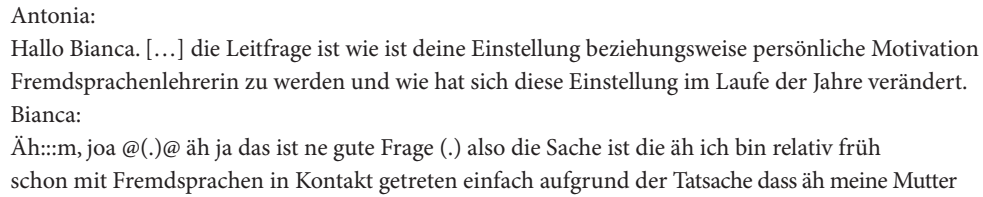


7 Spanierin ist und ich ähm zweisprachig aufgewachsen bin und ich dadurch halt einfach (.) von kleinz

8 auf äh so zu sagen immer son Zugang zu Fremdsprachen hatte (.) und ähm das hat sich dann in der

9 Schulzeit später dann auch äh (.) so fortgesetzt indem ich Französisch als erste Fremdsprache in der

10 Schule gelernt hab und ich hab da auch immer große Freude dran gehabt und es ist mir auch nie (.)

11 äh so schwer gefallen und es war relativ früh für mich auch klar dass ich äh gerne was ähm mit mit

12 Sprachen machen möchte? (2) äh::m zude::m äh::hab ich (2) dann nachdem ich mit dem Studium

13 begonnen hab also ich hab erst mal Wirtschaftsromanistik studiert und bin erst später (.) äh zum

14 Lehramt gekommen das lag einfach daran dass ich äh immer dachte ich bin nicht so:: geeignet für den

15 für den Job weil ich auch eher ungeduldig bin aber das hat @(.)@ sich im Laufe der Jahre dann (.)

16 verändert weil ich auch viel Nachhilfe gegeben hab und dann eben auch über mich hinaus gewachsen

17 bin und auch sehr viel über äh ähm mich selber dazu gelernt hab und dann hab ich (.) letztendlich

18 den Schritt gewagt und muss jetzt sagen äh ähm das war die beste Entscheidung die ich hätte treffen

19 können? (4) Ich hab da sehr große äh Freude dran ich hab auch während des Lehramtsstudiums dann äh

20 Vertretungsunterricht gemacht einige Jahre lang an einer Gesamtschule in [Ortsangabe] und (.) äh das

21 hat mich weiter in meiner Berufswahl dann (.) bestärkt (.) ähm ich konnte ja schon sehr viele

22 Erfahrungen sammeln auch fachfremd aber überwiegend halt in meinen Studienfächern Spanisch und

23 Französisch (5) u:::nd 28 äh:::m (...) ja (...)

\section{Formulierende Interpretation}

Bianca reagiert auf die Impulsfrage nach ihrer persönlichen Motivation für ihre Berufswahl mit einer biografischen Erzählung; ihre Mutter sei Spanierin, sie selbst somit „Zweisprachig aufgewachsen“. In der Schule habe sie Französisch als erste Fremdsprache gelernt und die Erfahrung gemacht, dass ihr Sprachen leicht fielen. Dies habe ihre spätere Berufswahl bereits angebahnt, allerdings habe sie mit dem Studium der Wirtschaftsromanistik begonnen, bevor sie zum Lehramt wechselte. Den Wechsel habe sie erst vollzogen, als sie durch selbst erteilten Nachhilfeunterricht entgegen ihrer ursprünglichen Vermutung die Erfahrung ihrer Eignung für den Lehrberuf gemacht habe. Der Vertretungsunterricht, den sie während ihres Studiums erteilte, habe sie in dieser Einschätzung bestärkt.

\section{Reflektierende Interpretation}

Bianca tritt nach einem kleinen Pausenfüller (Äh:::m, joa @(.)@ äh ja das ist ne gute Frage) sofort in den Modus der Erzählung ein. Sie berichtet die Etappen „Zweisprachig aufgewachsen“, „von klein auf äh so zu sagen immer son Zugang zu Fremdsprachen“ und „Französisch als erste Fremdsprache“. Das Wie der Erzählung ist in dieser Anfangsphase des Interviews noch geprägt vom Bemühen, möglichst sachlich zu bleiben und ihre Erinnerungen zu sortieren. Der Wendepunkt liegt im Wechsel des Studiengangs, den sie mit der Selbsterfahrung 
während des Nachhilfeunterrichts begründet. Sie hält sich für „eher ungeduldig“, doch sei sie „dann eben auch über mich hinaus gewachsen“. Die Erzählung strebt nun auf den Höhepunkt zu: „und dann hab ich (.) letztendlich den Schritt gewagt und muss jetzt sagen äh ähm das war die beste Entscheidung die ich hätte treffen können“. Die Entscheidung erscheint in Biancas rückblickender Erzählung wie ein großes persönliches Abenteuer, eine Grenzerfahrung, die sie erfolgreich bewältigte. Tatsächlich gehört die Berufswahl neben der Partnerwahl in dieser Lebensphase zu den Schlüsselentscheidungen eines Menschen, die Formulierungen „letztendlich den Schritt gewagt“ und „die beste Entscheidung die ich hätte treffen können“ könnten für beides verwendet werden. Diese sehr persönliche Färbung verrät, dass sich hinter Biancas Sachlichkeit Temperament und Emotionen verbergen. Entscheidend ist an dieser Stelle der frühe Aufbau einer positiven Grundrahmung des Lehrerberufs, in der die Berufswahl voll bejaht wird.

\section{Transkriptauszug 2, „weil die unglaublich motiviert sind“}

@Es ist einfach wichtig@ die Sprachen zu beherrschen aber eben auch das ganze drum herum ne? Und äh::m joa (2)@(.)@ Di::e Erfahrung die ich äh im Unterricht gemacht hab mit den mit den Sprachen beziehungsweise (3) man hat ja immer son bisschen die Befürchtung, gerade wenn man sagt äh gerade wenn man Französisch unterrichtet äh ist es ja immer son bisschen die Schwierigkeit dass äh dass die Schülerinnen und Schüler auf Anhieb ä::h die Angst haben dass sie dass die gegebenenfalls überfordert sein könnten wegen der Aussprache oder wegen der Grammatik weil (.) der O-Ton äh der Gesellschaft genau aber auch ä::h genau immer derjenige ist dass das Französisch eine Sprache ist die unglaublich schwer zu lernen ist gerade auch im Vergleich zum Spanischen (2) ähm (3) das ist halt so die Befürchtung die die Schüler (.) mitbringen in den Unterricht ich hab aber auch äh (2) gemerkt dass ä::h sowohl im Französischen aber halt auch immer im Spanischen was äh für viele vermeintlich die leichtere Sprache ist (.) ä::h ähm (3) dass äh solange man den Unterricht oder oder auch die Unterrichtsinhalte so (.) auf die auf die Welt der Schü::ler anpasst äh äh dass die da n Bezug zu sich herstellen können (2) ist das äh besteht diese Schwierigkeit di:::e mit der viele sich am Anfang konfrontiert sehen eigentlich eher im Hintergrund und es es geht letztendlich darum (.) wenn man ähm (.) die die Unterrichtsinhalte so verpackt dass die Schüler für sich immer wieder n Bezug zu ihrem eigenen Leben und zu ihren eigenen Interessen herstellen können dann ist (.) äh eigentlich die die Grammatik hmmm eher nebensächlich und dan::n lernen die Schüler ohne es vielleicht (2) bewusst ähm (3) zu merken und dann haben die da auch äh sehr viel Freude dran einfach äh (.) weil die unglaublich motiviert sind dadurch dass die dann erzählen können was sie mit ihrem Freunden machen oder oder was ihre Haustiere sind oder sie von ihrer Familie sprechen können und solche Dinge das ist einfach ähm (.) sehr schön zu sehen wie die Kinder dann motiviert sind und dann auch wirklich ä:::h (2) aufblühen und ähm und das dann alles in der Fremdsprache (2) umzusetzen das ist auf jeden Fall se::hr (2) ä::h (4) genau also sehr schön zu sehen dass das 
48 funktioniert, obwohl man vielleicht auf Anhieb denkt naja:: die zweite Fremdsprache ist immer son

49 bisschen kniffliger (.) wenn man vorher schon Probleme mit Englisch hatte aber es kommt wie gesagt

50 (.) also die Erfahrung hab ich zumindest gemacht immer darauf an wie man den Unterricht (.) aufbaut

51 und ähm (4) joa

Formulierende Interpretation

Bianca thematisiert zunächst ihre Erfahrung als beginnende Französischlehrerin. Sie äußert ihre Angst, in den Unterricht gegangen zu sein und dabei die vermuteten Schwierigkeiten der Schülerinnen und Schüler mit dem Französischen im Vergleich zu vermeintlich leichteren Sprachen nicht auffangen zu können. Diese Sorge sei jedoch sehr rasch der Erfahrung gewichen, dass diese Schwierigkeiten verblassten, wenn es ihr gelänge, die Unterrichtsinhalte an die Lebenswelt und die Interessen der Lernenden anzupassen. Die Möglichkeit, in einer anderen Sprache etwas über sich selbst zu sagen, habe die Lernenden sehr motiviert. Diese Motivation bei den Lernenden habe dann auch ihre Motivation beflügelt.

Reflektierende Interpretation

Bianca reagiert hier nicht auf eine Frage, sondern initiiert sozusagen ohne Punkt und Komma selbst das Thema Lehrermotivation durch Schülermotivation. Die Erzählung wechselt nun in eine Argumentation. Bianca stellt das Argument in den Raum, das ihrer Aussage nach auf eigenen Erfahrungen beruht, Schülerinnen und Schüler würden „auf Anhieb ä::h die Angst haben dass sie dass die gegebenenfalls überfordert sein könnten wegen der Aussprache oder wegen der Grammatik". Sie bearbeitet nun über mehrere Elaborationen hinweg dieses vermeintliche Klischee, das sie offensichtlich belastete. Zu den Elaborationen gehört die Autorität der öffentlichen Meinung („O-Ton der Gesellschaft"), der Vergleich mit dem Spanischen und die Behauptung, dass die Schüler diese „Befürchtung (.) mitbringen in den Unterricht". Sie stellt diesem negativen Vergleichshorizont der Umgebung einen positiven Gegenhorizont entgegen: Wenn man Unterrichtsinhalte „auf die Welt der Schü::ler anpasst äh äh dass die da n Bezug zu sich herstellen können“. Die Motivation zum Lernen einer Sprache steht demzufolge nicht in Bezug zum vermeintlichen Schwierigkeitsgrad dieser Sprache, sondern resultiert aus der Gelegenheit, in dieser Sprache etwas über sich selbst und seine Lebenswelt sagen zu können.

Bianca verwendet in diesem Zusammenhang auch die Metapher des „Verpackens" von Unterrichtsinhalten. Grammatik könne von Unterrichtenden so "verpackt“ werden, dass Lernende gar nicht „bewusst merken“, dass und wie sie sie gelernt haben, weil sie z.B. über ihre Haustiere und ihre Familien sprechen, d.h. über Themen, die für sie relevant sind. 
Diese längere Elaboration mit Beispielen mündet in das Thema der Freude und der Motivation: „das ist einfach ähm (.) sehr schön zu sehen wie die Kinder dann motiviert sind und dann auch wirklich ä:::h (2) aufblühen und ähm und das dann alles in der Fremdsprache (2) umzusetzen das ist auf jeden Fall se::hr (2) ä::h (4) genau also sehr schön zu sehen dass das funktioniert“. Die Wiederholung von „schön zu sehen“ signalisiert einerseits die Evidenz des Gegenhorizonts, andererseits aber auch Biancas eigene Freude bei dieser Erfahrung. Bianca orientiert sich an einer vollständigen und positiven Identifikation mit ihrer einst getroffenen Berufswahl. Diese positive Identifikation erlaube es ihr, auch eine vermeintliche Hürde im Berufsalltag mit Leichtigkeit zu nehmen.

\section{Transkriptauszu 3, „nie als Fessel empfunden“}

61 Antonia:

62 Hast du vielleicht vorher schon irgendwie darüber nachgedacht, inwiefern dich Lehrpläne

63 oder das Kerncurriculum oder Bildungsstandards irgendwie (.) einschränken könnten? Oder welche

64 Erfahrungen hast du da gemacht?

65 Bianca:

66 Also ich hab die Erfahrung gemacht natürlich gibt es äh äh Vorgaben und die die vgibt es auch

67 nicht ohne Grund und das find ich auch wichtig ähm ich hab das aber nie so als als als Fessel oder als

68 Einschränkung empfunden wei::l äh::m (.) man trotzdem innerhalb dieser dieser Vorgaben die

69 teilweise natürlich auch sehr konkret und sehr strickt sind äh trotzdem noch sehr viel Spielraum hat

70 (.) die Inhalte so wie sie vorgegeben sind ähmm eben auf die entsprechenden Lerngruppen und den

71 Unterricht anzupassen (.) weshalb ich da nie in irgendnen Konflikt geraten bin äh::m zu sagen ohje (2) ich hab jetzt hier die und die Vorgabe lässt sich aber so eigentlich gar nich umsetzen weils halt immer darauf ankommt äh wie man eben den entsprechenden Unterrichtsinhalt auf die auf die Gruppe anpasst zu de::m ist dass auch so dass ä::h das es zur Zeit sehr viele (.) auch gute Lehrwerke gibt, (.) die den Unterricht natürlich begleiten gerade im Anfängerunterricht ist es es ist ja sehr lehrwerkgestützt ähm (.) und die sind natürlich auch von den von den Inhalten und ähm und den Themen soweit an di::e an das Kerncurriculum angepasst dass es ja eigentlich (.) äh wenig Schwierigkeiten gibt sich nicht daran zu halten aber man hat wie gesagt trotzdem noch (.) innerhalb der Vorgabe sehr viele Freiheiten und Möglichkeiten das äh auf die entsprechende Lerngruppe (..) umzusetzen, weshalb ich persönlich jetzt nicht das Gefühl hatte dass ich dass ich da irgendwi::e ä::h ne ne Fessel (2) also ich hab das nie als Fessel empfunden ä::h eher auch als Hilfe, gerade also als 82 Berufseinsteiger (.) wo man (.) teilweise vor ner äh vor nem riesen Berg an an an Materialien und 83 Information steht die man irgendwie weitergeben muss äh äh $\mathrm{k}$ in jedem Fall ne (.) ne gute Hilfe (.) äh sich selbst und den Unterricht (.) äh zu strukturieren, (2) und wie gesagt man muss halt schauen was man daraus macht und ähm wie man es umsetzt aber äh bisher hat es immer (.) relativ reibungslos 
Formulierende Interpretation

Die Interviewerin versucht, Bianca durch die Frage nach eventuell als Einschränkung empfundenen offiziellen Vorgaben auf mögliche Konflikte in ihrer beruflichen Erfahrung zu lenken. Bianca äußert daraufhin, dass diese Vorgaben hilfreich aber gleichzeitig so allgemein seien, dass es genügend Spielräume für individuelle Anpassungen gäbe. Gleichzeitig äußert sie die Ansicht, dass auch die Lehrwerke, die ihrerseits den Vorgaben angepasst seien, besonders im Anfängerunterricht eine unterstützende Funktion für die Lehrpersonen ausübten. Die Situation des Berufsanfängers sei dadurch geprägt, dass er angesichts der Materialfülle eine vorgegebene Struktur benötige, die er in den Lehrplänen und Lehrwerken durchaus vorfände.

\section{Reflektierende Interpretation}

Die Interviewerin initiiert nach Biancas euphorischer Schilderung ihres Berufserlebens ein Thema, das Bianca vielleicht herausfordern könnte, und bittet sie, auch einmal von einer Konflikterfahrung zu berichten. Bianca reagiert darauf mit einer Argumentation, die zwar im Modus einer Erzählung beginnt („Also ich hab die Erfahrung gemacht"), dann aber sofort eine logische Struktur aufbaut: Vorgaben gäbe es „nicht ohne Grund“, und sie hätten das Merkmal „natürlich sehr konkret und sehr strikt“ zu sein sowie „sehr viel Spielraum“ zu lassen. Bianca äußert damit die Orientierung eines grundsätzlichen Vertrauens in die Schulverwaltung, die die Vorgaben macht. Da es - wie schon in der ersten Passage ausgeführt - darauf ankomme, die Unterrichtsinhalte an die Lerngruppe anzupassen, entstünden auch keine Konflikte. Bianca schlägt nun noch den erweiternden Bogen zum Subthema „Lehrwerke“, das ebenfalls mit einer grundsätzlich positiven Orientierung verbunden ist: Lehrwerke unterstützen laut Bianca Unterrichtende, sie „begleiten“ sie, was als besonders angenehme und jedenfalls nicht gängelnde Form der Unterstützung interpretiert werden kann. Bianca fügt mehrfach das Adverb „natürlich“ hinzu, sie attribuiert Vorgaben und Lehrwerken somit eine nicht hinterfragbare Rolle, eine positive Selbstverständlichkeit, wodurch Unterricht aus ihrer Sicht den Charakter einer Naturgesetzlichkeit erhält.

Die Argumentationskette wird abgeschlossen mit dem Hinweis auf die Situation des Berufsanfängers, der „vor einem Berg an Materialien und Informationen steht, die man irgendwie weitergeben muss". Erneut betont Bianca, dass Vorgaben und Materialien eine Hilfe darstellen und keinesfalls „eine Fessel“. 
Bianca benutzt die Fessel-Metapher zweimal, stets verneinend („nie als Fessel“) und betont damit ihre völlige Kongruenz mit der positiven Rahmung des Unterrichts, die sie bereits in den Vorgängerpassagen konsequent aufgebaut und verfolgt hat. In dieser Grundrahmung sind Probleme Herausforderungen, die mit Hilfe der vorhandenen Unterstützungsinstrumente gut bewältigt werden können. Ziel ist, am Berufsanfang, „,sich selbst und den Unterricht (.) äh zu strukturieren". Mit anderen Worten: Gelingender Unterricht setzt in erster Linie Strukturiertheit voraus. Die konkludierende Synthese „bisher hat es immer (.) relativ reibungslos funktioniert" impliziert ein hohes Maß an Sicherheit. Es hat nicht nur funktioniert, sondern sogar relativ reibungslos.

\section{Transkriptauszug 4, ,letztendlich ist aber die Realität im Schulalltag ne ganz an- dere“}

85 Antonia:

86 Hast du bei den Erfahrungen die du beim Unterrichten schon gemacht hast (2) gemerkt dass

87 sich irgendwie (.) Probleme ergeben die man vorher im Studium vielleicht noch nicht so gedacht hat

88 oder hat es irgendwie Situationen gegeben bei denen du gedacht hast dass ähm- (3)

89 Bianca:

90 Also was ich äh was ich auf jeden Fall gemerkt hab selber in den Unterrichtserfahrungen auch

91 rückblickend dann auf das Studium (.) wo man auch viele ähm Seminare natürlich äh gemacht hat

92 auch zum Thema Grammatikvermittlung und solche Sachen also da (2) muss ich leider sagen die

93 Sachen an der Uni die waren alle unglaublich interessant und ich bin gerne dahin gegangen

94 letztendlich ist aber die die Realität im Schulalltag ne ganz andere also (.) es gibt (.) sicherlich sehr sehr viele äh äh Sachen die dann ein gutes Polster geschaffen haben aber ähm letztendlich wenn man dann konkret in der Unterrichtssituation steht und und sich überlegt wie muss ich jetzt oder wie kann ich am (.) am besten und effektivsten diese und diese Grammatik meinetwegen an den an den Schüler bringen und ihm auch so vermitteln dass er sie auch anwenden kann aber auch immer nen Kontext hat (.) indem er sie sinnvoll? für sich auch anwenden kann sie dadurch besser zu verinnerlichen (.) @ da muss ich leider sagen @ ist es äh eher (.) äh learning by doing und direkt an der Front in der Schule weil da (2) hätt ich mir vielleicht im Nachhinein gewünscht dass man an der Uni vielleicht die ein oder andern Methoden vielleicht nochmal nen bisschen genauer betrachtet äh beziehungsweise nochma die ein oder andere Lernstrategi::e äh genauer durchgeht aber (3) wobei ich aber auch sagen muss das kann man ja auch so (.) global gar nicht (.) äh gar nicht sagen weil jede Lerngruppe anders ist und und und die gleichen Inhalte müssen in der einen Gruppe so vermittelt werden und in der anderen Gruppe so es ist halt natürlich (.) wenn man mit Menschen zusammenarbeitet kann man das nicht immer gleich aufzie::hn (.) deshalb äh (3) seh ich äh meinen Job auch einfach als äh äh selber für mich immer noch als Lernprozess äh (.) ich lerne von den Schülern mindestens genauso viel wie die von mir wenn nicht sogar noch meh::r einfach auch äh die (.) über die Art und Weise wie mans vermittelt und welche Methoden man anwendet (.) viele Gruppen möchten einfach nach wie vor frontal unterrichtet werden, für andere Gruppen ist das gar nix (.) es ist (.) immer ganz unterschiedlich aber aber so jetzt (.) wenn ich wirklich zurückblicke (.) muss ich sagen dass was ich äh was ich jetzt im Laufe der Jahre an Erfahrung gemacht hab::e; und was mich zu der Lehrerin gemacht hat die ich heute bin (.) waren jetzt weniger di::e theoriegeleiteten Seminare an der Uni als wirklich die die Unterrichtspraxis selber ne? weil das ist (3) wie gesagt (2) 
116 Klar es ist interessant zu gucken welche Motivation haben Fremdsprachenlerner und äh (.) wie können

117 die sich über den Unterricht hinaus noch (.) äh weiterbilden und so (2) das die andere Sache ist halt die

118 (.) dass man jetz::t äh bei nem dreizehnjährigen Schüler aus der siebten Klasse nicht per se

119 unterstellen kann dass er über den Unterricht den er in der Schule hinaus genießt @ zu Hause noch @

120 Lektüren in der Fremdsprache liest oder oder äh oder Filme anschaut um einfach sprachlich sich

121 weiter- äh zu bilden das ist einfach (.) das passiert sicherlich aber finde ich äh eher (.) utopisch und

122 halt so über die Dinge über die man in der Uni auch gesprochen hat das ist so unglaublich interessant

123 und so für mich (.) persönlich war das auch ne Bereicherung, aber (.) das lässt sich halt alles nicht

124 so:::: optimal auf den auf den eigen eigenen wirklichen Unterrichtsalltag äh zuschneiden

125 beziehungsweise anwenden (.) weshalb (2) es letztendlich äh wirklich so ist wie ich ja eben schon

126 gesagt hab ähm man muss einfach schauen, was hat man für (.) für Schüler vor sich sitzen wie passt

127 das menschlich äh weil ich auch die Erfahrung gemacht hab das wenn das (.) wenn das

128 Zwischenmenschliche zwischen Lehrperson und den und den Lernenden nicht (.) stimmt dann ist es

129 auch (.) wesentlich schwierig::er die Inhalte angemessen zu vermitteln als wenn jetzt (.) die die

130 Chemie total gut ist von Anfang an und man einfach auch ne ne Gruppe ist (.) in der jede::r äh sich

131 auch eingeladen fühlen kann seinen Beitrag zu leisten und wo man auch angemessen mit Fe::hlern

132 umgeht weil das auch sind Dinge die (.) die häufig passieren und ähm (.) wie gesagt also da muss ich

133 leider sagen hab ich aus der Uni nicht so viel (2) mitgenommen? ä:::::hm was jetzt speziell die

134 Unterrichtspraxis betrifft (.) ja aber son:::st (.) joa also man muss halt einfach es gibt halt viele

135 Faktoren die die die guten Unterricht äh ausmachen beziehungsweise di::e (.) die den Unterricht am

136 Laufen halten und überhaupt Unterricht möglich machen und das ähm:::m das sind halt Dinge die

137 kann man aber auch vorher nicht antizipieren weil viele Sachen (.) eben wie (2) diese

138 zwischenmenschliche Beziehung das kann man nich üben (3) Also entweder man man kriegt das

139 irgendwie hin und das passt menschlich oder halt eben nich ne? Weil man muss sich halt immer

140 bewusst machen wenn man als Lehrer (.) in die Lerngruppe rein geht da sitzen::n zwischen 20 und 25

141 unterschiedliche Menschen, (.) die genauso ihre ihre Macken und Launen haben wie man selber auch

142 als Lehrer und da muss man halt schauen das man da ne (.) ne professionelle Basis findet auf der man

143 (.) aufbauen kann und mit der man dann arbeiten kann

\section{Formulierende Interpretation}

Die Interviewerin lenkt den Blick erneut auf mögliche Probleme und thematisiert dabei das Wissen aus dem Studium. Bianca erwidert, dass sie im Studium zwar für sie persönlich sehr spannende fachdidaktische und bildungswissenschaftliche Veranstaltungen besucht und eine breite Wissensbasis erworben habe, in der Schulpraxis auf dieses Wissen jedoch nicht habe zurückgreifen können. Der Grund liege in der notwendigen Anpassung an die jeweiligen Lerngruppen und an die stets neuen Lernsituationen. Sie befinde sich in einem permanenten wechselseitigen Lernverhältnis, die Schüler lernten von ihr und sie von ihnen. Den entscheidenden Faktor für gelingenden Unterricht, nämlich mit einer großen Anzahl Individuen auf der zwischenmenschlichen Ebene klarzukommen und eine "professionelle Basis" aufzubauen, habe sie im Studium und in der fachdidaktischen Theorie nicht gelernt, sondern erst im Berufsalltag. 
Reflektierende Interpretation

Die Interviewerin stößt das Thema „Vergleich Unterrichtspraxis - Studium“ an. Bianca greift dieses Thema bereitwillig auf und proponiert sofort argumentierend den Gegenhorizont, Schulalltag und Studium seien völlig verschiedene Realitäten: „letztendlich ist aber die die Realität im Schulalltag ne ganz andere“. Diesen Gegenhorizont, der im Folgenden auf vielfältige Weise elaboriert wird, leitet Bianca durch eine abmildernde Vorrede ein, in der sie rhetorisch positive Wertungen für die universitären Seminare äußert („die Sachen an der Uni die waren alle unglaublich interessant und ich bin gerne dahin gegangen), diese jedoch durch „muss ich leider sagen“ und „aber" entwertet. Dieses rhetorische Mittel der Auf- und Abwertung kommt nach der Formulierung des Gegenhorizonts noch einmal zur Anwendung, indem Bianca „Sachen die dann ein gutes Polster geschaffen haben“ erwähnt und das Beispiel der Grammatik elaboriert. Bereits hier lässt sie die ihrer Auffassung nach geringe Tauglichkeit allgemeiner Theorien für den Unterrichtsalltag erkennen, denn das Beispiel mündet in die Aussage „@ da muss ich leider sagen @ ist es äh eher (.) äh learning by doing und direkt an der Front in der Schule“. Die beiden Fokussierungsmetaphern bringen Biancas Orientierung zum Ausdruck, wonach sich pädagogisches und fachdidaktisches theoretisches Wissen seinem Wesen nach vom Praxiswissen unterscheidet. Dieses Praxiswissen hat im Gegensatz zum theoretischen Wissen die Merkmale, erstens aus praktischem Handeln generiert zu sein („learning by doing") und nicht aus Belehrung, und zweitens den Abdruck der Interaktion mit den Schülern zu tragen („direkt an der Front“). Die martialisch anmutende Fokussierungsmetapher „direkt an der Front“ betont rhetorisch den Gegensatz Front versus grüner Tisch. Wissen in Hochschulseminaren ist sozusagen am grünen Tisch generiertes Wissen, Wissen aus praktischem Lehrerhandeln ist Frontwissen, im Einsatz geprüftes Wissen.

Bianca elaboriert nun diese Orientierung an weiteren Beispielen. So führt sie Methoden und Lernstrategien an, die ihr in ihrer fachdidaktischen Ausbildung im Studium zu kurz gekommen seien, relativiert dann aber selbst diese Forderung mit dem Hinweis auf die Abhängigkeit der Methoden von der jeweiligen Lerngruppe. Ein weiterer starker Gegenhorizont zum fachdidaktischen theoretischen Wissen kommt in dem Beispiel der Motivation zum Ausdruck. Hier führt sie eine Theorie an, die angeblich suggeriere, Lernende auch über den Unterricht hinaus durch Filme und Lektüren für die Fremdsprache anzuregen. Diese 
Vorstellung bezeichnet Bianca als „utopisch“. Fachdidaktische Theorie erscheint mithin als teilweise wirklichkeitsfern. Sie wiederholt sodann suggestiv den Topos der hochinteressanten Theorien an der Universität, die jedoch, um brauchbar zu sein, erst in der Praxis transformiert werden müssten. Auch das Argument der herausragenden Bedeutung der Lehrer-Schüler-Beziehung („zwischenmenschliche Beziehung") taucht wieder auf, ein Aspekt, der in der hochschuldidaktischen Ausbildung nur indirekt eine Rolle spielt. Bianca formuliert daraus einen Vorwurf an die Adresse der Hochschule: „also da muss ich leider sagen hab ich aus der Uni nicht so viel (2) mitgenommen“, um gleich darauf diesen Vorwurf mit dem Hinweis zu entkräften, dass die Lehrer-Schüler-Beziehung eben nicht geübt werden könne, sondern erst in der Praxis vorläge. Die Fähigkeit zum Aufbau einer guten Lehrer-Schüler-Beziehung sei wiederum ein Persönlichkeitsmerkmal und damit nicht studierbar: „Also entweder man man kriegt das irgendwie hin und das passt menschlich oder halt eben nich ne?". Bianca bezeichnet diese Grundlage für gelingenden Unterricht im Modus einer synthetisierenden Konklusion als „professionelle Basis“.

Es zeigt sich an diesen Transkriptausschnitten, dass Bianca eine implizite Normalitätserwartung vieler Studierender teilt, nach der pädagogische und fachdidaktische Seminare an der Universität wenig Relevanz für den Unterrichtsalltag besäßen. Bianca ist berufsbiografisch noch relativ nahe bei den Studierenden und nimmt sozusagen die Rolle einer Anführerin in der peer group ein, da sie ihnen Referendariat und ein Jahr Schulerfahrung voraus hat. Diese Rolle wird zu einer Art Attitüde, die sich so zusammenfassen lässt: Bianca ist eine entscheidende Erfahrung weiter, sie kennt jetzt die Realität. Das Wie ihrer Attitüde verrät jedoch, dass sie pädagogische und fachdidaktische Theorie durchaus verinnerlicht hat, denn sie artikuliert sich auf einem begrifflichen Niveau, das didaktische Kenntnisse voraussetzt.

Wie auch Anne stellt Bianca die persönliche Beziehung zu den Schülerinnen und Schülern in den Mittelpunkt. Dieses Thema sorgt zusammen mit dem Erleben der Anwendungsbegeisterung bei den Lernenden für die eigentliche Motivation beim Lehrerberuf. 


\subsubsection{Interview mit Anne}

Die thematische Grobgliederung des Interviews mit Anne weist zwölf Themen auf. An einer Stelle, beim Thema „Begeisterung für das Fach“ (hervorgehoben), greift Anne ein Thema ein zweites Mal auf, um es zu variieren und weiter zu elaborieren.

Tabelle 12: Narratives Interview „Anne“, thematische Gliederung

$\begin{array}{lcl}\text { Abschnitt } & \text { Zeilen } & \text { Thema } \\ 1 & 001-044 & \text { Annes Studien-, Berufs- und Fachwahl } \\ 2 & 049-070 & \text { Vergleich Fremdsprachenunterricht mit Kunstunterricht } \\ 3 & 074-098 & \text { Korrekturaufwand und Herausforderung an ihre Geduld } \\ 4 & 099-104 & \text { Positive Effekte } \\ 5 & 109-121 & \text { Authentisches Material } \\ 7 & 124-140 & \text { Die menschliche Beziehung: das Wichtigste im Lehrerberuf } \\ 8 & 141-150 & \text { Begeisterung für das Fach } \\ & 226-266 & \text { Begeisterung für das Fach } \\ 9 & 152-188 & \text { Fachliche Kompetenzen } \\ 10 & 192-212 & \text { Sprechen im FSU und Gruppengröße } \\ 11 & 270-287 & \text { Bewertungsproblematik } \\ 12 & 290-341 & \text { Aufrechterhaltung der Motivation }\end{array}$

Für die Auswahl der Passagen, die in Form der reflektierenden Interpretation näher untersucht werden sollten, waren auch die vom emotionslos-nüchternen Grundton (Transkriptausszug 1) abweichenden emotionalen Momente ausschlaggebend: das emotionale Thema „Korrekturaufwand“ (Transkriptausszug 2) sowie die ebenfalls emotionale narrative Passage zur Chemie-Praktikantin im Thema „Aufrechterhaltung der Motivation“ (Transkriptauszug 3). Zur Erinnerung seien noch einmal die Impulsfragen des Interviews genannt, nämlich warum sich Anne für diesen Beruf entschieden hatte und welche Bilanz sie nach einem Jahr in der Schule zog. 


\section{Transkriptauszug 1, „dieses in Anführungszeichen Schulspanisch“}

26 Anne Also erstmal bin ich vom eigenen Interesse ausgegangen und da haben mich immer eher musische, künstlerische und ähm sprachliche Fächer begeistert (.) und nicht so die Naturwissenschaften. Und ähm es ist natürlich was anderes das dann in der Schule zu unterrichten, weil weil man nen völlig anderen Bezug zur Sprache nochmal kriegt als wenn man jetzt die Sprache für sich aus äh Interesse lernt. Weil wenn man im Land ist und so, dann lernt man ganz anderes Spanisch, Französisch

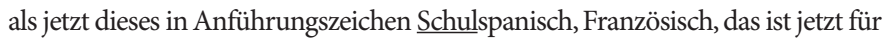
mich nochmal was völlig neues und also als ich am Anfang mich davon () äh dafür entschieden habe Sprachen in der Schule zu unterrichten war mir das nicht so klar, dass das so anders ist. Und äh:::m aber ja ich hab mich doch dafür entschieden (.) erstens eben wie gesagt vom Interesse her und zweitens weil ich das auch wichtig finde, dass die Kinder so viele Sprachen wie möglich lernen und ja (10) und die Sprache geht leider immer mehr verloren, weil ja alle Englisch äh können oder können müssen, was ja auch gut ist. Aber so die romanischen Sprachen (.) sind jetzt in den Schulen immer nicht ganz so stark vertreten. Spanisch ist ja jetzt immer mehr gekommen; und deswegen bin ich immer damit beschäftigt ja Französisch zu erhalten und Spanisch neu einzubringen und das finde ich wichtig, dass die auch noch romanische Sprachen lernen können und dass das Angebot in der Schule gibt Und gerade weil ja auch ähm (.) Lateinamerika und ähm ja spanischsprachige Länder auch ähm immer wichtiger werden.

Florian Findest du das

Anne

$L_{\text {ja also nicht für jeden aber }}$

Formulierende Interpretation

Anne formuliert ihre Berufswahlmotivation. Sie führt ihr Interesse an musischen, künstlerischen und sprachlichen Fächern an, den besonderen Bezug, den man durch das Unterrichten zu den Sprachen erhält sowie den Wunsch dazu beizutragen, dass Kinder neben Englisch möglichst viele weitere Sprachen lernen.

\section{Reflektierende Interpretation}

Der Wechsel der Analyseeinstellung vom Was zum Wie zeigt zunächst, dass Anne, wie auch Bianca, generell sehr bedacht spricht, sehr darauf achtet, nichts Vorschnelles zu äußern. Sobald sie sich etwas aus der Deckung wagt, sichert sie sich ab.

Anne beginnt das Thema der Berufswahlmotivation im Modus der Erzählung („Also erstmal bin ich vom eigenen Interesse ausgegangen“), wechselt jedoch rasch in die Argumentation („es ist natürlich was anderes das dann in der Schule zu unterrichten, weil [...] "), da sie nun einen ersten Konflikt bearbeiten muss. Dieser besteht darin, dass die Sprachaufenthalte „aus Interesse“ einen „Völlig anderen Bezug“ zu der Zielsprache herstellen als der Unterricht. Das im 
Unterricht gesprochene Spanisch qualifiziert sie als „dieses in Anführungszeichen Schulspanisch“. Hierin liegt eine gewisse Abwertung: Schulspanisch wird nicht nur nicht mit Interesse gelernt, es ist darüber hinaus auch unauthentisch („man ist nicht im Land“). Jetzt wechselt Anne wieder in die Erzählung zurück: „also als ich am Anfang mich davon () äh dafür entschieden habe Sprachen in der Schule zu unterrichten war mir das nicht so klar, dass das so anders ist", das heißt, sie erachtet den argumentierenden Einschub zum „Schulspanisch“ als eine für die Kohärenz der Erzählung notwendige Erklärung. Dieser Einschub macht jedoch weitere Erklärungen notwendig, denn Anne hat sich für einen pädagogischen Beruf entschieden und nicht etwa für eine Dolmetscher- und Übersetzerausbildung. Objektiv betrachtet wäre der Einschub keinesfalls zwingend gewesen, die Erzählung wirkte ohne diesen Einschub von außen betrachtet nicht inkohärent. Es ist vielmehr der von Anne selbst empfundene Kontrast von Unterrichtspraxis bzw. „Schulspanisch“ und authentischem Spanisch, der sie zur Argumentation veranlasst. Durch diese Rechtfertigung einer Normalitätsabweichung verwickelt sie sich allerdings gleich wieder in einen Widerspruch, denn bei der nun folgenden Nennung der Gründe für ihre Studien- und Berufswahl beginnt sie mit „hab mich doch dafür entschieden (.) erstens eben wie gesagt vom Interesse her", obwohl klar war, dass „Interesse“ anders belegt war. Anne nennt nun weitere Gründe für ihre Berufswahl: „weil ich das auch wichtig finde, dass die Kinder so viele Sprachen wie möglich lernen und ja (10) und die Sprache geht leider immer mehr verloren, weil ja alle Englisch äh können, oder können müssen, was ja auch gut ist“. Dieses sprachenpolitische Credo mutet nach den Ausführungen zum „Schulspanisch“ etwas konstruiert an, sie scheint bemüht, jetzt eine überzeugende Begründung zu nennen. Neben dem übermächtigen Englisch, das die Existenz der romanischen Sprachen bedrohe, kämpft Anne angeblich auch für den Erhalt des Französischen gegen das aufholende Spanisch. Schließlich bringt sie sogar noch die Wichtigkeit Lateinamerikas und der spanischsprechenden Länder ins Spiel, alles Argumente auf einer politischen Ebene. In dieser Passage sticht gegen die Verstandesargumente der Konflikt auf der Ebene des persönlichen Spracherlebens hervor, der durch die Fokussierungsmetapher vom ,in Anführungszeichen Schulspanisch“, das ihr vor dem Unterrichten „nicht so klar war“, symbolisiert wird.

Transkriptauszug 2, „Korrekturen“

Nach einer Weile versucht es Florian auf direktem Wege, sie aus der Reserve zu locken: „Also, dass du mal so Momente gehabt hast, wo du so gesagt hast jetzt hab ich keinen Bock mehr auf die Sprache?" (73 f.) 
Ja, also bei [räuspern] also, also negativ ist ist sind für mich eindeutig diese Korrekturen. Das kostet so viel Zeit und da hatte ich vorher in dem Ausmaße nicht mit gerechnet (3) und ähm das ist einfach nicht so meins @, da immer mit dem roten Stift alles anzukreiden äh:::m (.) ja man hat halt Vorgaben dass man die Klausuren schreiben muss, aber (.) für mich ist das immer ein bisschen anstrengend, weil ich vom Grundsatz nen anderen Ansatz hab ä:::h zu Unterrichten. Ich würde zum Beispiel wenn ich mit der ersten Fremdsprache anfange am Anfang überhaupt gar nicht auf Schriftsprache eingehen und noch keine Klausuren schreiben, sondern erst mal vom Sprechen aus (.) weil man ja selber so im Studium oder auch wenn man im Land gelebt hat die Erfahrung gemacht hat, oder ich zu mindestens, dass äh man ne Sprache übers Sprechen und Imitieren lernt und deswegen finde ich das oft kontraproduktiv und deswegen fällts mir jetzt persönlich immer schwer da (.) permanent Klausuren zu haben es kostet mich auch irre viel Zeit, ähm aber das ist jetzt eigentlich das Einzige was ich jetzt (.) als Unterrichtsfach Sprache jetzt schwierig finde.

Formulierende Interpretation

Anne nennt die Korrekturen als einen konkreten Kritikpunkt an ihrer Berufspraxis. Klausuren entsprächen weder ihrem pädagogischen noch ihrem didaktischen Selbstverständnis. Aus pädagogischer Sicht würde sie auf die Fehlerbewertung verzichten, und aus didaktischer Sicht würde sie im Anfangsunterricht der Mündlichkeit gegenüber der Schriftlichkeit den Vorzug geben. Dies entspräche auch dem natürlichen Fremdsprachenerwerb im Zielland. Anne problematisiert auch den hohen Korrekturaufwand.

\section{Reflektierende Interpretation}

Es ist einer der seltenen Momente, in denen sich Anne rundheraus zu einem Negativurteil durchringt. Sie bleibt jetzt ganz in der Argumentation, in der sie ihre Proposition „also, also negativ ist ist für mich eindeutig diese Korrekturen“ elaboriert und über mehrere Stationen hinweg konsequent aufbaut:

1. „Es kostet so viel Zeit“

2. Damit hatte sie „vorher in dem Ausmaße nicht gerechnet“

3. „Ist einfach nicht so meins @, da immer mit dem roten Stift alles anzukreiden“

4. „man hat halt Vorgaben“

5. „weil ich vom Grundsatz her nen anderen Ansatz hab“

6. „kontraproduktiv“

Diese Argumentation wirkt nicht konstruiert, sondern sehr spontan. Interpretatorisch ist die Metapher „mit dem roten Stift alles anzukreiden“ interessant, weil sie auf eine fundamentale Normabweichung Annes hinweist. Schließlich gehört das Bewerten und Benoten von Anfang an zum Berufsalltag einer Gymnasiallehrerin. Anne versucht auch nicht, diese Abweichung kleinzureden, sondern erhärtet sie noch: „weil ich vom Grundsatz her nen anderen Ansatz hab“. Der Dissens ist also 
grundsätzlicher Natur. Der Bezug zum Sprachenlernen in Spanien als Kontrast zum „Schulspanisch“ wurde bereits in der ersten Passage bearbeitet und bleibt virulent.

Schließlich spricht sie auch noch die institutionell-strukturelle Ebene an („man hat halt Vorgaben“), die noch mehrfach im Verlauf des Interviews eine Rolle spielen wird.

\section{Transkriptauszug 3, „das dauert halt ewig“}

88 Ähm ja ansonsten fordert es auch oft sehr viel Geduld (2) im Spanischen geht's noch weil vom

89 Deutschen her, wenn man jetzt Deutsch als Muttersprache hat nicht so::: schwierig ist am Anfang

90 weil man ja jeden Buchstaben spricht aber gerade bei den Kleinen jetzt hab ich ne sechste Klasse

91 (.) also die tun sich wirklich sehr schwer das Französische auszusprechen (.) und das dauert halt

92 ewig; da kann man das auch zehnmal vorsagen. Das dauert einfach bis die sich das merken da

93 muss man manchmal sich selber jedes Mal wieder klar machen, die haben einfach gar kein

94 Sprachgefühl und das können die aber auch noch nicht haben und dann geht's; aber das ist

95 manchmal auch etwas anstrengend.

96 @nd wenn sich dann immer so [undeutlich] da rein mogelt ähm aus dem Englischen dann

97 denkt man auch manchmal auch ach naja Transfer äh Sprache hin oder her aber [undeutlich]

98 manchmal auch anstrengend. $\mathrm{Ne}$ ?

99 Aber positiv ist eigentlich mehr. Also jetzt gerade von den Effekten die man so hat (.) die freuen

100 sich dann wenn se dann schon mal einen Text versteh:en. Oder wenn die in Spanien im Urlaub

101 waren und äh schon was verstanden haben oder ähm (5) ja Lieder über Lieder, wenn die dann

102 plötzlich merken ohhh das Lied kenn ich und ach das heißt das also (.)

103 Ja und schön ist auch wenn sich dann schon nach ein paar Wochen äh tatsächlich schon ein

104 bisschen in der Sprache unterhalten können (.)

\section{Formulierende Interpretation}

Die Passage schließt unmittelbar an die vorherige Passage „Korrekturen“ an. Anne spricht das Thema „Geduld“ an und führt dazu die Mühen des Anfangsunterrichts aus. Die Lehrkraft müsse in dieser Phase sehr viel Geduld aufbringen, bis bei den Schülern erste Strukturen automatisiert zur Verfügung stünden. Dazu kämen gelegentlich negative Transfereffekte aus dem Englischen. Anne schlägt dann aber die Brücke zu den positiven „Effekten“ des Anfangsunterrichts auf Grund der ersten Erfolgserlebnisse. Diese wiederum führten auch bei der Lehrkraft zu einem Positiverlebnis.

\section{Reflektierende Interpretation}

Anne wechselt in diesem Auszug von der Argumentation wieder in die Beschreibung. Sie beschreibt die Geduld, die beim Anfangsunterricht erforderlich ist. Die Beschreibung ist an dieser Stelle sehr detailliert (Detaillierungszwang, s. Kap. 2.4), 
was darauf schließen lässt, dass dieser Punkt für sie hohe Relevanz besitzt. Sie beschreibt den Bezug zum Deutschen als Muttersprache, ,weil man ja jeden Buchstaben spricht aber gerade bei den Kleinen jetzt hab ich ne 6. Klasse (.) also die tun sich wirklich sehr schwer das Französische auszusprechen“. Die übertreibende Formulierung „und das dauert halt ewig; da kann man das auch zehnmal vorsagen" impliziert ein recht negatives Bild aus Sicht der Lehrperson: Alles dauert sehr lange. Anne wiederholt sich nun selbst: „Das dauert einfach bis die sich das merken da muss man manchmal sich selber jedes Mal wieder klar machen", was das negative Bild weiter verstärkt. Schließlich konstatiert sie fast resigniert: „die haben einfach gar kein Sprachgefühl“. Diese apodiktisch anmutende Formulierung lässt scheinbar keine Hoffnung aufkommen, denn ohne Sprachgefühl wird das Sprachenlernen immer rudimentär bleiben. Hier klingt wieder das bereits mehrfach geäußerte Thema "Schulspanisch“ an. Anne orientiert sich an einer sehr hohen sprachlichen Norm, dem authentischen Sprachgebrauch, der noch dazu nur im Land selbst richtig gelernt werden kann. Daran gemessen kann der Fremdsprachenunterricht immer nur minderwertige Ergebnisse erbringen. Der Lernersprache wird von Anne dagegen kein besonderer Wert zugesprochen, womit sie von fachdidaktischen Positionen abweicht. Allerdings relativiert Anne ihre negative Bewertung der Lernersprache gleich wieder: „und das können die aber auch noch nicht haben und dann geht's". Dieser relativierende Habitus lässt sich durchgängig bei ihr beobachten. So bedauerte sie bereits in der ersten Sequenz die Dominanz des Englischen („weil ja alle Englisch äh können oder können müssen“), sichert sich aber sofort wieder durch eine Relativierung ab („was ja auch gut ist“). Anne ist so ehrlich, zu sagen, was sie wirklich denkt, möchte aber damit kein Risiko eingehen. Soziogenetisch könnte hier möglicherweise auf Erfahrungen aus dem Studium und besonders dem Referendariat verwiesen werden, wo persönliche Festlegungen oft in Frage gestellt, ja sanktioniert werden.

Anne elaboriert das Thema ,anstrengender Anfangsunterricht“ nun am Beispiel des Transfers aus dem Englischen weiter. Anne betont allerdings den negativen Transfer aus dem Englischen und schlägt dann die Brücke zu positiven „Effekten“ im Anfangsunterricht. Dieses Thema wird von ihr an drei Beispielen elaboriert, dem ersten Textverständnis, den Erfolgserlebnissen beim Spanienurlaub und dem Wiedererkennen und Verstehen von Liedern. Diese dreifache positive Elaboration pendelt sozusagen die negative Elaboration zum mangelnden Sprachgefühl aus, ein diskursiver Habitus, der wie erwähnt das ganze Interview durchzieht. Anne sucht immer wieder den Ausgleich, begibt sich nie vollständig in eine kritische Haltung hinein. Die Passage konkludiert sie mit der synthetisierenden Zusammenfassung: „Ja und schön ist auch wenn sich dann schon nach ein paar Wochen äh tatsächlich 
schon ein bisschen in der Sprache unterhalten können“. Dies zeigt wieder die positive Orientierung Annes am pädagogischen Erfolg. Die Sicht ist jetzt schülerzentriert, obwohl auch ihr eigenes positives Erleben mit einbezogen wird.

\section{Transkriptauszug 4, „Praktikanten oder Referendare“}

\begin{tabular}{|c|c|}
\hline & Und dann find ich es wichtig () dass mach ich auch jetzt schon () finde hier an der Schule \\
\hline 307 & ist das auch sehr gut dass man sich permanent mit Kollegen austauscht weil man dann \\
\hline 308 & einfach andere Ideen kriegt oder auch gerade Praktikanten oder Referendare da sind ne die \\
\hline 309 & haben auch immer andere Ideen oder wenn Leute aus anderen Fächern kommen und die \\
\hline & gleiche () also auch einfach die gleiche Kombi haben () die haben völlig andere Ansätze \\
\hline 311 & was beizubringen \\
\hline 312 & Zum Beispiel hatte ich jetzt in Kunst eine da habe ich () da geht's manchmal um \\
\hline 313 & Farbenlehre [räuspern] und da hatte ich eine Referendarin die hat als Zweitfach Chemie und \\
\hline 314 & Chemie war für mich immer () also völlig weit weg () konnte ich nie mit was anfangen (.) \\
\hline 315 & und ähm die hat Farbenlehre die ähm (.) war ganz anders ähm den Schülern beigebracht \\
\hline 316 & und zwar hatte die ähm so verschiedene Reagenzgläser und dann da die Farbflüssigkeiten \\
\hline 317 & reingetan und dann haben die die in den Reagenzgläsern gemischt und alles in so einem \\
\hline 318 & Setting von so Laboranten () Wir untersuchen jetzt die Farbe und das war super \\
\hline 319 & hätte ich nie gedacht dass man da die Reagenzgläser aufstellt und dann mal die \\
\hline 320 & Flüssigkeiten mischt ne () also die Farbflüssigkeit blau mit gelb und guckt was passiert \\
\hline 321 & war total toll (.) ähh und ich ähh hätte die natürlich wieder mit dem Pinselset da mischen \\
\hline 322 & lassen \\
\hline 323 & Also so finde ich es wichtig den Austausch mit Kollegen und um damit es eben nicht so \\
\hline 324 & trocken wird auf Dauer mhh und ähm ja sich selber immer Informationsquellen neue holen \\
\hline & () auf dem Laufenden bleiben () was lesen () Internet () Lieder et cetera \\
\hline
\end{tabular}

Formulierende Interpretation

Im Gespräch geht dieser Episode eine Frage nach dem Zusammenhang von eigener Motivation und Verbesserungsmöglichkeiten im Unterricht voraus. Anne spricht daraufhin das Thema der eigenen professionellen Weiterentwicklung durch kollegialen Austausch und Mitwirkung von Praktikanten und Referendaren an. Sie nennt das Beispiel einer Unterrichtsstunde einer Kunst- und Chemiereferendarin, die sie Anne - in ihrem Fach Kunst betreut hatte. In einer Unterrichtsstunde in Kunst wurde ein für sie - Anne - völlig neuer Zugang zur Farbenlehre durchgeführt.

\section{Reflektierende Interpretation}

Nach einigen Äußerungen zu den Materialien initiiert Anne selbst das Thema des kollegialen Austauschs und der Mitwirkung von Praktikanten und Referendaren. Sie wechselt dabei von der relativ emotionslosen Beschreibung in die Erzählung und folgt dabei einem überraschenden Detaillierungszwang. Im Detail wird eine 
Episode aus dem Chemieunterricht erzählt. Anne beschreibt, dass Chemie für sie „immer () also völlig weit weg [war] () konnte ich nie mit was anfangen“. Die Referendarin überrascht sie mit einem völlig anderen Zugang zur Farbenlehre. Anne erzählt lebhaft, im Präsens, was in der Unterrichtsepisode passierte. Es entsteht das Bild eines sehr lebendigen Experiments, eine Unterrichtsmethode, die im Fremdsprachenunterricht unüblich ist. Anne zeigt sich begeistert von der völlig anderen Fachkultur („Und das war super“, „War total toll“) und elaboriert diese mit einer zusätzlichen Erläuterung („Hätte ich nie gedacht dass man da die Reagenzgläser aufstellt und dann mal die Flüssigkeiten mischt ne also die Farbflüssigkeit blau mit gelb und guckt was passiert"). Am Ende konkludiert sie das Thema im Modus einer Synthese: „Also so finde ich es wichtig den Austausch mit Kollegen und um damit es eben nicht so trocken wird auf Dauer mhh und ähm ja sich selber immer Informationsquellen neue holen () auf dem Laufenden bleiben () was lesen () Internet () Lieder et cetera". Das Leitmotiv ist hierbei die Weiterentwicklung des eigenen Unterrichts durch Information.

\subsubsection{Fallvergleich narrativer Interviews}

In diesem begrenzten Rahmen von lediglich zwei Fällen und vier bzw. drei Transkriptauszügen kann ein Fallvergleich nur rudimentär und die davon ausgehende Typenbildung nicht valide durchgeführt werden. Die folgenden Ausführungen haben somit eine hinweisende Funktion. Es soll das Prinzip des Vergleichens von Fällen und die dazu gehörige Textsorte illustriert werden.

Folgende Themen werden in diesen Auszügen von Bianca und Anne bearbeitet:

- Berufswahlmotiv

- Aktuelle Motivation

- Bedenken

- Theorie-Praxis-Verhältnis

Bianca hielt sich ursprünglich nach eigenen Angaben auf Grund ihrer Ungeduld für den Lehrberuf nicht geeignet. Die Wende bahnte sich zwar schon durch die positive Erfahrung mit dem Nachhilfeunterricht an, konnte jedoch erst vollzogen werden, als sie ihr Mutproblem löste und eine bewusste Entscheidung traf. Sie wurde für diese - für sie mutige - Entscheidung durch die große Freude belohnt, die sie seitdem in Ausbildung und Beruf erlebt.

Auch Anne berichtet von einer Entscheidung, allerdings nicht als positive Schlüsselentscheidung, sondern ex negativum bzw. in Opposition zu einem Sprachenlernen aus Interesse. Ihr ursprüngliches Interesse galt dem Spanischlernen im Land und der Begegnung mit dem Neuen, was bei Bianca entfiel, da diese 
zweisprachig aufgewachsen war. Anne entschied sich für den Lehrerberuf, obwohl ihr ursprüngliches Interesse in der Unterrichtspraxis unbefriedigt bleibt („Schulspanisch“). Sie braucht nun eine plausible Begründung für ihre Berufswahl und konstruiert dafür Verstandesargumente wie die Stärkung der romanischen Sprachen gegen das übermächtige Englisch und die Bedeutung Lateinamerikas. Damit dokumentiert sich Annes Berufswahl als eine rationale Entscheidung, nicht als Herzensentscheidung, anders als bei Bianca, die lediglich den Mut aufbringen musste, ja zu ihrem Gefühl zu sagen. Anne reibt sich an der Normalitätserwartung einer Kongruenz von fachlichem und pädagogischem Interesse bei angehenden Fremdsprachenlehrern, eine Normalitätserwartung, die sie nicht erfüllen kann. Für Bianca ergibt sich bezüglich dieser Normalitätserwartung keinerlei Konflikt.

Biancas aktuelle Motivation speist sich vor allem aus dem Erfolgserlebnis gelingenden Unterrichts. Sie erlebt, dass ihre Lehrmethodik funktioniert (Unterrichtsinhalte an die Welt der Lernenden anpassen, sie „verpacken“), und sie erlebt die damit verbundene Motivation der Lernenden („einfach ähm (.) sehr schön zu sehen wie die Kinder dann motiviert sind und dann auch wirklich ä:::h (2) aufblühen“).

Auch Anne motiviert das eigene Erfolgserlebnis („Ja und schön ist auch wenn sie sich dann schon nach ein paar Wochen äh tatsächlich schon ein bisschen in der Sprache unterhalten können"), doch tritt auch hier wieder die erwähnte Ambivalenz zu Tage: Das Unterrichten ist eben nicht nur reine Freude, sondern auch mit einer Geduldsprüfung verbunden („und das dauert halt ewig; da kann man das auch zehnmal vorsagen"). Bianca hatte ursprünglich von sich selbst angenommen, zu ungeduldig für den Lehrberuf zu sein, um in der Praxis festzustellen, dass dies gar nicht zutrifft, während Anne das Merkmalspaar GeduldUngeduld im Moment der Berufswahl gar nicht in Erwägung gezogen hatte und nun in der Berufspraxis feststellt, dass diese ihre Geduld manchmal über Gebühr beansprucht. Dennoch konkludiert Anne das Interview letztlich positiv mit dem Beispiel der Chemie-Referendarin. Das Beispiel kann auch als spezifische Facette von beruflicher Motivation gedeutet werden, insofern, als diese Art kollegialer Hospitation ihr neue Anregungen gibt.

Im Laufe des Interviews versucht der Interviewer mehrfach, Annes Ambivalenz weiter herauszufordern, und Anne geht auch teilweise explizit darauf ein. Am deutlichsten artikuliert sie ihre beruflichen Bedenken mit Blick auf die Korrekturen. Diese stellen für sie eine unangemessene zeitliche Belastung dar und symbolisieren überdies ein negatives Lehrkonzept, das sie nicht teilt. Sie würde ein Lehrkonzept bevorzugen, dass sich nicht auf die negative Fehlerkorrektur („mit dem roten Stift alles anzukreiden“) stützt. Noch grundsätzlicher allerdings 
sind Annes berufliche Bedenken angesichts ihrer Geringschätzung der Lernersprachen („Schulspanisch“) im Vergleich zur authentischen Sprachlernerfahrung im Land selbst. Anne stellt somit ihre berufliche Erfahrung gleich doppelt in Frage, einmal im Hinblick auf die mangelnde Authentizität der Zielsprache im Unterricht und zum zweiten im Hinblick auf das von ihr verlangte Lehrkonzept, das auf einer negativen Evaluationspraxis beruht. Hinzu kommt die Langsamkeit des Kompetenzaufbaus im Anfangsunterricht. Alle drei Aspekte sind elementare fachdidaktische Themen: das Verständnis der Struktur und des Funktionierens von Lernersprachen, das Verständnis der Funktion und Wirkung bestimmter Fehlerkonzepte und das Verständnis der Funktion und Wirkung von sprachlichem Input im Anfangsunterricht. Zu allen drei Themen orientiert sich Anne an einem negativen Gegenhorizont.

Derlei Bedenken sind Bianca fremd. Sie wendet grundsätzlich jegliche schulpraktischen Erfahrungen ins Positive, baut allerdings einen negativen Gegenhorizont hinsichtlich ihrer Studien an der Hochschule auf, die sie grundsätzlich als eher praxisfern bezeichnet („letztendlich ist aber die die Realität im Schulalltag ne ganz andere"). Auch die offiziellen Vorgaben und die Lehrbücher, die gemäß einer Normerwartung als eventuell hemmende Faktoren empfunden werden könnten, werden von Bianca als hilfreich für den Beruf dargestellt. Bianca orientiert sich damit an einem Professionsverständnis, das nur die Praxis mit all ihren Facetten bis hin zu den offiziellen Vorgaben wie Lehrpläne und Bildungsstandards als Basis für die eigene Entwicklung gelten lassen will. Gleichzeitig benutzt sie die didaktische Fachsprache und spricht von einem "guten Polster“ aus dem Studium.

Anne bezieht sich hingegen nicht explizit auf den Ertrag fachdidaktischer Theorie. Wir können nur indirekt aus ihrer dreifachen Negativdeutung bezüglich Lernersprachen, Evaluationskonzept und Anfangsunterricht schließen, dass sie die fachdidaktische Theorie nicht konstruktiv nutzen kann, um die praktischen Spannungsverhältnisse aufzulösen. Anne artikuliert sich zwar auf fachsprachlich bzw. fachdidaktisch anspruchsvollem Niveau, kann jedoch den Problemlösetransfer nicht leisten. Die Theorie bleibt bei ihr ein träges Wissen, während sie bei Bianca unbewusst möglicherweise zu praktischem Handlungswissen geworden ist, ohne dass Bianca allerdings bereit oder im Stande wäre, diese Deutung zuzulassen.

Berufsbiographisch könnte Annes „grundsätzlich anderer Ansatz“ irgendwann einmal zu einer Krise führen, die sie in eine „aufsteigende“ oder eine „absteigende Verlaufskurve“ (s. Kap. 2.4) führt. Bianca hingegen äußert an keiner Stelle auch nur einen Rest Zweifel an ihrer Berufswahl, sie lässt noch nicht 
einmal eine Spur von Bedenken diesbezüglich erkennen. Biancas Rahmung divergiert an dieser Stelle vollständig von Annes Rahmung, wenn auch beide einem affirmativ-positivierenden Habitus verpflichtet sind. Anne sieht die institutionellen Vorgaben (wie die weitere Analyse ergibt: Klassengrößen, Korrekturen, Notenvergabe) als Zwang, dem sie unterworfen ist und den sie in keiner Weise rechtfertigt. Sie vermeidet somit die Fremdrahmung. Ihr eigenes Lehrkonzept (Mündlichkeit, keine Noten, individuelle Förderung) weicht deutlich davon ab. Auffallend ist auch Annes Tendenz zu vergleichen: Sie vergleicht den Fremdsprachenunterricht mit Sport, Kunst und Chemie, sie vergleicht korrekturintensive Fächer mit vermeintlich weniger korrekturintensiven Fächern. Der Vergleich fällt für den Fremdsprachenunterricht häufig negativ aus, was darauf verweist, dass Anne mit ihrer Fächerwahl nicht voll zufrieden ist.

Die institutionellen Vorgaben sind Annes Projektionsfläche für die Ansätze der Unzufriedenheit, während Bianca den neuen beruflichen Rahmen für sich als vollkommen kongruent darstellt und Kritik ausschließlich auf die erste Phase der Lehrerbildung (Hochschule, Pädagogik und Didaktik) projiziert. Biancas Grundrahmung ist mithin die einer vollständigen professionsbezogenen Identifikation verbunden mit einer Sekundärrahmung durch die Negation hochschuldidaktischen Wissens und die parallele Absolutsetzung praxisgenerierten Handlungswissens („learning by doing“). Annes Grundrahmung hingegen ist die einer professionsbezogenen Ambivalenz verbunden mit einem permanenten Ausbalancieren divergenter Orientierungen (z.B. authentisches Spanisch versus Schulspanisch, Sprachenlernen im Zielland versus mühseliges Üben im Fremdsprachenunterricht, positives Feedback versus „Rotstift“).

Beide Interviews können als Anhaltspunkte für eine spätere Typenbildung zum Habitus von Fremdsprachenunterrichtenden als Berufsanfänger dienen (vgl. Blömecke \& König 2009; Herricks 2008; Herzog 2011). Im weiteren Verlauf der Studie könnten an Hand der Gesamttranskripte die ermittelten Orientierungen ergänzt und ihre innere Homogenität (s. Kap. 3.1.5) überprüft werden. Zur Ermittlung der äußeren Heterogenität (ibd.) müssten jedoch zunächst im Sinne der Kontrastbildung weitere Erhebungen durchgeführt werden: Interviews mit männlichen Berufsanfängern, mit Studierenden im Praktikum, mit Lehramtsanwärtern, mit Seiteneinsteigern, mit älteren Berufsanfängern und mit berufserfahrenen Unterrichtenden.

Die narrativen Interviews mit Bianca und Anne geben einen Eindruck von den Möglichkeiten, die die Dokumentarische Methode im Hinblick auf die Analyse der Verständigung über das Lehren von Fremdsprachen bietet. Es können z.B. generationen-, geschlechts- und bildungsmilieutypische Orientierungsmuster 
herausgearbeitet werden, sofern es gelingt, eine hinreichende Auswahl kontrastiver Fälle zu untersuchen. Aber auch ein einfacher Vergleich zweier Fälle mit relativ kurzer biografischer Verlaufsgeschichte wie bei Bianca und Anne ermöglicht, wie oben gezeigt, bereits Einblicke in die Auseinandersetzung von Junglehrerinnen mit professionellen Normalitätserwartungen.

Mit diesen beiden Fällen schließt die „Fallwerkstatt“. Im nun folgenden letzten Kapitel der Schrift gebe ich einen Ausblick auf mögliche weitere Forschungsfelder der rekonstruktiven Fremdsprachenforschung mit der Dokumentarischen Methode. 
Bernd Tesch - 978-3-631-69542-5

Downloaded from PubFactory at 01/11/2019 10:14:38AM

via free access 


\section{Ausblick}

Verschiedene Forschungen zum Fremdsprachenunterricht mit der Dokumentarischen Methode haben gezeigt, dass sie relevante Beiträge zum fachlichen Verstehen im bilingualen Sachfachunterricht Englisch (Bonnet 2004), zum Umgang mit komplexen Lernaufgaben im Französischunterricht (Tesch 2010) sowie zur literarischen Anschlusskommunikation im Englischunterricht (Bracker 2015) liefern kann. Sie scheint ein angemessener methodisch-methodologischer Referenzrahmen zu sein, um auch die Theorie des fremdsprachlichen Klassenzimmers (s. Breen 1985; Esteve 2010; Legutke 2006) insgesamt zu befruchten. Um diese Theorie und ihre Elemente etwas näher zu beschreiben, sei an dieser Stelle Legutke zitiert:

[...] gilt es, ausgehend von den bekannten Ansätzen der handlungs- und Erfahrungsorientierung das fremdsprachliche Klassenzimmer als eigene Kultur zu konfigurieren und die darin gestalteten Diskurse zu bestimmen. Dabei sind die dynamischen und wandelbaren Beziehungen ebenso zu berücksichtigen, die Lernende und Lehrende im Prozess des Sprachenlernens eingehen, wie die Themen und Texte, mit denen sich die Beteiligten auseinandersetzen und dabei gemeinsam die Lernumgebung und die Lerngemeinschaft gestalten. Entscheidungsspielräume und Aushandlungsprozesse werden dabei ebenso ins Blickfeld kommen müssen wie die unterschiedlichen Dimensionen kooperativen Lernens. Der Begriff des classroom discourse ist aus einer solchen Perspektive neu zu bestimmen und aus seiner eingeschränkten Bedeutung, die lediglich unterrichtsorganisatorische Handlungen bezeichnet, zu befreien. Die Theorie wird ferner angesichts der neuen Möglichkeiten, welche die digitalen Medien bereitstellen, den Handlungsraum aber auch die neuen Möglichkeiten multimedialer Textproduktion ausloten.

(Legutke 2006: 144)

Alle genannten Komponenten - Lehrende, Lernende, Themen, Texte, Medien, Interaktion und Diskurse - laden zu ihrer rekonstruktiven Erforschung mit der Dokumentarischen Methode ein. Eine sie alle umfassende Theorie fußt auf einem Verständnis des Fremdsprachenunterrichts als konjunktivem Erfahrungsraum, der institutionell eingerichtet ist, um zielorientiert und aufgabengeleitet Lernhandlungen zu initiieren. Diese Lernhandlungen generieren fachspezifische bzw. für den Fremdsprachenunterricht typische Wissensstrukturen, die als Lernersprache eine explizite bzw. direkt beobachtbare Ebene der Verständigung und als sozial geteilte Orientierungen eine implizite, aber rekonstruierbare Verständigungsebene darstellen.

Eine isolierte Untersuchung fachdidaktischer Forschungsgegenstände betrachte ich losgelöst von der sozialen Bedingtheit des fremdsprachlichen Klassenzimmers 
bzw. Unterrichts als problematisch. Stattdessen wäre eine Verbindung entsprechender empirischer Forschungen zu diesen Forschungsgegenständen mit Forschungen zur sozialen Interaktion im Unterricht aus meiner Sicht grundsätztlich vorteilhaft, um die für den Fremdsprachenunterricht charakteristische Relation von Sozialität und Individualität abzubilden.

Was aus sprach- oder literaturdidaktischer Sicht wünschenswert erscheint, wird in der Praxis des Unterrichtsalltags auf besondere Weise transformiert und erhält individuelle Ausprägungen. Unter Umständen werden theoretische Konzepte dabei sogar wesentlich verändert, wie sich etwa beim Konzept der Lernaufgabe im Fremdsprachenunterricht zeigte (Tesch 2010). Zur Erläuterung: Lernaufgaben beinhalten - als an reale Sprachverwendungssituationen angelehntes Unterrichtsarrangement - bestimmte intendierte Lernhandlungen. In der praktischen Umsetzung zeigen sich aber derart substantielle Anpassungen, dass diese nahelegen, sie als konzeptionellen Bestandteil einer Lernaufgabe von vornherein mitzudenken. Die individuelle Kreativität und Transformationsfähigkeit der Lernenden kommt damit voll und ganz zu ihrem Recht, befreit didaktische Großkonzepte von ihrer Unverbindlichkeit und eröffnet eine praxeologische Perspektive in der Fachdidaktik. Auch das Lehrerhandeln und die Transformationsleistungen der Lehrpersonen müssten in ihren Wechselwirkungen mit anderen didaktischen Konzepten zwingend mitberücksichtigt werden.

,Ergebnisse' des Fremdsprachenunterrichts werden in dieser Perspektive nicht mehr allein an einer bestimmten Normvorstellung oder an einem bestimmten intendierten Ziel gemessen. Vielmehr besteht nunmehr die Möglichkeit, rekonstruktiv auch das als Ergebnis mit in die Betrachtung des Unterrichts einzubeziehen, was aus normativer Sicht möglicherweise unbedeutend und unerwünscht erscheint: bestimmte implizite Wissensbestände der Lehrenden und Lernenden, das ,beiläufig' Gelernte, das Geflüsterte, die Randzeichnung auf dem Arbeitsblatt, das Nebengespräch, die Abweichung vom Thema, ja die ,Störung' des Unterrichts als affektive Mitwirkung an der Sinnkonstruktion. Hier liegt der besondere Erkenntnisgewinn der Dokumentarischen Methode im Verbund mit anderen Methoden der Fremdsprachenforschung. Sie erlaubt es, den Unterrichtsalltag und seine Praxis, die sich in sozialen Interaktionsmustern manifestieren, zu analysieren. Einen weiteren Mehrwert sehe ich in der Breite der Foki und Sinnbezüge, die durch die Dokumentarische Methode abgebildet werden können: Der Textsinn und der Aufgabensinn werden mit ihrer Hilfe ebenso einbezogen wie der Aufgabenbearbeitungssinn, sei es direkt im Zuge seines praktischen Vollzugs, sei es post hoc im Rahmen des Metagesprächs über den erlebten Unterricht oder der narrativen Rekonstruktion im Interview. 
Somit tritt neben den Schlüsselbegriff der Konstruktion (s. Kap. 1) derjenige der Transformation des Wissens. Fachdidaktisches Wissen in Form von Aufgaben wird in der Unterrichtspraxis in Lernendenwissen transformiert. Dieses Lernendenwissen und die vorausgehende Transformation sind sprachlich und inhaltlich sozial konstruiert. Wissenstransformation ist ein Wesensmerkmal des schulischen Lernens, und ohne diese Transformation zu verstehen sind Lernergebnisse nur schwer interpretierbar. Deshalb ist es bedeutsam, diese Erkenntnisse in der Lehrerbildung einzubeziehen. Wenn es gelingt, die Wissenstransformation und -konstruktion als Ergänzung zur Kompetenz- und Aufgabenorientierung in der Lehrerbildung zu implementieren, könnten m. E. einige Ursachen für das berechtigte Unbehagen vieler Unterrichtender an den abstrakten Großkonzepten ausgeräumt werden. Dazu jedoch wäre es erforderlich, auch den Methoden der rekonstruktiven empirischen Forschung stärker zu ihrem Recht zu verhelfen und die Lehrerbildung mit entsprechenden Materialien auszustatten, nämlich mit Lernprozessdokumenten in Form von Audio- und Videomitschnitten, Transkripten und Zwischenprodukten des Lernens, d.h. Schülertexten aller Art. Dies erfordert ein gewisses Umdenken, auch auf der administrativ-rechtlichen Ebene. Die Bereitstellung solcher Dokumente sollte datenschutzrechtlich nicht zu sehr behindert werden. Es erfordert aber zunächst einmal bei allen Akteuren der Lehrerbildung eine Bewusstwerdung für die Bedeutung dieser Dokumente und des mit ihnen verbundenen Lehrerbildungskonzepts.

Auf der Forschungsebene wiederum sind künftig weiterführende Anwendungen der Dokumentarischen Methode insbesondere zu folgenden Themenkomplexen wünschenswert:

- Lernersprache (Lernertexte) im Zusammenspiel individueller und sozialer Konstruktionsprozesse,

- das Zusammenspiel der Entwicklung von Lernersprache und sozialer Konstruktion fachlichen Wissens,

- generell das Verhältnis von Individualität und Sozialität beim fremdsprachlichen Lehren und Lernen,

- sprachliche und inhaltliche Aushandlungsprozesse in zunehmend multikulturell-mehrsprachigen und inklusiven Klassen,

- der Einfluss neuer medialer Lernumgebungen auf die Interaktion im fremdsprachlichen Klassenzimmer,

- die doppelte Transformation der Aufgabe auf den unterschiedlichen Lernniveaus und an unterschiedlichen Lerngegenständen: von der task as a workplan zur task as a process (Ellis 2003) und von der task as a process zur Aufgabe als Wissensstruktur Lernender, 
- die Kategorisierung ,typischer ' Unterrichtsarrangements und eine darauf bezogene rekonstruktive Lehr-Lern-Typologie,

- eine allgemeine Typologisierung der Aufgabenbearbeitung im Fremdsprachenunterricht,

- eine Metastudie zu den bisher vorliegenden rekonstruktiven Studien mit der Dokumentarischen Methode.

Eine große methodisch-methodologische Herausforderung weiterer rekonstruktiver Unterrichtsforschungen mit der Dokumentarischen Methode liegt in der adäquaten typologischen Modellierung der individuellen Lernersprachen und der individuellen Sprachbewustheit im Zusammenspiel mit den sozialen Typiken der Aufgabenbearbeitung. Insbesondere im Bereich der weiteren Erforschung der Lernersprachen liegt es nahe, methodische Triangulationen anzustreben (z.B. mit den Verfahren des Lauten Denkens: Ericsson \& Simon 1980, und des Lauten Erinnerns: z.B. Gass \& Mackey 2000). Sollte es gelingen, Ressourcen und Forschungsinteressen hierhin zu lenken, dürfte es möglich sein, der Antwort auf die Frage „Was ist Fremdsprachenlehren und -lernen“ ein großes Stück näher zu kommen. 


\section{Bibliographie}

Allwright, Richard (1984): The importance of interaction in classroom language learning. Applied Linguistics 5,2, 156-171.

Altrichter, Herbert \& Posch, Peter ( $\left.{ }^{3} 1998\right)$ : Lehrer erforschen ihren Unterricht: Eine Einführung in die Methoden der Aktionsforschung. Bad Heilbrunn: Klinkhardt.

Asbrand, Barbara (2011)/Dokumentarische Methode/ http://www.fallarchiv. uni-kassel.de/wp-content/uploads/2010/07/asbrand_dokumentarische_me thode.pdf. [15.05.2016]

Asbrand, Barbara (2009): Wissen und Handeln in der Weltgesellschaft: Eine qualitativ-rekonstruktive Studie zum globalen Lernen in der Schule und in der außerschulischen Jugendarbeit. Münster: Waxmann.

Ballis, Anja; Penzold, Michael; Scherf, Daniel \& Schieferdecker, Ralf (2014): Die Dokumentarische Methode und ihr Potenzial für Forschungen (nicht nur) in der Fachdidaktik Deutsch. In: Didaktik Deutsch 37, 2014, 92-104.

Bandura, Albert (1997): Self-efficacy: The exercise of control. New York: Freeman.

Batstone, Rob \& Philp, Jenefer (2013): Classroom interaction and learning opportunities across time and space. In: McDonough, Kim \& Mackey, Alison (Hg.): Second Language Interaction in Diverse Educational Contexts. Amsterdam/Philadelphia: John Benjamins Publishing Company, 109-125.

Blömecke, Sigrid \& König, Johannes (2009): Pädagogisches Wissen von angehenden Lehrkräften. Erfassung und Struktur von Ergebnissen der fächerübergreifenden Lehrerausbildung. In: Zeitschrift für Erziehungswissenschaft $12,499-527$.

Börner, Wolfgang (2000): Interaktion in Lernaufgaben. In: Bausch, Karl-Richard u. a. (Hg.): Interaktion im Kontext des Lehrens und Lernens fremder Sprachen: Arbeitspapiere der 20. Frühjahrskonferenz zur Erforschung des Fremdsprachenunterrichts. Tübingen: Narr, 45-50.

Bohnsack, Ralf ( $\left.{ }^{2} 2014\right)$ : Rekonstruktive Sozialforschung. Einführung in qualitative Methoden. Stuttgart: UTB.

Bohnsack, Ralf \& Przyborski, Aglaja (2006): Diskursorganisation, Gesprächsanalyse und die Methode der Gruppendiskussion. In: Bohnsack, Ralf, Przyborski, Aglaja \& Schäffer, Burkhard (Hg.): Das Gruppendiskussionsverfahren in der Forschungspraxis. Opladen: Leske und Budrich, 233-248.

Bohnsack, Ralf; Nentwig-Gesemann, Iris \& Nohl, Arndt-Michael (Hg.) (2007): Die Dokumentarische Methode und ihre Forschungspraxis. Grundlagen qualitativer Sozialforschung. Wiesbaden: VS-Verlag. 
Bonnet, Andreas (2004): Chemie im bilingualen Unterricht. Kompetenzerwerb durch Interaktion. Opladen: Leske und Budrich.

Bonnet, Andreas (2009): Die Dokumentarische Methode in der Unterrichtsforschung: ein integratives Forschungsinstrument für Strukturrekonstruktion und Kompetenzanalyse. In: Zeitschrift für Qualitative Forschung 10, 2, 219-240. URN: http://nbn-resolving.de/urn:nbn:de:0168-ssoar-339871. [15.05.2016]

Bonnet, Andreas (2012): Von der Rekonstruktion zur Integration:Wissenssoziologie und Dokumentarische Methode in der Fremdsprachenforschung. In: Doff, Sabine (Hg.): Fremdsprachenunterricht empirisch erforschen. Grundlagen Methoden - Anwendung. Narr. Tübingen: Narr, 286-305.

Bourdieu, Pierre (1976): Entwurf einer Theorie der Praxis. Frankfurt a. M.: Suhrkamp (Im Original: Esquisse d'une théorie de la pratique précédé de trois études d'ethnologie kabyle. Genève: Droz, 1972).

Bourdieu, Pierre (1982): Die feinen Unterschiede - Kritik der gesellschaftlichen Urteilskraft. Frankfurt a. M.: Suhrkamp (Im Original: La distinction. Critique sociale du jugement. Paris 1979).

Bracker, Elisabeth (2012): Die Dokumentarische Methode als Instrument zur Analyse von literarischer Anschlusskommunikation. In: Doff, Sabine (Hg.): Fremdsprachenunterricht empirisch erforschen. Grundlagen - Methoden - Anwendung. Tübingen: Narr, 306-315.

Bracker, Elisabeth (2015): Fremdsprachliche Literaturdidaktik: Plädoyer für die Realisierung bildender Erfahrungsräume im Unterricht. Wiesbaden: Springer.

Bredella, Lothar \& Christ, Herbert (1995): Didaktik des Fremdverstehens im Rahmen einer Theorie des Lehrens und Lernens fremder Sprachen. In: Dies.: (Hg.), Didaktik des Fremdverstehens. Tübingen: Narr, 8-19.

Bredella, Lothar (2007): Bildung als Interaktion zwischen literarischen Texten und Leser/innen. Zur Begründung der rezeptionsästhetischen Literaturdidaktik. In: Bredella, Lothar \& Hallet, Wolfgang (Hg.): Literaturunterricht, Kompetenzen und Bildung. Trier : WVT, 39-68.

Breen, Michael P. (1985): Authenticity in the language classroom. Applied Linguistics 6, 1, 60-70.

Bremerich-Vos, Albert; Granzer, Dietlinde \& Köller, Olaf (2008): Gute Aufgaben für den Unterricht. Weinheim: Beltz.

Bruner, Jerome S. (1985): Narrative and paradigmatic modes of thougt. In: Elliot Eisner (Hg.): Learning and teaching the ways of knowing. Chicago, IL: University of Chicago Press, 97-115.

Bundesministerium für Bildung und Forschung (Hg.) (2003): Zur Entwicklung nationaler Bildungsstandards: Eine Expertise. Berlin. 
Burwitz-Melzer, Eva (2007): Ein Lesekompetenzmodell für den fremdsprachlichen Literaturunterricht. In: Bredella, Lothar \& Hallet, Wolfgang (Hg.): Literaturunterricht, Kompetenzen und Bildung. Trier: WVT, 127-158.

Burwitz-Melzer, Eva \& Caspari, Daniela (2016): Lernaufgaben: Definitionen, Prinzipien und Kriterien, In: Tesch, Bernd; von Hammerstein, Xenia; Stanat, Petra \& Rossa, Henning (Hg.): Bildungsstandards aktuell: Englisch/Französisch in der Sekundarstufe II. Braunschweig: Diesterweg, 244-265.

Caspari, Daniela (2003): Fremdsprachenlehrerinnen und Fremdsprachenlehrer: Studien zu ihrem beruflichen Selbstverständnis. Tübingen: Narr.

Cazden, Courtney (1988): Classroom Discourse: The language of teaching and learning. Portsmouth NH: Heinemann.

Chirbes, Rafael (2013): En la orilla. Barcelona: Anagrama.

Decke-Cornill, Helene (2013): Ästhetische Erfahrung im Schülergespräch über einen Kurzfilm. In: Grünewald, Andreas; Plikat, Jochen \& Wieland, Katharina (Hg.): Bildung - Kompetenz - Literalität. Fremdsprachenunterricht zwischen Standardisierung und Bildungsanspruch. Seelze: Klett-Kallmeyer, 163-178.

Eberle, Thomas S. (1984): Sinnkonstitution in Alltag und Wissenschaft. Der Beitrag der Phänomenologie und die Methodologie der Sozialwissenschaft. Bern: Haupt.

Eckerth, Johannes (2003): Fremdsprachenerwerb in aufgabenbasierten Interaktionen. Tübingen: Narr.

Eichler, Wolfgang \& Nold, Günter (2007): Sprachbewusstheit. In: Beck, Bärbel \& Klieme, Eckard (Hg.): Sprachliche Kompetenzen. Konzepte und Messung. DESI-Studie (Deutsch Englisch Schülerleistungen International). Weinheim: Beltz, 63-82.

Ellis, Rod (2003): Task based teaching and learning. Oxford: Oxford University Press.

Ericsson, Karl A. \& Simon, Herbert A. (1980): Verbal Reports as Data. In: Psychological Review, 87, 215-251.

Esteve Ruescas, Olga (2006): Interacción, conciencia linguística y desarrollo de la autonomía en el apendizaje de lenguas extranjeras. In: Monográficos marco ELE 10, 69-82.URL: http://marcoele.com/descargas/expolingua_2006.esteve. pdf. [05.02.2016].

Europarat. Rat für kulturelle Zusammenarbeit (Hg.) (2001): Gemeinsamer europäischer Referenzrahmen für Sprachen: Lernen, Lehren, Beurteilen. Berlin München/Wien/Zürich/New York: Langenscheidt. (Abgekürzt GeR)

Finkbeiner, Claudia (2013): Lernstrategien und Lerntechniken im Kontext neuer Unterrichtsaufgaben. In Bach, Gerhard \& Timm, Johannes-Peter (Hg.): Englischunterricht, Francke: Tübingen, Basel. (5. Auflage). 
Finkbeiner, Claudia \& Knierim, Markus (2008): Aufgabenorientiertes Lernen im Englischunterricht: Beispiele zur Förderung kognitiver, metakognitiver und sozial-affektiver Lernprozesse. In: Thonhauser, Josef (Hg.): Aufgaben als Katalysatoren von Lernprozessen. Münster: Waxmann, 149-167.

Flick, Uwe (2003): Triangulation. In : Bohnsack, Ralf ; Marotzki, Winfried \& Meuser, Michael (Hg.) : Hauptbegriffe qualitativer Sozialforschung. Opladen: Budrich.

Funke, Peter (1990): Das Verstehen einer fremden Kultur als Kommunikationsprozess. In: Die Neueren Sprachen 89, 6, 584-596.

Garfinkel, Harold \& Sacks, Harvey (1976): Über formale Strukturen praktischer Handlungen. In: Sack, Fritz \& Schenkein, Jim (Hg): Ethnomethodologie - Beiträge zu einer Soziologie des Alltagshandelns. Fft. a. M., 130-176.

Gass, Susan M. \& Mackey, Alison (2000): Stimulated Recall Methodology in Second Language Research. Mahwah, New Jersey: Lawrence Erlbaum Associates.

Genette, Gérard (1998): Die Erzählung. Paderborn: Fink.

Glaser, Barney G. \& Strauss, Anselm L. (1967): The Discovery of Grounded Theory. Strategies for Qualitative Research. New York: de Gruyter.

Glinka, Hans-Jürgen (2008): Das narrative Interview in seinen zentralen Analyseschritten. Tübingen: VS-Verlag.

Gnutzmann, Claus (1997): Language Awareness. Geschichte, Grundlagen, Anwendung. In: Praxis des neusprachlichen Unterrichts 44, 3, 227-236.

Goffman, Erving (1981): Forms of Talk. Philadelphia: UPN.

Grzesik, Jürgen (1990): Textverstehen lehren und lernen. Geistige Operationen im Prozess des Textverstehens und typische Methoden für die Schulung zum kompetenten Leser. Stuttgart: Klett.

Gurwitsch, Arin (1979): Die mitmenschlichen Begegnungen in der Milieuwelt. Berlin/New York: de Gruyter.

Hallet, Wolfgang \& Nünning, Ansgar (Hg.) (2007): Neue Ansätze und Konzepte der Literatur- und Kulturdidaktik. Trier: Wissenschaftlicher Verlag Trier.

Harden, Theo (2006): Angewandte Linguistik und Fremdsprachendidaktik. Tübingen: Narr.

Helmke, Andreas (2002): Kommentar: Unterrichtsqualität und Unterrichtsklima. In: Unterrichtswissenschaft 30, 3, 261-277.

Herricks, Uwe (2008): Professionalisierung als Entwicklungaufgabe. Rekonstruktionen zur Berufseingangsphase von Lehrerinnen und Lehrern. Wiesbaden: VS Verlag für Sozialwissenschaften.

Herzog, Silvio (2011): Über den Berufseinstieg hinaus: Berufsbiografien von Lehrerinnen und Lehrern im Blickfeld der Forschung. In: Terhart, Ewald; 
Bennewitz, Hedda \& Rothland, Martin (Hg.): Handbuch der Forschung zum Lehrerberuf. Münster: Waxmann, 315-338.

Imdahl, Max (1994): Ikonik. Bilder und ihre Anschauung. In: Boehm, Gottfied (Hg.): Was ist ein Bild? München: Fink, 300-324.

Imdahl, Max (1996): Giotto - Arenafresken. Ikonographie - Ikonologie - Ikonik. München: Fink.

Iser, Wolfgang (1972): Der implizite Leser. München: Fink.

Jauß, Hans Robert (1970): Literaturgeschichte als Provokation der Literaturwissenschaft. Frankfurt a. M.: Suhrkamp.

James, Carl \& Garrett, Peter (Hg.) (1991): Language awareness in the classroom. London: Longman.

Kallenbach, Christiane (1996): Subjektive Theorien: Was Schüler und Schülerinnen über Fremdsprachenlernen denken. Tübingen: Narr.

Kallmeyer, Werner \& Schütze, Fritz (1976): Konversationsanalyse. In: Studium der Linguistik, 1, 1-28.

Kallmeyer, Werner \& Schütze, Fritz (1977): Zur Konstitution von Kommunikationsschemata der Sachverhaltsdarstellung. Dargestellt am Beispiel von Erzählungen und Beschreibungen. In: Wegner, Dirk (Hg.): Gesprächsanalysen. Hamburg: Goldmann, 159-274.

Kern, Richard (2000): Literacy and Language Teaching. Oxford: Oxford University Press.

Keutel, Klaus \& Grossarth, Jan (2014): Der Kompetenz-Fetisch. Frankfurter Allgemeine Zeitung 14.-15.02.2015, S. C3.

Klafki, Wolfgang (2006): Die bildungstheoretische Didaktik im Rahmen kritischkonstruktiver Erziehungswissenschaft. In: Gudjons, Herbert \& Winkel, Rainer (Hg.): Didaktische Theorien. Hamburg: Bergmann und Helbig Verlag, 13-34.

Kleemann, Frank; Krähnke, Uwe \& Matuschek, Ingo (2009): Interpretative Sozialforschung. Eine praxisnahe Einführung. Wiesbaden: VS-Verlag.

Köller, Olaf; Knigge, Michel \& Tesch, Bernd (Hg.) (2010): Sprachliche Kompetenzen im Ländervergleich. Überprüfung der Erreichung der Bildungsstandards für den Mittleren Schulabschluss für Deutsch und die erste Fremdsprache in der neunten Jahrgangsstufe. Münster: Waxmann.

Lantolf, James P. \& Poehner, Matthew E. (2008): Sociocultural Theory and the Teaching of Second Languages. London: Equinox.

Legutke, Michael K. (2006): Aufgabe - Projekt - Szenario: Über die großen Perspektiven und die kleinen Schritte. In: Bausch, Karl-Richard et al. (Hg.): Aufgabenorientierung als Aufgabe: Arbeitspapiere der 26. Frühjahrskonferenz zur Erforschung des Fremdsprachenunterrichts. Tübingen: Narr, 140-148. 
Levelt, Willem J. M. (1989): Speaking: From intention to articulation. Cambridge: MIT Press.

Long, Michael (1996): The role of the linguistic environment in second language acquisition. In: Ritchie, William \& Bhatia, Tej (Hg.): Handbook of second language acquisition. San Diego: Academic Press, 413-468.

Lucius-Hoene, Gabriele \& Deppermann, Arnulf (2004): Rekonstruktion narrativer Identität. Ein Arbeitsbuch zur Analyse narrativer Interviews. Wiesbaden: VS-Verlag.

Mackey, Alison (1999): Input, Interaction and Second Language Development. In: Studies in Second Language Acquisition 21, 557-587.

Mangold, Werner \& Bohnsack, Ralf (1988): Kollektive Orientierungen in Gruppen Jugendlicher. Bericht für die Deutsche Forschungsgemeinschaft. Erlangen: Manuskript.

Mannheim, Karl (1980): Strukturen des Denkens. Frankfurt a. M. (Original 19221925, unveröff. Manuskripte).

Mead, George Herbert (1934): Mind, Self, and Society. Edited by Charles W. Morris. Chicago. (Deutsch: Geist, Identität und Gesellschaft aus der Sicht des Sozialbehaviorismus. Fft a. M.: Suhrkamp, 1968.)

Meißner, Franz-Joseph \& Tesch, Bernd (Hg.) (2010): Spanisch kompetenzorientiert unterrichten. Grundlagen und Beispiele zur Aufgabenkonstruktion. Stuttgart: Klett.

Michel, Burkhard (2010): Das Gruppendiskussionsverfahren in der (Bild-) Rezeptionsforschung. In: Bohnsack, Ralf; Przyborski, Aglaja \& Schäffer, Burkhard (Hg.): Das Gruppendiskussionsverfahren in der Forschungspraxis, Opladen: Budrich, 219-232.

Morton, Janne; Storch, Neomy \& Thompson, Celia (2014): Feedback on student writing in the supervision of postgraduate students: Insights from the work of $\mathrm{Vy}$ gotsky and Bakhtin. In: Journal of Academic Language and Learning, 8, 1, 24-36.

Müller-Hartmann, Andreas \& Schocker-von Dithfurth, Marita (2011): Teaching English: Task supported Language Teaching. Paderborn: Schöningh.

Nentwig-Gesemann, Iris (2007): Die Typenbildung der Dokumentarischen Methode. In: Bohnsack, Ralf; Nentwig-Gesemann, Iris \& Nohl, Arndt-Michael (Hg.): Die Dokumentarische Methode und ihre Forschungspraxis. Grundlagen qualitativer Sozialforschung. Wiesbaden: VS-Verlag, 277-302.

Nohl, Arnd-Michael (2005): Dokumentarische Interpretation narrativer Interviews. In: Bildungsforschung, 2, 2, URL: http://www.bildungsforschung.org/ Archiv/2005-02/interview/ [17.05.2016]

Nohl, Arnd-Michael ( $\left.{ }^{4} 2012\right)$ : Interview und Dokumentarische Methode - Anleitungen für die Forschungspraxis. Wiesbaden: VS-Verlag. 
Nohl, Arnd-Michael (2013): Relationale Typenbildung und Mehrebenenvergleich. Zur Untersuchung gesellschaftlicher Heterogenität. Wiesbaden: Springer.

Nünning, Ansgar (2007): Fremdverstehen und Bildung durch neue Weltansichten: Perspektivenvielfalt, Perspektivenwechsel und Perspektivenübernahme durch Literatur. In: Hallet, Wolfgang \& Nünning, Ansgar (Hg.): Neue Ansätze und Konzepte der Literatur- und Kulturdidaktik. Trier: Wissenschaftlicher Verlag Trier, 123-142.

Oevermann, Ulrich (1979): Sozialisationstheorie - Ansätze zu einer soziologischen Sozialisationstheorie und ihre Konsequenzen für die allgemeinsoziologische Analyse. In: Kölner Zeitschrift für Soziologie und Sozialpsychologie, Sonderheft 21, 143-168.

Oevermann, Ulrich (1991): Genetischer Strukturalismus und das Problem der Entstehung des Neuen. In: Müller-Doohm, Stefan (Hg.): Jenseits der Utopie. Fft a. M.: Suhrkamp, 267-338.

Panofsky, Erwin (1975): Ikonografie und Ikonologie. Eine Einführung in die Kunst der Renaissance. In: Ders.: Sinn und Deutung in der bildenden Kunst. Köln: DuMont, 36-67.

Philp, Jenefer; Adams, Rebecca \& Iwashita, Noriko (2014): Peer Interaction and Second Language Learning. New York, London: Routledge.

Przyborski, Aglaja (2004): Gesprächsanalyse und Dokumentarische Methode. Qualitative Auswertung von Gesprächen, Gruppendiskussionen und anderen Diskursen. Wiesbaden: Springer.

Przyborski, Aglaja \& Wohlrab-Sahr, Monika (2009): Qualitative Sozialforschung. Ein Arbeitsbuch. München: Oldenbourg.

Ransom, John Crowe (1941): The New Criticism. Norfolk, Conn: New Directions.

Sacks, Harvey; Schegloff, Emanuel A. \& Jefferson, Gail (1974): A Simplest Systematics for the Organization of Turn Taking for Conversation. In: Language, 50, 696-735.

Schütz, Alfred (1974): Der sinnhafte Aufbau der sozialen Welt. Eine Einleitung in die Verstehende Soziologie. Frankfurt a. M.: Suhrkamp (Wien: Springer 1932).

Schütze, Fritz (1976): Zur Hervorlockung und Analyse von Erzählungen thematisch relevanter Geschichten im Rahmen soziologischer Feldforschung - dargestellt an einem Projekt zur Erforschung von kommunalen Machtstrukturen. In: Arbeitsgruppe Bielefelder Soziologen: Kommunikative Sozialforschung. München: Fink, 159-260.

Schütze, Fritz (1983): Biografieforschung und narratives Interview. In: Neue Praxis. Kritische Zeitschrift für Sozialarbeit und Sozialpädagogik, 13, 3, 283-293.

Sekretariat der Ständigen Konferenz der Kultusminister der Länder in der Bundesrepublik Deutschland (Hg.) (2014): Bildungsstandards für die fortgeführte 
Fremdsprache (Englisch/Französisch) für die Allgemeine Hochschulreife. Köln: Wolters Kluwer.

Selinker, Larry (1972): Interlanguage. In: International Review of Applied Linguistics 10, 3, 31-54.

Selting, Margret et al. (2009): Gesprächsforschung - Online-Zeitschrift zur verbalen Interaktion 10, 353-402.

Steininger, Ivo (2014): Modellierung literarischer Kompetenz. Tübingen: Narr Francke Attempto.

Storch, Neomy (2013): Collaborative language learning, in Chapelle Carol A. (Hg.): The Encyclopedia of Applied Linguistics. Hoboken: Wiley-Blackwell Publishing, 725-730.

Strauss, Anselm \& Corbin, Juliet ( $\left.{ }^{2} 1996\right)$ : Grundlagen Qualitativer Sozialforschung. Weinheim: Beltz.

Strohn, Meike (2015): Binnendifferenzierung im Englischunterricht. Bochum: Projekt-Verlag.

Tesch, Bernd (2010): Kompetenzorientierte Lernaufgaben im Fremdsprachenunterricht. Konzeptionelle Grundlagen und eine rekonstruktive Fallstudie zur Unterrichtspraxis (Französisch). Fft a. M.: Peter Lang.

Tesch, Bernd (2014): Warum sollen wir das lernen? Überlegungen zur Beteiligung von Lernenden an der Auswahl von Lerngegenständen im Fremdsprachenunterricht. In: Zeitschrift für die romanischen Sprachen und ihre Didaktik, $8,2,49-69$.

Tesch, Bernd (2015): Vielfalt: Sprachendidaktische Annäherungen an einen gesellschaftlichen Leitbegriff am Beispiel politischer Bilder. In: Zeitschrift für die romanischen Sprachen und ihre Didaktik, 10, 1, 35-67.

Tesch, Bernd; von Hammerstein, Xenia; Stanat, Petra \& Rossa, Henning (2016, im Druck): Bildungsstandards aktuell: Englisch/Französisch in der Sekundarstufe II. Braunschweig: Diesterweg.

The New London Group (2000): A pedagogy of multiliteracies. Designing social futures. In: Cope, Bill \& Kalantzis, Mary (Hg.): Multiliteracies. Literacy learning and the design of social futures. London, New York: Routledge, 9-37.

Thonhäuser, Josef (Hg.) (2008): Aufgaben als Katalysatoren von Lernprozessen. Eine zentrale Komponente organisierten Lehrens und Lernens aus der Sicht von Lernforschung, Allgemeiner Didaktik und Fachdidaktik. Münster: Waxmann.

Trautmann, Matthias (2014): Fremde Sprachen und Fremdsprachenlernen aus Schülersicht: eine Interviewstudie zu fremdsprachlichen Identitätskonstruktionen von Oberstufenschülerinnen. Opladen: Budrich.

Ulm, Volker; Cwik, Gabriele \& Metzger, Klaus M. et al. (Hg.) (2008): Gute Aufgaben Mathematik. Berlin: Cornelsen. 
Van Lier, Leo (1984): Analysing interaction in second language classrooms. In: ELT Journal 38, 3, 160-169.

Van Lier, Leo (2004): The ecology and semiotics of language learning: A sociocultural perspective. Norwell, MA: Kluwer.

Vollmer, Helmut Johannes (2016): Sprachbewusstheit. In: Tesch, Bernd et al. (Hg.): Bildungsstandards aktuell: Englisch/Französisch in der Sekundarstufe II. Braunschweig: Diesterweg, 201-219.

Vygotsky, Lev ('1971): Denken und Sprechen. (Russische Originalausgabe 1934, deutschsprachige Ausgabe Akademie-Verlag, Berlin 1964, gekürzt). Fft. a. M.: Fischer.

Wäckerle, Maike \& Martinez, Hélène (2016): Umsetzung und Weiterentwicklung von Lernaufgaben. In: Tesch, Bernd et al. (Hg.): Bildungsstandards aktuell: Englisch/Französisch in der Sekundarstufe II. Braunschweig: Diesterweg, 266-286.

Wellek, René (1978): The New Criticism: Pro and Contra. In: Critical Inquiry, 4, 4, 611-624.

Wertsch, James V. (2007): Mediation. In: Daniels Harry \& Wertsch, James V. (Hg.): The Cambridge Companion to Vygotsky. Cambridge: Cambridge University Press, 178-192.

Wimsatt, William K. \& Beardsley, Monroe C. (1954): The Verbal Icon: Studies in the Meaning of Poetry. Lexington: University of Kentucky Press.

Winkels, Gianna (2015): Die literarische Anschlusskommunikation bei der Arbeit mit einem Comic im Spanischunterricht. Eine rekonstruktive Fallstudie mit der Dokumentarischen Methode. Unveröffentlichte Wissenschaftliche Hausarbeit. Universität Kassel.

Wolff, Dieter (2002): Fremdsprachenlernen als Konstruktion: Grundlagen für eine konstruktivistische Fremdsprachendidaktik. Frankfurt a. M.: Peter Lang.

Wood, David; Bruner, Jerome S. \& Ross, Gail (1976): The role of tutoring in problem solving. In: Journal of Child Psychology \& Psychiatry \& Allied Disciplines, 17, 2, 89-100.

Zeitler, Sigrid; Heller, Nina \& Asbrand, Barbara (2012): Bildungsstandards in der Schule. Eine rekonstruktive Studie zur Implementation der Bildungsstandards. Münster: Waxmann.

\section{Zitierte Lehrwerke}

¡Apúntate! Cornelsen 2008.

Adelante intermedio. Klett 2011. 
Bernd Tesch - 978-3-631-69542-5

Downloaded from PubFactory at 01/11/2019 10:14:38AM

via free access 


\section{Abbildungs- und Tabellenverzeichnis}

Abb. 1 Entwicklungszonen des lernersprachlichen Sinns

Abb. 2 LE MONDE, 16. November 2013, cérémonie daccueil

Abb. 3 LE MONDE, 16. November 2013, cérémonie d'accueil, Fluchtachsen

Abb. 4 Eugène Delacroix, 1830, La Liberté guidant le peuple, Fluchtachsen

Abb. 5 Arbeitsblatt der Lernaufgabe Pir@tes du net

Abb. 6 Textauszug aus ;Apúntate!

Abb. 7 Arbeitsblatt zu iApúntate!

Abb. 8 Titelbild des Comics Ne me quitte pas

Abb. 9 Ausschnitt aus Arbeitsblatt 0 der Unterrichtssequenz Ne me quitte pas

Abb. 10 Arbeitsblatt I der Unterrichtssequenz zu Ne me quitte pas

Abb. 11 Orientierungen bei der Aufgabenbearbeitung des Comics Ne me quitte pas

Abb. 12 Sinngenetische Typik bei der Aufgabenbearbeitung des Comics Ne me quitte pas

Tab. 1 Sinnebenen des Fremdsprachenlehrens und -lernens

Tab. 2 Rahmungen und Orientierungen im Fremdsprachenunterricht

Tab. 3 Listenartige thematische Gliederung einer Gruppendiskussion im Anschluss an ein fachdidaktisches Seminar

Tab. 4 Modi der Diskursorganisation

Tab. 5 Lernersprachliche turns und soziale Diskursorganisation

Tab. 6 Strukturebenen für die Klassifizierung von Fällen

Tab. 7 Interpretationsraster für biografische Kurzinterviews

Tab. 8 Argumentstruktur des Comics Ne me quitte pas

Tab. 9 Thematische Grobgliederung Gruppe I, Aufgabenbearbeitung I bis III

Tab. 10 Thematische Gliederung einer Themenaushandlung im Unterricht

Tab. 11 Narratives Interview „Bianca“, thematische Gliederung

Tab. 12 Narratives Interview „Anne“, thematische Gliederung 
Bernd Tesch - 978-3-631-69542-5

Downloaded from PubFactory at 01/11/2019 10:14:38AM

via free access 


\section{Glossar}

Dieses Glossar zur rekonstruktiven Fremdsprachenforschung mit der Dokumentarischen Methode fußt auf dem Glossar in Tesch 2010 (397 ff.); dieses wurde grundlegend überarbeitet und ergänzt. Querverweise sind durch $(\rightarrow)$ gekennzeichnet.

\section{Antithese}

Reaktion auf eine Proposition im Sinne eines Widerspruchs. Die antithetisch formulierte Aussage kann sich anders als die oppositionelle in einem gemeinsamen $(\rightarrow)$ Orientierungsrahmen mit der Aussage bewegen, auf die sie reagiert. Dieser gemeinsame Orientierungsrahmen kann im weiteren Diskursverlauf deutlich werden bzw. am Ende verworfen werden.

\section{Atheoretisches Wissen}

Un- oder vorbewusstes Wissen von Gesprächspartnern, das ein intuitives unmittelbares Verstehen ermöglicht. Dieses Wissen ist nach Mannheim (1980) prinzipiell strukturidentisch mit dem Wissen der Forschenden, das heißt die Produktionsregeln der Verständigung sind prinzipiell die gleichen.

\section{Aufgabensinn}

Die von Aufgabenentwicklern intendierten und in die Aufgabenstellung(en) eingebetteten Lern- und Arbeitsziele. Der Aufgabensinn (intendierte Sinn) kann erheblich vom $(\rightarrow$ ) realisierten Sinn in der Aufgabenbearbeitung abweichen.

\section{Authentizität}

Evidenz von $(\rightarrow)$ Orientierungsmustern bzw. ihrer Darstellung; abhängig von ihrer erlebnismäßigen Verankerung (Erfahrungsgebundenheit).

\section{Basisregeln der Kommunikation}

Allgemeine Prinzipien und Aktivitäten der Kooperation nach Kallmeyer \& Schütze $(1976,1977)$. Sie gelten auch für den schulischen Unterricht und beinhalten die Bedingungen der Möglichkeit von Kommunikation. Kern dieser Bedingungen ist die Bewältigung grundsätzlicher Unvereinbarkeiten des Interaktionsprozesses durch Kooperativität, Verstehen/Verständlichkeit und Ablaufkonstitution. 


\section{Biografische Gesamtformung}

Eine in sich geschlossene Gestalt (Persönlichkeitstypus nach Bohnsack), die beim biografischen Interview bereits vorliegt. Durch fallinternen Vergleich wird dieser herausgearbeitet, bevor der Vergleich mit anderen Fällen es erlaubt, Typen etwa auf der Ebene einer Geschlechts- oder Generationentypik - zu bestimmen.

\section{Differenzierung}

Weiter- bzw. Ausarbeitung einer $(\rightarrow)$ Proposition, ohne dass daraus ein Gegenhorizont gebildet wird.

\section{Diskursentwicklung}

In der Diskurslinguistik: mehrstufige Abfolge der Diskursschritte Orientierung

- Ausführung - Kontrolle; in der Wissenssoziologie: Proposition - Elaboration - Konklusion.

\section{Dokumentsinn, dokumentarischer Sinn}

Ziel der $(\rightarrow)$ reflektierenden Interpretation ist es, den Dokumentsinn zu rekonstruieren und dadurch den $(\rightarrow)$ Habitus sichtbar werden zu lassen.

\section{Dramaturgie}

Bezogen auf die Diskursentwicklung: In den dramatischen Höhepunkten kommen kollektive $(\rightarrow)$ Orientierungen in besonders prägnanter Weise zum Ausdruck. Die Dramaturgie lässt sich anhand der systematischen Rekonstruktion formaler Indikatoren des Gesprächsverlaufs (Übereinstimmungen im Bereich von Code, Prosodie, Tempo, Pausen, Sprecherwechsel) ermitteln.

\section{Einklammerung des Geltungsanspruchs}

Vom $(\rightarrow)$ immanenten Sinn abgegrenzte Bedeutungen zur Aussage/zum Text/ zum Bild. Es wird abgegrenzt, was eine Aussage außerhalb ihres immanenten Sinns bedeuten kann, könnte oder bedeuten soll. Damit wird auch der Anspruch auf normative Richtigkeit „eingeklammert“ (s. Mannheim 1980: 88).

\section{Elaboration}

Ausbau bzw. Ausarbeitung einer Proposition, z.B. durch Beispiele und Vergleiche.

\section{Enaktierung}

Prozess der Umsetzung von $(\rightarrow)$ Orientierungen in Alltagshandeln. 


\section{Exkludierende Modi}

Innerhalb der $(\rightarrow)$ Diskursentwicklung: oppositionelle, antithetische und divergente Modi aufeinanderfolgender Äußerungen. Diese Modi weisen auf eine Rahmeninkongruenz hin (Ausnahme: Antithese).

\section{Exmanenter Sinn}

Die den Partnern einer Verständigung i. d. R. verborgene, weil auf unbewussten sozialen Konstruktionen beruhende Sinnebene. Sie kann nur dadurch erschlossen werden, dass der Forscher die Relevanzsysteme der Beforschten verlässt und sich ihnen gegenüber „fremd macht“.

\section{Externe Heterogenität}

Abweichungen von Orientierungsmustern bezogen auf $(\rightarrow)$ Rahmenorientierungen, die zur Abgrenzung eines Typus gegenüber anderen Typen bzw. Typiken benötigt werden. Durch $(\rightarrow)$ maximale Kontrastierung und die Suche nach der maximalen Unterschiedlichkeit im Fallvergleich können diese Abweichungen zusammen mit der $(\rightarrow)$ minimalen Kontrastierung - ermittelt werden.

\section{Fallbeschreibung}

Beschreibung des - den besonderen Fall konstituierenden - Rahmens oder seiner Rahmungen. Die Beschreibung folgt der prozesshaften Entwicklung des Diskursverlaufs. Der Forschende fügt die verschiedenen Analyseergebnisse zu einem kohärenten Text zusammen, der sie für Außenstehende nachvollziehbar werden lässt. Ansatzweise können der Fallbeschreibung bereits Typiken zugeordnet werden.

\section{Formulierende Interpretation}

Zusammenfassung des Transkripttextes in Form von Oberbegriffen, Überschriften und Paraphrasen zu den Themen, die angesprochen wurden; dieser Analyseschritt bleibt innerhalb des Orientierungsrahmens der Gruppe, innerhalb des $(\rightarrow)$ immanenten Sinngehalts.

\section{Fokussierungsmetapher}

Dramaturgischer Höhepunkt in der Diskursentwicklung, häufig im Modus metaphorischer Formulierungen; zentriert die $(\rightarrow)$ reflektierende Interpretation durch Zugang zur $(\rightarrow)$ Authentizität. 


\section{Fremdverstehen}

Das (vermeintliche) Verstehen der $(\rightarrow)$ Relevanzsysteme Anderer durch ihre Adaptation an das eigene Relevanzsystem. Basiskonzept rekonstruktiver Sozialforschung nach Schütz (1974). Methodisch kontrolliertes Fremdverstehen wird durch kommunikative Validierung in der Forschergruppe angestrebt. In der Didaktik des Fremdverstehens von Bredella \& Christ (1995) Leitbegriff für das Verstehen fremdsprachiger Texte.

\section{Gegenhorizont}

Von Einzelnen formulierte positive oder negative Aussage, die den $(\rightarrow)$ Rahmen identifizierbar macht bzw. aus Rahmenorientierungen abgeleitet ist. Aus der Rekonstruktion der Gegenhorizonte lässt sich auf die Rahmenorientierungen schließen.

\section{Grounded Theory}

Von Glaser und Strauss (1967) begründetes Forschungsparadigma, das auf standardisierte Messung und Hypothesenprüfung verzichtet und stattdessen die Theoriebildung in den Forschungsprozess selbst verlagert. Der Forschende sucht solange „im Feld“ nach Fällen, bis die Theorie „gesättigt“ ist, d.h. keine neuen Aspekte mehr zu Tage treten.

\section{Habitus}

Die Art und Weise eines sozialen Auftretens. Im Fremdsprachenunterricht sind Beispiele für einen Habitus das positivierende Verhalten und die entsprechende professionelle Einstellung eines Junglehrers oder das gewohnsheitsmäßige Kategorisieren von Lernenden nach ihren Leistungen. Nach Bourdieu (1982) strukturiert der Habitus sowohl das Handeln als auch die Wahrnehmung und Bewertung eigenen und fremden Handelns.

\section{Homologie}

Gleichgelagertsein von Fällen; gilt als Voraussetzung eines methodisch begründeten Vergleichs.

\section{Homologieannahme}

Annahme, dass das Wissen von Forschern und Beforschten strukturidentisch ist, was wiederum eine Voraussetzung für die Rekonstruktion dieses Wissens durch Forschende darstellt. Auch Schütze (1976) spricht von der Homologieannahme 
mit Bezug auf das narrative Interview: In der Struktur der Erzählung spiegelt sich das Erleben der Person.

\section{Immanenter Sinn}

Wörtlicher Sinngehalt, auch als kommunikativer Sinn bezeichnet. Gemeint ist der Sinngehalt, der von den Teilnehmern einer Verständigung selbst wahrgenommen und verhandelt wird. Er kann durch die $(\rightarrow)$ formulierende Interpretation erschlossen werden.

\section{Indexikalität}

Verweischarakter kommunikativer Handlungen in der ethnomethodologischen Forschung. Kommunikative Handlungen verweisen auf zugrunde liegende Orientierungsmuster, die durch den Forschenden rekonstruiert werden können.

\section{Inkludierende Modi}

Innerhalb der $(\rightarrow)$ Diskursentwicklung: paralleler und univoker Modus aufeinanderfolgender Äußerungen.

\section{Interne Homogenität}

Abweichungen innerhalb eines bestimmten Typus, die durch minimale Kontrastierung innerhalb eines Falles bestimmt werden können; sie wird zur genauen Beschreibung des Typischen und zur Abgrenzung gegenüber anderen Typiken verwendet.

\section{Komparative Analyse}

Auf die $(\rightarrow)$ Grounded Theory (Glaser \& Strauss 1967) zurückgehende Methode des ständigen Vergleichens von Fällen bis zur „theoretischen Sättigung“. Ihre Anwendung als Analyseprinzip führt auf der sinngenetischen Ebene zur Bildung von Basiskategorien, die in der Dokumentarischen Methode generell auch als "Typus" bezeichnet werden.

\section{Konjunktiver Erfahrungsraum}

Raum sozial geteilter Praxis, der die Erfahrungsgrundlage für die Konstruktion sozial geteilten Wissens bildet. Eine Schulklasse teilt nicht nur das Klassenzimmer als (physischen) Erlebnisraum, sondern vor allem die gemeinsame soziale Praxis des alltäglichen Sinnaushandelns. Die gemeinsame soziale Praxis konstituiert das Klassenzimmer als konjunktiven Erfahrungsraum. 


\section{Konklusion}

Ende einer Diskursentwicklung und damit auch eines Themas, entweder in Form einer sachlichen Zusammenfassung, eines rituellen Abschlusses oder einer Metakommunikation.

\section{Lernersprache}

Begriff aus der Linguistik (Interlanguage, Selinker 1971) für die individuellen fremdsprachlichen Entwicklungsstände zwischen Ausgangssprache und Zielsprache. Diese Entwicklungsstände sind regelhaft, dynamisch und veränderbar. Performatorisch können sie als individuell beschrieben, genetisch jedoch auch als kollektive Konstruktion betrachtet werden. Während der urprünglich linguistische Begriff der Interlanguage auf den interimären Entwicklungsstand des individuellen Sprachsystems fokussierte, bezieht der fachdidaktische Begriff der Lernersprache auch das lernende Subjekt gleichberechtigt mit ein.

\section{Lernersprachlicher Sinn}

Sprachlicher Sinn, der sich aus dem bewussten und unbewussten Sprach(en)wissen von Fremdsprachenlernenden generiert. Dieser Sinn kann sich performativ äußern, z.B. in der Kenntnis bestimmter fremdsprachiger Lexik, wie auch in Form von $(\rightarrow)$ Sprachbewusstheit bezogen auf sprachliche Regeln und Systeme, auf Register und Varietäten sowie sprachliche Beeinflussung.

\section{Opposition}

Reaktion auf eine Proposition im Sinne eines Widerspruchs. Es kann jedoch im Gegensatz zur Antithese - kein gemeinsamer Orientierungsrahmen gefunden werden.

\section{Orientierungsmuster}

$(\rightarrow)$ Rahmen/Rahmung

\section{Praxeologische Fremdsprachenforschung}

Untersucht, was in der Praxis des Fremdsprachenunterrichts und allgemein des Fremdsprachengebrauchs geschieht und wie es geschieht. In der Fremdsprachenforschung wird Praxis als kollektive Sinnkonstruktion in der Verständigung durch Texte, über Texte und Themen und über das Lehren und Lernen von Sprachen verstanden. Daher können Praxen des Fremdsprachenlehrens und -lernens unterschieden werden. 


\section{Proposition}

Eine Aussage als Ausgangspunkt in einem Diskursverlauf. Ihr semantischer Gehalt beziehungsweise Orientierungsgehalt kann in der Interpretation durch den Forscher rekonstruiert werden. Auf die Proposition folgt eine Reaktion, entweder in Form einer Anschlussproposition oder in Form eines Widerspruchs (als Antithese oder Opposition).

\section{Rahmen / Rahmung}

Ordnungskategorie für unbewusste Orientierungsmuster des Einzelnen oder einer Gruppe. Der Rahmen manifestiert sich in den Themen, die Einzelne in einem Interview oder Gruppen in einem Gruppengespräch anschneiden oder bearbeiten. Wird der Orientierungsrahmen in einer Gruppe nicht geteilt, kann es zu Aufspaltungen und Rahmeninkongruenzen kommen. Inkongruenzen können offen sichtbar oder verdeckt sein. Fremdrahmung entsteht, wenn die Äußerungen eines Teilnehmers von einem anderen Gruppenmitglied übernommen bzw. in dessen Rahmen transponiert werden, mit der Folge, dass dessen eigene Rahmen eliminiert wird.

\section{Realisierter Sinn}

Ergebnis einer Aufgabenbearbeitung im Sinne rekonstruktiver Forschung. Der realisierte Sinn beinhaltet eine kommunikative $(\rightarrow)$, eine dokumentarische $(\rightarrow)$ und eine lernersprachliche $(\rightarrow)$ Dimension und kann erheblich vom intendierten Sinn einer Aufgabe(nbearbeitung) abweichen.

\section{Reflektierende Interpretation}

Rekonstruktion und Explikation eines $(\rightarrow)$ Rahmens durch Herausarbeiten der $(\rightarrow)$ Gegenhorizonte, die - zusammen mit den $(\rightarrow)$ Enaktierungspotenzialen den Erfahrungsraum einer Gruppe bilden. Dabei werden Orientierungsmuster fallübergreifend oder fallintern verglichen, d.h. entweder in ähnlichen Thematiken in verschiedenen Fällen oder in verschiedene Thematiken im selben Fall.

\section{Relevanzsystem}

Typische Orientierungsmuster beforschter Personen, die mit Hilfe der $(\rightarrow)$ reflektierenden Interpretation rekonstruierbar gemacht werden können.

\section{Sampling}

Fallauswahl. 


\section{Scaffolding}

Die Bereitstellung von Lernhilfen in Anlehnung an die Lerntheorie Vygotskys $(\rightarrow$ Zone of Proximal Development). Wörtlich: Gerüstebauen.

\section{Sinnebenen des Fremsprachenunterrichts}

Die Ebene des $(\rightarrow)$ Aufgabensinns (auch: intendierter Sinn), die Ebene des $(\rightarrow)$ kommunikativen Sinns, die Ebene des $(\rightarrow)$ dokumentarischen Sinns und die Ebene des $(\rightarrow)$ lernersprachlichen Sinns. Letztere interagiert transversal mit den drei anderen Sinnebenen.

\section{Sinngenetische Typenbildung}

Erster Schritt der $(\rightarrow)$ Typenbildung: Aus dem Vergleich empirisch ermittelter Orientierungsmuster resultiert deren Abgrenzung und Bestimmung.

\section{Sinnkonstitution}

Die Sozialität und Strukuriertheit von Phänomenen bzw. Sinngebilden. Bezug auf die Phänomenologie nach Schütz (1974).

\section{Sinnkonstruktion}

Das kollektive Hervorbringen von Sinn in der Verständigung. Bezug auf die Wissenssoziologie Mannheims (1980).

\section{Soziogenetische Typenbildung}

Zweiter Schritt der Typenbildung: Nach der $(\rightarrow)$ sinngenetischen Typenbildung erfolgt die Suche nach individualpsychologischen und sozialpsychologischen Erklärungen.

\section{Sprachbewusstheit}

Explizites und implizites Wissens bezüglich der Regelhaftigkeit, des Gebrauchs und der Wirkung von Sprache. James \& Garrett (1991) unterscheiden fünf Domänen von Sprachbewusstheit, eine affektive, eine soziale, eine politische, eine performatorische und eine kognitive Domäne.

\section{Sprachlernbiografie}

Die Erfassung der Voraussetzungen und des individuellen Werdegangs, die zum Erwerb sprachlichen Wissens führen. Die Sprachlernbiografie umfasst institutionelle Voraussetzungen (z.B. gegebene Landes- und Verkehrssprachen, Herkunftssprachen, Schullaufbahn und Sprachenwahl), psycho-physische Voraussetzungen (Alter, Geschlecht, körperliche Verfassung, Intelligenz etc.), 
soziales und kulturelles Kapital (Milieu, Elternhaus, Bildungsstatus etc.) sowie Motivation.

\section{Tertium comparationis}

Das zu vergleichende Dritte in der rekonstruktiven Sozial- und Fremdsprachenforschung.

\section{Turn}

In der Diskurslinguistik die kleinste Diskurseinheit: Ein Sprecher ergreift das Wort, reagiert ggf. auf eine vorangegangene Äußerung und macht selbst eine Äußerung, auf die dann andere wieder reagieren können.

\section{Typus, Typik, Typenbildung}

Herstellung von Bezügen „zwischen spezifischen Orientierungen und dem Erlebnishintergrund oder existentiellen Hintergrund, in dem die Genese der Orientierungen zu suchen ist" (Bohnsack 2003: 141). Beispiele für Typenbildungen sind die Milieu-, Geschlechts- und Generationstypik. Eine Typik ist umso valider, je deutlicher sie zu anderen Typiken abgegrenzt werden kann. Nur das ständige Vergleichen ermöglicht die Abgrenzung von Typen untereinander.

\section{Validierung}

Bestätigung einer Proposition im Diskurs. Die schwache Form ist die Ratifizierung: bestätigt wird lediglich das Verstehen. Unter kommunikativer Valdierung versteht man die nachträgliche Validierung einer Interpretation durch Beforschte oder aber die Valdierung von Interpretationen durch eine Forschergruppe durch Diskussion.

\section{Zone of Proximal Development (ZPD)}

Naheliegende Entwicklungszone von Lernenden; Begriff von Vygotsky (1971), in dem das lernpsychologische Modell einer stufenweisen Entwicklung des Lernens zum Ausdruck kommt. Die nächste Lernstufe, die innerhalb der zone of proximal development liegt, kann ohne Überforderung erreicht werden, sofern Lehrer, Mitschüler oder geeignete Lernmaterialien Lernhilfen (scaffolds) anbieten. 
Bernd Tesch - 978-3-631-69542-5

Downloaded from PubFactory at 01/11/2019 10:14:38AM

via free access 


\section{Schlagwortverzeichnis}

A

Ablaufkonstitution 49, 177

Aktionsforschung 23, 165

aktuelle Entwicklungszone 69

Alltagshandeln 19, 178

Analyseeinstellung 14, 23, 54, 55, 57,

$61,120,121,126,130,136,149$

Angebot-Nutzungs-Modell 14

Anonymisierung 82

Anschlusskommunikation 21, 23,

$108,113,120,135,161,166,173$

Anschlussproposition 31, 183

Antithese 89, 90, 177, 182, 183

antithetisch $30,177,179$

atheoretisch 16, 18, 19, 33, 71, 81, 107

Atheoretisch 16, 177

Audiografie 15

Aufgabe 13, 18, 19, 28, 31, 47, 61, 62, $64,66,67,69,70,71,87,108,113$,

$115,118,119,121,122,130,131$,

$132,163,169,183$

Aufgabenbearbeitung 13, 14, 19, 20, $26,30,31,32,62,64,65,68,69,71$,

$107,118,120,121,122,123,124$, $127,128,129,130,132,133,134$,

$135,136,164,175,183$

Aufgabenbearbeitungsforschung 14, 21

Aufgabenforschung 14

Aufgabenkonzept 35, 63, 123

Aufgabennutzungsforschung 14

Aufgabenpotential 62,71

Aufgabensinn 19,61, 62, 63, 67, 113,

$115,116,119,120,162,177$

Aufgabenwirkungsforschung 14

aushandeln (von Bedeutung) 31

Authentizität 97, 115, 157, 177, 179

Autonomie 34, 88

autopoietisch 54
B

Basisregeln 48, 177

Bildkomposition 57, 58, 60, 61, 65

Bildpotential 63

Bimodale Texte 108

Biografische Gesamtformung 74, 75, 178

Biografischen Erzählung 73, 139

Biographische Handlungsmuster 73

Biographischer Wandlungsprozess 74

C

Classroom discourse 161

Code switching 68

Common ground 19, 42

Constant comparative method 97

D

Detaillierungszwang 75, 152, 154

Didaktik des Fremdverstehens 26, 50, 166,180

Differenzierung 8, 89, 90, 178

Diskursanalyse $31,71,77,91,105$

Diskursbewegung 21, 22, 68, 89, 90, 91

Diskursentwicklung 7, 25, 27, 46, 89, $178,179,181,182$

dokumentarischer Sinn 19, 88, 178

Dokumentarischer Sinn 19

Dokumentsinn 178

Dramaturgie 59, 178

E

Einklammerung des Geltungsbereichs 55

Elaboration 89, 90, 129, 142, 153, 178

Embodied language 80

emergent 127

Enaktierung 178, 183 
Episode $31,49,65,69,85,95,124,125$, $126,128,129,130,155$

Erfahrungsraum $17,44,45,54,75,76$, $77,92,100,131,133,137,161,181$, 183

Ergebnis 69, 70, 88, 98, 132, 162, 183

Erlebniskohärenz 48, 49

Erzähltheorie 50

Ethnomethodologie 43, 168

Exkludierende Modi 179

Exmanenter Sinn 179

expliziter Sinn 65, 119, 137, 156, 157

externe Heterogenität 98, 135

F

Fallbeschreibung 79, 92, 94, 95, 96, $101,105,131,133,179$

Fallvergleich 42, 96, 105, 131, 133, $134,155,179$

Fiktionalität 54

Fokussierungsmetapher 128, 146, 150,179

formulierende Interpretation 79,86 ,

$87,88,105,123,126,181$

Fremdheitsrelation 26

fremdkulturell 51, 54

Fremdkulturell 53

Fremdsprachenerwerb 17, 151, 167

fremdsprachliches Klassenzimmer 161

G

Ganzheitsstruktur 55, 57, 58, 61

Gegenhorizont 33, 43, 89, 90, 95, 96, $127,128,141,146,147,157,178,180$

Gemeinsamer europäischer Referenzrahmen für Sprachen (GeR) 167

Generationstypik 44, 76, 185

Geschlechtertypik 106

Gestaltschließungszwang 74

Grounded Theory 43, 97, 168, 180, 181
Gruppendiskussion 42, 51, 73, 75, 79, $86,87,104,133,165,175$

Gruppengesprächsanalyse $72,75,76$, $79,101,102,105$

Gütekriterien 98

$\mathbf{H}$

habitualisiert 16,43

Habitus 54, 55, 57, 70, 100, 153, 158, 178,180

Hermeneutik 13, 47, 104

Hermeneutik (objektive) 101

Heterogenität 158, 171, 179

Hinterbühne 31

homolog 77

Homologie 41, 180

Homologieannahme 73, 102, 180

I

Ikonik 54, 169

Ikonografie 54, 171

immanenter Sinn 50, 52, 178

Indexikalität $25,32,33,54,88,89$, 110,181

Individualität $7,40,46,48,70,75,135$, 162,163

inkludierende Modi 181

innerer Bilder 55, 57

institutionelles Ablaufmuster 73

intendierter Sinn 19, 32, 63, 67, 88, 184

Interaktion $7,14,19,20,21,27,31,51$, $64,65,72,80,85,86,92,100,104$, $124,146,161,162,163,165,166,172$

Interaktionismus (symbolischer) 16 interaktive Dichte 123, 128 interkomprehensiv 119 Interlanguage 17, 172, 182 interne Homogenität 181 intersubjektiv überprüfbar 26 
K

kommunikatives Wissen 18,111

kommunikativer Sinn 19, 88, 181

kommunikative Validierung 26, 80, 180

Komparative Analyse 181

Kompetenzorientierung 13, 23, 47

Kondensierungszwang 74

konjunktiver Erfahrungsraum 77

Konjunktiver Erfahrungsraum 181

konjunktiver Sinn 19

Konjunktivität 45, 46, 133

Konklusion 21, 22, 69, 89, 90, 91, 121, $127,128,147,178,182$

Konstruktion 12, 15, 17, 22, 34, 35, 41, $42,62,91,106,125,131,163,173$, 181,182

Kontextwissen 56

Kontrast 96, 102, 150, 152

Konversationsanalyse 13, 169

Kooperativität 49, 177

kulturelles Kapital 44, 185

L

Lautdenk-Protokoll 53

Lautes Denken 164

Lautes Erinnern 164

Lebenslauf 73, 77, 104

Lernaufgabe $63,93,96,97,100,107$, $115,121,123,133,136,162,175$

Lernersprache $17,18,24,25,32,34$, $40,41,50,65,68,69,70,71,72,77$, $79,91,93,124,132,135,153,161$, 163,182

Lerngruppenmilieu 25, 43

Lese-Gespräche 53

Literacies 50, 172

M

Maximaler Kontrast 97, 98, 179

Mediation 21, 65, 70, 173

Mehrebenenanalyse 94
Metapher 128, 141, 144, 151

metaphorische Dichte 85

Milieu 42, 43, 77

milieutypisches Lernverhalten 44

minimaler Kontrast 98, 181

Model-fit 98

modell 116

Modell 25, 40, 116, 185

multimodaler Text 79

Mutterspracherwerb 17

N

Narrationsanalyse 13

narratives Interview 8, 76, 102, 171

Narrativik 54, 92

Narratologisch 54

New Criticism 50, 167, 173

Normalität 104, 105, 138, 150, 156,159

Normalitätserwartung 147, 156

Normalitätsvorstellung 105

O

Opposition 89, 90, 155, 182, 183

Orientierungsmuster $16,69,101,136$, $159,181,182,183,184$

Orientierungsrahmen 45, 89, 94, 99, $177,182,183$

$\mathbf{P}$

paralleler Modus 181

Peers 13, 21, 31, 34, 35, 42, 65, 70, 96, 104

performativ 18, 19, 41, 62, 65, 182

Persönlichkeitstypus 75, 178

Perspektivendifferenzierung 50

Perspektivenwahrnehmung 50

Perspektivenwechsel 50, 51, 53,

54,171

Perspektivik 58,60

perspektivische Projektion 57

Phänomenologie $12,167,184$ 
phänomenologisch 16

planimetrisch 57, 58, 110

Poststrukturalismus 55

potenzielle Entwicklungszone 17

praktische Idealisierungen 48

Praxen 13, 23

praxeologisch 13, 23, 24, 41, 47, 62, $107,135,162$

Praxeologisch 14, 182

Praxis 13, 14, 15, 17, 23, 43, 64, 86, 87, $91,102,107,138,147,155,156,157$, $162,166,168,171,181,182$

Proposition 21, 22, 89, 90, 91, 121, $126,129,130,151,177,178,182$, 183,185

Prozessstruktur 73, 74, 75, 104

$\mathbf{R}$

Rahmen 94

Rahmenschaltelemente 103

Rahmung 19, 31, 34, 43, 65, 69, 90, 95, $123,133,135,144,158,182,183$

Ratifizierung 89, 90, 128, 185

Realisierter Sinn 183

Referenzrahmen 11,12, 13, 161, 167 reflektierende Interpretation 79,84 , $85,101,104,105,138,179$

Rekonstruktion 8, 15, 24, 25, 32, 33, $47,61,62,73,81,89,106,162,166$, $170,178,180,183$

Relevanz 34, 49, 65, 74, 85, 115, 147, 153

Relevanzfestlegunszwang 74

Relevanzsystem 32, 87, 121, 180, 183

Reliabilität 98

Rezeptionsästhetik 50

rezeptionsästhetisch 51,52, 166

Ritualität 80

S

sampling $76,93,135,183$

Scaffolding 121, 184
Selbstmonitoring 62,68 selbstreferentielles System 54

Selbstwirksamkeit 34, 46, 96, 131, 133, 134

Sinnebenen $8,13,18,19,24,40,88$, $91,112,175,184$

Sinngehalt 7, 16, 179, 181 sinngenetisch $89,92,97,101,181,184$ Sinngenetisch 134, 184

Sinnkonstitution $12,167,184$

Sinnkonstruktion $12,15,20,24,25$, $40,41,47,50,51,97,128,135,162$,

182,184

soziale Mediation 65

Sozialisationstheorie 47,171

Sozialität 7, 40, 43, 46, 47, 48, 70, 108, $135,162,163,184$

soziogenetische Typenbildung 184 soziokulturelle Theorie 17, 50, 91

Sprachbewusstheit $7,18,19,41,42$, $65,71,90,92,100,127,128,129,130$ $132,135,136,167,173,182,184$

Sprachenbiografie 70,71,73

Spracherwerb 17, 41

Sprachlernbiografie 92, 100, 184

Sprachübung 19, 30, 31, 32, 33, 34, 64, 132

Stegreiferzählung 73, 76

Subjektive Theorien 72, 169

symbolisch 16,111,114

szenische Choreografie 57, 60

$\mathrm{T}$

Task as a process 163

Task as a workplan 113, 163

Task-based learning 34

Teilnehmende Beobachtung 79, 80, 81

Tertiärsprachenerwerb 17

Tertium comparationis $69,89,91$, 93, 96

Textpotential $62,66,71,113,114$

Textsinn 50,61, 107, 110, 162 
Transformation $20,61,71,121$, 136,163

Transkript $83,96,103,121,128,132$

Transkription $16,79,82,83,84,85$, 102

Triangulation $80,81,106,168$

Turn 15, 27, 83, 91, 175, 185

Typenbildung 7, 25, 41, 44, 73, 76, 77, 79, 92, 96, 99, 100, 101, 131, 133, 134, $136,155,158,170,171$, 184,185

Typik 42, 44, 70, 76, 97, 99, 105, 134, $136,175,185$

Typologie $76,96,99,101,135,164$

Typus $42,43,44,97,98,101,135,179$, 181,185

$\mathrm{U}$

Um-zu-Motiv 100

univoker Modus 129, 181

V

Validierung 89, 90, 99, 109, 129, 185

Validität 25, 82, 88, 98, 99, 109

Verlaufskurve $73,74,157$
Vermittlung 21, 63, 92

Verständigung 7, 13, 14, 16, 18, 19, 20, $21,22,24,25,32,33,41,42,43,50$, $51,53,54,55,61,65,70,71,72,75$, $76,77,81,92,96,102,104,106,107$, $108,121,136,137,158,161,177$, $179,181,182,184$

Verstehen $26,33,41,49,50,53,65$, $71,109,115,136,153,161,168,177$, 180,185

Verweischarakter 33, 181

Videoaufzeichnungen 80

vortheoretisches Wissen 16

W

Wandlungsprozess $73,74,76$

Weil-Motiv 100

Wissenssoziologie $12,16,33,77,166$, 178,184

$\mathbf{Z}$

Zone der naheliegenden Entwicklung 17

Zone of Proximal Development (ZPD) $91,184,185$ 